\title{
Accessing Aliphatic Amines in C-C Cross-Couplings by Visible Light/Nickel Dual Catalysis
}

\author{
Weizhe Dong, Shorouk O. Badir, Xuange Zhang and Gary A. Molander[a] \\ Department of Chemistry, University of Pennsylvania \\ *gmolandr@sas.upenn.edu
}

1. General Considerations 2

$\begin{array}{ll}\text { 2. Synthesis of } \alpha \text {-Silylamines } & 4\end{array}$

3. Extended Optimizations and Control Studies 16

4. General Procedure for Aminomethylation of Aryl Halides 18

5. Procedure for Large Scale Reaction 43

6. Streamlined Synthesis of a Dipeptide Derivative $\quad 45$

$\begin{array}{ll}\text { 7. Mechanistic Studies } & 48\end{array}$

8. Cyclic Voltammogram of Representative Amines 55

$\begin{array}{ll}\text { 9. Crystallographic Data } & 60\end{array}$

$\begin{array}{ll}\text { 10. References } & 68\end{array}$

11. Spectra of Synthesized Compounds $\quad 69$ 


\section{General Considerations}

\subsection{General}

All chemical transformations requiring inert atmospheric conditions or vacuum distillation utilized Schlenk line techniques with a 4- or 5-port dual-bank manifold. LED irradiation was accomplished using the LED reactor described in our previous report ${ }^{[1]}$ or via a setup of two Kessil H150-Blue LED lamps (456 nm, 30 W High Luminous DEX 2100 LEDs) that were each placed 1.5 inches away from reaction vessels with two fans to ensure the reactions remained at room temperature (rt). Unless otherwise noted, benchtop photo reactions were performed in $4 \mathrm{~mL}$ Chemglass vials (1-dram, 15 x $45 \mathrm{~mm}$, 13-425 Green Open Top Cap, TFE Septa, part number: CG-4909-04) or 8 mL Chemglass vials (2-dram, 17 x 60 mm, 15-425 Green Open Top Cap, TFE Septa, part number: CG-4909-03). NMR Spectra $\left({ }^{1} \mathrm{H},{ }^{13} \mathrm{C}\left\{{ }^{1} \mathrm{H}\right\},{ }^{19} \mathrm{~F}\left\{{ }^{1} \mathrm{H}\right\}\right.$, $\left.{ }^{29} \mathrm{Si}\left\{{ }^{1} \mathrm{H}\right\}\right)$ were performed at $300 \mathrm{~K} .{ }^{1} \mathrm{H}$ NMR spectra were referenced to residual non-deuterated chloroform $\left(\delta\right.$ 7.26) in $\mathrm{CDCl}_{3}$, residual DMSO- $d_{5}(\delta 2.50 \mathrm{ppm})$ in DMSO- $d_{6} .{ }^{13} \mathrm{C}$ NMR spectra were referenced to $\mathrm{CDCl}_{3}(\delta 77.2 \mathrm{ppm})$ and DMSO- $d_{6}(\delta 39.5 \mathrm{ppm})$. Data is presented as follows: chemical shift $(\mathrm{ppm})$, multiplicity $(\mathrm{s}=$ singlet, $\mathrm{d}=$ doublet, $\mathrm{t}=$ triplet, $\mathrm{q}=$ quartet, $\mathrm{m}=$ multiplet, $\mathrm{br}=$ broad), coupling constant $J(\mathrm{~Hz})$, and integration. Reactions were monitored by LC/MS, GC/MS, ${ }^{1} \mathrm{H}$ NMR, and/or TLC using silica gel F254 plates (60 A porosity, $250 \mu \mathrm{m}$ thickness). TLC analysis was performed using EtOAc/hexanes or $\mathrm{CH}_{2} \mathrm{Cl}_{2} / \mathrm{MeOH}$ as the eluent and visualized using permanganate stain, ninhydrin stain, phosphomolybdic acid stain and/or UV light in $254 \mathrm{~nm}$ and/or $365 \mathrm{~nm}$ wavelengths. Flash chromatography was accomplished using automated flash chromatography system [with a UV detector monitoring at $254 \mathrm{~nm}, 280 \mathrm{~nm}$, and 200-300nm and an evaporative light scattering detector (ELSD)] with RediSep $^{\circledR}$ Rf silica gel disposable flash columns (60 A porosity, 40-60 $\mu \mathrm{m}$ ) or RediSep Rf Gold ${ }^{\circledR}$ silica

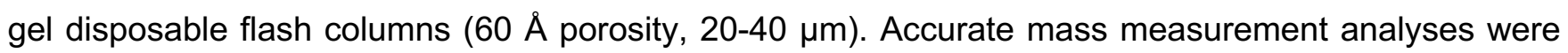
conducted using electron ionization (EI) or electrospray ionization (ESI). The signals were mass measured (TOF) against an internal lock mass reference of perfluorotributylamine (PFTBA) for EI-GCMS, and leucine enkephalin for ESI-LCMS. The utilized software calibrates the instruments and reports measurements by use of neutral atomic masses. The mass of the electron is not included. IR spectra were recorded on a Perkin Elmer Spectrum Two FT-IR using either neat oil or solid products. Melting points $\left({ }^{\circ} \mathrm{C}\right)$ are uncorrected. The enantiomeric excess was determined using a Supercritical Fluid 
Chromatography (SFC) System. The enantiomeric excess of $\mathbf{4 a}$ was determined using CHIRALPAK OJ3 column.

\subsection{Chemicals}

All reagents were purchased and used as received from suppliers unless otherwise noted. Tetrahydrofuran (THF), $\mathrm{CH}_{2} \mathrm{Cl}_{2}$, and toluene (tol) were dried by passing through alumina cartridges in a solvent purification system. Other dried solvents were purchased from commercial sources and used as received. Deuterated NMR solvents were purchased and stored over $4 \AA$ molecular sieves (MS). Nickel pre-catalysts were synthesized from $\mathrm{NiBr}_{2} \cdot 3 \mathrm{H}_{2} \mathrm{O}$ and bipyridine ligands in $\mathrm{EtOH}$ in analogy to a published

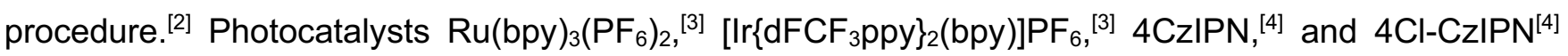
were prepared in-house by the procedure outlined in previous publications; others were purchased from commercial sources. Aryl- and heteroaryl halides were purchased from commercial sources. The preparation of 1-methyl-4-((trimethylsilyl)methyl)piperazine followed a literature procedure. ${ }^{[5]}$ All other preparations of $\alpha$-silylamines are described herein. Bases $\left(\mathrm{Na}_{2} \mathrm{CO}_{3}, \mathrm{~K}_{2} \mathrm{CO}_{3}, \mathrm{Cs}_{2} \mathrm{CO}_{3}, \mathrm{~K}_{3} \mathrm{PO}_{4}, \mathrm{Na}_{2} \mathrm{HPO}_{4}\right.$, $\mathrm{NaOH}, \mathrm{KOH}$ and $t$-BuOK) were purchased and used after grinding with a pestle and mortar and drying under reduced pressure at $60^{\circ} \mathrm{C}$ overnight. 


\section{Synthesis of $\alpha$-Silylamines}

\subsection{General Procedure A}

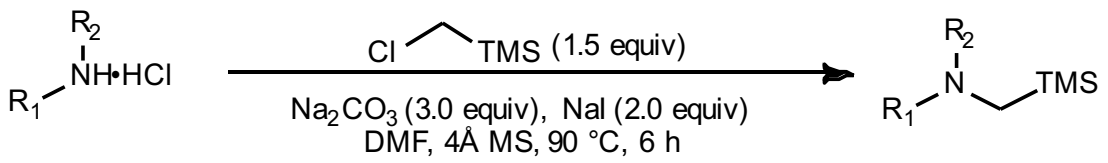

$$
\begin{aligned}
& \mathrm{R}_{1}=\mathrm{H} \text {, alkyl, aryl } \\
& \mathrm{R}_{2}=\mathrm{H} \text {, alkyl }
\end{aligned}
$$

To an appropriately sized round bottom flask equipped with a stir bar was added the amine (1.0 equiv), $\mathrm{Na}_{2} \mathrm{CO}_{3}$ (3.0 equiv), Nal (2.0 equiv), and $4 \AA$ molecular sieves ( $5 \mathrm{wt} \%$ ). The flask was charged with DMF $(0.5 \mathrm{M})$, and the flask was sparged with Ar via a glass adapter and then cooled to $0{ }^{\circ} \mathrm{C}$ via an ice-water bath. After $10 \mathrm{~min}$, chloromethyltrimethylsilane (1.5 equiv) was added dropwise. After completion, the flask was removed from the ice-water bath and heated to $90{ }^{\circ} \mathrm{C}$ using an oil bath. After the reaction achieved full conversion as monitored by LC/MS and TLC, the reaction mixture was cooled to rt and was then filtered through a pad of Celite ${ }^{\circledR}$. The filtrate was transferred to an appropriately sized separatory funnel and diluted with deionized $\mathrm{H}_{2} \mathrm{O}(100 \mathrm{~mL})$ then washed with $\mathrm{Et}_{2} \mathrm{O}(3 \times 30 \mathrm{~mL})$. The combined organic layers were washed with brine $(30 \mathrm{~mL})$. The organic layer was dried $\left(\mathrm{Na}_{2} \mathrm{SO}_{4}\right)$, filtered, and concentrated under reduced pressure. The crude material was purified either through $\mathrm{SiO}_{2}$ chromatography or vacuum distillation. $\mathrm{SiO}_{2}$ chromatography was performed on an automated flash chromatography system, monitoring with a UV detector at $254 \mathrm{~nm}, 280 \mathrm{~nm}$, and 200-300 nm (Threshold: $0.20 \mathrm{AU}$, signal gain $1 \mathrm{x}$ ), and an ELSD (Threshold: $0.05 \mathrm{~V}$, Spray Temperature: $30^{\circ} \mathrm{C}$, Drift Temperature: $60{ }^{\circ} \mathrm{C}$, signal gain $1 \mathrm{x}$ ). The column used was a 40 -gram RediSep ${ }^{\circledR} \mathrm{Rf}$ silica gel disposable flash column

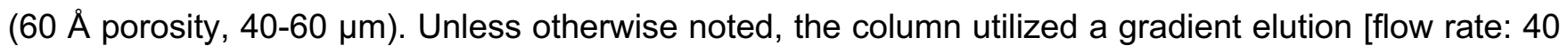
$\mathrm{mL} / \mathrm{min}$, hexanes (Solvent A), EtOAc (Solvent B)]: $5 \min (100 \% \mathrm{~A}), 20 \mathrm{~min}(100 \% \mathrm{~A}$ to $50 \% \mathrm{~A}: 50 \% \mathrm{~B})$, $5 \min ($ Hold $50 \%$ A: $50 \%$ B). 


\subsection{General Procedure B}

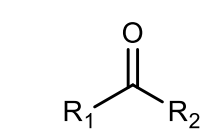

$\mathrm{R}_{1}=\mathrm{H}$, alkyl, aryl
1) $\mathrm{H}_{2} \mathrm{~N} \overbrace{\mathrm{TMS}}(1.0$ equiv)

$\mathrm{Et}_{2} \mathrm{O}, 4 \AA \mathrm{MS}$, rt, overnight

$\mathrm{NaBH}_{4}(2.0$ equiv)

$\mathrm{MeOH}, \mathrm{O}^{\circ} \mathrm{C}$ to $\mathrm{rt}$, overnight

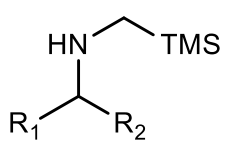

Step 1. To a $100 \mathrm{~mL}$ round bottom flask equipped with a stir bar was added aminomethyltrimethylsilane ( $0.85 \mathrm{~g}, 1.1 \mathrm{~mL}, 10 \mathrm{mmol}, 1.0$ equiv), the corresponding aldehyde or ketone (10 mmol, 1.0 equiv), and $4 \AA$ molecular sieves $(4.0 \mathrm{~g})$ in $\mathrm{Et}_{2} \mathrm{O}(30 \mathrm{~mL}, 0.33 \mathrm{M})$. The reaction mixture was stirred at rt overnight. Upon completion, the soln was filtered, and the solvent was removed under reduced pressure, affording the corresponding imine, which was used without further purification.

Step 2. To a $100 \mathrm{~mL}$ round bottom flask equipped with a stir bar was added the corresponding imine in $\mathrm{MeOH}(50 \mathrm{~mL}, 0.2 \mathrm{M})$. The solution was then cooled to $0 \mathrm{C}^{\circ}$ and stirred for $10 \mathrm{~min}$ at this temperature. After this time, $\mathrm{NaBH}_{4}(0.76 \mathrm{~g}, 20 \mathrm{mmol}, 2$ equiv) was added to the flask portion-wise over $5 \mathrm{~min}$. The reaction mixture was stirred at $0{ }^{\circ} \mathrm{C}$ for an additional $10 \mathrm{~min}$, then warmed to rt overnight. The reaction mixture was then quenched with $\mathrm{H}_{2} \mathrm{O}(30 \mathrm{~mL})$ and $\mathrm{Na}_{2} \mathrm{CO}_{3}(0.756 \mathrm{~g})$ and transferred to a separatory funnel. $\mathrm{CH}_{2} \mathrm{Cl}_{2}(100 \mathrm{~mL})$ was added, and the layers were separated. The aqueous layer was extracted with $\mathrm{CH}_{2} \mathrm{Cl}_{2}(2 \times 50 \mathrm{~mL})$. The combined organic layers were dried $\left(\mathrm{MgSO}_{4}\right)$, filtered, and taken to dryness. Flash chromatography was performed on an automated flash chromatography system, monitoring with a UV detector at $254 \mathrm{~nm}, 280 \mathrm{~nm}$, and 200-300 nm (Threshold: $0.20 \mathrm{AU}$, signal gain 1x), and an ELSD (Threshold: $0.05 \mathrm{~V}$, Spray Temperature: $30^{\circ} \mathrm{C}$, Drift Temperature: $60^{\circ} \mathrm{C}$, signal gain $1 \mathrm{x}$ ). The column used was a 40-gram RediSep ${ }^{\circledR}$ Rf silica gel disposable flash column (60 A porosity, 40-60 $\mu \mathrm{m}$ ). Unless otherwise noted, the column utilized a gradient elution [flow rate: $40 \mathrm{~mL} / \mathrm{min}$, hexanes (Solvent A), EtOAc (Solvent B)]: $5 \min (100 \%$ A), $20 \min (100 \%$ A to $50 \%$ A: $50 \%$ B), 5 min (Hold $50 \%$ A: $50 \%$ B). 


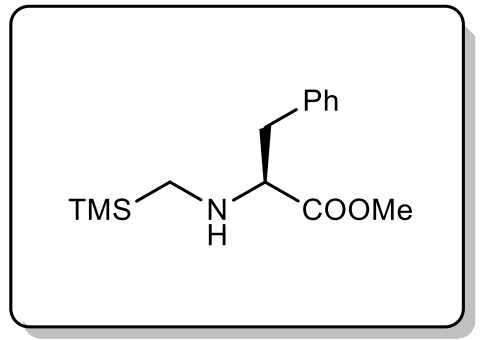

Methyl ((Trimethylsilyl)methyl)-L-phenylalaninate (1a) $(46 \mathrm{mmol}$ scale, $12.3 \mathrm{~g}, 83 \%$ ) was prepared following General Procedure A and purified by vacuum distillation ( $\mathrm{bp}=128-130{ }^{\circ} \mathrm{C} @ 500$ mTorr). The product was obtained as a colorless oil. ${ }^{1} \mathrm{H}$ NMR $\left(400 \mathrm{MHz}, \mathrm{CDCl}_{3}\right) \delta 7.33$ - $7.25(\mathrm{~m}, 2 \mathrm{H}), 7.24-7.20(\mathrm{~m}, 1 \mathrm{H}), 7.17(\mathrm{dd}, J=8.2,1.5 \mathrm{~Hz}, 2 \mathrm{H}), 3.63(\mathrm{~s}, 3 \mathrm{H}), 3.43(\mathrm{t}, J=7.0 \mathrm{~Hz}, 1 \mathrm{H})$, $3.00-2.86(\mathrm{~m}, 2 \mathrm{H}), 2.04(\mathrm{~d}, J=13.2 \mathrm{~Hz}, 1 \mathrm{H}), 1.91(\mathrm{~d}, J=13.2 \mathrm{~Hz}, 1 \mathrm{H}), 1.30(\mathrm{br}, 1 \mathrm{H}),-0.00(\mathrm{~s}, 9 \mathrm{H}) .{ }^{13} \mathrm{C}$ NMR $\left(101 \mathrm{MHz}, \mathrm{CDCl}_{3}\right) \delta 175.3,137.8,129.3,128.4,126.7,67.0,51.6,39.4,38.3,-2.6$. FT-IR $\left(\mathrm{cm}^{-1}\right.$, neat, ATR) 2952, 1496, 1455, 1434, 1349, 1248, 1216, 1193, 1167, 1126, 1080, 984, 748. HRMS (ESITOF) calcd for $\left(\mathrm{C}_{14} \mathrm{H}_{24} \mathrm{NO}_{2} \mathrm{Si}\right)[\mathrm{M}+\mathrm{H}]^{+} 266.1576$, found 266.1565 .

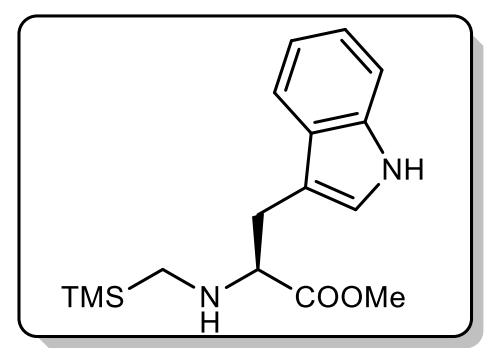

Methyl ((Trimethylsilyl)methyl)-L-tryptophanate (1b) (4 mmol scale, $1.2 \mathrm{~g}, 66 \%$ ) was prepared following General Procedure A and purified by column chromatography. The product was obtained as a pale-yellow solid $\left(\mathrm{mp}=59-60{ }^{\circ} \mathrm{C}\right) .{ }^{1} \mathrm{H}$ NMR $\left(500 \mathrm{MHz}, \mathrm{CDCl}_{3}\right) \delta 8.25(\mathrm{~s}, 1 \mathrm{H}), 7.64$ $(\mathrm{d}, J=7.9 \mathrm{~Hz}, 1 \mathrm{H}), 7.33(\mathrm{~d}, J=8.3 \mathrm{~Hz}, 1 \mathrm{H}), 7.19(\mathrm{t}, J=7.6 \mathrm{~Hz}, 1 \mathrm{H}), 7.12$ (t, $J=7.5 \mathrm{~Hz}, 1 \mathrm{H}), 7.03(\mathrm{~s}, 1 \mathrm{H}), 3.64(\mathrm{~s}, 3 \mathrm{H}), 3.57(\mathrm{t}, J=6.7 \mathrm{~Hz}, 1 \mathrm{H}), 3.22-3.09(\mathrm{~m}, 2 \mathrm{H}), 2.10(\mathrm{~d}, J=$ 13.2 Hz, 1H), 1.97 (d, $J=13.3 \mathrm{~Hz}, 1 \mathrm{H}), 1.50(\mathrm{~s}, 1 \mathrm{H}), 0.01(\mathrm{~s}, 9 \mathrm{H}) .{ }^{13} \mathrm{C}$ NMR (126 MHz, CDCl $) \delta 175.7$, 136.3, 127.7, 122.9, 122.1, 119.5, 119.0, 111.7, 111.3, 66.3, 51.7, 38.5, 29.0, -2.5. FT-IR (cm ${ }^{-1}$, neat, ATR) $3100,1744,1457,1434,1351,1281,1255,1232$, 1210, 1198, 1172, 1113, 1103, 789, 759. HRMS (ESI-TOF) calcd for $\left(\mathrm{C}_{16} \mathrm{H}_{25} \mathrm{~N}_{2} \mathrm{O}_{2} \mathrm{Si}\right)[\mathrm{M}+\mathrm{H}]^{+} 305.1685$, found 305.1685. 


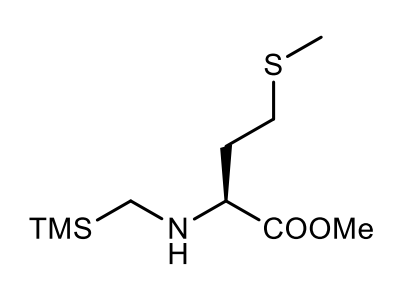

Methyl ((Trimethylsilyl)methyl)-L-methioninate (1c) (8 mmol scale, 1.0 g, 54\%) was prepared following General Procedure A and purified by column chromatography. The product was obtained as a pale-yellow oil. ${ }^{1} \mathrm{H}$ NMR $\left(500 \mathrm{MHz}, \mathrm{CDCl}_{3}\right) \delta 3.68(\mathrm{~s}, 3 \mathrm{H}), 3.26(\mathrm{t}, J=6.9 \mathrm{~Hz}, 1 \mathrm{H}), 2.61-$ $2.48(\mathrm{~m}, 2 \mathrm{H}), 2.05(\mathrm{~s}, 3 \mathrm{H}), 1.98(\mathrm{~d}, J=13.1 \mathrm{~Hz}, 1 \mathrm{H}), 1.93-1.85(\mathrm{~m}, 1 \mathrm{H}), 1.85(\mathrm{~d}, J=12.0 \mathrm{~Hz}, 1 \mathrm{H}), 1.82$ $-1.71(\mathrm{~m}, 1 \mathrm{H}), 1.21(\mathrm{br}, 1 \mathrm{H}),-0.00(\mathrm{~s}, 9 \mathrm{H}) .{ }^{13} \mathrm{C}$ NMR $\left(126 \mathrm{MHz}, \mathrm{CDCl}_{3}\right) \delta$ 175.8, 64.0, 51.7, 38.2, 32.5, 30.8, 15.6, -2.6. FT-IR ( $\mathrm{cm}^{-1}$, neat, ATR) 2952, 2917, 2852, 2789, 1434, 1345, 1298, 1277, 1247, 1192 , 1168, 1125, 987, 761. HRMS (ESI-TOF) calcd for $\left(\mathrm{C}_{10} \mathrm{H}_{24} \mathrm{NO}_{2} \mathrm{SiS}\right)[\mathrm{M}+\mathrm{H}]^{+} 250.1297$, found 250.1291.

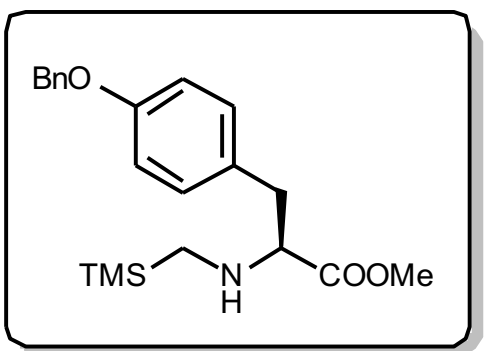

Methyl (S)-3-(4-(Benzyloxy)phenyl)-2-(((trimethylsilyl)methyl)amino) Propanoate (1d) (3 mmol scale, $0.68 \mathrm{~g}, 59 \%$ ) was prepared following General Procedure A and purified by column chromatography. The product was obtained as a white solid $\left(\mathrm{mp}=46-48^{\circ} \mathrm{C}\right) .{ }^{1} \mathrm{H}$ NMR $(500$ $\left.\mathrm{MHz}, \mathrm{CDCl}_{3}\right) \delta 7.42(\mathrm{~d}, J=7.4 \mathrm{~Hz}, 2 \mathrm{H}), 7.38(\mathrm{t}, J=7.5 \mathrm{~Hz}, 2 \mathrm{H}), 7.32(\mathrm{t}, J$ $=7.3 \mathrm{~Hz}, 1 \mathrm{H}), 7.10(\mathrm{~d}, J=8.3 \mathrm{~Hz}, 2 \mathrm{H}), 6.89(\mathrm{~d}, J=8.5 \mathrm{~Hz}, 2 \mathrm{H}), 5.04(\mathrm{~s}, 2 \mathrm{H}), 3.64(\mathrm{~s}, 3 \mathrm{H}), 3.44(\mathrm{~s}, 1 \mathrm{H})$, $2.93(\mathrm{~s}, 2 \mathrm{H}), 2.05$ (d, J = $13.2 \mathrm{~Hz}, 1 \mathrm{H}), 1.97(\mathrm{~d}, J=13.4 \mathrm{~Hz}, 1 \mathrm{H}), 1.59$ (br, 1H), $0.03(\mathrm{~s}, 9 \mathrm{H}) .{ }^{13} \mathrm{C}$ NMR $\left(126 \mathrm{MHz}, \mathrm{CDCl}_{3}\right) \delta 175.3,157.7,137.3,130.3,130.1,128.7,128.0,127.6,114.9,70.2,67.1,51.6,38.5$, 38.3, -2.5. FT-IR (cm ${ }^{-1}$, neat, ATR) 2950, 1733, 1511, 1433, 1386, 1297, 1204, 1190, 1177, 1159, 1126, 1016, 812, 751, 741. HRMS (ESI-TOF) calcd for $\left(\mathrm{C}_{21} \mathrm{H}_{30} \mathrm{NO}_{3} \mathrm{Si}\right)[\mathrm{M}+\mathrm{H}]^{+} 372.1995$, found 372.2009.

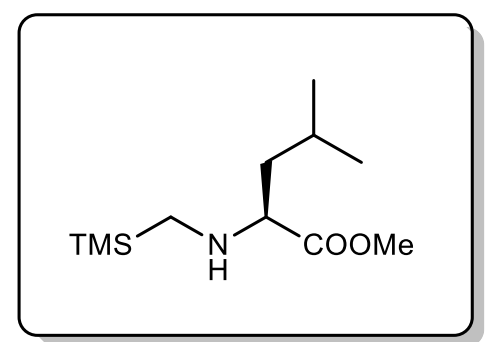

Methyl ((Trimethylsilyl)methyl)-L-leucinate (1e) (5.5 mmol scale, 0.43 g, 34\%) was prepared following General Procedure A and purified by column chromatography. The product was obtained as a pale-yellow oil. ${ }^{1} \mathrm{H}$ NMR $\left(500 \mathrm{MHz}, \mathrm{CDCl}_{3}\right) \delta 3.69(\mathrm{~s}, 3 \mathrm{H}), 3.19(\mathrm{t}, J=7.3 \mathrm{~Hz}, 1 \mathrm{H}), 1.98$ (d, $J=13.2 \mathrm{~Hz}, 1 \mathrm{H}), 1.87(\mathrm{~d}, J=13.3 \mathrm{~Hz}, 1 \mathrm{H}), 1.74-1.64(\mathrm{~m}, 1 \mathrm{H}), 1.42$ 
$(\mathrm{t}, J=7.4 \mathrm{~Hz}, 2 \mathrm{H}), 1.14(\mathrm{br}, 1 \mathrm{H}), 0.90(\mathrm{~d}, J=6.7 \mathrm{~Hz}, 3 \mathrm{H}), 0.87(\mathrm{~d}, J=6.6 \mathrm{~Hz}, 3 \mathrm{H}), 0.01(\mathrm{~s}, 9 \mathrm{H}) .{ }^{13} \mathrm{C}$ NMR $\left(126 \mathrm{MHz}, \mathrm{CDCl}_{3}\right) \delta 176.8,64.0,51.5,42.6,38.3,25.2,22.8,22.6,-2.6$. FT-IR $\left(\mathrm{cm}^{-1}\right.$, neat, ATR) 2954, 2871, 1468, 1434, 1368, 1336, 1309, 1248, 1192, 1166, 1146, 1015, 980, 761. HRMS (ESI-TOF) calcd for $\left(\mathrm{C}_{11} \mathrm{H}_{26} \mathrm{NO}_{2} \mathrm{Si}\right)[\mathrm{M}+\mathrm{H}]^{+} 232.1733$, found 232.1694.

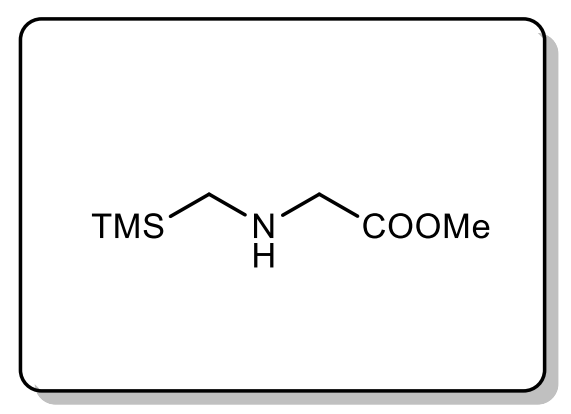

Methyl ((Trimethylsilyl)methyl)glycinate (1f) (8 mmol scale, $1.2 \mathrm{~g}$, $86 \%$ ) was prepared following General Procedure A and purified by column chromatography. The product was obtained as a pale-yellow oil. ${ }^{1} \mathrm{H}$ NMR $\left(500 \mathrm{MHz}, \mathrm{CDCl}_{3}\right) \delta 3.67$ (s, 3H), 3.35 (s, 2H), 2.01 (s, 2H), $1.40(\mathrm{br}, 1 \mathrm{H}), 0.01(\mathrm{~s}, 9 \mathrm{H}) .{ }^{13} \mathrm{C}$ NMR $\left(126 \mathrm{MHz}, \mathrm{CDCl}_{3}\right) \delta 173.1$, 54.8, 51.6, 40.3, -2.6. FT-IR (cm-1, neat, ATR) 3346, 2954, 2900, 2790, 1436, 1347, 1170, 1138, 1052, 986, 941, 767. HRMS (ESI-TOF) calcd for $\left(\mathrm{C}_{7} \mathrm{H}_{18} \mathrm{NO}_{2} \mathrm{Si}\right)[\mathrm{M}+\mathrm{H}]^{+} 176.1107$, found 176.1102 .

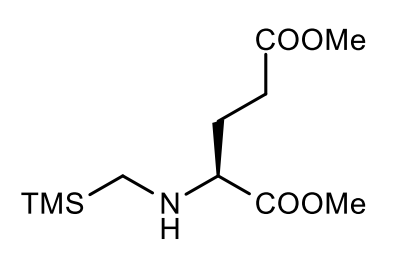

Dimethyl ((Trimethylsilyl)methyl)-L-glutamate $(1 \mathrm{~g})(4.7 \mathrm{mmol}$ scale, $0.30 \mathrm{~g}, 24 \%$ ) was prepared following General Procedure A and purified by column chromatography. The product was obtained as a pale-yellow oil. ${ }^{1} \mathrm{H}$ NMR $\left(500 \mathrm{MHz}, \mathrm{CDCl}_{3}\right) \delta 3.72(\mathrm{~s}, 3 \mathrm{H}), 3.66(\mathrm{~s}, 3 \mathrm{H}), 3.16(\mathrm{dd}, \mathrm{J}=$ 8.4, $5.5 \mathrm{~Hz}, 1 \mathrm{H}), 2.50-2.36(\mathrm{~m}, 2 \mathrm{H}), 2.00(\mathrm{~d}, J=13.2 \mathrm{~Hz}, 1 \mathrm{H}), 2.03-1.91(\mathrm{~m}, 1 \mathrm{H}), 1.91-1.79(\mathrm{~m}, 1 \mathrm{H})$, $1.85(\mathrm{~d}, J=13.0 \mathrm{~Hz}, 1 \mathrm{H}), 1.21(\mathrm{br}, 1 \mathrm{H}), 0.02(\mathrm{~s}, 9 \mathrm{H}) .{ }^{13} \mathrm{C}$ NMR $\left(126 \mathrm{MHz}, \mathrm{CDCl}_{3}\right) \delta 175.7,173.9,64.5$, 51.8, 51.7, 38.2, 30.7, 27.9, -2.6. FT-IR (cm ${ }^{-1}$, neat, ATR) 3355, 2955, 1721, 1521, 1437, 1360, 1295, $1248,1204,1172,1055,987,843,777,733,700,600$. HRMS (ESI-TOF) calcd for $\left(\mathrm{C}_{11} \mathrm{H}_{24} \mathrm{NO}_{4} \mathrm{Si}\right)[\mathrm{M}+\mathrm{H}]^{+}$ 262.1475, found 262.1480 . 


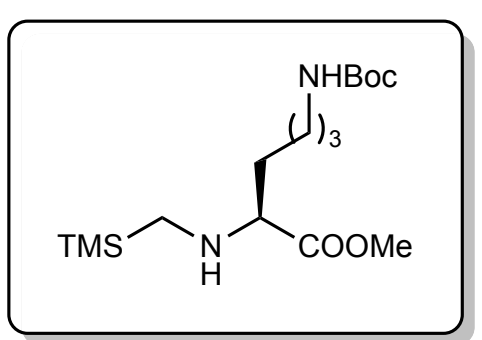

Methyl $\quad N^{6}-\left(\right.$ tert-Butoxycarbonyl)- $N^{2}-(($ trimethylsilyl)methyl)-L-lysinate (1h) $(1.7 \mathrm{mmol}$ scale, $0.48 \mathrm{~g}, 82 \%)$ was prepared following General Procedure A and purified by column chromatography. The product was obtained as a colorless oil. ${ }^{1} \mathrm{H}$ NMR $\left.\left(600 \mathrm{MHz}^{\mathrm{CDCl}}\right)_{3}\right) \delta 4.54(\mathrm{br}, 1 \mathrm{H})$,

$3.71(\mathrm{~s}, 3 \mathrm{H}), 3.13(\mathrm{t}, J=6.8 \mathrm{~Hz}, 1 \mathrm{H}), 3.09(\mathrm{t}, J=6.8 \mathrm{~Hz}, 2 \mathrm{H}), 1.97(\mathrm{~d}, J=13.2 \mathrm{~Hz}, 1 \mathrm{H}), 1.89(\mathrm{~d}, J=13.2$ $\mathrm{Hz}, 1 \mathrm{H}), 1.66-1.53(\mathrm{~m}, 2 \mathrm{H}), 1.49-1.43(\mathrm{~m}, 2 \mathrm{H}), 1.43(\mathrm{~s}, 9 \mathrm{H}), 1.40-1.27(\mathrm{~m}, 2 \mathrm{H}), 1.25(\mathrm{br}, 1 \mathrm{H}), 0.02$ (s, 9H). ${ }^{13} \mathrm{C}$ NMR $\left(151 \mathrm{MHz}, \mathrm{CDCl}_{3}\right) \delta 176.3,156.1,79.2,65.3,51.6,40.5,38.3,32.8,30.0,28.6,23.3$, -2.6. FT-IR ( $\mathrm{cm}^{-1}$, neat, ATR) 3363, 2952, 2787, 1737, 1714, 1520, 1455, 1392, 1366, 1248, 1169, 1041 , 991, 860, 841, 700, 607. HRMS (ESI-TOF) calcd for $\left(\mathrm{C}_{16} \mathrm{H}_{35} \mathrm{~N}_{2} \mathrm{O}_{4} \mathrm{Si}\right)[\mathrm{M}+\mathrm{H}]^{+} 347.2366$, found 347.2363.

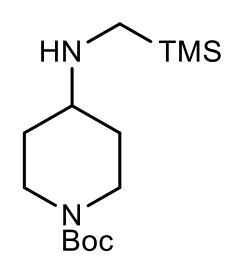

tert-Butyl 4-(((Trimethylsilyl)methyl)amino)piperidine-1-carboxylate (1i) $(25.0 \mathrm{mmol}$ scale, $3.5 \mathrm{~g}, 46 \%)$ was prepared following General Procedure A and purified by vacuum distillation (bp 111-115 ${ }^{\circ} \mathrm{C} @ 500$ mTorr). The product was obtained as a colorless oil. ${ }^{1} \mathrm{H}$ NMR $(500 \mathrm{MHz}$, $\left.\mathrm{CDCl}_{3}\right) \delta 3.93-3.89(\mathrm{~m}, 2 \mathrm{H}), 2.75-2.68(\mathrm{~m}, 2 \mathrm{H}), 2.43-2.32(\mathrm{~m}, 1 \mathrm{H}), 1.96(\mathrm{~s}, 2 \mathrm{H}), 1.77-1.71(\mathrm{~m}, 2 \mathrm{H})$, $1.35(\mathrm{~s}, 9 \mathrm{H}), 1.18-1.07(\mathrm{~m}, 2 \mathrm{H}), 0.48(\mathrm{br}, 1 \mathrm{H}),-0.06(\mathrm{~s}, 9 \mathrm{H}) .{ }^{13} \mathrm{C}$ NMR $\left(126 \mathrm{MHz}, \mathrm{CDCl}_{3}\right) \delta 154.8,79.2$, 58.6, 42.9, 36.8, 28.5, -2.5. HRMS (ESI-TOF) calcd for $\left(\mathrm{C}_{14} \mathrm{H}_{31} \mathrm{~N}_{2} \mathrm{O}_{2} \mathrm{Si}\right)[\mathrm{M}+\mathrm{H}]^{+}$287.2155, found 287.2153.

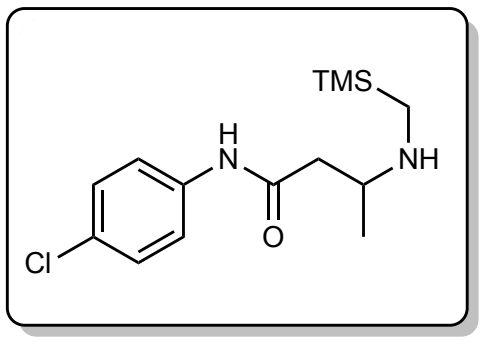

N-(4-Chlorophenyl)-3-(((trimethylsilyl)methyl)amino)butanamide (1j) (10 mmol scale, $1.4 \mathrm{~g}, 48 \%$ ) was prepared following General Procedure B. The product was obtained as a pale-yellow solid $\left(\mathrm{mp}=52-53^{\circ} \mathrm{C}\right) .{ }^{1} \mathbf{H}$ NMR $\left(500 \mathrm{MHz}, \mathrm{CDCl}_{3}\right) \delta 11.02(\mathrm{~s}, 1 \mathrm{H}), 7.47(\mathrm{~d}, J=8.8 \mathrm{~Hz}, 2 \mathrm{H}), 7.25$ (d, $J=9.2 \mathrm{~Hz}, 2 \mathrm{H}), 3.00(\mathrm{td}, J=6.7,3.2 \mathrm{~Hz}, 1 \mathrm{H}), 2.64(\mathrm{dd}, J=16.5,3.2 \mathrm{~Hz}$, 1H), $2.27(\mathrm{dd}, J=16.5,6.9 \mathrm{~Hz}, 1 \mathrm{H}), 2.16(\mathrm{~d}, J=13.5 \mathrm{~Hz}, 1 \mathrm{H}), 2.10(\mathrm{~d}, J=13.3 \mathrm{~Hz}, 1 \mathrm{H}), 1.21(\mathrm{~d}, J=6.5$ 
$\mathrm{Hz}, 3 \mathrm{H}), 0.87$ (br, 1H), 0.11 (s, 9H). ${ }^{13} \mathrm{C}$ NMR (126 MHz, $\left.\mathrm{CDCl}_{3}\right) \delta 170.5,137.4,129.0,128.5,121.2$, 54.1, 40.9, 36.4, 20.0, -2.4. FT-IR (cm ${ }^{-1}$, neat, ATR) 2956, 1665, 1601, 157, 1491, 1468, 1400, 1369 , 1338, 1307, 1249, 1093, 835, 811, 772, 757, 743. HRMS (ESI-TOF) calcd for $\left(\mathrm{C}_{14} \mathrm{H}_{24} \mathrm{~N}_{2} \mathrm{OSiCl}\right)[\mathrm{M}+\mathrm{H}]^{+}$ 299.1346, found 299.1337.

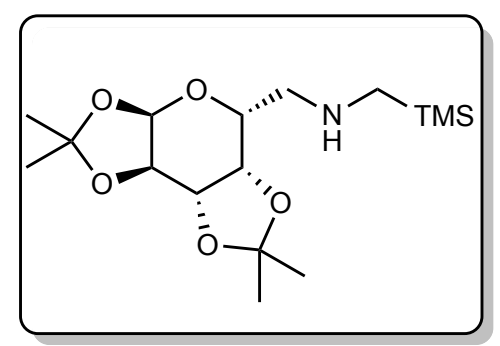

1-((3aR,5R,5aS,8aS,8bR)-2,2,7,7-Tetramethyltetrahydro-5H-bis([1,3] dioxolo)[4,5-b:4',5'-d]pyran-5-yl)-N-((trimethylsilyl)methyl)methanamine (1k) (10 mmol scale, $2.1 \mathrm{~g}, 60 \%)$ was prepared following General Procedure B. The product was obtained as a solid $\left(\mathrm{mp}=45-47^{\circ} \mathrm{C}\right)$.

${ }^{1} \mathrm{H}$ NMR $\left(500 \mathrm{MHz}, \mathrm{CDCl}_{3}\right) \delta 5.51(\mathrm{~d}, J=5.1 \mathrm{~Hz}, 1 \mathrm{H}), 4.57(\mathrm{dd}, J=7.9,2.5 \mathrm{~Hz}, 1 \mathrm{H}), 4.31-4.26(\mathrm{~m}, 1 \mathrm{H})$, $4.17-4.12(\mathrm{~m}, 1 \mathrm{H}), 3.98-3.93(\mathrm{~m}, 1 \mathrm{H}), 2.91(\mathrm{dd}, J=12.8,8.7 \mathrm{~Hz}, 1 \mathrm{H}), 2.71(\mathrm{dd}, J=13.0,3.9 \mathrm{~Hz}, 1 \mathrm{H})$, $2.17-2.10(\mathrm{~m}, 1 \mathrm{H}), 2.04-1.96(\mathrm{~m}, 1 \mathrm{H}), 1.52(\mathrm{~s}, 3 \mathrm{H}), 1.43(\mathrm{~s}, 3 \mathrm{H}), 1.31(\mathrm{~s}, 6 \mathrm{H}), 1.16(\mathrm{br}, 1 \mathrm{H}), 0.02(\mathrm{~s}$, 9H). ${ }^{13} \mathrm{C}$ NMR $\left(126 \mathrm{MHz}, \mathrm{CDCl}_{3}\right) \delta 109.3,108.5,96.5,72.3,71.0,70.8,66.0,53.7,39.8,26.2,26.1,25.1$, 24.5, -2.4. FT-IR ( $\mathrm{cm}^{-1}$, neat, ATR) 3000, 1383, 1372, 1173, 1143, 959, 919, 900, 806, 771, 731. HRMS (ESI-TOF) calcd for $\left(\mathrm{C}_{16} \mathrm{H}_{32} \mathrm{NO}_{5} \mathrm{Si}\right)[\mathrm{M}+\mathrm{H}]^{+} 346.2050$, found 346.2038.

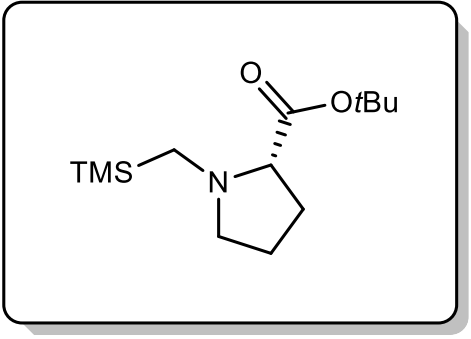

tert-Butyl ((Trimethylsilyl)methyl)-L-prolinate (1I) (5 mmol scale, $1.2 \mathrm{~g}$, 90\%) was prepared following General Procedure A and purified by column chromatography. The product was obtained as a pale-yellow oil. ${ }^{1} \mathrm{H}$ NMR $\left(500 \mathrm{MHz}, \mathrm{CDCl}_{3}\right) \delta 3.07(\mathrm{td}, J=8.2,3.5 \mathrm{~Hz}, 1 \mathrm{H}), 2.93$ (dd, $J=$ 8.7, 5.6 Hz, 1H), $2.34(\mathrm{~d}, J=14.2 \mathrm{~Hz}, 1 \mathrm{H}), 2.34-2.29(\mathrm{~m}, 1 \mathrm{H}), 2.06-1.94(\mathrm{~m}, 1 \mathrm{H}), 1.91-1.79(\mathrm{~m}, 2 \mathrm{H})$, $1.83(\mathrm{~d}, J=14.0 \mathrm{~Hz}, 1 \mathrm{H}), 1.80-1.70(\mathrm{~m}, 1 \mathrm{H}), 1.45(\mathrm{~s}, 9 \mathrm{H}), 0.05(\mathrm{~s}, 9 \mathrm{H}) .{ }^{13} \mathrm{C} \mathrm{NMR}\left(126 \mathrm{MHz}, \mathrm{CDCl}_{3}\right) \delta$ 173.5, 80.3, 70.5, 56.1, 45.7, 29.0, 28.3, 23.5, -1.2. FT-IR ( $\mathrm{cm}^{-1}$, neat, ATR) 2956, 2776, 1724, 1479, 1457, 1418, 1391, 1367, 1295, 1248, 1210, 1119, 1032, 763, 692. HRMS (ESI-TOF) calcd for $\left(\mathrm{C}_{13} \mathrm{H}_{28} \mathrm{NO}_{2} \mathrm{Si}\right)[\mathrm{M}+\mathrm{H}]^{+}$258.1889, found 258.1914. 


\subsection{Synthesis of Silylamine $1 \mathrm{~m}$ from Aspartame (1m)}

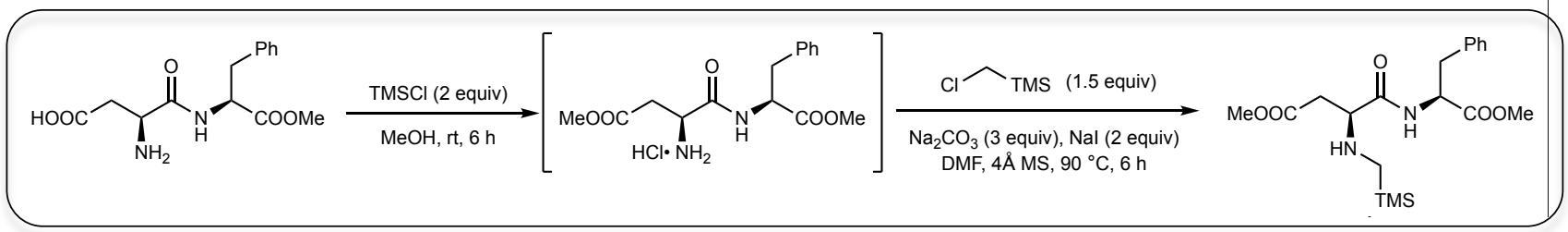

The first step, methyl esterification, used a modified literature procedure ${ }^{[6]}$. To a $25 \mathrm{~mL}$ round bottom flask was added aspartame (200.0 mg, $0.7 \mathrm{mmol})$ and $\mathrm{Me}_{3} \mathrm{SiCl}(148 \mathrm{mg}, 172 \mu \mathrm{L}, 1.4 \mathrm{mmol}$, 2 equiv) was added slowly and stirred with a magnetic stirrer. Then $\mathrm{MeOH}(5 \mathrm{~mL})$ was added slowly over 5 min, and the resulting suspension was stirring at rt for $6 \mathrm{~h}$. After the reaction achieved full conversion as monitored by LC/MS, the reaction mixture was concentrated under reduced pressure and dried under high vacuum overnight. The white flaky crude material was subjected directly to General Procedure A and purified by column chromatography to afford the corresponding silylamine $1 \mathrm{p}(199 \mathrm{mg}, 72 \%)$ as a yellow oil.

${ }^{1} \mathrm{H}$ NMR $\left(400 \mathrm{MHz}, \mathrm{CDCl}_{3}\right) \delta 7.74(\mathrm{~d}, J=8.5 \mathrm{~Hz}, 1 \mathrm{H}), 7.37-7.19(\mathrm{~m}, 3 \mathrm{H}), 7.18-7.07(\mathrm{~m}, 2 \mathrm{H}), 4.88(\mathrm{dt}$, $J=8.5,6.1 \mathrm{~Hz}, 1 \mathrm{H}), 3.73(\mathrm{~s}, 3 \mathrm{H}), 3.69(\mathrm{~s}, 3 \mathrm{H}), 3.36(\mathrm{dd}, J=8.5,3.8 \mathrm{~Hz}, 1 \mathrm{H}), 3.13(\mathrm{qd}, J=13.9,6.1 \mathrm{~Hz}$, 2H), $2.78(\mathrm{dd}, J=16.5,3.8 \mathrm{~Hz}, 1 \mathrm{H}), 2.54(\mathrm{dd}, J=16.5,8.5 \mathrm{~Hz}, 1 \mathrm{H}), 1.96(\mathrm{~d}, J=5.1 \mathrm{~Hz}, 2 \mathrm{H}), 1.63(\mathrm{br}$, 1H), -0.00 (s, 9H). ${ }^{13} \mathrm{C}$ NMR $\left(101 \mathrm{MHz}, \mathrm{CDCl}_{3}\right) \delta 172.5,172.3,171.9,136.1,129.4,128.7,127.2,62.6$, 52.7, 52.3, 51.9, 38.1, 38.0, 35.0, -2.9. FT-IR ( $\mathrm{cm}^{-1}$, neat, ATR) 3346, 2953, 1738, 1674, 1510, 1437, 1361, 1248, 1199, 1174, 1122, 1080, 1020, 995, 858, 841, 744, 700, 609, 493. HRMS (ESI-TOF) calcd for $\left(\mathrm{C}_{19} \mathrm{H}_{30} \mathrm{~N}_{2} \mathrm{O}_{5} \mathrm{NaSi}\right)[\mathrm{M}+\mathrm{Na}]^{+}$417.1822, found 417.1812. 


\subsection{Synthesis of Methyl N-Benzyl-N-((trimethylsilyl)methyl)-L-phenylalaninate (1n)}

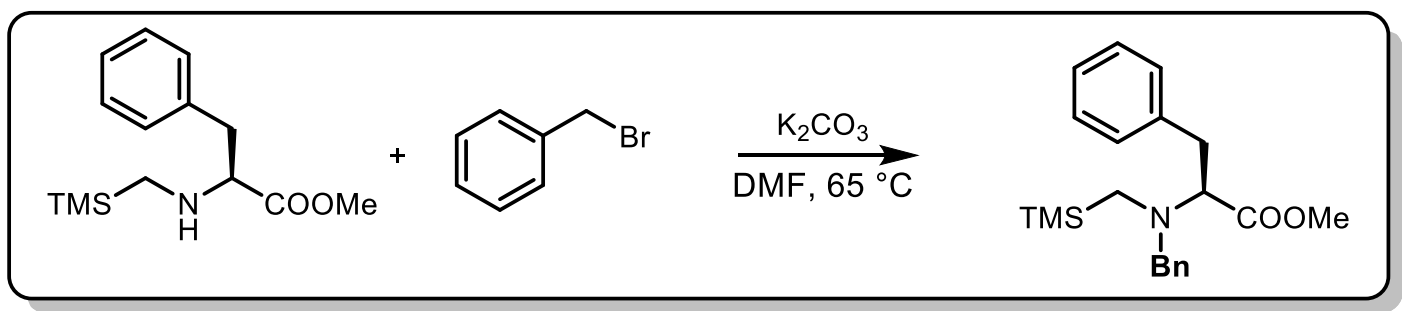

To a $50 \mathrm{~mL}$ round bottom flask was added methyl ((trimethylsilyl)methyl)- L-phenylalaninate (630 mg, 2.4 mmol, 1 equiv) and a magnetic stirrer. The flask was sparged with Ar and later charged with anhyd, degassed DMF $(20 \mathrm{~mL})$. Then the flask was cooled in an ice-water bath for $10 \mathrm{~min}$. After that, $\mathrm{K}_{2} \mathrm{CO}_{3}$ (656 mg, $4.8 \mathrm{mmol}, 2$ equiv) and benzyl bromide (487 mg, $0.34 \mathrm{~mL}, 2.9 \mathrm{mmol}, 1.2$ equiv) were added, and the reaction mixture was kept stirring in an ice-water bath for another $10 \mathrm{~min}$. After this time, the reaction mixture was warmed to $60{ }^{\circ} \mathrm{C}$ using an oil bath. Upon completion, the reaction mixture was cooled to $0{ }^{\circ} \mathrm{C}$ by an ice-water bath and quenched with $\mathrm{H}_{2} \mathrm{O}(5 \mathrm{~mL})$ and the mixture was extracted with $\mathrm{Et}_{2} \mathrm{O}(3 \times 25 \mathrm{~mL})$. The combined organic extracts were washed with brine $(15 \mathrm{~mL})$. The organic layer was dried $\left(\mathrm{Na}_{2} \mathrm{SO}_{4}\right)$, filtered, and concentrated via rotary evaporation. Further purification was accomplished by $\mathrm{SiO}_{2}$ column chromatography: $\mathrm{SiO}_{2}$ chromatography was performed on an automated flash chromatography system, monitoring with a UV detector at $254 \mathrm{~nm}, 280 \mathrm{~nm}$, and 200-300 nm (Threshold: $0.20 \mathrm{AU}$, signal gain 1x), and an ELSD (Threshold: $0.05 \mathrm{~V}$, Spray Temperature: $30^{\circ} \mathrm{C}$, Drift Temperature: $60^{\circ} \mathrm{C}$, signal gain $1 \mathrm{x}$ ). The column used was a 40-gram RediSep Rf Gold ${ }^{\circledR}$ silica gel

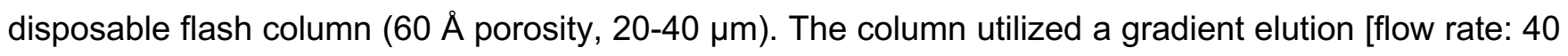
$\mathrm{mL} / \mathrm{min}$, hexanes (Solvent A), EtOAc (Solvent B)]: $3 \min (100 \% \mathrm{~A}), 6 \min (100 \% \mathrm{~A}$ to $80.9 \% \mathrm{~A}: 10.1 \%$ B), $1 \mathrm{~min}$ (Hold $80.9 \%$ A: 10.1\% B). The product 1q was obtained as a colorless oil (841 mg, 99\%)

${ }^{1} \mathrm{H}$ NMR $\left(400 \mathrm{MHz}, \mathrm{CDCl}_{3}\right) \delta 7.28-7.12(\mathrm{~m}, 8 \mathrm{H}), 7.08-7.01(\mathrm{~m}, 2 \mathrm{H}), 3.90(\mathrm{~d}, J=13.9 \mathrm{~Hz}, 1 \mathrm{H}), 3.66$ (s, 3H), $3.52(\mathrm{~d}, J=8.6 \mathrm{~Hz}, 1 \mathrm{H}), 3.50(\mathrm{~d}, J=2.3 \mathrm{~Hz}, 1 \mathrm{H}), 3.03(\mathrm{dd}, J=13.7,8.0 \mathrm{~Hz}, 1 \mathrm{H}), 2.85(\mathrm{dd}, J=$ 13.7, 7.0 Hz, 1H), $2.23(\mathrm{~d}, J=14.8 \mathrm{~Hz}, 1 \mathrm{H}), 1.98(\mathrm{~d}, J=14.7 \mathrm{~Hz}, 1 \mathrm{H}),-0.00(\mathrm{~s}, 9 \mathrm{H}) .{ }^{13} \mathrm{C}$ NMR $(101 \mathrm{MHz}$, $\left.\mathrm{CDCl}_{3}\right) \delta 172.5,139.7,138.7,129.6,128.9,128.3,128.3,127.0,126.3,65.4,58.3,51.0,41.8,35.9,-1.2$. 
SUPPORTING INFORMATION

FT-IR (cm ${ }^{-1}$, neat, ATR) 3029, 2951, 2898, 2839, 1731, 1495, 1454, 1368, 1248, 1208, 1161, 1113, $1075,1029,987,853,838,737,697,539$. HRMS (ESI-TOF) calcd for $\left(\mathrm{C}_{21} \mathrm{H}_{30} \mathrm{NO}_{2} \mathrm{Si}\right)[\mathrm{M}+\mathrm{H}]^{+} 356.2046$, found 356.2034 . 


\subsection{Synthesis of 3-Methyl-1-((trimethylsilyl)methyl)-1H-indole (10)}

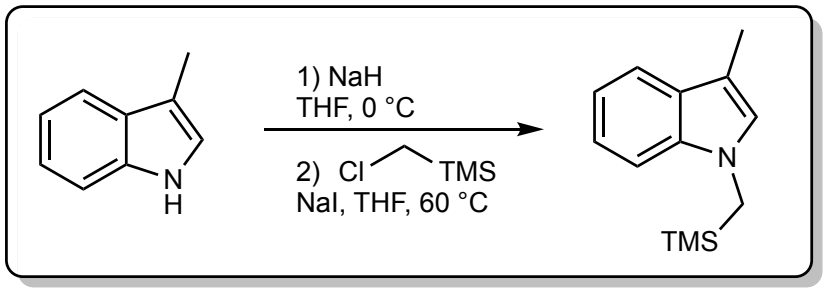

To a $100 \mathrm{~mL}$ round bottom flask was added 3-methyl- $1 H$-indole $(1.000 \mathrm{~g}, 7.6 \mathrm{mmol}, 1$ equiv) and a magnetic stirrer. The flask was sparged with Ar and later charged with anhyd, degassed THF (30 mL). Then the flask was cooled in an ice-water bath for $10 \mathrm{~min}$. After that, $\mathrm{NaH}$ ( $0.366 \mathrm{~g}, 15.3 \mathrm{mmol}, 2$ equiv) was added in two separate batches over $5 \mathrm{~min}$, and the reaction mixture was kept stirring in the icewater bath for another $30 \mathrm{~min}$. After this perios of time, Nal $(1.14 \mathrm{~g}, 7.6 \mathrm{mmol}, 1$ equiv) and chloromethyltrimethylsilane $(0.954 \mathrm{~g}, 7.8 \mathrm{mmol}, 1.02$ equiv) were added, and the reaction mixture was warmed to $60{ }^{\circ} \mathrm{C}$ using an oil bath. Upon completion as monitored by TLC, the reaction mixture was cooled to $0{ }^{\circ} \mathrm{C}$ in an ice-water bath and quenched with satd aq $\mathrm{NH}_{4} \mathrm{Cl}(10 \mathrm{~mL})$, and the mixture was extracted with $\mathrm{Et}_{2} \mathrm{O}(3 \times 25 \mathrm{~mL})$. The combined organic extracts were washed with brine $(25 \mathrm{~mL})$. The organic layer was dried $\left(\mathrm{Na}_{2} \mathrm{SO}_{4}\right)$, filtered, and concentrated via rotary evaporation. Further purification was accomplished by $\mathrm{SiO}_{2}$ column chromatography. $\mathrm{SiO}_{2}$ chromatography was performed on an automated flash chromatography system, monitoring with a UV detector at $254 \mathrm{~nm}, 280 \mathrm{~nm}$, and 200$300 \mathrm{~nm}$ (Threshold: $0.20 \mathrm{AU}$, signal gain 3x), and an ELSD (Threshold: $0.05 \mathrm{~V}$, Spray Temperature: $30{ }^{\circ} \mathrm{C}$, Drift Temperature: $60^{\circ} \mathrm{C}$, signal gain 3x) The column used was a 40-gram RediSep Rf Gold ${ }^{\circledR}$ silica gel disposable flash column (60 Å porosity, 20-40 $\mu \mathrm{m})$. The column utilized a gradient elution [flow rate: $40 \mathrm{~mL} / \mathrm{min}$, hexanes (Solvent A), EtOAc (Solvent B)]: $5 \min (100 \% \mathrm{~A}), 5 \mathrm{~min}(100 \%$ A to 95.2\% A: 4.8\% B), $3 \mathrm{~min}$ (Hold 95.2\% A: 4.8\% B), $2.2 \min (95.2 \%$ A: $4.8 \%$ B to $91.2 \%$ A: $8.8 \%$ B). The desired compound $1 \mathrm{r}$ was obtained as a pale-yellow oil $(1.000 \mathrm{~g}, 60 \%)$

${ }^{1} \mathrm{H}$ NMR $\left(600 \mathrm{MHz}, \mathrm{CDCl}_{3}\right) \delta 7.56(\mathrm{~d}, J=7.9 \mathrm{~Hz}, 1 \mathrm{H}), 7.23(\mathrm{~d}, J=8.3 \mathrm{~Hz}, 1 \mathrm{H}), 7.18$ (ddd, $J=8.2,6.8$, $1.2 \mathrm{~Hz}, 1 \mathrm{H}), 7.07$ (ddd, $J=7.9,6.9,1.0 \mathrm{~Hz}, 1 \mathrm{H}), 6.79(\mathrm{~s}, 1 \mathrm{H}), 3.62(\mathrm{~s}, 2 \mathrm{H}), 2.34(\mathrm{~d}, J=1.0 \mathrm{~Hz}, 3 \mathrm{H}), 0.09$ 
(s, 9H). ${ }^{13} \mathrm{C}$ NMR $\left(151 \mathrm{MHz}, \mathrm{CDCl}_{3}\right) \delta 137.0,128.3,126.2,121.1,118.9,118.1,109.7,109.5,37.2,9.8$, -1.8. FT-IR ( $\mathrm{cm}^{-1}$, neat, ATR) 3356, 2925, 1691, 1611, 1487, 1465, 1356, 1250, 1190, 930, 846, 794, 743, 695. HRMS (EI-TOF) calcd for $\left(\mathrm{C}_{13} \mathrm{H}_{19} \mathrm{NSi}\right)[\mathrm{M}]^{+} 217.1287$, found 217.1280. 


\section{Extended Optimizations and Control Studies}

To a 1-dram reaction vial equipped with a stirrer bar was added electrophile $(0.1 \mathrm{mmol}, 1$ equiv), $\alpha$ silylamine ( $0.15 \mathrm{mmol}, 1.5$ equiv), the appropriate amount of inorganic base, the appropriate amount of photocatalyst, and the appropriate amount of $\mathrm{Ni}(\mathrm{II})$ precomplex. The vial was sealed with a cap containing a TFE-lined silicone septum and was evacuated and purged with argon three times via an inlet needle. The vial was then charged with anhyd degassed solvent $(1 \mathrm{~mL})$. After this, the cap was sealed with Parafilm ${ }^{\circledR}$, and the vial was irradiated with blue LEDs for $20 \mathrm{~h}(470 \mathrm{~nm}, \sim 10 \mathrm{~W}$, at a distance of $\sim 2 \mathrm{~cm}$ ). The temperature of the reaction was maintained at approximately $27^{\circ} \mathrm{C}$ via a fan. After $20 \mathrm{~h}$, 1,3,5-trimethoxybenzene ( $16.8 \mathrm{mg}, 0.1 \mathrm{mmol})$ was added to each vial as an internal standard. The crude mixture was filtered by Celite ${ }^{\circledR} 545$. Then the crude reaction was evaluated by ${ }^{1} \mathrm{H}$ NMR to determine product yield against 1,3,5-trimethoxybenzene.

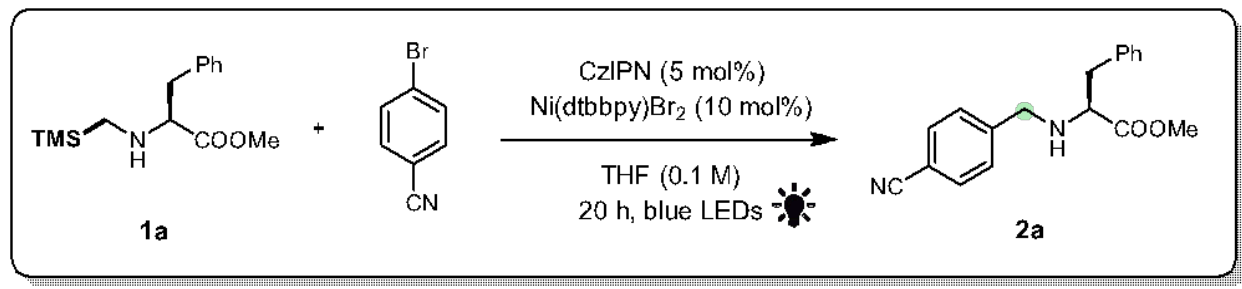

\begin{tabular}{|c|c|c|}
\hline Entry & Variation from condition & NMR yield/\% \\
\hline 1 & none & trace \\
\hline 2 & $\begin{array}{c}\mathrm{Ru}(\mathrm{bpy})_{3}\left(\mathrm{PF}_{6}\right)_{2}(5 \mathrm{~mol} \%) \text { and DMF }(0.1 \mathrm{M}) \text { instead of } \\
\text { 4CzIPN }(5 \mathrm{~mol} \%) \text { and THF }(0.1 \mathrm{M})\end{array}$ & 0 \\
\hline 3 & $\mathrm{Ru}(\mathrm{bpy})_{3}\left(\mathrm{PF}_{6}\right)_{2}(5 \mathrm{~mol} \%)$ instead of $4 \mathrm{CzIPN}$ & 0 \\
\hline 4 & $\operatorname{Ir}\left(\mathrm{dFCF}{ }_{3} \mathrm{ppy}\right)_{2}(\mathrm{dtbbpy}) \mathrm{PF}_{6}(5 \mathrm{~mol} \%)$ instead of $4 \mathrm{CzIPN}$ & trace \\
\hline 5 & $4 \mathrm{Cl}-\mathrm{CzIPN}(5 \mathrm{~mol} \%)$ instead of $4 \mathrm{CzIPN}$ & trace \\
\hline 6 & Adding $\mathrm{K}_{2} \mathrm{CO}_{3}$ (3 equiv) & 15 \\
\hline Entry & Variation from condition 6 & NMR yield/\% \\
\hline 7 & $\mathrm{CsF}$ (3 equiv) instead of $\mathrm{K}_{2} \mathrm{CO}_{3}$ & trace \\
\hline 8 & $\mathrm{~K}_{3} \mathrm{PO}_{4}$ (3 equiv) instead of $\mathrm{K}_{2} \mathrm{CO}_{3}$ & 10 \\
\hline
\end{tabular}


9

10

11

12

13

14

15

16

17

18

19

20

21
$\mathrm{Na}_{2} \mathrm{CO}_{3}$ (3 equiv) instead of $\mathrm{K}_{2} \mathrm{CO}_{3}$

$$
\mathrm{Cs}_{2} \mathrm{CO}_{3} \text { (3 equiv) instead of } \mathrm{K}_{2} \mathrm{CO}_{3}
$$

8

$\mathrm{Na}_{2} \mathrm{HPO}_{4}$ (3 equiv) instead of $\mathrm{K}_{2} \mathrm{CO}_{3}$

12

$\mathrm{NaOH}$ (3 equiv) instead of $\mathrm{K}_{2} \mathrm{CO}_{3}$

0

$\mathrm{KOH}$ (3 equiv) instead of $\mathrm{K}_{2} \mathrm{CO}_{3}$

0

$t$-BuOK ( 3 equiv) instead of $\mathrm{K}_{2} \mathrm{CO}_{3}$

0

$\mathrm{Ni}(\mathrm{dtbbpy}) \mathrm{Br}_{2}(2 \mathrm{~mol} \%)$ instead of $10 \mathrm{~mol} \%$

99

$6 \mathrm{Ni}(\mathrm{bpy}) \mathrm{Br}_{2}(2 \mathrm{~mol} \%)$ instead of $\mathrm{Ni}(\mathrm{dtbbpy}) \mathrm{Br}_{2}(2 \mathrm{~mol} \%)$

94

$\mathrm{Ni}(\mathrm{bdMepy}) \mathrm{Br}_{2}$ (2 mol\%) instead of $\mathrm{Ni}(\mathrm{dtbbpy}) \mathrm{Br}_{2}(2$ $\mathrm{mol} \%)$

91

Blue Kessil LEDs $(2 \times 30 \mathrm{~W})$ instead of Blue LED strip

85

No light

0

No Ni(dtbbpy)Br 2

0

No 4CzIPN

0

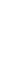
4 


\section{General Procedure for Aminomethylation of Aryl Halides}

General procedure C: To an $8 \mathrm{~mL}$ reaction vial equipped with a stirrer bar was added 4CzIPN (11.8 mg, $5 \mathrm{~mol} \%), \mathrm{Ni}(\mathrm{dtbbpy}) \mathrm{Br}_{2}(2.9 \mathrm{mg}, 2 \mathrm{~mol} \%)$, (hetero)aryl bromide (0.3 mmol, 1.0 equiv), $\alpha$-silylamine (0.45 mmol, 1.5 equiv) and $\mathrm{K}_{2} \mathrm{CO}_{3}(124.4 \mathrm{mg}, 0.9 \mathrm{mmol}$, 3.0 equiv). The reaction vessel was sealed with a cap containing a TFE-lined silicone septum and cooled to $0{ }^{\circ} \mathrm{C}$ in an ice-water bath. Then it was evacuated and backfilled with argon three times. After that, degassed THF $(3 \mathrm{~mL})$ was added, and the vessel was removed from the ice-water bath and further sealed with Parafilm ${ }^{\circledR}$. The vessel was then placed in a sonicator for $3 \mathrm{~min}$ and irradiated with blue LEDs $(470 \mathrm{~nm}, \sim 10 \mathrm{~W}$, at a distance of $\sim 2 \mathrm{~cm})$. When judged complete (typically within $20 \mathrm{~h}$ ), $5 \mathrm{~mL}$ of acetone was added, and the crude material was passed through a pad of Celite ${ }^{\circledR}$ and eluted with another $5 \mathrm{~mL}$ of acetone. The filtrate was concentrated under reduced pressure and purified by $\mathrm{SiO}_{2}$ column chromatography. $\mathrm{SiO}_{2}$ chromatography was performed on an automated flash chromatography system, monitoring with a UV detector at $254 \mathrm{~nm}, 280 \mathrm{~nm}$, and 200$300 \mathrm{~nm}$ (Threshold: $0.20 \mathrm{AU}$, signal gain 3x), and an ELSD (Threshold: $0.05 \mathrm{~V}$, Spray Temperature: $30^{\circ} \mathrm{C}$, Drift Temperature: $60^{\circ} \mathrm{C}$, signal gain 3x). The column used was a 12-gram RediSep ${ }^{\circledR} \mathrm{Rf}$ silica gel disposable flash column (60 $\AA$ porosity, 40-60 $\mu \mathrm{m}$ ). Unless otherwise noted, the column utilized a gradient elution [flow rate: $30 \mathrm{~mL} / \mathrm{min}$, hexanes (Solvent A), EtOAc (Solvent B)]: 2 min (100\% A), 6 min (100\% A to $65 \%$ A: $35 \%$ B), 6 min (Hold 65\% A: 35\% B), 2 min (65\% A: 35\% B to $50 \%$ A: $50 \%$ B), 3 min (Hold $50 \%$ A: $50 \%$ B), 3 min (50\% A: $50 \%$ B to $20 \%$ A: $80 \%$ B), 3 min (Hold $20 \%$ A: $80 \%$ B).

General procedure D: To an $8 \mathrm{~mL}$ reaction vial equipped with a stirrer bar was added 4CzIPN $(7.1 \mathrm{mg}$, $3 \mathrm{~mol} \%), \mathrm{Ni}(\mathrm{dtbbpy}) \mathrm{Br}_{2}(11.7 \mathrm{mg}, 8 \mathrm{~mol} \%)$, (hetero)aryl bromide (0.3 mmol, 1.0 equiv), a-silylamine (0.45 mmol, 1.5 equiv) and $\mathrm{K}_{2} \mathrm{CO}_{3}(124.4 \mathrm{mg}, 0.9 \mathrm{mmol}, 3.0$ equiv). The reaction vessel was sealed with a cap containing a TFE-lined silicone septum and cooled to $0{ }^{\circ} \mathrm{C}$ in an ice-water bath. Then it was evacuated and backfilled with argon three times. After that, degassed THF ( $3 \mathrm{~mL})$ was added, and the vessel was removed from the ice-water bath and further sealed with Parafilm ${ }^{\circledR}$. The vessel was then placed in a sonicator for 3 min and subsequently irradiated with blue LEDs ( $470 \mathrm{~nm}, \sim 10 \mathrm{~W}$, at a distance of $\sim 2 \mathrm{~cm}$ ). When judged complete (typically within $20 \mathrm{~h}$ ), $5 \mathrm{~mL}$ of acetone was added, and the crude 
material was passed through a pad of Celite ${ }^{\circledR}$ and eluted with another $5 \mathrm{~mL}$ of acetone. The filtrate was concentrated under reduced pressure and purified by $\mathrm{SiO}_{2}$ column chromatography. $\mathrm{SiO}_{2}$ chromatography was performed on an automated flash chromatography system, monitoring with a UV detector at $254 \mathrm{~nm}, 280 \mathrm{~nm}$, and 200-300 nm (Threshold: $0.20 \mathrm{AU}$, signal gain 3x), and an ELSD (Threshold: $0.05 \mathrm{~V}$, Spray Temperature: $30{ }^{\circ} \mathrm{C}$, Drift Temperature: $60^{\circ} \mathrm{C}$, signal gain $3 \mathrm{x}$ ). The column used was a 12-gram RediSep ${ }^{\circledR}$ Rf silica gel disposable flash column (60 A porosity, 40-60 $\mu \mathrm{m}$ ). Unless otherwise noted, the column utilized a gradient elution [flow rate: $30 \mathrm{~mL} / \mathrm{min}$, hexanes (Solvent A), EtOAc (Solvent B)]: $1 \min (100 \%$ A), $2 \min (100 \%$ A to $90 \%$ A: 10\% B), $3 \min ($ Hold $90 \%$ A: 10\% B), 6 min $(90 \%$ A: $10 \%$ B to $70 \%$ A: $30 \%$ B), 2 min (Hold $70 \%$ A: $30 \%$ B), 1 min (70\% A: $30 \%$ B to $50 \%$ A: $50 \%$ B), 4 $\min ($ Hold $50 \%$ A: 50\% B), 2 min (50\% A: 50\% B to 20\% A: 80\% B), 4 min (Hold 20\% A: 80\% B). 


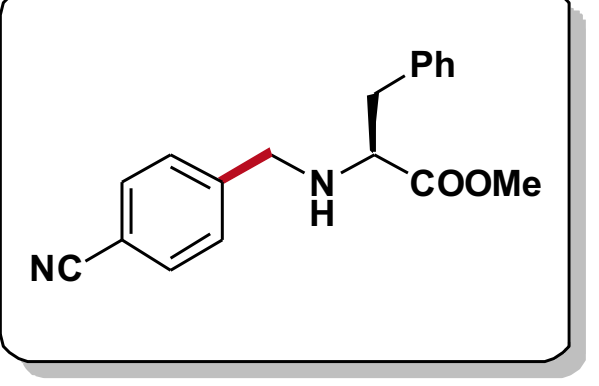

Methyl (4-Cyanobenzyl)-L-phenylalaninate (2a, $87.8 \mathrm{mg}, 99 \%)$ was prepared following General Procedure C. This product was obtained as a pale-yellow oil. ${ }^{1} \mathrm{H}$ NMR $\left(500 \mathrm{MHz}, \mathrm{CDCl}_{3}\right) \delta 7.52$ (d, $J=8.2 \mathrm{~Hz}, 2 \mathrm{H}), 7.31-7.21(\mathrm{~m}, 5 \mathrm{H}), 7.16(\mathrm{~d}, J=8.5 \mathrm{~Hz}, 2 \mathrm{H}), 3.89$ (d, $J=14.4 \mathrm{~Hz}, 1 \mathrm{H}), 3.68(\mathrm{~s}, 3 \mathrm{H}), 3.65(\mathrm{~d}, J=14.4 \mathrm{~Hz}, 1 \mathrm{H}), 3.45$ (t, $J=6.2 \mathrm{~Hz}, 1 \mathrm{H}), 3.00(\mathrm{dd}, J=13.5,5.9 \mathrm{~Hz}, 1 \mathrm{H}), 2.91(\mathrm{dd}, J=13.5,7.7 \mathrm{~Hz}, 1 \mathrm{H}), 1.89(\mathrm{br}, 1 \mathrm{H}) .{ }^{13} \mathrm{C}$ NMR $\left(126 \mathrm{MHz}, \mathrm{CDCl}_{3}\right) \delta 174.9,145.5,137.4,132.2,129.4,128.7,128.6,127.0,119.1,110.9,62.2$, 51.9, 51.6, 39.9. FT-IR ( $\mathrm{cm}^{-1}$, neat, ATR): 3342, 3029, 2950, 2227, 1733, 1608, 1495, 1455, 1414, 1336, 1274, 1200, 1172, 1133, 1019, 994, 822, 750, 726, 701, 548. HRMS (ESI-TOF) calcd for $\left(\mathrm{C}_{18} \mathrm{H}_{19} \mathrm{~N}_{2} \mathrm{O}_{2}\right)$ $[\mathrm{M}+\mathrm{H}]^{+}$294.1447, found 294.1466.

Chiral Supercritical Fluid Chromatography (SFC): $\left(\mathrm{OJ}-3\right.$, methanol $/ \mathrm{CO}_{2}=1 / 99$, flow rate $=2.5 \mathrm{~mL} / \mathrm{min}$, $\mathrm{I}=215 \mathrm{~nm}$ ) $\mathrm{t}_{\mathrm{R}}=4.9 \mathrm{~min}$ (major), $4.3 \mathrm{~min}$ (minor).

\section{Racemic}

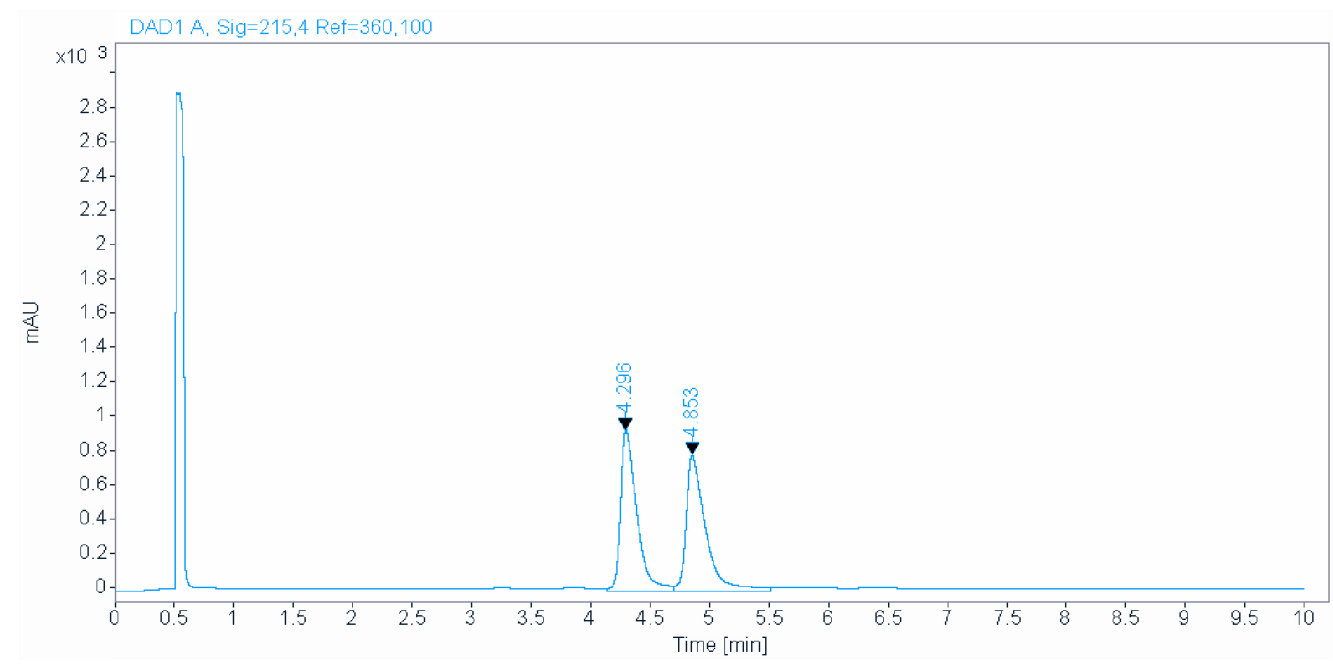

\begin{tabular}{crrrr}
\multicolumn{1}{l}{ Signal: } & \multicolumn{2}{c}{ DAD1 A, Sig $=215,4$ Ref $=360,100$} & & \\
RT [min] Type & Width [min] & Area & Height & Area\% Name \\
4.296 BV & 0.1399 & 8950.4951 & 941.3138 & 49.5543 \\
$4.853 \mathrm{VV}$ & 0.1604 & 9111.5068 & 797.1005 & 50.4457 \\
& Sum & 18062.0020 & &
\end{tabular}




\section{Product}

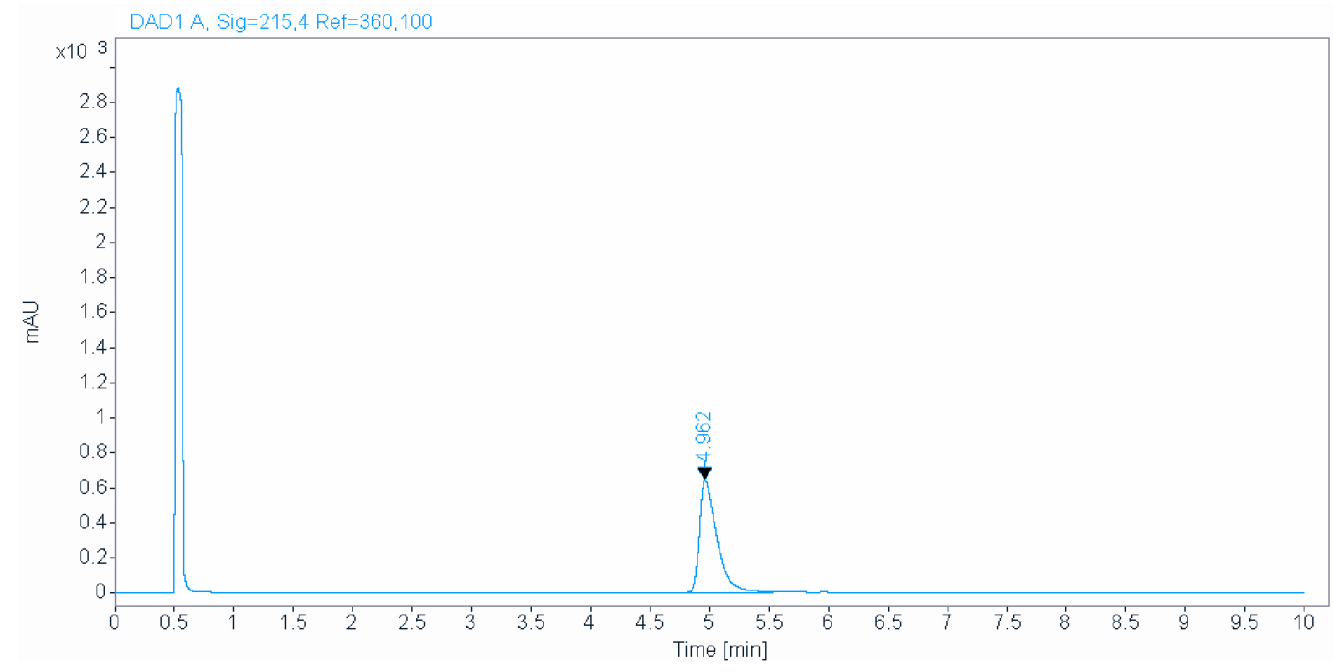

\begin{tabular}{crrrr} 
Signal: & \multicolumn{5}{c}{ DAD1 A, Sig $=215,4$ Ref $=360,100$} & & \\
RT [min] Type & Width [min] & Area & Height & Area\% Name \\
4.962 VV & 0.1527 & 6658.9624 & 643.4202 & 100.0000 \\
& Sum & 6658.9624 & &
\end{tabular}

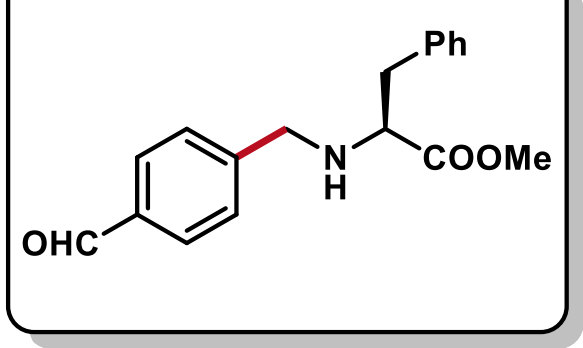

Methyl (4-Formylbenzyl)-L-phenylalaninate (2b, $80.5 \mathrm{mg}$, 90\%) was prepared following General Procedure C. This product was obtained as a pale-yellow oil. ${ }^{1} \mathrm{H}$ NMR $(500 \mathrm{MHz}$, $\left.\mathrm{CDCl}_{3}\right) \delta 9.96(\mathrm{~s}, 1 \mathrm{H}), 7.77(\mathrm{~d}, J=8.0 \mathrm{~Hz}, 2 \mathrm{H}), 7.35(\mathrm{~d}, J=$ $7.8 \mathrm{~Hz}, 2 \mathrm{H}), 7.33-7.21(\mathrm{~m}, 3 \mathrm{H}), 7.17(\mathrm{~d}, J=6.6 \mathrm{~Hz}, 2 \mathrm{H})$, $3.91(\mathrm{~d}, J=14.3 \mathrm{~Hz}, 1 \mathrm{H}), 3.71(\mathrm{~d}, J=14.6 \mathrm{~Hz}, 1 \mathrm{H}), 3.67(\mathrm{~s}, 3 \mathrm{H}), 3.50(\mathrm{~d}, J=6.8 \mathrm{~Hz}, 1 \mathrm{H}), 3.00$ $(\mathrm{dd}, J=13.6,6.1 \mathrm{~Hz}, 1 \mathrm{H}), 2.93(\mathrm{dd}, J=13.6,7.5 \mathrm{~Hz}, 1 \mathrm{H}), 2.08(\mathrm{br}, 1 \mathrm{H}) .{ }^{13} \mathrm{C}$ NMR $(126 \mathrm{MHz}$, $\left.\mathrm{CDCl}_{3}\right) \delta 192.1,175.0,147.1,137.4,135.6,130.0,129.4,128.6,128.6,126.9,62.2,51.9,51.8$ 39.9. FT-IR (cm ${ }^{-1}$, neat, ATR): $3338,2950,1732,1695,1606,1578,1495,1455,1388,1336$, 1304, 1276, 1208, 1167, 1132, 1016, 993, 822, 785, 749, 701. HRMS (ESI-TOF) calcd for $\left(\mathrm{C}_{18} \mathrm{H}_{20} \mathrm{NO}_{3}\right)[\mathrm{M}+\mathrm{H}]^{+}$298.1443, found 298.1443. 


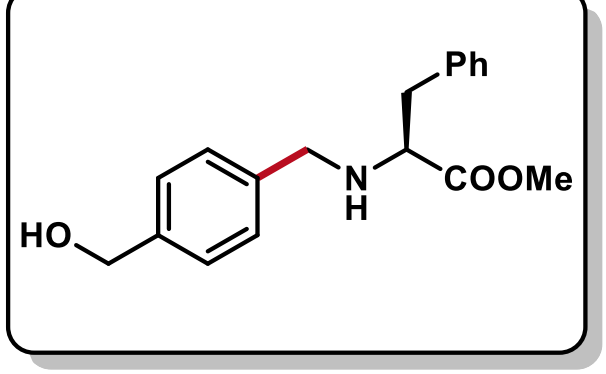

Methyl (4-(Hydroxymethyl)benzyl)-L-phenylalaninate (2c, $65.3 \mathrm{mg}, 73 \%$ ) was prepared following General Procedure C. This product was obtained as a pale-yellow solid $(\mathrm{mp}=45-$ $\left.46{ }^{\circ} \mathrm{C}\right) .{ }^{1} \mathrm{H}$ NMR $\left(600 \mathrm{MHz}, \mathrm{CDCl}_{3}\right) \delta 7.32-7.26(\mathrm{~m}, 4 \mathrm{H}), 7.25-$ $7.22(\mathrm{~m}, 1 \mathrm{H}), 7.21(\mathrm{~d}, J=8.2 \mathrm{~Hz}, 2 \mathrm{H}), 7.16(\mathrm{~d}, J=6.9 \mathrm{~Hz}, 2 \mathrm{H})$, $4.66(\mathrm{~s}, 2 \mathrm{H}), 3.81(\mathrm{~d}, J=13.3 \mathrm{~Hz}, 1 \mathrm{H}), 3.65(\mathrm{~s}, 3 \mathrm{H}), 3.68-3.60(\mathrm{~m}, 1 \mathrm{H}), 3.53(\mathrm{t}, J=6.9 \mathrm{~Hz}, 1 \mathrm{H}), 3.01-$ $2.91(\mathrm{~m}, 2 \mathrm{H}), 1.27(\mathrm{br}, 1 \mathrm{H}) ._{-}{ }^{13} \mathrm{C}$ NMR $\left(151 \mathrm{MHz}, \mathrm{CDCl}_{3}\right) \delta$ 175.0, 139.7, 139.1, 137.3, 129.2, 128.4, 128.4, 127.1, 126.7, 65.2, 62.0, 51.7, 51.7, 39.7. FT-IR ( $\mathrm{cm}^{-1}$, neat, ATR): 3324, 3028, 2950, 2855, 1733, 1604, 1496, 1455, 1365, 1201, 1173, 1127, 1017, 805, 747, 701. HRMS (ESI-TOF) calcd for $\left(\mathrm{C}_{18} \mathrm{H}_{22} \mathrm{NO}_{3}\right)$ $[\mathrm{M}+\mathrm{H}]^{+} 300.1600$, found 300.1608 .

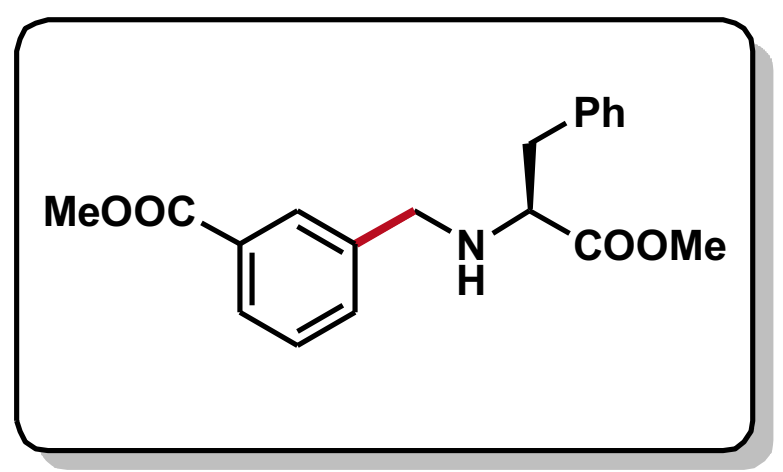

Methyl (S)-3-(((1-Methoxy-1-oxo-3-phenylpropan-2yl) amino)methyl)benzoate (2d, $97.6 \mathrm{mg}, 99 \%)$ was prepared following General Procedure C. This product was obtained as a pale-yellow oil. ${ }^{1} \mathrm{H}$ NMR $(500 \mathrm{MHz}$, $\left.\mathrm{CDCl}_{3}\right) \delta 7.91(\mathrm{~d}, J=11.8 \mathrm{~Hz}, 2 \mathrm{H}), 7.42(\mathrm{~d}, J=7.7 \mathrm{~Hz}$, 1H), $7.34(\mathrm{t}, J=7.6 \mathrm{~Hz}, 1 \mathrm{H}), 7.28(\mathrm{t}, J=7.2 \mathrm{~Hz}, 2 \mathrm{H}), 7.22$ (t, $J=7.3 \mathrm{~Hz}, 1 \mathrm{H}), 7.16(\mathrm{~d}, J=8.6 \mathrm{~Hz}, 2 \mathrm{H}), 3.90(\mathrm{~s}, 3 \mathrm{H}), 3.86(\mathrm{~d}, J=13.5 \mathrm{~Hz}, 1 \mathrm{H}), 3.68(\mathrm{~d}, J=12.5 \mathrm{~Hz}$, 1H), $3.66(\mathrm{~s}, 3 \mathrm{H}), 3.52(\mathrm{t}, J=6.9 \mathrm{~Hz}, 1 \mathrm{H}), 3.03-2.91(\mathrm{~m}, 2 \mathrm{H}), 1.94(\mathrm{br}, 1 \mathrm{H}) .{ }^{13} \mathrm{C} \mathrm{NMR}\left(126 \mathrm{MHz}, \mathrm{CDCl}_{3}\right)$ $\delta 175.0,167.1,140.1,137.3,132.7,130.3,129.3,129.3,128.5,128.4,128.4,126.8,62.1,52.1,51.7$, 51.7, 39.8. FT-IR ( $\mathrm{cm}^{-1}$, neat, ATR): 3332, 3029, 2951, 2845, 1721, 1604, 1590, 1495, 1434, 1284, 1200, 1133, 1107. HRMS (ESI-TOF) calcd for $\left(\mathrm{C}_{19} \mathrm{H}_{22} \mathrm{NO}_{4}\right)[\mathrm{M}+\mathrm{H}]^{+} 328.1549$, found 328.1539. 


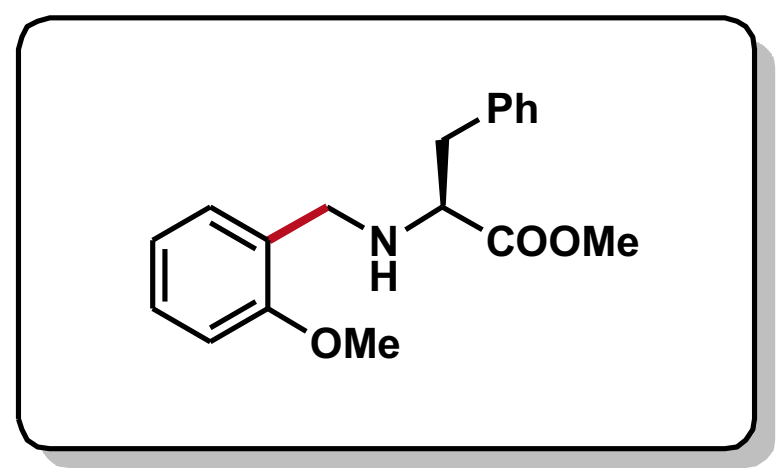

Methyl (2-Methoxybenzyl)-L-phenylalaninate (2e, $70.0 \mathrm{mg}, 78 \%$ ) was prepared following General Procedure C. This product was obtained as a paleyellow oil. ${ }^{1} \mathrm{H}$ NMR $\left(600 \mathrm{MHz}, \mathrm{CDCl}_{3}\right) \delta 7.32-7.25(\mathrm{~m}$, $2 \mathrm{H}), 7.24-7.19(\mathrm{~m}, 2 \mathrm{H}), 7.17-7.12(\mathrm{~m}, 3 \mathrm{H}), 6.87(\mathrm{td}$, $J=7.4,1.1 \mathrm{~Hz}, 1 \mathrm{H}), 6.78(\mathrm{dd}, J=8.2,1.1 \mathrm{~Hz}, 1 \mathrm{H}), 3.81$ (d, J = 13.6 Hz, 1H), 3.70 (d, J = $10.7 \mathrm{~Hz}, 1 \mathrm{H}), 3.68(\mathrm{~s}, 3 \mathrm{H}), 3.61(\mathrm{~s}, 3 \mathrm{H}), 3.53(\mathrm{dd}, J=7.8,6.3 \mathrm{~Hz}, 1 \mathrm{H}$ ), 3.00 (dd, $J=13.5,6.3 \mathrm{~Hz}, 1 \mathrm{H}), 2.93(\mathrm{dd}, J=13.5,7.8 \mathrm{~Hz}, 1 \mathrm{H}), 2.12(\mathrm{br}, 1 \mathrm{H}) .{ }^{13} \mathrm{C} \mathrm{NMR}\left(151 \mathrm{MHz}, \mathrm{CDCl}_{3}\right)$ $\delta 175.02,157.71,137.56,129.86,129.29,128.51,128.42,127.65,126.73,120.41,110.17,62.27,55.18$, 51.74, 47.79, 39.77. FT-IR (cm ${ }^{-1}$, neat, ATR): 2429, 2837, 1733, 1602, 1588, 1493, 1463, 1455, 1438 , 1289, 1241, 1198, 1171, 1115, 1048, 1029, 992, 836, 753, 700. HRMS (ESI-TOF) calcd for $\left(\mathrm{C}_{18} \mathrm{H}_{22} \mathrm{NO}_{3}\right)$ $[\mathrm{M}+\mathrm{H}]^{+} 300.1601$, found 300.1600 .

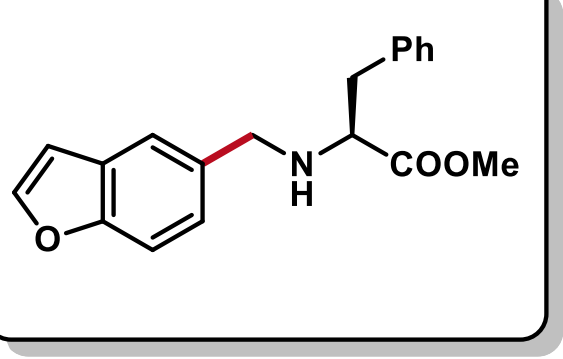

Methyl (Benzofuran-5-ylmethyl)-L-phenylalaninate (2f, 55.0 mg, 59\%) was prepared following General Procedure C. This product was obtained as a pale-yellow oil. ${ }^{1} \mathrm{H}$ NMR $(600 \mathrm{MHz}$, $\left.\mathrm{CDCl}_{3}\right) \delta 7.60(\mathrm{~d}, J=2.2 \mathrm{~Hz}, 1 \mathrm{H}), 7.41(\mathrm{~d}, J=1.0 \mathrm{~Hz}, 1 \mathrm{H}), 7.40(\mathrm{~d}$, $J=8.6 \mathrm{~Hz}, 1 \mathrm{H}), 7.34-7.27(\mathrm{~m}, 2 \mathrm{H}), 7.25-7.21(\mathrm{~m}, 1 \mathrm{H}), 7.20-$ $7.12(\mathrm{~m}, 3 \mathrm{H}), 6.70(\mathrm{dd}, J=2.2,0.9 \mathrm{~Hz}, 1 \mathrm{H}), 3.90(\mathrm{~d}, J=13.0 \mathrm{~Hz}, 1 \mathrm{H}), 3.72(\mathrm{~d}, J=13.0 \mathrm{~Hz}, 1 \mathrm{H}), 3.66(\mathrm{~s}$, $3 \mathrm{H}), 3.56(\mathrm{dd}, J=7.4,6.5 \mathrm{~Hz}, 1 \mathrm{H}), 3.02-2.90(\mathrm{~m}, 2 \mathrm{H}), 1.77(\mathrm{br}, 1 \mathrm{H}) .{ }^{13} \mathrm{C}$ NMR $\left(151 \mathrm{MHz}, \mathrm{CDCl}_{3}\right) \delta$ $175.3,153.0,145.3,137.5,134.2,129.4,128.5,127.6,126.8,124.8,120.7,111.2,106.7,62.1,52.1$, 51.8, 39.9. FT-IR ( $\mathrm{cm}^{-1}$, neat, ATR) 3323, 3022, 2951, 1732, 1495, 1454, 1444, 1333, 1263, 1226, 1197 , 1170, 1128, 1109, 1030, 994, 883, 814, 794, 767, 748, 736, 699. HRMS (ESI-TOF) calcd for $\left(\mathrm{C}_{19} \mathrm{H}_{20} \mathrm{NO}_{3}\right)$ $[\mathrm{M}+\mathrm{H}]^{+} 310.1443$, found 310.1432 . 


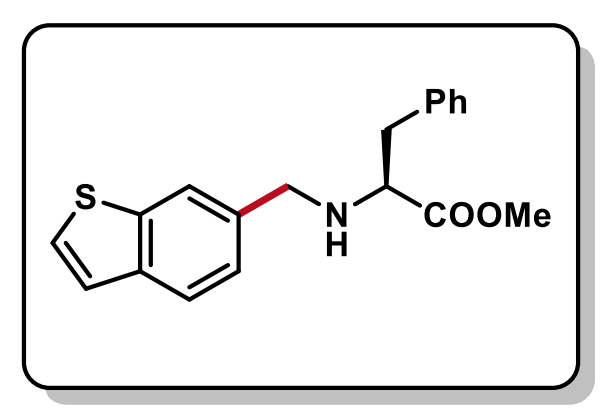

Methyl (Benzo[b]thiophen-6-ylmethyl)-L-phenylalaninate $(2 \mathrm{~g}$, $89.7 \mathrm{mg}, 92 \%$ ) was prepared following General Procedure C. This product was obtained as a pale-yellow oil. ${ }^{1} \mathbf{H}$ NMR $(600 \mathrm{MHz}$, $\left.\mathrm{CDCl}_{3}\right) \delta 7.73(\mathrm{~d}, J=8.1 \mathrm{~Hz}, 1 \mathrm{H}), 7.69(\mathrm{~s}, 1 \mathrm{H}), 7.40(\mathrm{~d}, J=5.4 \mathrm{~Hz}$, $1 \mathrm{H}), 7.34-7.29(\mathrm{~m}, 3 \mathrm{H}), 7.29-7.25(\mathrm{~m}, 1 \mathrm{H}), 7.23(\mathrm{dd}, J=8.1$, $1.5 \mathrm{~Hz}, 1 \mathrm{H}), 7.21-7.17(\mathrm{~m}, 2 \mathrm{H}), 3.97(\mathrm{~d}, J=13.5 \mathrm{~Hz}, 1 \mathrm{H}), 3.78(\mathrm{~d}, J=13.4 \mathrm{~Hz}, 1 \mathrm{H}), 3.68(\mathrm{~s}, 3 \mathrm{H}), 3.59$ (dd, $J=7.5,6.3 \mathrm{~Hz}, 1 \mathrm{H}$ ), 3.02 (dd, $J=13.5,6.3 \mathrm{~Hz}, 1 \mathrm{H}), 2.97$ (dd, $J=13.5,7.5 \mathrm{~Hz}, 1 \mathrm{H}), 1.93$ (br, 1H). ${ }^{13} \mathrm{C}$ NMR $\left(151 \mathrm{MHz}, \mathrm{CDCl}_{3}\right) \delta$ 175.2, 140.0, 138.8, 137.4, 136.0, 129.3, 128.5, 126.8, 126.2, 124.9, 123.6, 123.5, 121.7, 62.0, 52.0, 51.8, 39.9. FT-IR (cm ${ }^{-1}$, neat, ATR) 3346, 3028, 2949, 1732, 1603, 1495, 1455, 1434, 1398, 1342, 1266, 1198, 1171, 1128, 1082, 993, 887, 817, 787, 751, 699. HRMS (ESI-TOF) calcd for $\left(\mathrm{C}_{19} \mathrm{H}_{20} \mathrm{NO}_{2} \mathrm{~S}\right)[\mathrm{M}+\mathrm{H}]^{+} 326.1215$, found 326.1201.

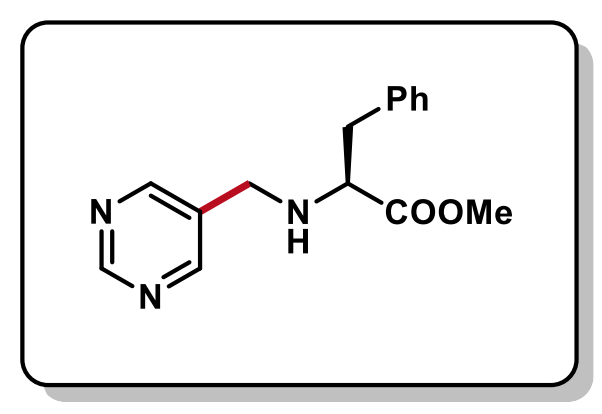

Methyl (Pyrimidin-5-ylmethyl)-L-phenylalaninate (2h, $65.6 \mathrm{mg}$, 81\%) was prepared following General Procedure C with the following modification: The $\mathrm{SiO}_{2}$ column chromatography utilized a gradient elution [flow rate: $30 \mathrm{~mL} / \mathrm{min}, \mathrm{CH}_{2} \mathrm{Cl}_{2}$ (Solvent $\mathrm{A}$ ), $\mathrm{MeOH}$ (Solvent B)]: $1 \min (100 \% A), 4 \min (100 \% A$ to $95 \% A: 5 \% B), 4$ $\min (95 \%$ A: $5 \%$ B to $93 \%$ A: $7 \%$ B), $3 \min (93 \%$ A: $7 \%$ B to $90 \%$ A: 10\% B), 2 min (Hold $90 \%$ A: 10\% B). This product was obtained as a pale-yellow oil. ${ }^{1} \mathrm{H}$ NMR $\left(600 \mathrm{MHz}, \mathrm{CDCl}_{3}\right) \delta 9.07(\mathrm{~s}, 1 \mathrm{H}), 8.55(\mathrm{~s}$, 2H), $7.35-7.24(\mathrm{~m}, 2 \mathrm{H}), 7.24-7.19(\mathrm{~m}, 1 \mathrm{H}), 7.14-7.10(\mathrm{~m}, 2 \mathrm{H}), 3.83(\mathrm{~d}, J=14.1 \mathrm{~Hz}, 1 \mathrm{H}), 3.67(\mathrm{~s}$, 3H), $3.57(\mathrm{~d}, J=14.1 \mathrm{~Hz}, 1 \mathrm{H}), 3.47(\mathrm{dd}, J=7.6,5.9 \mathrm{~Hz}, 1 \mathrm{H}), 2.99(\mathrm{dd}, J=13.6,5.9 \mathrm{~Hz}, 1 \mathrm{H}), 2.89(\mathrm{dd}, J$ $=13.6,7.6 \mathrm{~Hz}, 1 \mathrm{H}), 1.94(\mathrm{br}, 1 \mathrm{H}) .{ }^{13} \mathrm{C}$ NMR $\left(151 \mathrm{MHz}, \mathrm{CDCl}_{3}\right) \delta 174.6,157.8,156.7,137.0,132.9,129.2$, 128.5, 127.0, 62.1 , 51.9, 47.1, 39.8. FT-IR (cm ${ }^{-1}$, neat, ATR) 3322, 3028, 2951, 1733, 1584, 1563, 1496, 1455, 1438, 1407, 1376, 1348, 1271, 1201, 1172, 1134, 1002, 747, 728, 701, 634. HRMS (ESI-TOF) calcd for $\left(\mathrm{C}_{15} \mathrm{H}_{18} \mathrm{~N}_{3} \mathrm{O}_{2}\right)[\mathrm{M}+\mathrm{H}]^{+} 272.1399$, found 272.1409. 


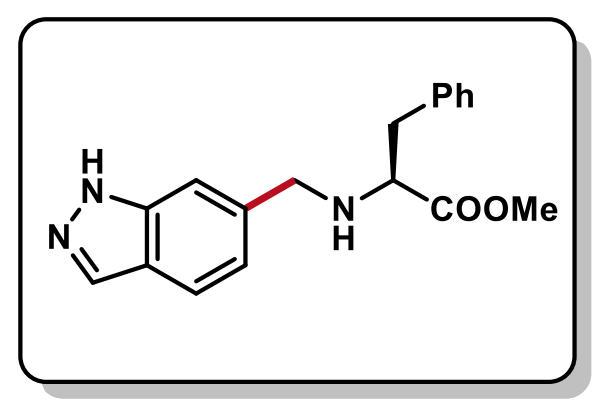

Methyl ((1H-Indazol-6-yl)methyl)-L-phenylalaninate (2i, 67.5 mg, 73\%) was prepared following General Procedure C with the following modification: The $\mathrm{SiO}_{2}$ column chromatography utilized a gradient elution [flow rate: $30 \mathrm{~mL} / \mathrm{min}, \mathrm{CH}_{2} \mathrm{Cl}_{2}$ (Solvent $\mathrm{A}$ ), $\mathrm{MeOH}$ (Solvent B)]: $1 \min (100 \% A), 4 \min (100 \% A$ to $95 \% A: 5 \% B), 4$ $\min (95 \%$ A: $5 \%$ B to $93 \%$ A: $7 \%$ B), $3 \min (93 \% A: 7 \%$ B to $90 \% A: 10 \%$ B), $2 \min ($ Hold $90 \%$ A: $10 \%$ B). This product was obtained as a white solid $\left(\mathrm{mp}=130-131^{\circ} \mathrm{C}\right) .{ }^{1} \mathbf{H} \mathbf{N M R}\left(600 \mathrm{MHz}, \mathrm{CDCl}_{3}\right) \delta 9.99$ (br, 1H), $8.02(\mathrm{~s}, 1 \mathrm{H}), 7.63(\mathrm{~d}, J=8.3 \mathrm{~Hz}, 1 \mathrm{H}), 7.32-7.27(\mathrm{~m}, 2 \mathrm{H}), 7.27-7.24(\mathrm{~m}, 1 \mathrm{H}), 7.21(\mathrm{~s}, 1 \mathrm{H})$, $7.18(\mathrm{~d}, J=5.1 \mathrm{~Hz}, 2 \mathrm{H}), 7.01(\mathrm{~d}, J=8.3 \mathrm{~Hz}, 1 \mathrm{H}), 3.97(\mathrm{~d}, J=13.8 \mathrm{~Hz}, 1 \mathrm{H}), 3.77(\mathrm{~d}, J=13.1 \mathrm{~Hz}, 1 \mathrm{H})$, 3.67 (s, 3H), 3.54 (dd, $J=7.8,6.0 \mathrm{~Hz}, 1 \mathrm{H}), 3.02(\mathrm{dd}, J=13.5,6.0 \mathrm{~Hz}, 1 \mathrm{H}), 2.93(\mathrm{dd}, J=13.5,7.8 \mathrm{~Hz}$, 1H), 1.95 (br, 1H). ${ }^{13} \mathrm{C}$ NMR (151 MHz, CDCl 3$) \delta 175.3,140.5,139.1,137.7,135.0,129.5,128.6,126.8$, 122.6, 121.9, 120.7, 108.5, 62.1, 52.1, 51.9, 40.0. FT-IR (cm ${ }^{-1}$, neat, ATR) 3318, 3190, 2950, 1735, 1454, 1202, 943, 701. HRMS (ESI-TOF) calcd for $\left(\mathrm{C}_{18} \mathrm{H}_{20} \mathrm{~N}_{3} \mathrm{O}_{2}\right)[\mathrm{M}+\mathrm{H}]^{+} 310.1556$, found 310.1561.

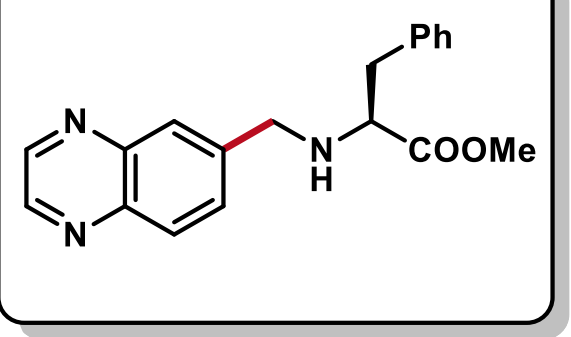

Methyl (Quinoxalin-6-ylmethyl)-L-phenylalaninate (2j, $81.0 \mathrm{mg}$, $84 \%$ ) was prepared following General Procedure C. This product was obtained as a pale-yellow oil. ${ }^{1} \mathrm{H}$ NMR $\left(600 \mathrm{MHz}, \mathrm{CDCl}_{3}\right) \delta$ $8.79(\mathrm{~d}, J=7.8 \mathrm{~Hz}, 2 \mathrm{H}), 7.98(\mathrm{~d}, J=8.6 \mathrm{~Hz}, 1 \mathrm{H}), 7.94(\mathrm{~s}, 1 \mathrm{H}), 7.64$ (dd, $J=8.7,1.9 \mathrm{~Hz}, 1 \mathrm{H}), 7.25(\mathrm{t}, J=8.4 \mathrm{~Hz}, 2 \mathrm{H}), 7.21(\mathrm{t}, J=7.3$ $\mathrm{Hz}, 1 \mathrm{H}), 7.15(\mathrm{~d}, J=6.9 \mathrm{~Hz}, 2 \mathrm{H}), 4.06(\mathrm{~d}, J=14.1 \mathrm{~Hz}, 1 \mathrm{H}), 3.84(\mathrm{~d}, J=14.1 \mathrm{~Hz}, 1 \mathrm{H}), 3.66(\mathrm{~s}, 3 \mathrm{H}), 3.55$ $(\mathrm{t}, J=6.8 \mathrm{~Hz}, 1 \mathrm{H}), 3.04-2.93(\mathrm{~m}, 2 \mathrm{H}), 2.00(\mathrm{br}, 1 \mathrm{H}) .{ }^{13} \mathrm{C}$ NMR $\left(151 \mathrm{MHz}, \mathrm{CDCl}_{3}\right) \delta 174.9,145.0,144.6$, 143.0, 142.5, 142.4, 137.2, 130.7, 129.4, 129.3, 128.5, 127.8, 126.8, 62.0, 51.8, 51.7, 39.8. FT-IR $\left(\mathrm{cm}^{-}\right.$ ${ }^{1}$, neat, ATR) 3318, 3028, 2950, 2850, 1733, 1623, 1497, 1454, 1367, 1200, 1172, 1131, 1025, 997, 957 , 895, 868, 823, 745, 701. HRMS (ESI-TOF) calcd for $\left(\mathrm{C}_{19} \mathrm{H}_{20} \mathrm{~N}_{3} \mathrm{O}_{2}\right)[\mathrm{M}+\mathrm{H}]^{+} 322.1556$, found 322.1569. 


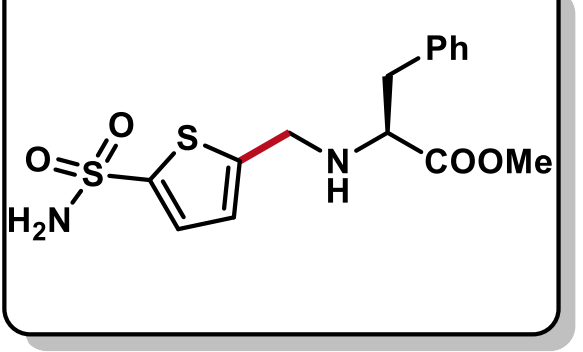

\section{Methyl ((5-Sulfamoylthiophen-2-yl)methyl)-L-phenylalaninate} (2k, 45.7 mg, 43\%) was prepared following General Procedure C.

This product was obtained as a pale-yellow oil. ${ }^{1} \mathrm{H}$ NMR $(600 \mathrm{MHz}$, $\left.\mathrm{CDCl}_{3}\right) \delta 7.46(\mathrm{~d}, J=3.8 \mathrm{~Hz}, 1 \mathrm{H}), 7.29(\mathrm{t}, J=7.3 \mathrm{~Hz}, 2 \mathrm{H}), 7.24(\mathrm{t}$, $J=7.3 \mathrm{~Hz}, 1 \mathrm{H}), 7.16(\mathrm{~d}, J=6.9 \mathrm{~Hz}, 2 \mathrm{H}), 6.75(\mathrm{~d}, J=3.7 \mathrm{~Hz}, 1 \mathrm{H})$, $5.09(\mathrm{br}, 2 \mathrm{H}), 4.05(\mathrm{~d}, J=16.1 \mathrm{~Hz}, 1 \mathrm{H}), 3.78(\mathrm{~d}, J=14.9 \mathrm{~Hz}, 1 \mathrm{H}), 3.69(\mathrm{~s}, 3 \mathrm{H}), 3.56(\mathrm{~d}, J=6.3 \mathrm{~Hz}, 1 \mathrm{H})$, $3.00(\mathrm{dd}, J=13.5,6.0 \mathrm{~Hz}, 1 \mathrm{H}), 2.94(\mathrm{dd}, J=13.5,7.1 \mathrm{~Hz}, 1 \mathrm{H}), 1.99(\mathrm{br}, 1 \mathrm{H}) .{ }^{13} \mathrm{C} \mathrm{NMR}\left(151 \mathrm{MHz}, \mathrm{CDCl}_{3}\right)$ $\delta 174.7,152.5,141.4,137.0,131.8,129.5,128.6,127.0,124.2,61.8,52.0,47.0,39.8 . \mathbf{F T}-\mathbf{I R}\left(\mathrm{cm}^{-1}\right.$, neat, ATR) 3329, 3029, 2952, 1732, 1604, 1496, 1439, 1340, 1209, 1158, 1081, 1015, 903, 811, 747, 702, 603, 539. HRMS (ESI-TOF) calcd for $\left(\mathrm{C}_{15} \mathrm{H}_{19} \mathrm{~N}_{2} \mathrm{O}_{4} \mathrm{~S}_{2}\right)[\mathrm{M}+\mathrm{H}]^{+} 355.0786$, found 355.0794 .

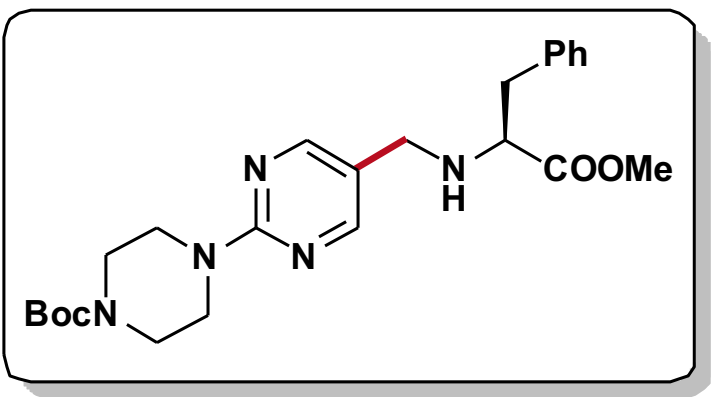

tert-Butyl (S)-4-(5-)((1-Methoxy-1-oxo-3-phenylpropan2-yl)amino)methyl)pyrimidin-2-yl)piperazine-1carboxylate (2I, $127.7 \mathrm{mg}, 93 \%)$ was prepared following General Procedure C. This product was obtained as a white solid $\left(\mathrm{mp}=56-57^{\circ} \mathrm{C}\right) .{ }^{1} \mathrm{H}$ NMR $\left(600 \mathrm{MHz}, \mathrm{CDCl}_{3}\right)$ $\delta 8.18(\mathrm{~s}, 2 \mathrm{H}), 7.30-7.23(\mathrm{~m}, 2 \mathrm{H}), 7.23-7.17(\mathrm{~m}, 1 \mathrm{H}), 7.13(\mathrm{dd}, J=6.8,1.5 \mathrm{~Hz}, 2 \mathrm{H}), 3.77(\mathrm{t}, J=5.3$ $\mathrm{Hz}, 4 \mathrm{H}), 3.65(\mathrm{~s}, 3 \mathrm{H}), 3.62(\mathrm{~d}, J=12.9 \mathrm{~Hz}, 1 \mathrm{H}), 3.50(\mathrm{~d}, J=7.0 \mathrm{~Hz}, 1 \mathrm{H}), 3.48-3.45(\mathrm{~m}, 4 \mathrm{H}), 3.41(\mathrm{~d}, J$ $=13.2 \mathrm{~Hz}, 1 \mathrm{H}), 2.97-2.86(\mathrm{~m}, 2 \mathrm{H}), 1.74(\mathrm{br}, 1 \mathrm{H}), 1.47(\mathrm{~s}, 9 \mathrm{H}) .{ }^{13} \mathrm{C}$ NMR $\left(151 \mathrm{MHz}, \mathrm{CDCl}_{3}\right) \delta 174.9$, 161.4, 158.0, 154.9, 137.1, 129.2, 128.5, 126.9, 120.8, 80.0, 61.8, 51.8, 46.9, 43.8, 39.8, 28.5. FT-IR (cm ${ }^{-1}$, neat, ATR) 3320, 2930, 1736, 1694, 1605, 1544, 1497, 1451, 1417, 1360, 1304, 1278, 1244, 1168, 1127, 1088, 998, 949, 797, 769, 701. HRMS (ESI-TOF) calcd for $\left(\mathrm{C}_{24} \mathrm{H}_{34} \mathrm{~N}_{5} \mathrm{O}_{4}\right)[\mathrm{M}+\mathrm{H}]^{+} 456.2611$, found 456.2620. 


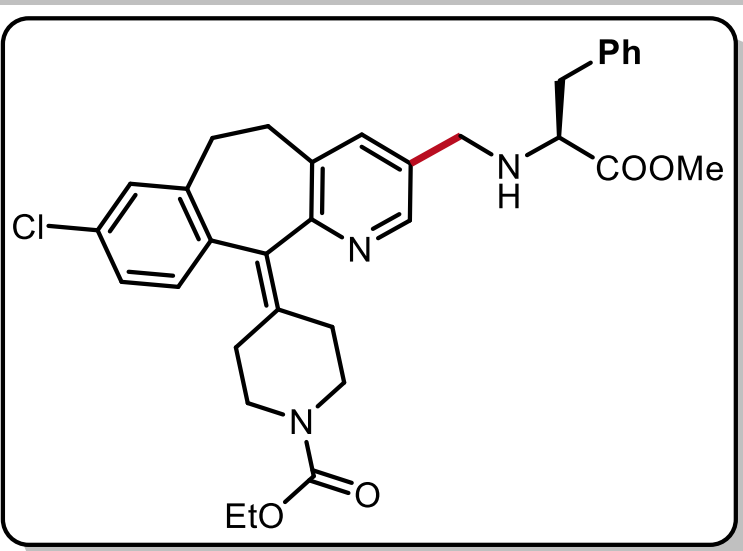

Ethyl (S)-4-(8-Chloro-3-(((1-methoxy-1-oxo-3-phenyl

propan-2-yl)amino)methyl)-5,6-dihydro-11H-benzo

[5,6]cyclohepta[1,2-b]pyridin-11-ylidene) piperidine1-carboxylate $(2 \mathrm{~m}, 108.1 \mathrm{mg}, 63 \%)$ was prepared following General Procedure C. This product was obtained as a pale-yellow oil. ${ }^{1} \mathrm{H}$ NMR $\left(600 \mathrm{MHz}, \mathrm{CDCl}_{3}\right)$ [1:1 mixture of atropisomers] $\delta 8.24-8.19(\mathrm{~s}, 1 \mathrm{H}), 7.28$ $-7.19(\mathrm{~m}, 4 \mathrm{H}), 7.18-7.06(\mathrm{~m}, 5 \mathrm{H}), 4.13(\mathrm{q}, J=7.1,2 \mathrm{H}), 3.84-3.78(\mathrm{~m}, 2 \mathrm{H}), 3.75(\mathrm{t}, J=13.7 \mathrm{~Hz}, 1 \mathrm{H})$, $3.65-3.63(\mathrm{~s}, 3 \mathrm{H}), 3.55(\mathrm{t}, J=11.1 \mathrm{~Hz}, 1 \mathrm{H}), 3.52-3.44(\mathrm{~m}, 1 \mathrm{H}), 3.39-3.31(\mathrm{~m}, 1 \mathrm{H}), 3.29-3.22(\mathrm{~m}$, 1H), $3.18-3.06(\mathrm{~m}, 2 \mathrm{H}), 2.97(\mathrm{ddd}, J=13.6,6.1,2.7 \mathrm{~Hz}, 1 \mathrm{H}), 2.94-2.81(\mathrm{~m}, 1 \mathrm{H}), 2.80-2.69(\mathrm{~m}, 2 \mathrm{H})$, $2.50-2.38(\mathrm{~m}, 1 \mathrm{H}), 2.39-2.24(\mathrm{~m}, 3 \mathrm{H}), 1.84(\mathrm{br}, 1 \mathrm{H}), 1.24(\mathrm{t}, J=6.3 \mathrm{~Hz}, 3 \mathrm{H}) .{ }^{13} \mathrm{C}$ NMR $(151 \mathrm{MHz}$, $\left.\mathrm{CDCl}_{3}\right)[1: 1$ mixture of atropisomers $] \delta 174.9,174.8,155.8,155.5,146.5,146.4,139.6,139.6,137.8$ $137.8,137.5,137.5,137.4,137.3,137.3,137.2,134.0,133.6,133.6,133.0,133.0,132.9,130.5,130.5$, $129.2,128.9,128.4,128.4,126.8,126.2,62.1,62.0,61.3,51.8,51.8,49.0,49.0,44.8,44.8,39.7,39.7$, 31.7, 31.5, 31.4, 30.8, 30.5, 14.7. FT-IR (cm-1, neat, ATR) 3337, 2978, 2917, 2852, 1734, 1693, 1475 , 1432, 1386, 1358, 1325, 1277, 1258, 1222, 1172, 1116, 1088, 1029, 997, 893, 826, 810, 767, 701. HRMS (ESI-TOF) calcd for $\left(\mathrm{C}_{33} \mathrm{H}_{37} \mathrm{CIN}_{3} \mathrm{O}_{4}\right)[\mathrm{M}+\mathrm{H}]^{+} 574.2473$, found 574.2479.

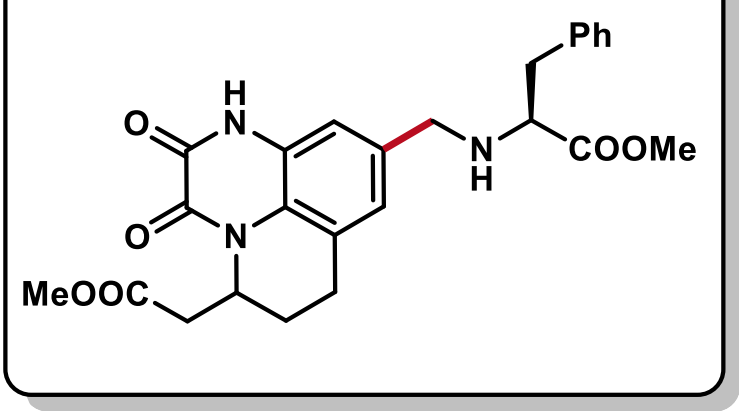

(Solvent A), $\mathrm{MeOH}$ (Solvent B)]: 2 min (100\% A), 6 min (100\% A to 95\% A: 5\% B), 8 min (Hold 95\% A: $5 \% \mathrm{~B})$. This product was obtained as a pale-yellow oil. ${ }^{1} \mathrm{H}$ NMR $\left(600 \mathrm{MHz}, \mathrm{CDCl}_{3}\right)[1: 1$ mixture of diastereomers] $\delta 11.23(\mathrm{br}, 1 \mathrm{H}), 7.33-7.27(\mathrm{~m}, 2 \mathrm{H}), 7.27-7.24(\mathrm{~m}, 1 \mathrm{H}), 7.23-7.16(\mathrm{~m}, 2 \mathrm{H}), 6.92-$
Methyl ((5-(2-Methoxy-2-oxoethyl)-2,3-dioxo-2,3,6,7tetrahydro-1H,5H-pyrido[1,2,3-de]quinoxalin-9yl)methyl)-L-phenylalaninate $(2 \mathrm{n}, 85.4 \mathrm{mg}, 61 \%)$ was prepared following General Procedure C with the following modification: The $\mathrm{SiO}_{2}$ column chromatography utilized a gradient elution [flow rate: $30 \mathrm{~mL} / \mathrm{min}, \mathrm{CH}_{2} \mathrm{Cl}_{2}$ 
$6.89(\mathrm{~m}, 1 \mathrm{H}), 6.85(\mathrm{~s}, 1 \mathrm{H}), 5.40(\mathrm{dtd}, J=9.8,4.9,2.2 \mathrm{~Hz}, 1 \mathrm{H}), 3.81(\mathrm{~d}, J=13.8 \mathrm{~Hz}, 1 \mathrm{H}), 3.72-3.71(\mathrm{~s}$, 3H), $3.70(\mathrm{~s}, 3 \mathrm{H}), 3.58$ (d, $J=13.7 \mathrm{~Hz}, 1 \mathrm{H}), 3.46$ (ddd, $J=7.9,6.0,1.9 \mathrm{~Hz}, 1 \mathrm{H}), 2.99$ (ddd, $J=13.5,5.9$, $1.2 \mathrm{~Hz}, 1 \mathrm{H}), 2.96-2.86(\mathrm{~m}, 2 \mathrm{H}), 2.80(\mathrm{dd}, J=15.2,5.1 \mathrm{~Hz}, 1 \mathrm{H}), 2.77-2.69(\mathrm{~m}, 1 \mathrm{H}), 2.54(\mathrm{dd}, J=15.2$, $9.7 \mathrm{~Hz}, 1 \mathrm{H}), 2.29(\mathrm{ddd}, J=14.2,5.1,2.4 \mathrm{~Hz}, 1 \mathrm{H}), 2.00-1.92(\mathrm{~m}, 1 \mathrm{H}), 1.86(\mathrm{br}, 1 \mathrm{H}) .{ }^{13} \mathrm{C} \mathbf{N M R}(151 \mathrm{MHz}$, $\left.\mathrm{CDCl}_{3}\right)[1: 1$ mixture of diastereomers] $\delta 175.1,170.7,170.7,155.3,154.2,137.7,136.2,129.5,128.5$, $126.8,126.8,124.7,124.7,124.6,124.3,124.3,121.7,114.3,114.3,62.2,52.2,52.0,50.9,47.9,47.9$, 39.9, 35.3, 29.4, 23.3, 21.8, 21.8. FT-IR ( $\mathrm{cm}^{-1}$, neat, ATR) 2950, 1734, 1698, 1602, 1528, 1438, 1388 , 1279, 1199, 1175, 1020, 861, 745, 702. HRMS (ESI-TOF) calcd for $\left(\mathrm{C}_{25} \mathrm{H}_{28} \mathrm{~N}_{3} \mathrm{O}_{6}\right)[\mathrm{M}+\mathrm{H}]^{+} 466.1978$, found 466.1996.

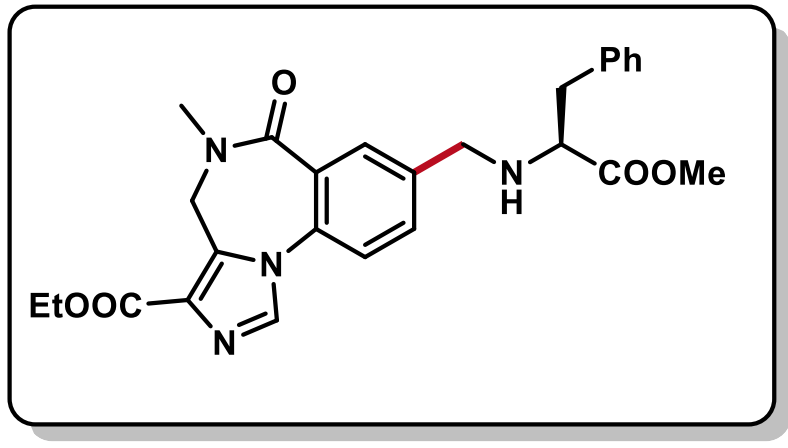

Ethyl (S)-8-(((1-Methoxy-1-oxo-3-phenylpropan-2yl)amino)methyl)-5-methyl-6-oxo-5,6-dihydro-4Hbenzo[f]imidazo[1,5-a][1,4]diazepine-3-carboxylate (2o, $141.2 \mathrm{mg}, 99 \%)$ was prepared following General Procedure $\mathbf{C}$ with the following modification: $\mathrm{The}^{\mathrm{SiO}_{2}}$ column chromatography utilized a gradient elution [flow rate: $30 \mathrm{~mL} / \mathrm{min}, \mathrm{CH}_{2} \mathrm{Cl}_{2}$ (Solvent A), $\mathrm{MeOH}$ (Solvent B)]: $2 \min (100 \% \mathrm{~A}), 4 \min (100 \% \mathrm{~A}$ to $90 \% \mathrm{~A}: 10 \%$ B), $4 \min ($ Hold $90 \%$ A: 10\% B), $2 \min (90 \%$ A: 10\% B to $80 \%$ A: $20 \%$ B), 4 min (Hold 80\% A: $20 \%$ B). This product was obtained as a white foam. ${ }^{1} \mathrm{H}$ NMR $\left(400 \mathrm{MHz}, \mathrm{CDCl}_{3}\right) \delta 7.85(\mathrm{br}, 1 \mathrm{H}), 7.81(\mathrm{~s}, 1 \mathrm{H})$, $7.41(\mathrm{dd}, J=8.3,2.0 \mathrm{~Hz}, 1 \mathrm{H}), 7.26-7.23(\mathrm{~m}, 1 \mathrm{H}), 7.23-7.18(\mathrm{~m}, 2 \mathrm{H}), 7.18-7.13(\mathrm{~m}, 1 \mathrm{H}), 7.12-7.09$ (m, 2H), $5.10(\mathrm{br}, 1 \mathrm{H}), 4.36(\mathrm{~d}, J=4.8 \mathrm{~Hz}, 2 \mathrm{H}), 4.28(\mathrm{br}, 1 \mathrm{H}), 3.86(\mathrm{~d}, J=14.0 \mathrm{~Hz}, 1 \mathrm{H}), 3.65(\mathrm{~d}, J=11.8$ $\mathrm{Hz}, 1 \mathrm{H}), 3.62(\mathrm{~s}, 3 \mathrm{H}), 3.46-3.41(\mathrm{~m}, 1 \mathrm{H}), 3.17(\mathrm{~s}, 3 \mathrm{H}), 3.00-2.80(\mathrm{~m}, 2 \mathrm{H}), 2.04(\mathrm{br}, 1 \mathrm{H}), 1.37(\mathrm{t}, J=$ $7.1 \mathrm{~Hz}, 3 \mathrm{H}) .{ }^{13} \mathrm{C}$ NMR $\left(101 \mathrm{MHz}, \mathrm{CDCl}_{3}\right) \delta 174.6,166.4,162.9,140.7,137.1,135.4,134.9,132.1,131.8$, 130.7, 129.2, 128.7, 128.5, 128.3, 126.6, 121.8, 62.0, 60.8, 51.7, 50.8, 42.3, 39.6, 35.7, 14.3. FT-IR $\left(\mathrm{cm}^{-}\right.$ ${ }^{1}$, neat, ATR) 3450, 3325, 3033, 2975, 2950, 1731, 1702, 1642, 1610, 1572, 1497, 1455, 1391, 1375, $1351,1314,1289,1259,1194,1171,1114,1064,844,762,702$. HRMS (ESI-TOF) calcd for $\left(\mathrm{C}_{26} \mathrm{H}_{29} \mathrm{~N}_{4} \mathrm{O}_{5}\right)$ $[\mathrm{M}+\mathrm{H}]^{+}$477.2138, found 477.2131. 


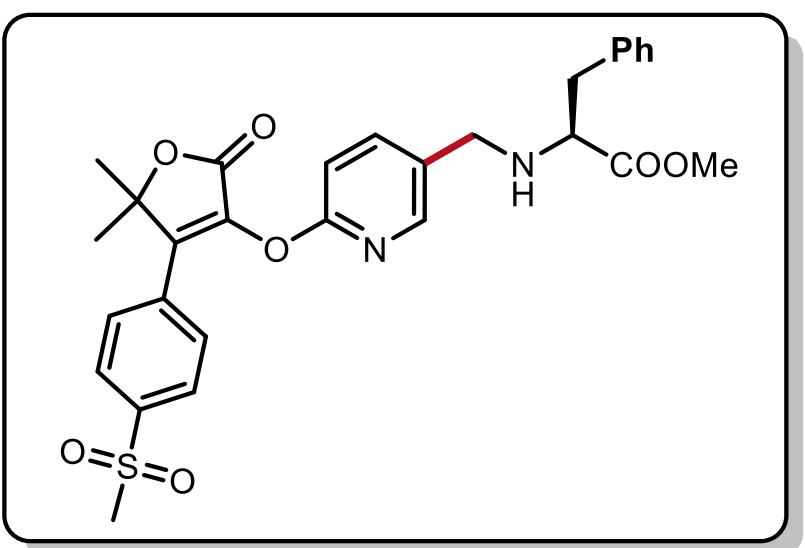

Methyl ((6-)(5,5-Dimethyl-4-(4-(methylsulfonyl) phenyl)-2-oxo-2,5-dihydrofuran-3-yl)oxy)pyridin3-yl)methyl)-L-phenylalaninate (2p, $88.3 \mathrm{mg}, 54 \%)$ was prepared following General Procedure C. This product was obtained as a pale-yellow oil. ${ }^{1} \mathbf{H}$ NMR $\left(600 \mathrm{MHz}, \mathrm{CDCl}_{3}\right) \delta 7.98(\mathrm{~d}, J=8.6 \mathrm{~Hz}, 2 \mathrm{H}), 7.95(\mathrm{~d}$, $J=1.7 \mathrm{~Hz}, 1 \mathrm{H}), 7.77-7.72(\mathrm{~m}, 2 \mathrm{H}), 7.56(\mathrm{dd}, J=8.4$, $2.4 \mathrm{~Hz}, 1 \mathrm{H}), 7.29-7.25(\mathrm{~m}, 2 \mathrm{H}), 7.23-7.20(\mathrm{~m}, 1 \mathrm{H}), 7.15-7.13(\mathrm{~m}, 2 \mathrm{H}), 6.89(\mathrm{dd}, J=8.4,0.7 \mathrm{~Hz}, 1 \mathrm{H})$, $3.75(\mathrm{~d}, J=13.5 \mathrm{~Hz}, 1 \mathrm{H}), 3.66(\mathrm{~s}, 3 \mathrm{H}), 3.55(\mathrm{~d}, J=13.5 \mathrm{~Hz}, 1 \mathrm{H}), 3.48(\mathrm{dd}, J=7.4,6.1 \mathrm{~Hz}, 1 \mathrm{H}), 3.05$ (s, 3H), 2.97 (dd, $J=13.6,6.1 \mathrm{~Hz}, 1 \mathrm{H}), 2.90(\mathrm{dd}, J=13.6,7.4 \mathrm{~Hz}, 1 \mathrm{H}), 1.80(\mathrm{br}, 1 \mathrm{H}), 1.75(\mathrm{~s}, 6 \mathrm{H}) .{ }^{13} \mathrm{C}$ NMR $\left(151 \mathrm{MHz}, \mathrm{CDCl}_{3}\right) \delta 174.9,166.0,160.6,148.5,146.9,141.4,140.1,138.0,137.2,135.1,131.3,129.3$, 129.0, 128.5, 128.0, 126.9, 110.8, 84.4, 62.0, 51.9, 48.7, 44.4, 39.8, 26.5. FT-IR (cm ${ }^{-1}$, neat, ATR) 2976, 2925, 1767, 1733, 1600, 1477, 1398, 1347, 1313, 1232, 1176, 1150, 1095, 1015, 960, 881, 840, 773, 728, 702, 552, 534. HRMS (ESI-TOF) calcd for $\left(\mathrm{C}_{29} \mathrm{H}_{31} \mathrm{~N}_{2} \mathrm{O}_{7} \mathrm{~S}\right)[\mathrm{M}+\mathrm{H}]^{+} 551.1852$, found 551.1849.

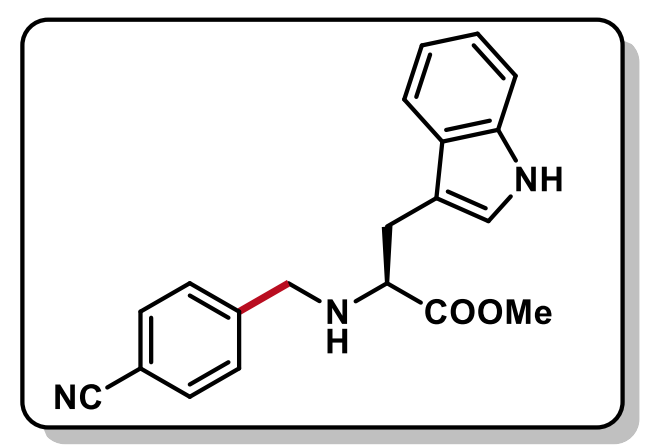

Methyl (4-Cyanobenzyl)-L-tryptophanate (2q, $95.6 \mathrm{mg}, 96 \%)$ was prepared following General Procedure C. This product was obtained as a pale-yellow oil. ${ }^{1} \mathrm{H}$ NMR $\left(500 \mathrm{MHz}, \mathrm{CDCl}_{3}\right) \delta 8.26$ (br, $1 \mathrm{H}), 7.56(\mathrm{~d}, J=8.0 \mathrm{~Hz}, 1 \mathrm{H}), 7.47(\mathrm{~d}, J=8.2 \mathrm{~Hz}, 2 \mathrm{H}), 7.37$ - $7.32(\mathrm{~m}, 1 \mathrm{H}), 7.27(\mathrm{~d}, J=8.0 \mathrm{~Hz}, 2 \mathrm{H}), 7.20$ (ddd, $J=8.0,6.9$, $1.2 \mathrm{~Hz}, 1 \mathrm{H}), 7.10$ (ddd, $J=7.9,6.9,1.0 \mathrm{~Hz}, 1 \mathrm{H}), 7.02(\mathrm{~d}, J=2.4$ $\mathrm{Hz}, 1 \mathrm{H}), 3.89(\mathrm{~d}, J=14.4 \mathrm{~Hz}, 1 \mathrm{H}), 3.68(\mathrm{~s}, 3 \mathrm{H}), 3.67(\mathrm{~d}, J=14.4 \mathrm{~Hz}, 1 \mathrm{H}), 3.61(\mathrm{dd}, J=7.3,5.6 \mathrm{~Hz}, 1 \mathrm{H})$, $3.23(\mathrm{dd}, J=14.5,5.6 \mathrm{~Hz}, 1 \mathrm{H}), 3.12(\mathrm{dd}, J=14.5,7.3 \mathrm{~Hz}, 1 \mathrm{H}), 2.03(\mathrm{br}, 1 \mathrm{H}) .{ }^{13} \mathrm{C} \mathrm{NMR}\left(126 \mathrm{MHz}, \mathrm{CDCl}_{3}\right)$ $\delta 175.2,145.5,136.3,132.1,128.6,127.5,123.0,122.2,119.5,119.1,118.8,111.3,111.1,110.6,61.1$, 51.9, 51.6, 29.4. FT-IR ( $\mathrm{cm}^{-1}$, neat, ATR) 3402, 2950, 2924, 2227, 1731, 1608, 1505, 1457, 1434, 1342, 
$1280,1203,1174,1150,1133,1096,1010,819,743,548$. HRMS (ESI-TOF) calcd for $\left(\mathrm{C}_{20} \mathrm{H}_{20} \mathrm{~N}_{3} \mathrm{O}_{2}\right)$ $[\mathrm{M}+\mathrm{H}]^{+} 334.1556$, found 334.1545 .

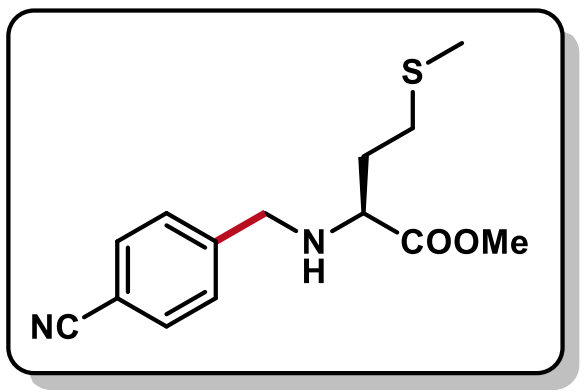

Methyl (4-Cyanobenzyl)-L-methioninate (2r, $82.8 \mathrm{mg}, 99 \%)$ was prepared following General Procedure C. This product was obtained as a pale-yellow oil. ${ }^{1} \mathrm{H}$ NMR $\left(500 \mathrm{MHz}, \mathrm{CDCl}_{3}\right) \delta 7.57(\mathrm{~d}$, $J=8.4 \mathrm{~Hz}, 2 \mathrm{H}), 7.42(\mathrm{~d}, J=8.1 \mathrm{~Hz}, 2 \mathrm{H}), 3.89(\mathrm{~d}, J=14.1 \mathrm{~Hz}, 1 \mathrm{H})$, $3.71(\mathrm{~s}, 3 \mathrm{H}), 3.64(\mathrm{~d}, J=14.1 \mathrm{~Hz}, 1 \mathrm{H}), 3.36(\mathrm{dd}, J=8.4,5.0 \mathrm{~Hz}$, 1H), $2.66-2.52(\mathrm{~m}, 2 \mathrm{H}), 2.04(\mathrm{~s}, 3 \mathrm{H}), 1.96-1.89(\mathrm{~m}, 1 \mathrm{H}), 1.84-1.74(\mathrm{~m}, 1 \mathrm{H}) .{ }_{-}^{13} \mathrm{C}$ NMR $(126 \mathrm{MHz}$, $\left.\mathrm{CDCl}_{3}\right) \delta 175.4,145.5,132.2,128.7,118.9,110.9,59.5,52.0,51.6,32.8,30.6,15.4$. FT-IR $\left(\mathrm{cm}^{-1}\right.$, neat, ATR) 3329, 2951, 2917, 2845, 2227, 1732, 1609, 1505, 1435, 1337, 1201, 1169, 1019, 989, 821, 752, 571, 548. HRMS (ESI-TOF) calcd for $\left(\mathrm{C}_{14} \mathrm{H}_{19} \mathrm{~N}_{2} \mathrm{O}_{2} \mathrm{~S}\right)[\mathrm{M}+\mathrm{H}]^{+} 279.1167$, found 279.1174.

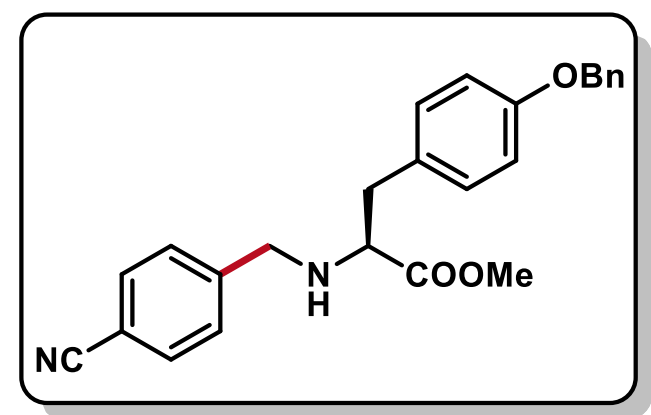

Methyl (S)-3-(4-(Benzyloxy)phenyl)-2-((4-cyanobenzyl) amino)propanoate $(2 \mathrm{~s}, 117.9 \mathrm{mg}, 98 \%)$ was prepared following General Procedure C. This product was obtained as a pale-yellow oil. ${ }^{1} \mathrm{H}$ NMR $\left(400 \mathrm{MHz}, \mathrm{CDCl}_{3}\right) \delta 7.52(\mathrm{~d}, \mathrm{~J}=8.1$ $\mathrm{Hz}, 2 \mathrm{H}), 7.44(\mathrm{~d}, J=7.1 \mathrm{~Hz}, 2 \mathrm{H}), 7.39(\mathrm{t}, J=7.3 \mathrm{~Hz}, 2 \mathrm{H}), 7.36$ - $7.28(\mathrm{~m}, 3 \mathrm{H}), 7.08(\mathrm{~d}, J=8.6 \mathrm{~Hz}, 2 \mathrm{H}), 6.91(\mathrm{~d}, J=8.6 \mathrm{~Hz}, 2 \mathrm{H}), 5.06(\mathrm{~s}, 2 \mathrm{H}), 3.91(\mathrm{~d}, J=14.1 \mathrm{~Hz}, 1 \mathrm{H})$, $3.68(\mathrm{~s}, 3 \mathrm{H}), 3.66(\mathrm{~d}, J=14.7 \mathrm{~Hz}, 1 \mathrm{H}), 3.42(\mathrm{dd}, J=7.5,5.9 \mathrm{~Hz}, 1 \mathrm{H}), 2.95(\mathrm{dd}, J=13.6,5.9 \mathrm{~Hz}, 1 \mathrm{H})$, $2.87(\mathrm{dd}, J=13.7,7.5 \mathrm{~Hz}, 1 \mathrm{H}), 1.88(\mathrm{br}, 1 \mathrm{H}) .{ }^{13} \mathrm{C}$ NMR $\left(101 \mathrm{MHz}, \mathrm{CDCl}_{3}\right) \delta$ 174.9, 157.7, 145.5, 137.1, 132.1, 130.3, 129.4, 128.6, 128.6, 128.0, 127.5, 119.0, 114.9, 110.8, 70.0, 62.2, 51.8, 51.4, 38.9. FT-IR $\left(\mathrm{cm}^{-1}\right.$, neat, ATR) 3338, 3033, 2950, 2227, 1733, 1610, 1584, 1511, 1455, 1381, 1297, 1240, 1175, 1133, 1081, 1019, 917, 820, 738, 697, 549. HRMS (ESI-TOF) calcd for $\left(\mathrm{C}_{25} \mathrm{H}_{25} \mathrm{~N}_{2} \mathrm{O}_{3}\right)[\mathrm{M}+\mathrm{H}]^{+} 401.1865$, found 401.1866. 


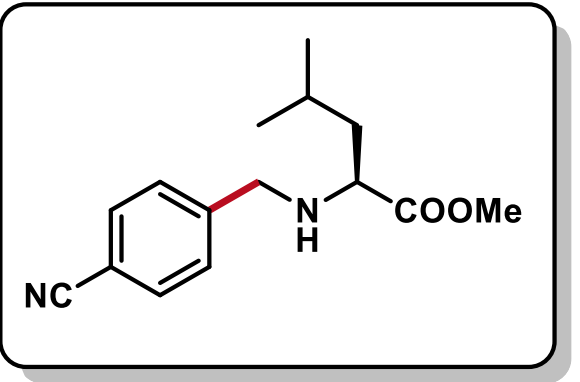

Methyl (4-Cyanobenzyl)-L-leucinate (2t, $65.4 \mathrm{mg}, 84 \%)$ was prepared following General Procedure C. This product was obtained as a pale-yellow oil. ${ }^{1} \mathrm{H}$ NMR $\left(400 \mathrm{MHz}, \mathrm{CDCl}_{3}\right) \delta 7.58(\mathrm{~d}$, $J=8.2 \mathrm{~Hz}, 2 \mathrm{H}), 7.44(\mathrm{~d}, J=8.0 \mathrm{~Hz}, 2 \mathrm{H}), 3.88(\mathrm{~d}, J=14.0 \mathrm{~Hz}, 1 \mathrm{H})$, $3.71(\mathrm{~s}, 3 \mathrm{H}), 3.62(\mathrm{~d}, J=14.1 \mathrm{~Hz}, 1 \mathrm{H}), 3.23(\mathrm{t}, J=7.2 \mathrm{~Hz}, 1 \mathrm{H}), 2.00$ $-1.67(\mathrm{~m}, 1 \mathrm{H}), 1.78(\mathrm{br}, 1 \mathrm{H}), 1.46(\mathrm{t}, J=7.2 \mathrm{~Hz}, 2 \mathrm{H}), 0.90(\mathrm{~d}, J=6.7 \mathrm{~Hz}, 3 \mathrm{H}), 0.84(\mathrm{~d}, J=6.6 \mathrm{~Hz}, 3 \mathrm{H})$. ${ }^{13} \mathrm{C}$ NMR $\left(101 \mathrm{MHz}, \mathrm{CDCl}_{3}\right) \delta$ 176.3, 145.8, 132.2, 128.8, 119.1, 110.9, 59.4, 51.8, 51.7, 42.9, 24.9, 22.9, 22.1. FT-IR ( $\mathrm{cm}^{-1}$, neat, ATR) 3341, 2955, 2870, 2228, 1732, 1609, 1463, 1435, 1368, 1334, 1309, 1270 , 1197, 1172, 1152, 1019, 992, 822, 775, 751, 547. HRMS (ESI-TOF) calcd for $\left(\mathrm{C}_{15} \mathrm{H}_{21} \mathrm{~N}_{2} \mathrm{O}_{2}\right)[\mathrm{M}+\mathrm{H}]^{+}$ 261.1603, found 261.1609.

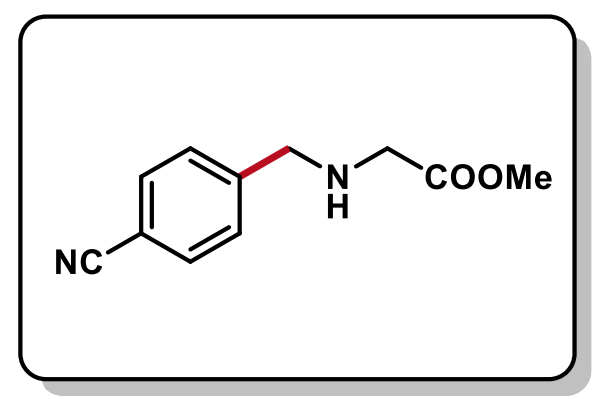

Methyl (4-Cyanobenzyl)glycinate (2u, $54.3 \mathrm{mg}, 89 \%)$ was prepared following General Procedure C. This product was obtained as a pale-yellow oil. ${ }^{1} \mathrm{H}$ NMR $\left(400 \mathrm{MHz}, \mathrm{CDCl}_{3}\right) \delta 7.60$ (d, $J=8.1 \mathrm{~Hz}, 2 \mathrm{H}), 7.45(\mathrm{~d}, J=8.0 \mathrm{~Hz}, 2 \mathrm{H}), 3.86(\mathrm{~s}, 2 \mathrm{H}), 3.72(\mathrm{~s}, 3 \mathrm{H})$, 3.40 (s, 2H), 1.97 (br, 1H). ${ }^{13} \mathrm{C}$ NMR $\left(101 \mathrm{MHz}, \mathrm{CDCl}_{3}\right) \delta 172.8$, 145.3, 132.4, 128.8, 119.0, 111.1, 52.8, 52.0, 50.0. FT-IR ( $\mathrm{cm}^{-1}$, neat, ATR) 3339, 2953, 2228, 1740, 1609, 1505, 1437, 1415, 1368, 1206, 1144, 993, 820, 550. HRMS (ESI-TOF) calcd for $\left(\mathrm{C}_{11} \mathrm{H}_{13} \mathrm{~N}_{2} \mathrm{O}_{2}\right)$ $[\mathrm{M}+\mathrm{H}]^{+}$205.0977, found 205.0987.

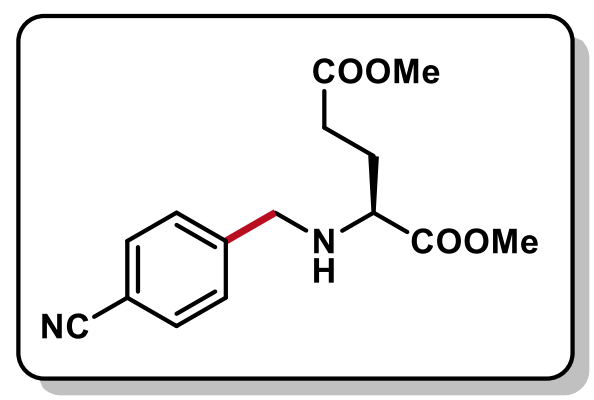

Dimethyl (4-Cyanobenzyl)-L-glutamate (2v, $80.9 \mathrm{mg}, 93 \%)$ was prepared following General Procedure C. This product was obtained as a pale-yellow oil. ${ }^{1} \mathrm{H}$ NMR $\left(500 \mathrm{MHz}, \mathrm{CDCl}_{3}\right) \delta 7.56(\mathrm{~d}$, $J=8.0 \mathrm{~Hz}, 2 \mathrm{H}), 7.41(\mathrm{~d}, J=7.9 \mathrm{~Hz}, 2 \mathrm{H}), 3.87(\mathrm{~d}, J=14.1 \mathrm{~Hz}, 1 \mathrm{H})$, $3.71(\mathrm{~s}, 3 \mathrm{H}), 3.62(\mathrm{~s}, 3 \mathrm{H}), 3.61(\mathrm{~d}, J=14.0 \mathrm{~Hz}, 1 \mathrm{H}), 3.21(\mathrm{dd}, J=$ 8.7, $5.2 \mathrm{~Hz}, 1 \mathrm{H}), 2.44(\mathrm{t}, J=7.3 \mathrm{~Hz}, 2 \mathrm{H}), 2.05-1.95(\mathrm{~m}, 1 \mathrm{H}), 1.88(\mathrm{br}, 1 \mathrm{H}), 1.90-1.81(\mathrm{~m}, 1 \mathrm{H}) .{ }^{13} \mathrm{C}$ 
NMR $\left(126 \mathrm{MHz}, \mathrm{CDCl}_{3}\right) \delta$ 175.1, 173.5, 145.5, 132.2, 128.7, 118.9, 110.9, 59.9, 52.0, 51.7, 51.6, 30.5, 28.3. FT-IR ( $\mathrm{cm}^{-1}$, neat, ATR) 2952, 2228, 1736, 1683, 1609, 1506, 1437, 1407, 1355, 1332, 1201, 1171 , 1117, 1042, 992, 925, 854, 820, 753, 667, 549. HRMS (ESI-TOF) calcd for $\left(\mathrm{C}_{15} \mathrm{H}_{19} \mathrm{~N}_{2} \mathrm{O}_{4}\right)[\mathrm{M}+\mathrm{H}]^{+}$ 291.1345, found 291.1362.

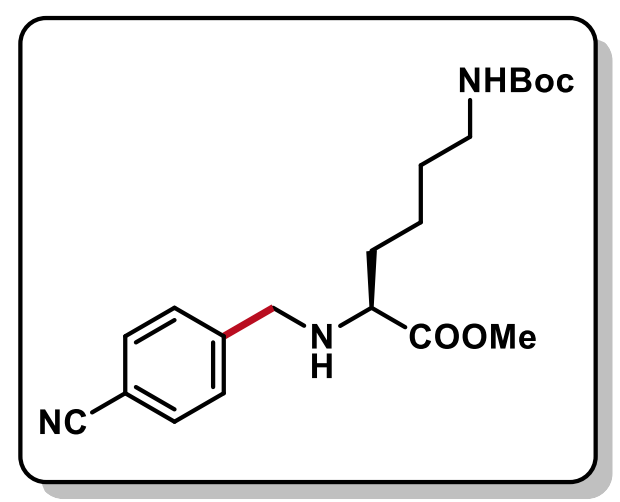

Methyl $\quad N^{6}$-(tert-Butoxycarbonyl)- $N^{2}$-(4-cyanobenzyl)-Llysinate $(2 \mathbf{w}, 88.0 \mathrm{mg}, 78 \%)$ was prepared following General Procedure C. This product was obtained as a pale-yellow oil. ${ }^{1} \mathbf{H}$ NMR $\left(400 \mathrm{MHz}, \mathrm{CDCl}_{3}\right) \delta 7.54(\mathrm{~d}, J=7.9 \mathrm{~Hz}, 2 \mathrm{H}), 7.39(\mathrm{~d}, J=$ $7.9 \mathrm{~Hz}, 2 \mathrm{H}), 4.47(\mathrm{br}, 1 \mathrm{H}), 3.83(\mathrm{~d}, J=14.0 \mathrm{~Hz}, 1 \mathrm{H}), 3.66(\mathrm{~s}, 3 \mathrm{H})$, $3.59(\mathrm{~d}, J=14.2 \mathrm{~Hz}, 1 \mathrm{H}), 3.14(\mathrm{t}, J=6.6 \mathrm{~Hz}, 1 \mathrm{H}), 3.03(\mathrm{q}, J=6.4$ $\mathrm{Hz}, 2 \mathrm{H}), 2.19(\mathrm{br}, 1 \mathrm{H}), 1.69-1.51(\mathrm{~m}, 2 \mathrm{H}), 1.45-1.31(\mathrm{~m}, 4 \mathrm{H}), 1.36(\mathrm{~s}, 9 \mathrm{H}) .{ }^{13} \mathrm{C} \mathrm{NMR}\left(101 \mathrm{MHz}, \mathrm{CDCl}_{3}\right)$ $\delta 175.6,156.1,145.5,132.3,128.9,119.0,111.0,79.3,60.7,52.0,51.7,40.4,33.2,29.9,28.5,23.1$. FT-IR (cm ${ }^{-1}$, neat, ATR) 3358, 2933, 2864, 2228, 1732, 1705, 1513, 1455, 1413, 1391, 1365, 1248, 1167, 1041, 992, 850, 821, 779, 548. HRMS (ESI-TOF) calcd for $\left(\mathrm{C}_{20} \mathrm{H}_{30} \mathrm{~N}_{3} \mathrm{O}_{4}\right)[\mathrm{M}+\mathrm{H}]^{+} 376.2236$, found 376.2252 .

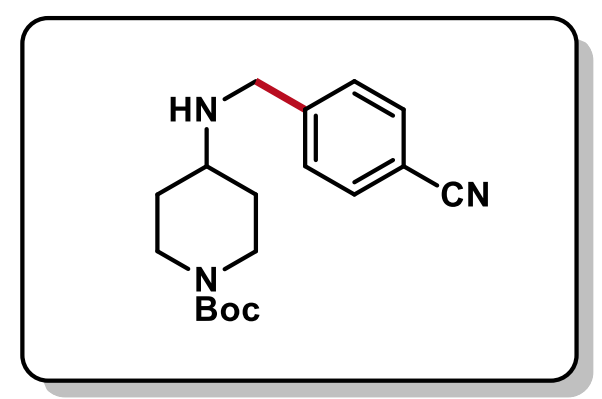

tert-Butyl 4-((4-Cyanobenzyl)amino)piperidine-1-carboxylate (2x, $73.9 \mathrm{mg}, 78 \%$ ) was prepared following General Procedure C. This product was obtained as a pale-yellow oil. ${ }^{1} \mathbf{H}$ NMR $(600 \mathrm{MHz}$, $\left.\mathrm{CDCl}_{3}\right) \delta 7.58(\mathrm{~d}, J=8.2 \mathrm{~Hz}, 2 \mathrm{H}), 7.44(\mathrm{~d}, J=8.0 \mathrm{~Hz}, 2 \mathrm{H}), 4.00-$ $3.96(\mathrm{~m}, 2 \mathrm{H}), 3.86(\mathrm{~s}, 2 \mathrm{H}), 2.87-2.73(\mathrm{~m}, 2 \mathrm{H}), 2.66-2.58(\mathrm{~m}, 1 \mathrm{H})$, $1.83(\mathrm{~d}, J=11.0 \mathrm{~Hz}, 2 \mathrm{H}), 1.43(\mathrm{~s}, 9 \mathrm{H}), 1.32-1.16(\mathrm{~m}, 2 \mathrm{H}), 1.25(\mathrm{br}, 1 \mathrm{H}) .{ }^{13} \mathrm{C} \mathbf{N M R}\left(151 \mathrm{MHz}, \mathrm{CDCl}_{3}\right) \delta$ 154.9, 146.5, 132.3, 128.6, 119.0, 110.8, 79.5, 54.5, 50.4, 42.6, 41.8, 28.5. FT-IR (cm ${ }^{-1}$, neat, ATR) $3320,2975,2932,2853,2227,1683,1608,1468,1449,1423,1365,1275,1236,1169,1139,1007,937$, 867, 821, 770, 549. HRMS (ESI-TOF) calcd for $\left(\mathrm{C}_{18} \mathrm{H}_{26} \mathrm{~N}_{3} \mathrm{O}_{2}\right)[\mathrm{M}+\mathrm{H}]^{+} 316.2025$, found 316.2032. 


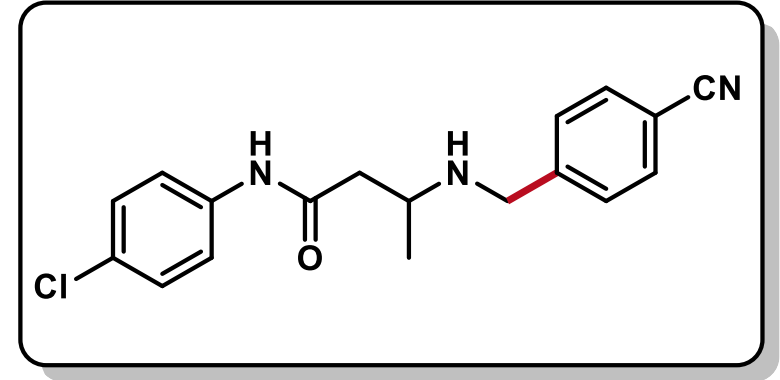

\section{N-(4-Chlorophenyl)-3-((4-cyanobenzyl)amino)buta-}

namide (2y, $97.3 \mathrm{mg}, 99 \%)$ was prepared following

General Procedure C. This product was obtained as a pale-yellow oil. ${ }^{1} \mathrm{H}$ NMR $\left(500 \mathrm{MHz}, \mathrm{CDCl}_{3}\right) \delta 10.04$ (s, 1H), $7.63(\mathrm{~d}, J=7.8 \mathrm{~Hz}, 2 \mathrm{H}), 7.44(\mathrm{~d}, J=7.8 \mathrm{~Hz}, 2 \mathrm{H})$,

$7.39(\mathrm{~d}, J=8.3 \mathrm{~Hz}, 2 \mathrm{H}), 7.25(\mathrm{~d}, J=8.4 \mathrm{~Hz}, 2 \mathrm{H}), 3.98(\mathrm{~d}, J=13.6 \mathrm{~Hz}, 1 \mathrm{H}), 3.88(\mathrm{~d}, J=13.7 \mathrm{~Hz}, 1 \mathrm{H})$, $3.22-3.18(\mathrm{~m}, 1 \mathrm{H}), 2.57(\mathrm{dd}, J=16.1,3.5 \mathrm{~Hz}, 1 \mathrm{H}), 2.37(\mathrm{dd}, J=16.0,7.5 \mathrm{~Hz}, 1 \mathrm{H}), 1.63(\mathrm{br}, 1 \mathrm{H}), 1.26$ $(\mathrm{d}, J=6.5 \mathrm{~Hz}, 3 \mathrm{H}) .{ }^{13} \mathrm{C}$ NMR $\left(126 \mathrm{MHz}, \mathrm{CDCl}_{3}\right) \delta 169.9,145.0,136.9,132.6,129.1,128.9,128.8,121.0$, 118.7, 111.4, 50.6, 50.5, 42.6, 20.5. FT-IR ( $\mathrm{cm}^{-1}$, neat, ATR): 3358, 2933, 2864, 2228, 1732, 1705, 1680, $1513,1455,1365,1236,1167,937,850,821,779,548$. HRMS (ESI-TOF) calcd for $\left(\mathrm{C}_{18} \mathrm{H}_{19} \mathrm{CIN}{ }_{3} \mathrm{O}\right)$ $[\mathrm{M}+\mathrm{H}]^{+} 328.1217$, found 328.1209.

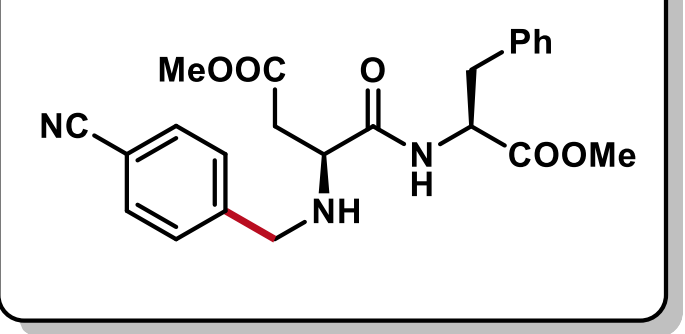

Methyl (S)-3-((4-Cyanobenzyl)amino)-4-(((S)-1-methoxy1-oxo-3-phenylpropan-2-yl)amino)-4-oxobutanoate (2z, $80.3 \mathrm{mg}, 63 \%$ ) was prepared following General Procedure C. This product was obtained as a pale-yellow oil. ${ }^{1} \mathbf{H}$ NMR $\left(400 \mathrm{MHz}, \mathrm{CDCl}_{3}\right) \delta 7.63(\mathrm{~d}, J=8.6 \mathrm{~Hz}, 1 \mathrm{H}), 7.56(\mathrm{~d}, J=7.9$ $\mathrm{Hz}, 2 \mathrm{H}), 7.34-7.27(\mathrm{~m}, 3 \mathrm{H}), 7.30-7.22(\mathrm{~m}, 2 \mathrm{H}), 7.15-7.09(\mathrm{~m}, 2 \mathrm{H}), 4.89(\mathrm{dt}, J=8.5,6.0 \mathrm{~Hz}, 1 \mathrm{H})$, $3.80-3.60(\mathrm{~m}, 2 \mathrm{H}), 3.73(\mathrm{~s}, 3 \mathrm{H}), 3.67(\mathrm{~s}, 3 \mathrm{H}), 3.43(\mathrm{dd}, J=8.2,4.0 \mathrm{~Hz}, 1 \mathrm{H}), 3.21-3.07(\mathrm{~m}, 2 \mathrm{H}), 2.75$ $(\mathrm{dd}, J=16.8,4.0 \mathrm{~Hz}, 1 \mathrm{H}), 2.58(\mathrm{dd}, J=16.8,8.1 \mathrm{~Hz}, 1 \mathrm{H}), 2.19(\mathrm{br}, 1 \mathrm{H}) .{ }^{13} \mathrm{C}$ NMR $\left(101 \mathrm{MHz}, \mathrm{CDCl}_{3}\right) \delta$ $172.1,172.0,171.9,144.7,135.9,132.4,129.4,128.9,128.7,127.3,118.9,111.2,58.6,52.6,52.5,52.1$, 51.6, 38.0, 35.9. FT-IR (cm-1 ${ }^{-1}$ neat, ATR) 3333, 3030, 2953, 2228, 1737, 1672, 1608, 1508, 1437, 1361, $1204,1175,1128,1080,1019,816,747,702,548,494$. HRMS (ESI-TOF) calcd for $\left(\mathrm{C}_{23} \mathrm{H}_{26} \mathrm{~N}_{3} \mathrm{O}_{5}\right)[\mathrm{M}+\mathrm{H}]^{+}$ 424.1872, found 424.1884 . 


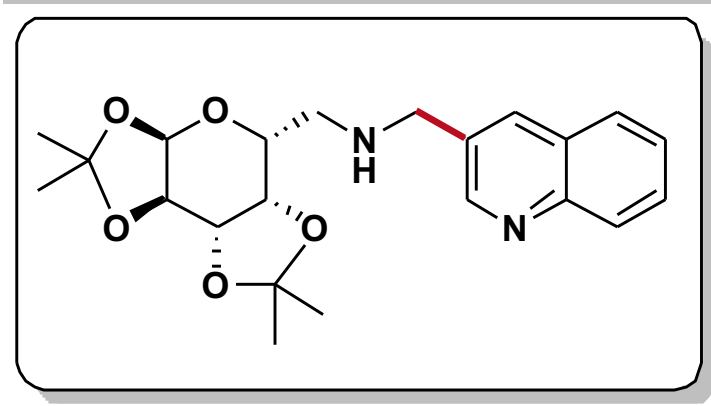

1-(Quinolin-3-yl)-N-(((3aR,5R,5aS,8aS,8bR)-2,2,7,7-tetra methyltetrahydro-5H-bis([1,3]dioxolo)[4,5-b:4',5'-d]pyra n-5-yl)methyl)methanamine (2aa, $93.7 \mathrm{mg}, 78 \%$ ) was prepared following General Procedure C. This product was obtained as a pale-yellow oil. ${ }^{1} \mathrm{H}$ NMR $\left(500 \mathrm{MHz}^{\mathrm{CDCl}} 3\right) \delta$ $8.90(\mathrm{~s}, 1 \mathrm{H}), 8.08(\mathrm{~d}, J=8.3 \mathrm{~Hz}, 2 \mathrm{H}), 7.78(\mathrm{~d}, J=8.1 \mathrm{~Hz}, 1 \mathrm{H}), 7.67(\mathrm{t}, J=7.8 \mathrm{~Hz}, 1 \mathrm{H}), 7.52(\mathrm{t}, J=7.6$ $\mathrm{Hz}, 1 \mathrm{H}), 5.54(\mathrm{~d}, J=5.0 \mathrm{~Hz}, 1 \mathrm{H}), 4.59(\mathrm{~d}, J=8.0 \mathrm{~Hz}, 1 \mathrm{H}), 4.31(\mathrm{~d}, J=5.2 \mathrm{~Hz}, 1 \mathrm{H}), 4.18(\mathrm{~d}, J=8.1 \mathrm{~Hz}$, 1H), $4.03-4.00(\mathrm{~m}, 2 \mathrm{H}), 3.98-3.85(\mathrm{~m}, 1 \mathrm{H}), 2.98(\mathrm{dd}, J=12.5,8.7 \mathrm{~Hz}, 1 \mathrm{H}), 2.81(\mathrm{dd}, J=12.7,4.0 \mathrm{~Hz}$, 1H), $2.07(\mathrm{br}, 1 \mathrm{H}), 1.54(\mathrm{~s}, 3 \mathrm{H}), 1.43(\mathrm{~s}, 3 \mathrm{H}), 1.33(\mathrm{~s}, 3 \mathrm{H}), 1.32(\mathrm{~s}, 3 \mathrm{H}) .{ }^{13} \mathrm{C}$ NMR $\left(126 \mathrm{MHz}, \mathrm{CDCl}_{3}\right) \delta$ $151.8,147.7,134.6,133.1,129.4,129.1$, 128.2, 127.7, 126.7, 109.4, 108.7, 96.6, 72.1, 71.0, 70.8, 67.1, 51.3, 49.2, 26.3, 26.1, 25.1, 24.5. FT-IR (cm², neat, ATR): 3325, 2975, 1732, 1623, 1457, 1247, 1221 , 1151, 903. HRMS (ESI-TOF) calcd for $\left(\mathrm{C}_{22} \mathrm{H}_{29} \mathrm{~N}_{2} \mathrm{O}_{5}\right)[\mathrm{M}+\mathrm{H}]^{+} 401.2076$, found 401.2067.

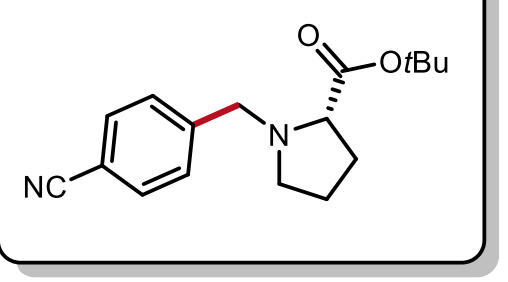

tert-Butyl (4-Cyanobenzyl)-L-prolinate (3a, $72.8 \mathrm{mg}, 85 \%)$ was prepared following General Procedure D. This product was obtained as a pale-yellow oil. ${ }^{1} \mathrm{H}$ NMR $\left(500 \mathrm{MHz}, \mathrm{CDCl}_{3}\right) \delta 7.56(\mathrm{~d}, \mathrm{~J}=8.0 \mathrm{~Hz}$, 2H), $7.46(\mathrm{~d}, J=7.9 \mathrm{~Hz}, 2 \mathrm{H}), 4.01(\mathrm{~d}, J=13.6 \mathrm{~Hz}, 1 \mathrm{H}), 3.52(\mathrm{~d}, J=13.6$ $\mathrm{Hz}, 1 \mathrm{H}), 3.16(\mathrm{dd}, J=8.8,5.9 \mathrm{~Hz}, 1 \mathrm{H}), 2.93(\mathrm{td}, J=8.3,3.4 \mathrm{~Hz}, 1 \mathrm{H})$, $2.33(\mathrm{q}, J=8.1 \mathrm{~Hz}, 1 \mathrm{H}), 2.14-2.03(\mathrm{~m}, 1 \mathrm{H}), 1.95-1.86(\mathrm{~m}, 1 \mathrm{H}), 1.85-1.79(\mathrm{~m}, 1 \mathrm{H}), 1.78-1.70(\mathrm{~m}$, 1H), $1.41(\mathrm{~s}, 9 \mathrm{H}) .{ }^{13} \mathrm{C}$ NMR (126 MHz, $\left.\mathrm{CDCl}_{3}\right) \delta 173.2,145.1,132.2,129.5,119.1,110.8,80.9,66.0$, 58.2, 53.3, 29.3, 28.2, 23.2. FT-IR (cm ${ }^{-1}$, neat, ATR): 3406, 2978, 2229, 1713, 1609, 1550, 1503, 1457 , 1414, 1393, 1367, 1251, 1221, 1150, 1020, 842, 822, 772, 548, 530. HRMS (ESI-TOF) calcd for $\left(\mathrm{C}_{17} \mathrm{H}_{23} \mathrm{~N}_{2} \mathrm{O}_{2}\right)[\mathrm{M}+\mathrm{H}]^{+}$287.1760, found 287.1758. 


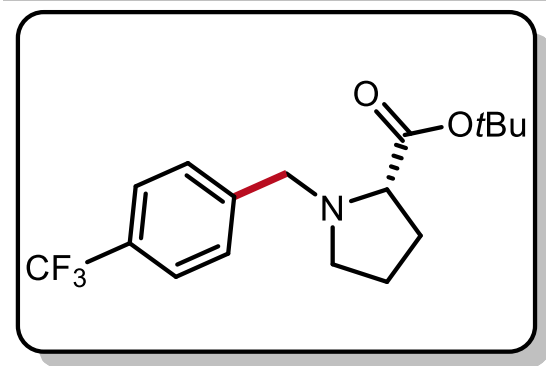

tert-Butyl (4-(Trifluoromethyl)benzyl)-L-prolinate (3b, $94.8 \mathrm{mg}$, 96\%) was prepared following General Procedure D. This product was obtained as a pale-yellow oil. ${ }^{1} \mathrm{H}$ NMR $\left(400 \mathrm{MHz}, \mathrm{CDCl}_{3}\right) \delta 7.54$ (d, $J=8.1 \mathrm{~Hz}, 2 \mathrm{H}), 7.47(\mathrm{~d}, J=8.1 \mathrm{~Hz}, 2 \mathrm{H}), 4.02(\mathrm{~d}, J=13.3 \mathrm{~Hz}, 1 \mathrm{H})$, $3.56(\mathrm{~d}, J=13.3 \mathrm{~Hz}, 1 \mathrm{H}), 3.16(\mathrm{dd}, J=8.8,5.9 \mathrm{~Hz}, 1 \mathrm{H}), 2.97$ (ddd, $J$ $=8.8,7.6,3.4 \mathrm{~Hz}, 1 \mathrm{H}), 2.36(\mathrm{dt}, J=8.9,7.8 \mathrm{~Hz}, 1 \mathrm{H}), 2.18-2.04(\mathrm{~m}, 1 \mathrm{H}), 1.98-1.81(\mathrm{~m}, 2 \mathrm{H}), 1.80-$ $1.71(\mathrm{~m}, 1 \mathrm{H}), 1.43(\mathrm{~s}, 9 \mathrm{H}) .{ }^{13} \mathrm{C}$ NMR $\left(101 \mathrm{MHz}, \mathrm{CDCl}_{3}\right) \delta 173.4,143.5,129.9,129.5,129.3,129.2,128.9$, $128.5,125.8,125.3,125.3,125.3,125.2,123.1,120.4,80.8,66.1,58.2,53.3,29.4,28.3,23.2 .{ }^{19} \mathrm{~F}$ NMR (376 MHz, $\left.\mathrm{CDCl}_{3}\right) \delta$-62.4. FT-IR (cm ${ }^{-1}$, neat, ATR): 2976, 1737, 1619, 1457, 1489, 1392, 1367, 1323 , 1249, 1216, 1152, 1122. HRMS (ESI-TOF) calcd for $\left(\mathrm{C}_{17} \mathrm{H}_{23} \mathrm{~F}_{3} \mathrm{NO}_{2}\right)[\mathrm{M}+\mathrm{H}]^{+} 330.1681$, found 330.1683.

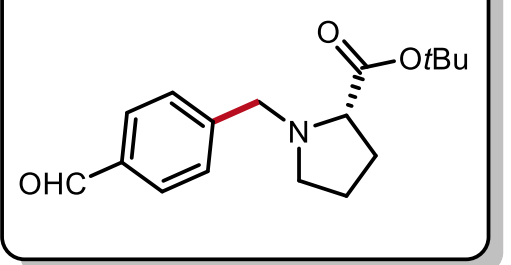

tert-Butyl (4-Formylbenzyl)-L-prolinate (3c, $80.0 \mathrm{mg}, 92 \%)$ was prepared following General Procedure D. This product was obtained as a pale-yellow oil. ${ }^{1} \mathrm{H}$ NMR $\left(600 \mathrm{MHz}, \mathrm{CDCl}_{3}\right) \delta 9.99(\mathrm{~s}, 1 \mathrm{H}), 7.82(\mathrm{~d}$, $J=8.1 \mathrm{~Hz}, 2 \mathrm{H}), 7.54(\mathrm{~d}, J=8.0 \mathrm{~Hz}, 2 \mathrm{H}), 4.07(\mathrm{~d}, J=13.5 \mathrm{~Hz}, 1 \mathrm{H}), 3.57$ $(\mathrm{d}, J=13.5 \mathrm{~Hz}, 1 \mathrm{H}), 3.19(\mathrm{dd}, J=8.8,6.0 \mathrm{~Hz}, 1 \mathrm{H}), 3.02-2.94(\mathrm{~m}, 1 \mathrm{H})$, $2.43-2.34(\mathrm{~m}, 1 \mathrm{H}), 2.19-2.06(\mathrm{~m}, 1 \mathrm{H}), 2.00-1.83(\mathrm{~m}, 2 \mathrm{H}), 1.83-1.74(\mathrm{~m}, 1 \mathrm{H}), 1.45(\mathrm{~s}, 9 \mathrm{H}) .{ }^{13} \mathrm{C}$ NMR $\left(151 \mathrm{MHz}, \mathrm{CDCl}_{3}\right) \delta$ 192.2, 173.3, 146.8, 135.6, 129.9, 129.5, 80.8, 66.1, 58.4, 53.3, 29.3, 28.3, 23.2. FT-IR (cm ${ }^{-1}$, neat, ATR): 2976, 1702, 1607, 1368, 1209, 1154, 847. HRMS (ESI-TOF) calcd for $\left(\mathrm{C}_{17} \mathrm{H}_{24} \mathrm{NO}_{3}\right)[\mathrm{M}+\mathrm{H}]^{+} 290.1756$, found 290.1762.

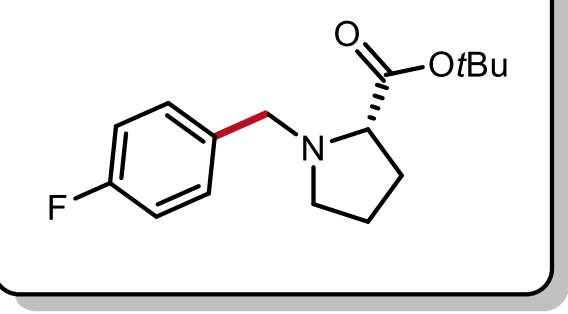

tert-Butyl (4-Fluorobenzyl)-L-prolinate (3d, $77.9 \mathrm{mg}, 93 \%$ ) was prepared following General Procedure D. This product was obtained as a pale-yellow oil. ${ }^{1} \mathbf{H}$ NMR $\left(400 \mathrm{MHz}, \mathrm{CDCl}_{3}\right) \delta 7.30$ (dd, $J=8.5,5.7 \mathrm{~Hz}, 2 \mathrm{H}), 6.97(\mathrm{t}, J=8.7 \mathrm{~Hz}, 2 \mathrm{H}), 3.93$ (d, $J=12.8$ $\mathrm{Hz}, 1 \mathrm{H}), 3.46$ (d, $J=12.8 \mathrm{~Hz}, 1 \mathrm{H}), 3.12(\mathrm{dd}, J=8.9,5.9 \mathrm{~Hz}, 1 \mathrm{H})$, 
$2.96(\mathrm{ddd}, J=9.0,7.7,3.3 \mathrm{~Hz}, 1 \mathrm{H}), 2.33(\mathrm{td}, J=8.6,6.7 \mathrm{~Hz}, 1 \mathrm{H}), 2.20-2.03(\mathrm{~m}, 1 \mathrm{H}), 1.96-1.81(\mathrm{~m}$, 2H), 1.75 (dtd, $J=7.7,5.0,3.4 \mathrm{~Hz}, 1 \mathrm{H}), 1.44$ (s, 9H). ${ }^{13} \mathrm{C}$ NMR $\left(101 \mathrm{MHz}, \mathrm{CDCl}_{3}\right) \delta$ 173.4, 163.3, 160.9, $134.9,134.8,130.7,130.6,115.1,114.9,80.7,65.9,57.8,53.1,29.3,28.2,23.0 .{ }^{19} \mathrm{~F}$ NMR $(376 \mathrm{MHz}$, $\left.\mathrm{CDCl}_{3}\right) \delta$-116.2. FT-IR (cm ${ }^{-1}$, neat, ATR): 2975, 1737, 1724, 1603, 1508, 1479, 1457, 1392, 1367, 1292 1248, 1219. HRMS (ESI-TOF) calcd for $\left(\mathrm{C}_{16} \mathrm{H}_{23} \mathrm{FNO}_{2}\right)[\mathrm{M}+\mathrm{H}]^{+} 280.1713$, found 280.1727.

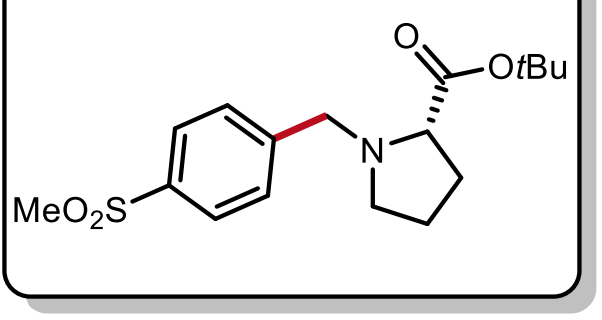

tert-Butyl (4-(Methylsulfonyl)benzyl)-L-prolinate (3e, 91.7 mg, 90\%) was prepared following General Procedure D. This product was obtained as a pale-yellow oil. ${ }^{1} \mathrm{H}$ NMR $\left(400 \mathrm{MHz}, \mathrm{CDCl}_{3}\right) \delta$ $7.86(\mathrm{~d}, J=8.4 \mathrm{~Hz}, 2 \mathrm{H}), 7.56(\mathrm{~d}, J=8.2 \mathrm{~Hz}, 2 \mathrm{H}), 4.05$ (d, $J=13.6$ $\mathrm{Hz}, 1 \mathrm{H}), 3.57$ (d, J = 13.6 Hz, 1H), $3.18(\mathrm{dd}, J=8.8,5.9 \mathrm{~Hz}, 1 \mathrm{H})$, $3.02(\mathrm{~s}, 3 \mathrm{H}), 2.96(\mathrm{td}, J=8.9,8.4,3.4 \mathrm{~Hz}, 1 \mathrm{H}), 2.34(\mathrm{dd}, J=8.6,7.5 \mathrm{~Hz}, 1 \mathrm{H}), 2.15-2.05(\mathrm{~m}, 1 \mathrm{H}), 1.97$ - $1.83(\mathrm{~m}, 2 \mathrm{H}), 1.80-1.73(\mathrm{~m}, 1 \mathrm{H}), 1.43(\mathrm{~s}, 9 \mathrm{H}) .{ }^{13} \mathrm{C}$ NMR $\left(101 \mathrm{MHz}, \mathrm{CDCl}_{3}\right) \delta$ 173.2, 146.1, 139.1, 129.7, 127.4, 80.9, 66.1, 58.0, 53.3, 44.7, 29.3, 28.2, 23.2. FT-IR ( $\mathrm{cm}^{-1}$, neat, ATR): 2975, 2930, 1730, $1457,1409,1392,1367,1304,1252,1215,1145,1089$. HRMS (ESI-TOF) calcd for $\left(\mathrm{C}_{17} \mathrm{H}_{26} \mathrm{NO}_{4} \mathrm{~S}\right)[\mathrm{M}+\mathrm{H}]^{+}$ 340.1583 , found 340.1592 .

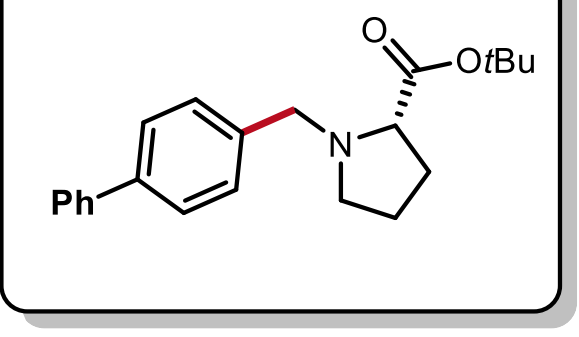

tert-Butyl ([1,1'-Biphenyl]-4-ylmethyl)-L-prolinate (3f, $87.0 \mathrm{mg}$, 86\%) was prepared following General Procedure D. This product was obtained as a pale-yellow oil. ${ }^{1} \mathbf{H}$ NMR $\left(400 \mathrm{MHz}, \mathrm{CDCl}_{3}\right) \delta$ $7.59(\mathrm{dd}, J=8.3,1.3 \mathrm{~Hz}, 2 \mathrm{H}), 7.54(\mathrm{~d}, J=8.2 \mathrm{~Hz}, 2 \mathrm{H}), 7.46-7.39$ $(\mathrm{m}, 4 \mathrm{H}), 7.38-7.29(\mathrm{~m}, 1 \mathrm{H}), 4.02(\mathrm{~d}, J=12.9 \mathrm{~Hz}, 1 \mathrm{H}), 3.57(\mathrm{~d}, J$ $=12.9 \mathrm{~Hz}, 1 \mathrm{H}), 3.18(\mathrm{dd}, J=8.8,5.9 \mathrm{~Hz}, 1 \mathrm{H}), 3.09-3.00(\mathrm{~m}, 1 \mathrm{H}), 2.46-2.34(\mathrm{~m}, 1 \mathrm{H}), 2.21-2.06(\mathrm{~m}$, 1H), $2.01-1.84(\mathrm{~m}, 2 \mathrm{H}), 1.82-1.68(\mathrm{~m}, 1 \mathrm{H}), 1.47(\mathrm{~s}, 9 \mathrm{H}) .{ }^{13} \mathrm{C} \mathrm{NMR}\left(101 \mathrm{MHz}, \mathrm{CDCl}_{3}\right) \delta 173.5,141.2$, 140.0, 138.2, 129.6, 128.8, 127.2, 127.2, 127.1, 80.7, 66.0, 58.2, 53.3, 29.4, 28.3, 23.1. FT-IR $\left(\mathrm{cm}^{-1}\right.$, 
neat, ATR): $2974,2876,2805,1736,1722,1488,1456,1409,1391,1366,1291,1249$. HRMS (ESITOF) calcd for $\left(\mathrm{C}_{22} \mathrm{H}_{28} \mathrm{NO}_{2}\right)[\mathrm{M}+\mathrm{H}]^{+} 338.2120$, found 338.2132 .

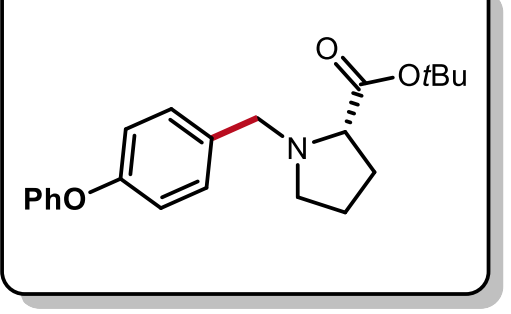

tert-Butyl (4-Phenoxybenzyl)-L-prolinate (3g, $94.0 \mathrm{mg}, 89$ \%) was prepared following General Procedure D. This product was obtained as a pale-yellow oil. ${ }^{1} \mathrm{H}$ NMR $\left(600 \mathrm{MHz}, \mathrm{CDCl}_{3}\right) \delta 7.37-7.26(\mathrm{~m}, 4 \mathrm{H})$, $7.07(\mathrm{tt}, J=7.3,1.1 \mathrm{~Hz}, 1 \mathrm{H}), 6.98(\mathrm{dd}, J=8.7,1.1 \mathrm{~Hz}, 2 \mathrm{H}), 6.96-6.80$ (m, 2H), $3.96(\mathrm{~d}, J=12.8 \mathrm{~Hz}, 1 \mathrm{H}), 3.51(\mathrm{~d}, J=12.8 \mathrm{~Hz}, 1 \mathrm{H}), 3.16(\mathrm{dd}$, $J=8.9,6.0 \mathrm{~Hz}, 1 \mathrm{H}), 3.02(\mathrm{td}, J=8.6,3.2 \mathrm{~Hz}, 1 \mathrm{H}), 2.39(\mathrm{q}, J=8.1 \mathrm{~Hz}, 1 \mathrm{H}), 2.14-2.05(\mathrm{~m}, 1 \mathrm{H}), 1.98-$ $1.90(\mathrm{~m}, 1 \mathrm{H}), 1.90-1.83(\mathrm{~m}, 1 \mathrm{H}), 1.82-1.70(\mathrm{~m}, 1 \mathrm{H}), 1.46(\mathrm{~s}, 9 \mathrm{H}) .{ }^{13} \mathrm{C}$ NMR $\left(151 \mathrm{MHz}, \mathrm{CDCl}_{3}\right) \delta 173.2$, 157.5, 156.2, 130.6, 129.7, 123.1, 118.8, 118.7, 80.7, 65.7, 57.7, 53.1, 29.2, 28.2, 22.9._FT-IR $\left(\mathrm{cm}^{-1}\right.$, neat, ATR): 2976, 1737, 1590, 1506, 1489, 1457, 1392, 1367, 1288, 1234, 1152, 1016, 871, 846, 750 , 693, 504. HRMS (ESI-TOF) calcd for $\left(\mathrm{C}_{22} \mathrm{H}_{28} \mathrm{NO}_{3}\right)[\mathrm{M}+\mathrm{H}]^{+} 354.2069$, found 354.2063 .

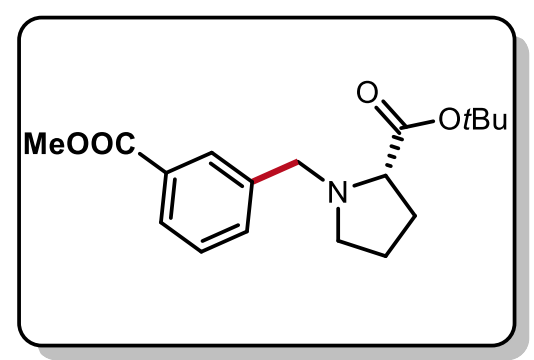

tert-Butyl (3-(Methoxycarbonyl)benzyl)-L-prolinate (3h, $90.4 \mathrm{mg}$, 94\%) was prepared following General Procedure D. This product was obtained as a pale-yellow oil. ${ }^{1} \mathrm{H}$ NMR $\left(500 \mathrm{MHz}, \mathrm{CDCl}_{3}\right) \delta 7.96$ (t, $\mathrm{J}=$ $1.7 \mathrm{~Hz}, 1 \mathrm{H}), 7.89(\mathrm{dt}, J=7.7,1.5 \mathrm{~Hz}, 1 \mathrm{H}), 7.56(\mathrm{dt}, J=7.6,1.5 \mathrm{~Hz}, 1 \mathrm{H})$, $7.35(\mathrm{t}, J=7.7 \mathrm{~Hz}, 1 \mathrm{H}), 4.00(\mathrm{~d}, J=13.0 \mathrm{~Hz}, 1 \mathrm{H}), 3.87(\mathrm{~s}, 3 \mathrm{H}), 3.53(\mathrm{~d}$, $J=13.0 \mathrm{~Hz}, 1 \mathrm{H}), 3.14(\mathrm{dd}, J=8.8,5.9 \mathrm{~Hz}, 1 \mathrm{H}), 2.98-2.90(\mathrm{~m}, 1 \mathrm{H}), 2.35(\mathrm{q}, J=8.0 \mathrm{~Hz}, 1 \mathrm{H}), 2.12-2.01$ $(\mathrm{m}, 1 \mathrm{H}), 1.98-1.88(\mathrm{~m}, 1 \mathrm{H}), 1.91-1.73(\mathrm{~m}, 1 \mathrm{H}), 1.76-1.67(\mathrm{~m}, 1 \mathrm{H}), 1.43(\mathrm{~s}, 9 \mathrm{H}) .{ }^{13} \mathrm{C}$ NMR $(126 \mathrm{MHz}$, $\left.\mathrm{CDCl}_{3}\right)$ $\delta 173.3,167.3,139.6,133.9,130.2,130.1,128.4,80.7,65.9,58.1,53.1,52.1,29.3,28.2,23.1$. FT-IR ( $\mathrm{cm}^{-1}$, neat, ATR): 2977, 1724, 1436, 1369, 1287, 1204, 1154, 1109, 844, 754. HRMS (ESI-TOF) calcd for $\left(\mathrm{C}_{18} \mathrm{H}_{26} \mathrm{NO}_{4}\right)[\mathrm{M}+\mathrm{H}]^{+}$320.1862, found 320.1868. 


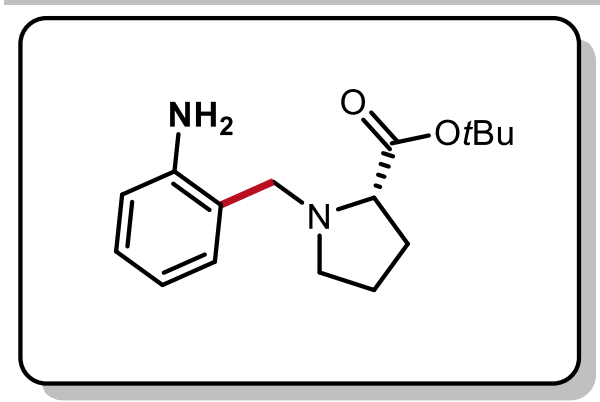

tert-Butyl (2-Aminobenzyl)-L-prolinate (3i, $56.4 \mathrm{mg}, 68 \%)$ was prepared following General Procedure D with the following modifications: 2-iodoaniline was used in place of 2-bromoaniline; two blue Kessil LEDs $(2 \times 30 \mathrm{~W})$ were used in place of $\sim 10 \mathrm{~W}$ blue LEDs, and the reaction time was shortened to $6 \mathrm{~h}$. This product was obtained as a pink solid $\left(\mathrm{mp}=36-37^{\circ} \mathrm{C}\right) .{ }^{1} \mathrm{H}$ NMR $\left(600 \mathrm{MHz}, \mathrm{CDCl}_{3}\right) \delta 7.06(\mathrm{td}, J=7.6,1.6 \mathrm{~Hz}$, 1H), $6.98(\mathrm{~d}, J=6.8 \mathrm{~Hz}, 1 \mathrm{H}), 6.61(\mathrm{t}, J=7.3 \mathrm{~Hz}, 2 \mathrm{H}), 4.86(\mathrm{br}, 2 \mathrm{H}), 4.00(\mathrm{~d}, J=12.2 \mathrm{~Hz}, 1 \mathrm{H}), 3.25(\mathrm{~d}, J$ $=12.2 \mathrm{~Hz}, 1 \mathrm{H}), 3.04(\mathrm{dd}, J=9.1,6.9 \mathrm{~Hz}, 1 \mathrm{H}), 2.90$ (ddd, $J=9.7,7.4,2.8 \mathrm{~Hz}, 1 \mathrm{H}), 2.29-2.19(\mathrm{~m}, 1 \mathrm{H})$, $2.19-2.09(\mathrm{~m}, 1 \mathrm{H}), 1.93-1.84(\mathrm{~m}, 1 \mathrm{H}), 1.84-1.69(\mathrm{~m}, 2 \mathrm{H}), 1.46(\mathrm{~s}, 9 \mathrm{H}) .{ }^{13} \mathrm{C} \mathrm{NMR}\left(151 \mathrm{MHz}, \mathrm{CDCl}_{3}\right)$ $\delta 175.2,148.5,131.2,129.7,124.4$, 118.3, 116.5, 82.2, 67.9, 59.3, 54.5, 31.1, 29.4, 24.4. FT-IR $\left(\mathrm{cm}^{-1}\right.$, neat, ATR): 3443, 3342, 2976, 2801, 1730, 1620, 1495, 1460, 1368, 1290, 1219, 1154, 845, 748, 537. HRMS (ESI-TOF) calcd for $\left(\mathrm{C}_{16} \mathrm{H}_{25} \mathrm{~N}_{2} \mathrm{O}_{2}\right)[\mathrm{M}+\mathrm{H}]^{+} 277.1916$, found 277.1905 .

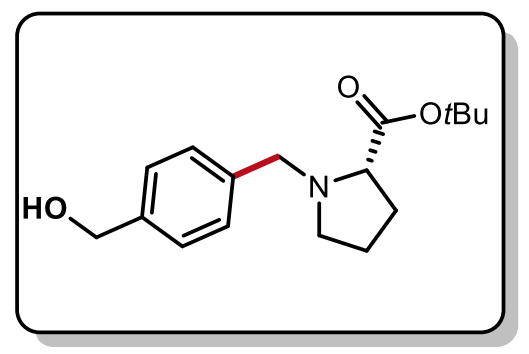

tert-Butyl (4-(Hydroxymethyl)benzyl)-L-prolinate (3j, 52.5 mg, 60\%) was prepared following General Procedure D. This product was obtained as a pale-yellow oil. ${ }^{1} \mathrm{H}$ NMR $\left(500 \mathrm{MHz}, \mathrm{CDCl}_{3}\right) \delta 7.31(\mathrm{~d}, \mathrm{~J}=$ $8.1 \mathrm{~Hz}, 2 \mathrm{H}), 7.28(\mathrm{~d}, J=8.1 \mathrm{~Hz}, 2 \mathrm{H}), 4.64(\mathrm{~s}, 2 \mathrm{H}), 3.96(\mathrm{~d}, J=12.8 \mathrm{~Hz}$, $1 \mathrm{H}), 3.49(\mathrm{~d}, J=12.9 \mathrm{~Hz}, 1 \mathrm{H}), 3.12(\mathrm{dd}, J=8.8,6.1 \mathrm{~Hz}, 1 \mathrm{H}), 2.95(\mathrm{td}$, $J=8.3,3.2 \mathrm{~Hz}, 1 \mathrm{H}), 2.38(\mathrm{br}, 1 \mathrm{H}), 2.34(\mathrm{q}, J=8.2 \mathrm{~Hz}, 1 \mathrm{H}), 2.12-1.99(\mathrm{~m}, 1 \mathrm{H}), 2.01-1.86(\mathrm{~m}, 1 \mathrm{H})$, $1.86-1.77(\mathrm{~m}, 1 \mathrm{H}), 1.79-1.63(\mathrm{~m}, 1 \mathrm{H}), 1.44(\mathrm{~s}, 9 \mathrm{H}) .{ }^{13} \mathrm{C}$ NMR $\left(126 \mathrm{MHz}, \mathrm{CDCl}_{3}\right) \delta 173.4,139.9,138.2$, 129.4, 127.0, 80.7, 65.8, 65.2, 58.1, 53.1, 29.3, 28.3, 23.0. FT-IR ( $\mathrm{cm}^{-1}$, neat, ATR): 3386, 2975, 2874 , 1725, 1513, 1457, 1420, 1392, 1367, 1292, 1250, 1215, 1153, 1044, 1019, 901, 845, 811, 756, 471. HRMS (ESI-TOF) calcd for $\left(\mathrm{C}_{17} \mathrm{H}_{25} \mathrm{NO}_{3} \mathrm{Na}\right)[\mathrm{M}+\mathrm{Na}]^{+} 314.1732$, found 314.1722. 


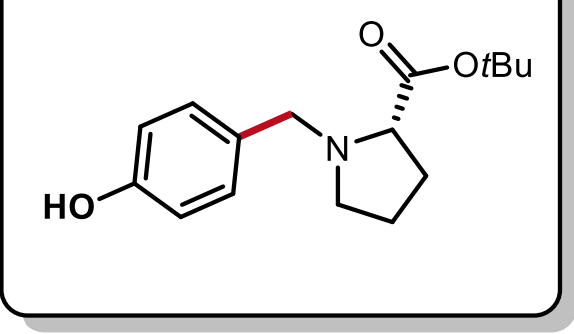

tert-Butyl (4-Hydroxybenzyl)-L-prolinate (3k, $70.6 \mathrm{mg}, 85 \%)$ was prepared following General Procedure D with the following modifications: two blue Kessil LEDs $(2 \times 30 \mathrm{~W})$ were used in place of $\sim 10 \mathrm{~W}$ blue LEDs, and the reaction time was shortened to $6 \mathrm{~h}$.

This product was obtained as a pale-yellow oil. ${ }^{1} \mathrm{H}$ NMR $(500 \mathrm{MHz}$, $\left.\mathrm{CDCl}_{3}\right) \delta 7.14(\mathrm{~d}, J=6.3 \mathrm{~Hz}, 2 \mathrm{H}), 6.72(\mathrm{~d}, J=6.3 \mathrm{~Hz}, 2 \mathrm{H}), 3.85(\mathrm{~d}, J=12.7 \mathrm{~Hz}, 1 \mathrm{H}), 3.48(\mathrm{~d}, J=12.7$ $\mathrm{Hz}, 1 \mathrm{H}), 3.14(\mathrm{t}, J=6.5 \mathrm{~Hz}, 1 \mathrm{H}), 2.99(\mathrm{t}, J=8.1 \mathrm{~Hz}, 1 \mathrm{H}), 2.38(\mathrm{q}, J=7.1,1 \mathrm{H}), 2.13-2.04(\mathrm{~m}, 1 \mathrm{H}), 1.94$ - $1.75(\mathrm{~m}, 2 \mathrm{H}), 1.75-1.70(\mathrm{~m}, 1 \mathrm{H}), 1.45(\mathrm{~s}, 9 \mathrm{H}) .{ }^{13} \mathrm{C}$ NMR $\left(126 \mathrm{MHz}, \mathrm{CDCl}_{3}\right) \delta$ 173.8, 155.5, 130.7, 129.8, 115.4, 81.0, 65.6, 57.7, 53.0, 29.3, 28.2, 22.8. FT-IR ( $\mathrm{cm}^{-1}$, neat, ATR): 3345, 2976, 2878, 2813, 1722, 1614, 1596, 1515, 1455, 1392, 1367, 1223, 1152, 1100, 1031, 837, 780, 747, 637, 509. HRMS (ESI-TOF) calcd for $\left(\mathrm{C}_{16} \mathrm{H}_{24} \mathrm{NO}_{3}\right)[\mathrm{M}+\mathrm{H}]^{+} 278.1756$, found 278.1759 .

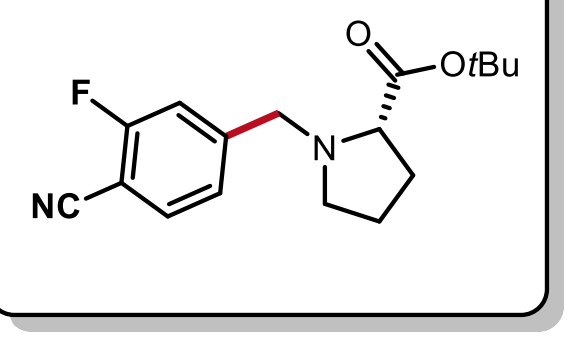

tert-Butyl (4-Cyano-3-fluorobenzyl)-L-prolinate (3I, $88.5 \mathrm{mg}$, 97\%) was prepared following General Procedure D. This product was obtained as a pale-yellow oil. ${ }^{1} \mathrm{H}$ NMR $\left(400 \mathrm{MHz}, \mathrm{CDCl}_{3}\right) \delta 7.54$ (dd, $J=7.9,6.5 \mathrm{~Hz}, 1 \mathrm{H}), 7.29(\mathrm{~d}, J=9.3 \mathrm{~Hz}, 1 \mathrm{H}), 7.26$ (d, $J=7.6$ $\mathrm{Hz}, 1 \mathrm{H}), 4.03(\mathrm{~d}, J=14.2 \mathrm{~Hz}, 1 \mathrm{H}), 3.56(\mathrm{~d}, J=14.1 \mathrm{~Hz}, 1 \mathrm{H}), 3.21$ (dd, $J=8.8,5.7 \mathrm{~Hz}, 1 \mathrm{H}$ ), $2.97(\mathrm{ddd}, J=8.8,7.6,3.6 \mathrm{~Hz}, 1 \mathrm{H}), 2.37(\mathrm{dt}, J=8.8,7.8 \mathrm{~Hz}, 1 \mathrm{H}), 2.22-2.05$ $(\mathrm{m}, 1 \mathrm{H}), 1.99-1.85(\mathrm{~m}, 2 \mathrm{H}), 1.82-1.72(\mathrm{~m}, 1 \mathrm{H}), 1.43(\mathrm{~s}, 9 \mathrm{H}) .{ }^{13} \mathrm{C}$ NMR $\left(101 \mathrm{MHz}, \mathrm{CDCl}_{3}\right) \delta 173.1$, $164.7,162.1,148.9,148.8,133.3,124.9,124.9,116.6,116.4,114.3,99.9,99.7,81.0,66.0,57.8,53.3$, 29.3, 28.2, 23.3. $\left.{ }^{19} \mathrm{~F} \mathrm{NMR} \mathrm{(376} \mathrm{MHz,} \mathrm{CDCl}_{3}\right) \delta$-107.1. FT-IR ( $\mathrm{cm}^{-1}$, neat, ATR): 2976, 2236, 1729, 1622 , $1571,1500,1456,1430,1393,1367,1283,1252$. HRMS (ESI-TOF) calcd for $\left(\mathrm{C}_{17} \mathrm{H}_{22} \mathrm{FN}_{2} \mathrm{O}_{2}\right)[\mathrm{M}+\mathrm{H}]^{+}$ 305.1665 , found 305.1669 . 


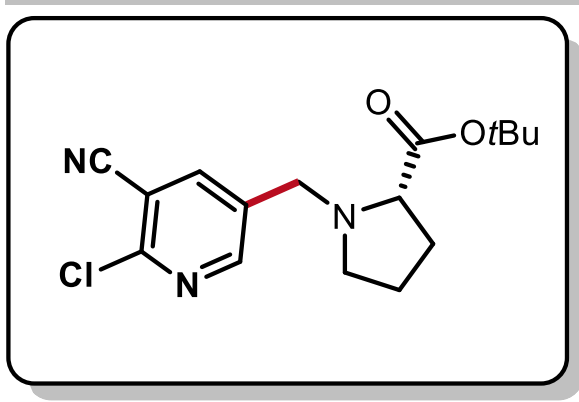

tert-Butyl ((6-Chloro-5-cyanopyridin-3-yl)methyl)-L-prolinate (3m, $43.4 \mathrm{mg}, 45 \%$ ) was prepared following General Procedure D. This product was obtained as a pale-yellow oil. ${ }^{1} \mathbf{H}$ NMR $(400 \mathrm{MHz}$, $\left.\mathrm{CDCl}_{3}\right) \delta 8.51(\mathrm{~d}, J=2.3 \mathrm{~Hz}, 1 \mathrm{H}), 8.12(\mathrm{~d}, J=2.3 \mathrm{~Hz}, 1 \mathrm{H}), 4.00(\mathrm{~d}$, $J=13.9 \mathrm{~Hz}, 1 \mathrm{H}), 3.57(\mathrm{~d}, J=13.9 \mathrm{~Hz}, 1 \mathrm{H}), 3.22(\mathrm{dd}, J=8.8,5.7 \mathrm{~Hz}$, 1H), $2.95(\mathrm{td}, J=8.1,3.6 \mathrm{~Hz}, 1 \mathrm{H}), 2.44-2.34(\mathrm{~m}, 1 \mathrm{H}), 2.21-2.10(\mathrm{~m}, 1 \mathrm{H}), 2.01-1.74(\mathrm{~m}, 3 \mathrm{H}), 1.44$ (s, 9H). ${ }^{13} \mathrm{C}$ NMR $\left(101 \mathrm{MHz}, \mathrm{CDCl}_{3}\right) \delta 172.9,152.7,151.3,143.2,134.9,114.9,110.6,81.3,65.9,54.3$, 53.3, 29.4, 28.2, 23.2. FT-IR (cm ${ }^{-1}$, neat, ATR): 2977, 2236, 1729, 1589, 1558, 1458, 1421, 1403, 1367 , 1287, 1255, 1223. HRMS (ESI-TOF) calcd for $\left(\mathrm{C}_{16} \mathrm{H}_{21} \mathrm{CIN}_{3} \mathrm{O}_{2}\right)[\mathrm{M}+\mathrm{H}]^{+} 322.1322$, found 322.1308.

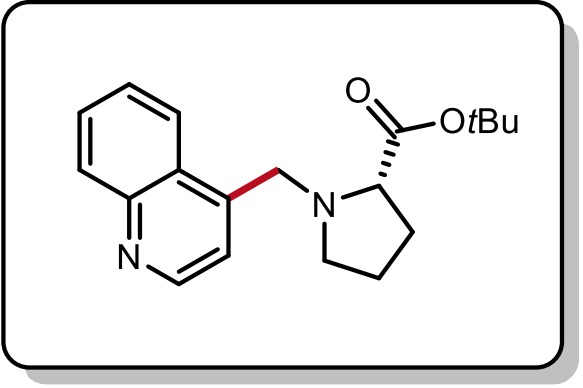

tert-Butyl (Quinolin-4-ylmethyl)-L-prolinate (3n, 80.6 mg, 86\%) was prepared following General Procedure D. This product was obtained as a pale-yellow oil. ${ }^{1} \mathrm{H}$ NMR $\left(400 \mathrm{MHz}, \mathrm{CDCl}_{3}\right) \delta 8.82(\mathrm{~d}$, $J=4.4 \mathrm{~Hz}, 1 \mathrm{H}), 8.43(\mathrm{dd}, J=8.4,1.5 \mathrm{~Hz}, 1 \mathrm{H}), 8.09$ (dd, $J=8.4$, $1.3 \mathrm{~Hz}, 1 \mathrm{H}), 7.68(\mathrm{ddd}, J=8.4,6.8,1.4 \mathrm{~Hz}, 1 \mathrm{H}), 7.55$ (ddd, $J=8.3$, 6.8, $1.3 \mathrm{~Hz}, 1 \mathrm{H}), 7.41(\mathrm{~d}, J=4.3 \mathrm{~Hz}, 1 \mathrm{H}), 4.47(\mathrm{~d}, J=12.8 \mathrm{~Hz}, 1 \mathrm{H}), 3.86(\mathrm{~d}, J=13.5 \mathrm{~Hz}, 1 \mathrm{H}), 3.23$ (dd, $J=8.8,6.1 \mathrm{~Hz}, 1 \mathrm{H}), 2.95-2.81(\mathrm{~m}, 1 \mathrm{H}), 2.39(\mathrm{q}, J=8.1 \mathrm{~Hz}, 1 \mathrm{H}), 2.19-2.05(\mathrm{~m}, 1 \mathrm{H}), 1.99-1.91(\mathrm{~m}$, 1H), $1.89-1.81(\mathrm{~m}, 1 \mathrm{H}), 1.80-1.73(\mathrm{~m}, 1 \mathrm{H}), 1.43(\mathrm{~s}, 9 \mathrm{H}) .{ }^{13} \mathrm{C}$ NMR $\left(101 \mathrm{MHz}, \mathrm{CDCl}_{3}\right) \delta 173.3,150.2$, 148.3, 145.0, 129.8, 129.2, 127.8, 126.5, 124.7, 121.3, 80.9, 66.8, 55.9, 53.5, 29.4, 28.2, 23.2. FT-IR ( $\mathrm{cm}^{-1}$, neat, ATR): 2975, 2807, 1732, 1595, 1507, 1509, 1558, 1425, 1392, 1367, 1291, 1216. HRMS (ESI-TOF) calcd for $\left(\mathrm{C}_{19} \mathrm{H}_{25} \mathrm{~N}_{2} \mathrm{O}_{2}\right)[\mathrm{M}+\mathrm{H}]^{+} 313.1916$, found 313.1907. 


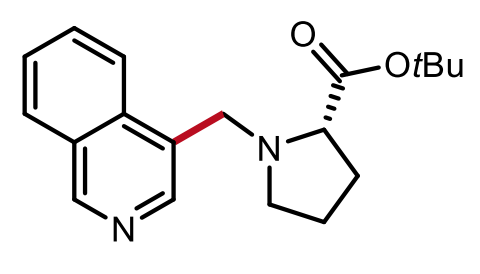

tert-Butyl (Isoquinolin-4-ylmethyl)-L-prolinate (3o, 46.9 mg, $50 \%$ ) was prepared following General Procedure D. This product was obtained as a pale-yellow oil. ${ }^{1} \mathrm{H}$ NMR $\left(400 \mathrm{MHz}, \mathrm{CDCl}_{3}\right) \delta$ $9.17(\mathrm{~s}, 1 \mathrm{H}), 8.59(\mathrm{~d}, J=8.5 \mathrm{~Hz}, 1 \mathrm{H}), 8.41(\mathrm{~s}, 1 \mathrm{H}), 7.96(\mathrm{~d}, J=8.2$ $\mathrm{Hz}, 1 \mathrm{H}), 7.75$ (ddd, $J=8.4,6.9,1.4 \mathrm{~Hz}, 1 \mathrm{H}), 7.61$ (ddd, $J=8.1,6.8$, $1.1 \mathrm{~Hz}, 1 \mathrm{H}), 4.47(\mathrm{~d}, J=12.5 \mathrm{~Hz}, 1 \mathrm{H}), 3.72(\mathrm{~d}, J=12.5 \mathrm{~Hz}, 1 \mathrm{H}), 3.18(\mathrm{dd}, J=8.9,6.5 \mathrm{~Hz}, 1 \mathrm{H}), 2.88-$ $2.78(\mathrm{~m}, 1 \mathrm{H}), 2.37(\mathrm{q}, J=8.4 \mathrm{~Hz}, 1 \mathrm{H}), 2.23-2.06(\mathrm{~m}, 1 \mathrm{H}), 2.01-1.89(\mathrm{~m}, 1 \mathrm{H}), 1.88-1.61(\mathrm{~m}, 2 \mathrm{H})$, 1.47 (s, 9H). ${ }^{13} \mathrm{C}$ NMR $\left(101 \mathrm{MHz}, \mathrm{CDCl}_{3}\right) \delta 173.4,152.9,143.2,135.6,130.5,128.6,128.5,127.8,127.2$, 124.7, 80.8, 66.9, 54.8, 53.4, 29.5, 28.3, 23.0. FT-IR ( $\mathrm{cm}^{-1}$, neat, ATR): 2975, 1732, 1623, 1504, 1457 , 1391, 1367, 1291, 1247, 1221, 1151, 903. HRMS (ESI-TOF) calcd for $\left(\mathrm{C}_{19} \mathrm{H}_{25} \mathrm{~N}_{2} \mathrm{O}_{2}\right)[\mathrm{M}+\mathrm{H}]^{+} 313.1916$, found 313.1919.

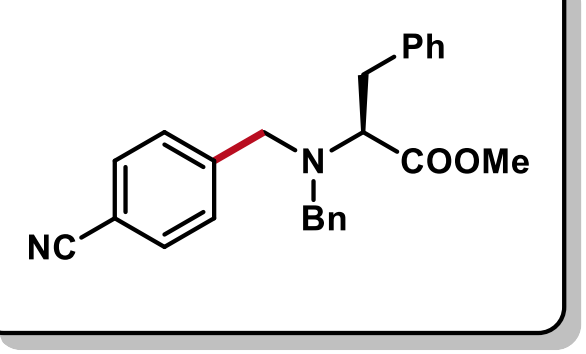

Methyl N-Benzyl-N-(4-cyanobenzyl)-L-phenylalaninate (3q, $100.3 \mathrm{mg}, 87 \%$ ) was prepared following General Procedure D. This product was obtained as a pale-yellow oil. ${ }^{1} \mathbf{H}$ NMR (500 $\left.\mathrm{MHz}_{\mathrm{CDCl}}\right) \delta 7.51-7.46(\mathrm{~m}, 2 \mathrm{H}), 7.30-7.21(\mathrm{~m}, 6 \mathrm{H}), 7.19(\mathrm{~d}$, $J=8.0 \mathrm{~Hz}, 2 \mathrm{H}), 7.17-7.09(\mathrm{~m}, 2 \mathrm{H}), 7.03-6.98(\mathrm{~m}, 2 \mathrm{H}), 3.95(\mathrm{~d}$,

$J=14.8 \mathrm{~Hz}, 1 \mathrm{H}), 3.92(\mathrm{~d}, J=14.0 \mathrm{~Hz}, 1 \mathrm{H}), 3.75(\mathrm{~s}, 3 \mathrm{H}), 3.68-3.64(\mathrm{~m}, 1 \mathrm{H}), 3.62(\mathrm{dd}, J=9.0,6.6 \mathrm{~Hz}$, 1H), $3.56(\mathrm{~d}, J=13.9 \mathrm{~Hz}, 1 \mathrm{H}), 3.12(\mathrm{dd}, J=14.1,6.6 \mathrm{~Hz}, 1 \mathrm{H}), 2.98(\mathrm{dd}, J=14.2,9.0 \mathrm{~Hz}, 1 \mathrm{H}) .{ }^{13} \mathrm{C}$ NMR $\left(126 \mathrm{MHz}, \mathrm{CDCl}_{3}\right) \delta 172.7,145.4,138.7,138.0,132.1,129.5,129.3,128.8,128.5,128.4,127.4,126.7$, 119.1, 110.8, 62.8, 54.9, 54.3, 51.5, 35.9. FT-IR (cm ${ }^{-1}$, neat, ATR) 3028, 2950, 2843, 2227, 1731, 1606, 1495, 1454, 1434, 1359, 1287, 1203, 1166, 1076, 1029, 990, 823, 740, 698, 549. HRMS (ESI-TOF) calcd for $\left(\mathrm{C}_{25} \mathrm{H}_{25} \mathrm{~N}_{2} \mathrm{O}_{2}\right)[\mathrm{M}+\mathrm{H}]^{+} 385.1916$, found 385.1910 . 


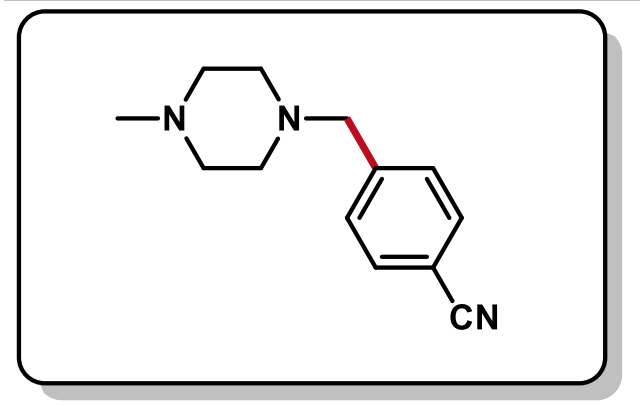

4-((4-Methylpiperazin-1-yl)methyl)benzonitrile (3r, $59.4 \mathrm{mg}$, 92\%) was prepared following General Procedure D with the following modifications: two blue Kessil LEDs $(2 \times 30 \mathrm{~W})$ were used in place of $\sim 10 \mathrm{~W}$ blue LEDs, and the reaction time was shortened to $6 \mathrm{~h}$. The $\mathrm{SiO}_{2}$ column chromatography utilized a gradient elution [flow rate: $30 \mathrm{~mL} / \mathrm{min}, \mathrm{CH}_{2} \mathrm{Cl}_{2}$ (Solvent A), $\mathrm{MeOH}$ (Solvent B)]: 3 min (100\% A), 6 min (100\% A to $80 \%$ A: $20 \%$ B), 3 min (Hold $80 \%$ A: $20 \%$ B), $3 \min (80 \%$ A: $20 \%$ B to $50 \%$ A: $50 \%$ B), 5 min (Hold $50 \%$ A: $50 \%$ B). This product was obtained as a pale-yellow solid ( $\left.m p=69-70{ }^{\circ} \mathrm{C}\right) .{ }^{1} \mathbf{H}$ NMR $(600$ $\left.\mathrm{MHz}, \mathrm{CDCl}_{3}\right) \delta 7.57(\mathrm{~d}, J=7.5 \mathrm{~Hz}, 2 \mathrm{H}), 7.41(\mathrm{~d}, J=7.6 \mathrm{~Hz}, 2 \mathrm{H}), 3.56(\mathrm{~s}, 2 \mathrm{H}), 2.79-2.76(\mathrm{~m}, 4 \mathrm{H}), 2.66$ - $2.63(\mathrm{~m}, 4 \mathrm{H}), 2.49(\mathrm{~s}, 3 \mathrm{H}) .{ }^{13} \mathrm{C}$ NMR $\left(151 \mathrm{MHz}, \mathrm{CDCl}_{3}\right) \delta 143.3,131.9,129.3,123.7,111.2,61.7,54.8$, 51.4, 45.0. FT-IR ( $\mathrm{cm}^{-1}$, neat, ATR) 3028, 2950, 2843, 2227, 1731, 1606, 1495, 1454, 1434, 1359, 1287, 1203, 1166, 1076, 1029, 990, 823, 740, 698, 549. HRMS (ESI-TOF) calcd for $\left(\mathrm{C}_{13} \mathrm{H}_{18} \mathrm{~N}_{3}\right)[\mathrm{M}+\mathrm{H}]^{+}$ 216.1501, found 216.1483 .

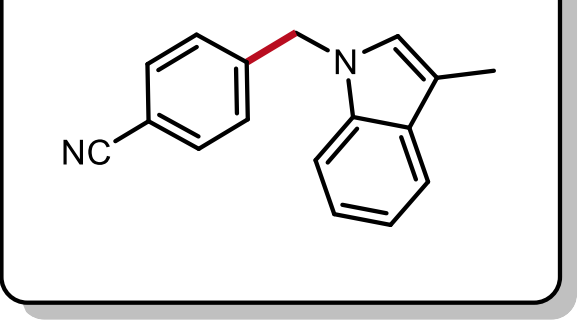

4-((3-Methyl-1H-indol-1-yl)methyl)benzonitrile (3s, $73.4 \mathrm{mg}$, 99\%) was prepared following General Procedure D with the following modification: the reaction was done in $\mathrm{N}$-methyl-2pyrrolidone $(\mathrm{NMP})(0.1 \mathrm{M})$ in place of THF $(0.1 \mathrm{M})$. This product was obtained as a white solid $\left(\mathrm{mp}=57-58^{\circ} \mathrm{C}\right) .{ }^{1} \mathrm{H}$ NMR $(500$ $\left.\mathrm{MHz}, \mathrm{CDCl}_{3}\right) \delta 7.67-7.61(\mathrm{~m}, 1 \mathrm{H}), 7.56(\mathrm{~d}, \mathrm{~J}=8.2 \mathrm{~Hz}, 2 \mathrm{H}), 7.24-7.12(\mathrm{~m}, 5 \mathrm{H}), 6.90(\mathrm{~s}, 1 \mathrm{H}), 5.31(\mathrm{~s}$, 2H), 2.39 (s, 3H). ${ }^{13} \mathrm{C}$ NMR (126 MHz, $\left.\mathrm{CDCl}_{3}\right) \delta 143.6,136.6,132.7,129.3,127.3,125.8,122.2,119.5$, 119.4, 118.8, 111.9, 111.6, 109.4, 49.5, 9.8. FT-IR (cm ${ }^{-1}$, neat, ATR): 2917, 2228, 1609, 1505, 1481, $1465,1437,1415,1387,1366,1354,1330,1259,1203,1181,1014,818,785,740,547$. HRMS (EITOF) calcd for $\left(\mathrm{C}_{17} \mathrm{H}_{14} \mathrm{~N}_{2}\right)[\mathrm{M}]^{+} 246.1157$, found 246.1160 . 


\section{Procedure for Large Scale Reaction}

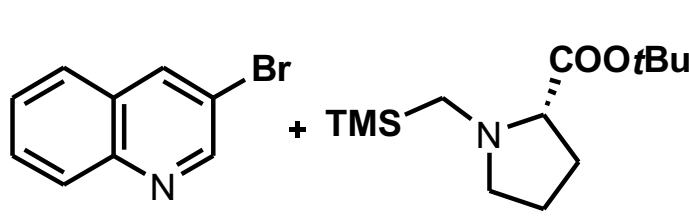

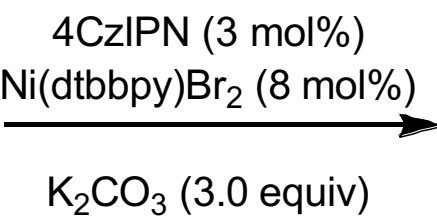

$\operatorname{THF}(0.1 \mathrm{M})$ $20 \mathrm{~h}$, blue LEDs<smiles>CCOC(=O)[C@H]1CCCN1Cc1cnc2ccccc2c1</smiles>

$3 p, 1.21 \mathrm{~g}, 69 \%$

To a $100 \mathrm{~mL}$ round bottom flask equipped with a stirrer bar was added 4CzIPN (132.9 mg, $0.25 \mathrm{mmol}$, $3 \mathrm{~mol} \%$ ), Ni(dtbbpy)Br 2 (218.7 mg, $0.67 \mathrm{mmol}, 8 \mathrm{~mol} \%$ ), 3-bromoquinoline (1.2 g, $5.6 \mathrm{mmol}, 1.0$ equiv), tert-butyl ((trimethylsilyl)methyl)-L-prolinate $(2.2 \mathrm{~g}, 8.4 \mathrm{mmol}, 1.5 \mathrm{equiv})$ and $\mathrm{K}_{2} \mathrm{CO}_{3}(2.3 \mathrm{~g}, 16.8 \mathrm{mmol}$, 3.0 equiv). The reaction vessel was sealed with a rubber septum and cooled to $0^{\circ} \mathrm{C}$ in an ice-water bath, then it was evacuated and backfilled with argon three times. After that, degassed THF $(0.1 \mathrm{M})$ was added, and the vessel was removed from the ice-water bath and further sealed with Parafilm ${ }^{\circledR}$. The vessel was then placed in a sonicator for $3 \mathrm{~min}$ and then irradiated with blue LEDs $(470 \mathrm{~nm}, \sim 10 \mathrm{~W}$, at a distance of $\sim 2 \mathrm{~cm}$ ). After $20 \mathrm{~h}$, the reaction was judged complete as monitored by TLC. The reaction mixture was poured into $50 \mathrm{~mL}$ of acetone, and the crude material was passed through a pad of Celite ${ }^{\circledR}$ and eluted with another $50 \mathrm{~mL}$ of acetone. The filtrate was concentrated under reduced pressure and purified by $\mathrm{SiO}_{2}$ column chromatography. $\mathrm{SiO}_{2}$ chromatography was performed on an automated flash chromatography system, monitoring with a UV detector at $254 \mathrm{~nm}, 280 \mathrm{~nm}$, and 200-300 nm (Threshold: 0.20 AU, signal gain 1x), and an ELSD (Threshold: $0.05 \mathrm{~V}$, Spray Temperature: $30^{\circ} \mathrm{C}$, Drift Temperature: $60{ }^{\circ} \mathrm{C}$, signal gain $1 \mathrm{x}$ ). The column used was a 40 -gram RediSep ${ }^{\circledR} \mathrm{Rf}$ silica gel disposable flash column (60 A porosity, 40-60 $\mu \mathrm{m}$ ). The column utilized a gradient elution [flow rate: $40 \mathrm{~mL} / \mathrm{min}$, hexanes (Solvent A), EtOAc (Solvent B)]: $2.2 \min (100 \%$ A), $2.8 \min (100 \%$ A to $70 \%$ A: 30\% B), 6.7 min (Hold $70 \%$ A: $30 \%$ B), $3.3 \min (70 \%$ A: $30 \%$ B to $60 \%$ A: $40 \%$ B), $16 \min (60 \%$ A: $40 \%$ B to $30 \%$ A: $70 \%$ B). The product $3 p$ was obtained as a pale-yellow oil. $(1.21 \mathrm{~g}, 69 \%)$ 


\section{SUPPORTING INFORMATION}
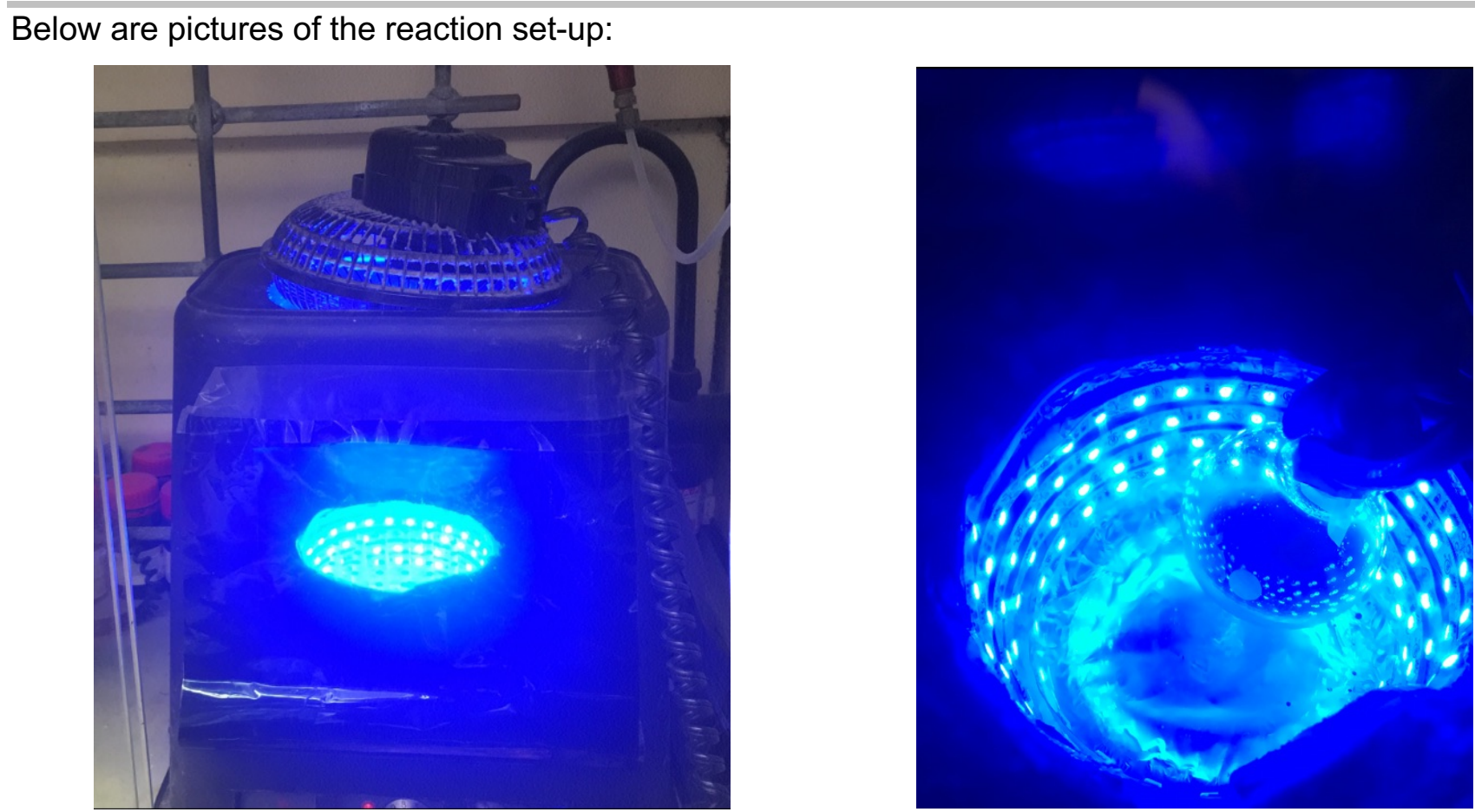

${ }^{1} \mathrm{H}$ NMR $\left(500 \mathrm{MHz}, \mathrm{CDCl}_{3}\right) \delta 8.89(\mathrm{~d}, J=2.1 \mathrm{~Hz}, 1 \mathrm{H}), 8.09(\mathrm{~s}, 1 \mathrm{H}), 8.07(\mathrm{~d}, J=8.5 \mathrm{~Hz}, 1 \mathrm{H}), 7.77(\mathrm{~d}, J=$ $8.2 \mathrm{~Hz}, 1 \mathrm{H}), 7.65$ (ddd, $J=8.5,6.8,1.4 \mathrm{~Hz}, 1 \mathrm{H}), 7.50(\mathrm{t}, J=7.5 \mathrm{~Hz}, 1 \mathrm{H}), 4.15(\mathrm{~d}, J=13.3 \mathrm{~Hz}, 1 \mathrm{H}), 3.71$ $(\mathrm{d}, J=13.3 \mathrm{~Hz}, 1 \mathrm{H}), 3.20(\mathrm{dd}, J=8.9,5.9 \mathrm{~Hz}, 1 \mathrm{H}), 3.01(\mathrm{td}, J=8.2,3.3 \mathrm{~Hz}, 1 \mathrm{H}), 2.41(\mathrm{q}, J=8.1 \mathrm{~Hz}$, 1H), $2.12-2.02(m, 1 H), 2.04-1.81(m, 2 H), 1.80-1.60(m, 1 H), 1.41(s, 9 H)$.

${ }^{13} \mathrm{C}$ NMR $\left(126 \mathrm{MHz}, \mathrm{CDCl}_{3}\right) \delta 173.2,152.1,147.7,135.5,131.9,129.3,129.1,128.1,127.7,126.6,80.8$ $65.9,56.0,53.3,29.3,28.2,23.1$.

FT-IR (cm ${ }^{-1}$, neat, ATR): 2975, 1722, 1496, 1478, 1457, 1391, 1366, 1329, 1292, 1251, 1214, 1149 , $1087,958,913,862,844,788,752,479$

HRMS (ESI-TOF) calcd for $\left(\mathrm{C}_{19} \mathrm{H}_{25} \mathrm{~N}_{2} \mathrm{O}_{2}\right)[\mathrm{M}+\mathrm{H}]^{+} 313.1925$, found 313.1916. 


\section{Streamlined synthesis of a dipeptide derivative.}

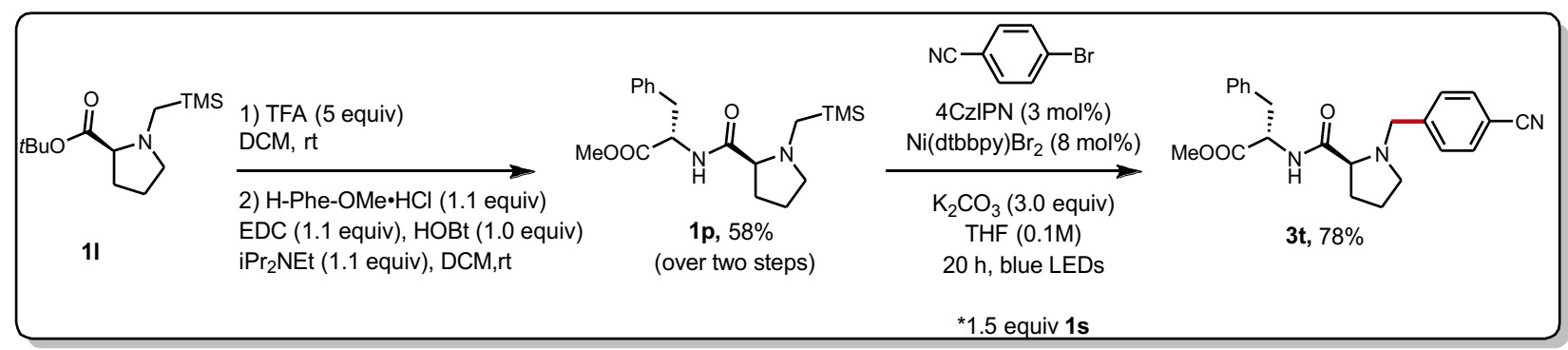

Step 1. Ester Hydrolysis: To a $25 \mathrm{~mL}$ round bottom flask was added tert-butyl ((trimethylsilyl)methyl)-Lprolinate $\left(1.000 \mathrm{~g}, 3.9 \mathrm{mmol}, 1.0\right.$ equiv). Then $\mathrm{CH}_{2} \mathrm{Cl}_{2}(10 \mathrm{~mL})$ was added, and the mixture was stirred with a magnetic stirrer. The soln was then cooled in an ice-water bath for $10 \mathrm{~min}$. After that, $\mathrm{CF}_{3} \mathrm{CO}_{2} \mathrm{H}$ (TFA) (1.5 mL, $19.5 \mathrm{mmol}, 5.0$ equiv) was added dropwise over $5 \mathrm{~min}$. Once done, the reaction mixture was warmed to rt for another $30 \mathrm{~min}$. After the reaction achieved full conversion as monitored by TLC, the reaction mixture was concentrated under reduced pressure, dried under high vacuum overnight, and used for amino acid condensation without further purification.

Step 2. Amino Acid Condensation: A modified literature procedure was used. ${ }^{[7]} \mathrm{A} 100 \mathrm{~mL}$ round bottom flask was charged with a soln of $L$-phenylalanine methyl ester hydrochloride $(925 \mathrm{mg}, 4.3 \mathrm{mmol}, 1.1$ equiv) in $\mathrm{CH}_{2} \mathrm{Cl}_{2}(50 \mathrm{~mL})$ and a magnetic stirrer. The mixture was cooled to $0{ }^{\circ} \mathrm{C}$ in an ice-water bath for 10 min. Then $i-\operatorname{Pr}_{2} \mathrm{NEt}(1.5 \mathrm{~mL}, 8.6 \mathrm{mmol}, 2.2$ equiv) was added dropwise over $5 \mathrm{~min}$. To this soln was then added all of the crude material from Step 1, hydroxybenzotriazole (HOBt: $526 \mathrm{mg}, 3.9 \mathrm{mmol}, 1.0$ equiv), and 1-(3-dimethylaminopropyl)-3-ethylcarbodiimide hydrochloride (EDC: $824 \mathrm{mg}, 4.3 \mathrm{mmol}, 1.1$ equiv). When the addition was complete, the mixture was slowly warmed to rt and kept stirring overnight. After the reaction achieved full conversion as monitored by TLC, the reaction mixture was cooled to $0{ }^{\circ} \mathrm{C}$ and quenched with $2 \mathrm{M} \mathrm{HCl}(\mathrm{aq})(5 \mathrm{~mL})$. The phases were separated and the aqueous phase was extracted with $\mathrm{CH}_{2} \mathrm{Cl}_{2}(10 \mathrm{~mL} \times 3)$. The combined organic layers were washed with brine $(10 \mathrm{~mL})$, dried $\left(\mathrm{Na}_{2} \mathrm{SO}_{4}\right)$, filtered, and concentrated under reduced pressure. The crude material was purified by $\mathrm{SiO}_{2}$ column chromatography. Flash chromatography was performed using an automated flash chromatography system, monitoring with a UV detector at $254 \mathrm{~nm}, 280 \mathrm{~nm}$, and 200-300 nm (Threshold: 
$0.20 \mathrm{AU}$, signal gain $3 \mathrm{x}$ ), and an ELSD (Threshold: $0.05 \mathrm{~V}$, Spray Temperature: $30^{\circ} \mathrm{C}$, Drift Temperature: $60{ }^{\circ} \mathrm{C}$, signal gain $3 \mathrm{x}$ ). The column used was a 40-gram RediSep ${ }^{\circledR} \mathrm{Rf}$ silica gel disposable flash columns (60 Å porosity, 40-60 $\mu \mathrm{m}$ ). The column utilized a gradient elution [flow rate: $40 \mathrm{~mL} / \mathrm{min}$, hexanes (Solvent A), EtOAc (Solvent B)]: 1 min (100\% A), $4 \min (100 \%$ A to 60\% A: 40\% B), 10 min (Hold 60\% A: 40\% B). and the product was obtained as a pale-yellow oil. (1p, $820 \mathrm{mg}, 58 \%$ over 2 steps)

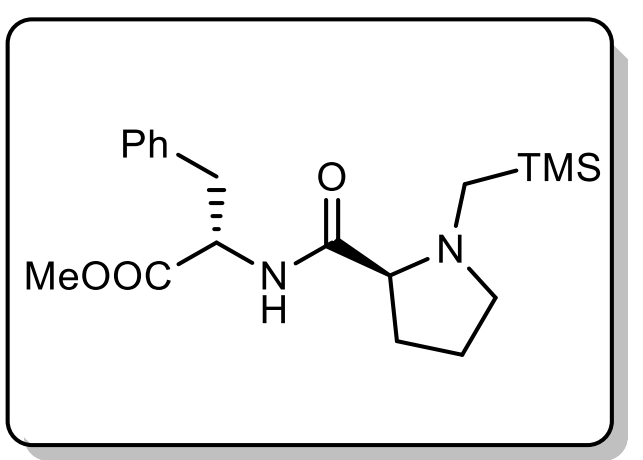

\section{Methyl ((Trimethylsilyl)methyl)-L-prolyl-L-phenylalaninate}

${ }^{1} \mathrm{H}$ NMR (1p, $\left.400 \mathrm{MHz}, \mathrm{CDCl}_{3}\right) \delta 7.68(\mathrm{~d}, \mathrm{~J}=9.2 \mathrm{~Hz}, 1 \mathrm{H}), 7.30$ $-7.16(\mathrm{~m}, 3 \mathrm{H}), 7.13-7.07(\mathrm{~m}, 2 \mathrm{H}), 4.89$ (ddd, $J=9.1,7.0,5.6$ $\mathrm{Hz}, 1 \mathrm{H}), 3.70(\mathrm{~s}, 3 \mathrm{H}), 3.14(\mathrm{dd}, J=13.9,5.6 \mathrm{~Hz}, 1 \mathrm{H}), 3.09-$ $3.00(\mathrm{~m}, 2 \mathrm{H}), 2.86(\mathrm{dd}, J=10.2,4.7 \mathrm{~Hz}, 1 \mathrm{H}), 2.24(\mathrm{~d}, J=14.1$ $\mathrm{Hz}, 1 \mathrm{H}), 2.22-2.14(\mathrm{~m}, 1 \mathrm{H}), 2.14-2.00(\mathrm{~m}, 1 \mathrm{H}), 1.79(\mathrm{~d}, \mathrm{~J}=$ 14.1 Hz, 1H), $1.74-1.66(\mathrm{~m}, 1 \mathrm{H}), 1.65-1.47(\mathrm{~m}, 2 \mathrm{H}),-0.02(\mathrm{~s}, 9 \mathrm{H}) .{ }^{13} \mathrm{C}$ NMR $\left(101 \mathrm{MHz}, \mathrm{CDCl}_{3}\right) \delta 174.6$, 172.0, 136.2, 129.3, 128.6, 127.1, 72.0, 56.2, 52.2, 47.0, 38.3, 30.3, 24.9, -1.7. FT-IR (cm ${ }^{-1}$, neat, ATR) $3348,2953,1744,1676,1498,1455,1436,1354,1312,1248,1198,1178,1129,1110,848,762,743$, 700, 609, 577, 478. HRMS (ESI-TOF) calcd for $\left(\mathrm{C}_{19} \mathrm{H}_{31} \mathrm{~N}_{2} \mathrm{O}_{3} \mathrm{Si}\right)[\mathrm{M}+\mathrm{H}]^{+} 363.2104$, found 363.2107 .

Step 3. Benzylamine Synthesis: Methyl ((trimethylsilyl)methyl)-L-prolyl-L-phenylalaninate (163.0 mg, $0.45 \mathrm{mmol}, 1.5$ equiv) and para-bromobenzonitrile ( $54.6 \mathrm{mg}, 0.3 \mathrm{mmol}, 1.0$ equiv) were subjected to General Procedure A in Section 4 for aminomethylation of aryl halides. The product was obtained as a pale-yellow oil (3t, $91.4 \mathrm{mg}, 78 \%$ ).

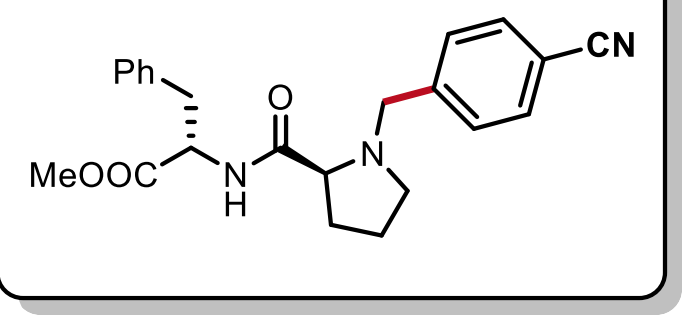

Methyl (4-Cyanobenzyl)-L-prolyl-L-phenylalaninate (3t, $91.4 \mathrm{mg}, 78 \%$ ) was prepared following General Procedure D. This product was obtained as a pale-yellow oil. ${ }^{1} \mathbf{H}$ NMR $\left(400 \mathrm{MHz}, \mathrm{CDCl}_{3}\right) \delta 7.77(\mathrm{~d}, J=9.0 \mathrm{~Hz}, 1 \mathrm{H}), 7.53(\mathrm{dd}, J=$ 8.3, 2.0 Hz, 2H), $7.34-7.22(\mathrm{~m}, 4 \mathrm{H}), 7.13-7.09(\mathrm{~m}, 3 \mathrm{H})$, 
$4.92(\mathrm{dt}, J=9.0,5.7 \mathrm{~Hz}, 1 \mathrm{H}), 3.95(\mathrm{~d}, J=13.1 \mathrm{~Hz}, 1 \mathrm{H}), 3.72(\mathrm{~s}, 3 \mathrm{H}), 3.36(\mathrm{~d}, J=13.3 \mathrm{~Hz}, 1 \mathrm{H}), 3.28-$ $3.04(\mathrm{~m}, 3 \mathrm{H}), 2.82(\mathrm{tt}, J=7.4,1.6 \mathrm{~Hz}, 1 \mathrm{H}), 2.27-2.12(\mathrm{~m}, 2 \mathrm{H}), 1.87-1.70(\mathrm{~m}, 2 \mathrm{H}), 1.69-1.55(\mathrm{~m}, 1 \mathrm{H})$.

${ }^{13} \mathrm{C}$ NMR $\left(101 \mathrm{MHz}, \mathrm{CDCl}_{3}\right) \delta$ 173.9, 172.1, 144.1, 135.9, 132.4, 129.5, 129.5, 128.7, 127.3, 119.0, 111.1, 67.6, 59.4, 53.6, 52.4, 52.0, 38.1, 30.6, 24.3. FT-IR ( $\mathrm{cm}^{-1}$, neat, ATR) 3349, 2952, 2228, 1742, 1673 , $1608,1506,1440,1414,1356,1216,1202,1179,1127,1080,1020,854,821,745,702$, 550. HRMS (ESI-TOF) calcd for $\left(\mathrm{C}_{23} \mathrm{H}_{26} \mathrm{~N}_{3} \mathrm{O}_{3}\right)[\mathrm{M}+\mathrm{H}]^{+} 392.1974$, found 392.1981. 


\subsection{Reaction carried out with secondary alkylamine 4}

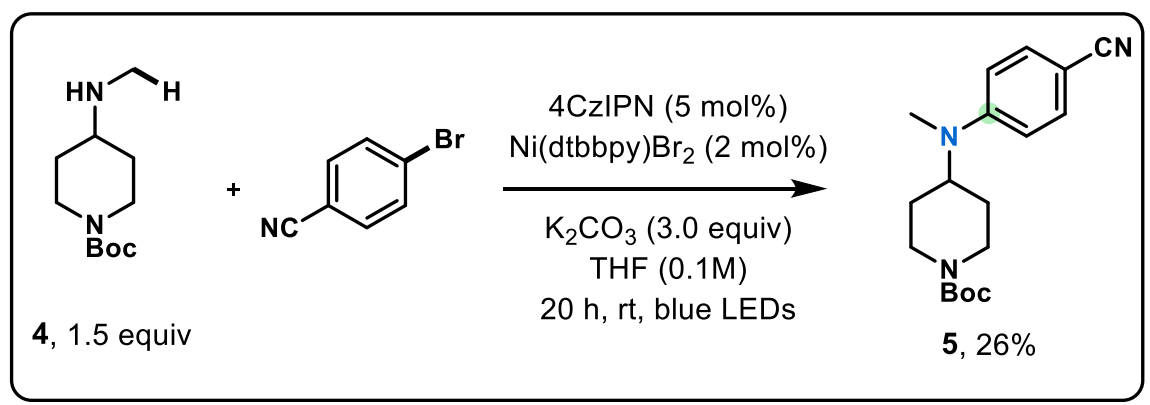

To an $8 \mathrm{~mL}$ reaction vial equipped with a stirrer bar was added $4 \mathrm{CzIPN}(11.8 \mathrm{mg}, 5 \mathrm{~mol} \%)$, Ni(dtbbpy)Br 2 (2.9 mg, $2 \mathrm{~mol} \%$ ), para-bromobenzonitrile (54.6 mg, $0.3 \mathrm{mmol}, 1.0$ equiv), amine 4 (96.4 mg, $0.45 \mathrm{mmol}$, 1.5 equiv) and $\mathrm{K}_{2} \mathrm{CO}_{3}(124.4 \mathrm{mg}, 0.9 \mathrm{mmol}, 3.0$ equiv). The reaction vessel was sealed with a cap containing a TFE-lined silicone septum and cooled to $0{ }^{\circ} \mathrm{C}$ in an ice-water bath. Then it was evacuated and backfilled with argon three times. After that, degassed THF $(3 \mathrm{~mL})$ was added, and the vessel was removed from the ice-water bath and further sealed with Parafilm ${ }^{\circledR}$. The vessel was then placed in a sonicator for $3 \mathrm{~min}$ and irradiated with blue LEDs $(470 \mathrm{~nm}, \sim 10 \mathrm{~W}$, at a distance of $\sim 2 \mathrm{~cm})$. After completion, $5 \mathrm{~mL}$ of acetone was added, and the crude material was passed through a pad of Celite ${ }^{\circledR}$ and eluted with another $5 \mathrm{~mL}$ of acetone. The filtrate was concentrated under reduced pressure and purified by $\mathrm{SiO}_{2}$ column chromatography. $\mathrm{SiO}_{2}$ chromatography was performed on an automated flash chromatography system, monitoring with a UV detector at $254 \mathrm{~nm}, 280 \mathrm{~nm}$, and 200-300 nm (Threshold: $0.20 \mathrm{AU}$, signal gain 5x), and an ELSD (Threshold: $0.05 \mathrm{~V}$, Spray Temperature: $30^{\circ} \mathrm{C}$, Drift Temperature: $60{ }^{\circ} \mathrm{C}$, signal gain $5 \mathrm{x}$ ). The column used was a 24 -gram RediSep ${ }^{\circledR} \mathrm{R}_{\mathrm{f}}$ silica gel disposable flash column (60 Å porosity, 40-60 $\mu \mathrm{m}$ ). The column utilized a gradient elution [flow rate: $40 \mathrm{~mL} / \mathrm{min}$, hexanes (Solvent A), EtOAc (Solvent B)]: $3 \min (100 \%$ A to $90 \%$ A: 10\% B), $14 \min (90 \%$ A: $10 \%$ B to $70 \%$ A: $30 \%$ B), 3 min (Hold $70 \%$ A: $30 \%$ B). This product 5 was obtained as a pale-yellow oil $(24.6 \mathrm{mg}, 26 \%)$

${ }^{1} \mathrm{H}$ NMR $\left(600 \mathrm{MHz}, \mathrm{CDCl}_{3}\right) \delta 7.47(\mathrm{~d}, J=9.0 \mathrm{~Hz}, 2 \mathrm{H}), 6.72(\mathrm{~d}, J=9.1 \mathrm{~Hz}, 2 \mathrm{H}), 4.27(\mathrm{br}, 2 \mathrm{H}), 3.79$ (tt, $J$ $=11.1,4.4 \mathrm{~Hz}, 1 \mathrm{H}), 2.83(\mathrm{~s}, 3 \mathrm{H}), 2.79(\mathrm{~s}, 2 \mathrm{H}), 1.73-1.65(\mathrm{~m}, 4 \mathrm{H}), 1.48(\mathrm{~s}, 9 \mathrm{H})$. 
${ }^{13} \mathrm{C}$ NMR $\left(151 \mathrm{MHz}, \mathrm{CDCl}_{3}\right) \delta$ 154.8, 152.2, 133.8, 120.7, 112.2, 98.1, 80.1, 56.2, 43.6, 31.4, 29.3, 28.6.

FT-IR $\left(\mathrm{cm}^{-1}\right.$, neat, ATR): 2929, 2213, 1746, 1690, 1606, 1519, 1423, 1364, 1321, 1274, 1241, 1171 , 1138, 1096, 1014, 990, 867, 818, 698, 544.

HRMS (ESI-TOF) calcd for $\left(\mathrm{C}_{18} \mathrm{H}_{26} \mathrm{~N}_{3} \mathrm{O}_{2}\right)[\mathrm{M}+\mathrm{H}]^{+} 316.2038$, found 316.2025.

\subsection{Investigation into the involvement of aza-brook rearrangement}

The reaction is carried out following General Procedure $\mathbf{C}$. After the reaction is complete, the reaction mixture is allowed to settle for $2 \mathrm{~h}$. The clear solution is then carefully transferred to a $20 \mathrm{~mL}$ vial by a Pasteur pipette. The solution is concentrated under reduced pressure and used directly for the ${ }^{29} \mathrm{Si}$ NMR study.

$$
{ }^{29} \mathrm{Si} \mathrm{NMR}\left(\mathrm{CDCl}_{3}\right)
$$

Pure ${ }^{29}$ Si NMR of $1 i$

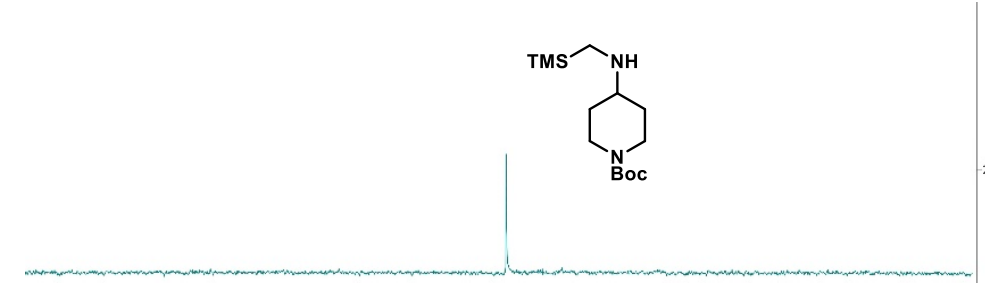

Crude reaction mixture

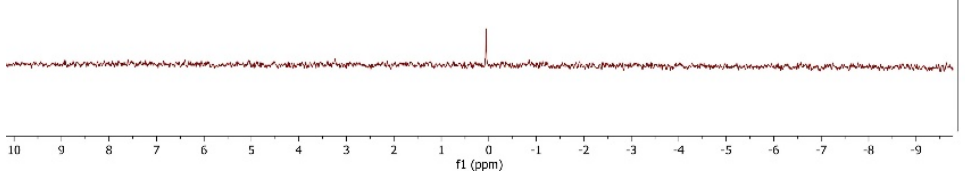

Figure S1. ${ }^{29} \mathrm{Si} \mathrm{NMR}$ studies on the reaction mixture.

As indicated in the ${ }^{29} \mathrm{Si}$ NMR spectra of the crude reaction mixture, no silyl containing species is observed other than the starting material $1 \mathbf{i}$.

\subsection{Stoichiometric experiment with Ni-aryl halide complex}




\section{Synthesis of $\mathrm{Ni}(\mathrm{II})$-aryl halide complex 10}

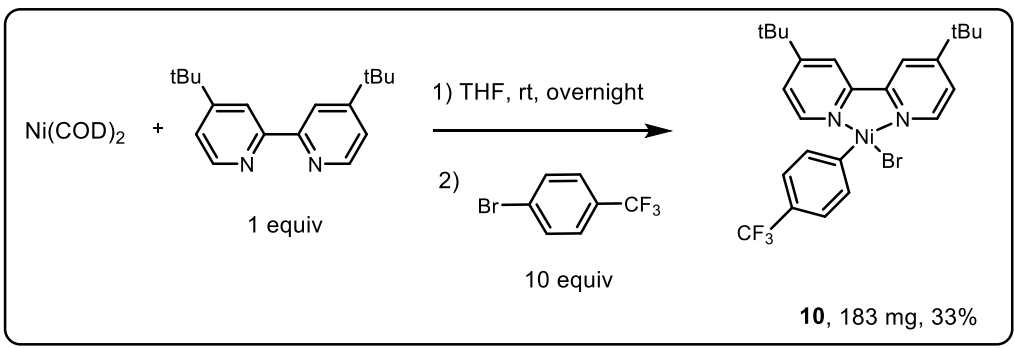

The synthesis is adapted from a literature procedure ${ }^{[8]} \mathrm{In}$ a nitrogen-filled glove box, a $40 \mathrm{~mL}$ vial with a stirring bar is charged with $\mathrm{Ni}(\mathrm{COD})_{2}(276 \mathrm{mg}, 1.0 \mathrm{mmol}, 1.0$ equiv), 4,4'-di-tert-butyl-2,2'-bipyridine (268 $\mathrm{mg}, 1.0 \mathrm{mmol}, 1.0$ equiv) and dry THF (10 mL). The reaction vessel is sealed with a cap containing a TFE-lined silicone septum and then covered by aluminum foil to avoid light. The mixture turns a dark purple color and is allowed to stir overnight at rt. para-Bromobenzotrifluoride $(2.8 \mathrm{~mL}, 20 \mathrm{mmol}, 10.0$ equiv) is then added, and the reaction mixture is stirred for another $3 \mathrm{~h}$. Dry pentane $(60 \mathrm{~mL})$ was added to the reaction mixture and the reaction mixture was then filtered. The resulting precipitate was washed with pentane $(3 \times 10 \mathrm{~mL})$ and dried under vacum to afford $\mathrm{Ni}(\mathrm{II})$ complex 10 as a yellow solid (183 $\mathrm{mg}$, $33 \%)$. Characterization is in agreement with the literature.

\section{Stoichiometric experiment with $\alpha$-silylamine 1i}

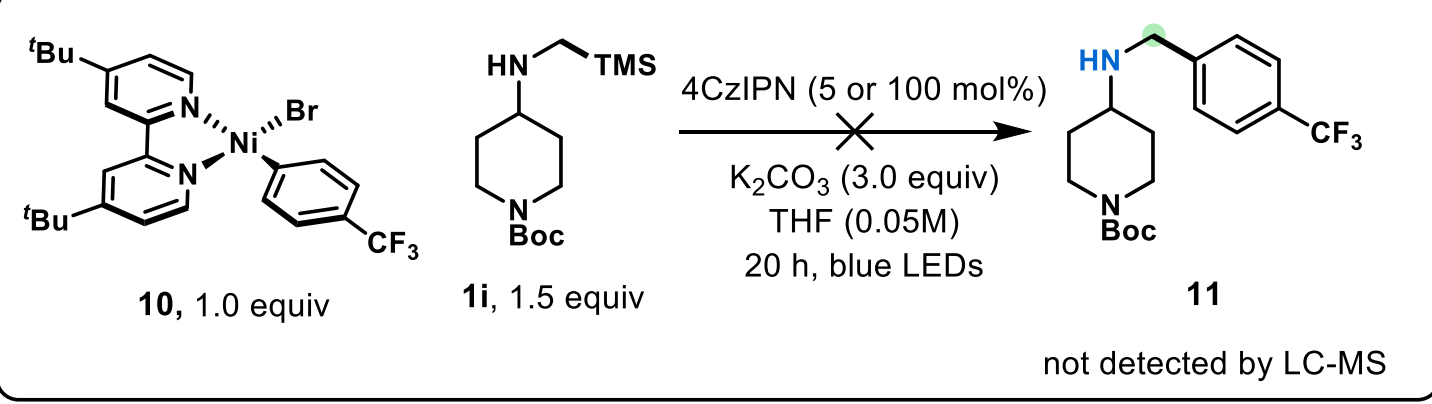

To a $4 \mathrm{~mL}$ reaction vial equipped with a stirrer bar was added $\mathrm{Ni}(\mathrm{II})$ complex 10 (27.6 mg, 0.05mmol, 1.0 equiv), 4CzIPN (39.5 mg, $0.05 \mathrm{mmol}, 1.0$ equiv or $2.0 \mathrm{mg}, 0.0025 \mathrm{mmol}, 0.05$ equiv), $\alpha$-silylamine $1 \mathrm{i}$ (21.5 mg, 0.075 mmol, 1.5 equiv), and $\mathrm{K}_{2} \mathrm{CO}_{3}$ (20.7 mg, $0.15 \mathrm{mmol}, 3.0$ equiv). The reaction vessel was sealed with a cap containing a TFE-lined silicone septum and cooled to $0{ }^{\circ} \mathrm{C}$ in an ice-water bath. Then 
it was evacuated and backfilled with argon three times. After that, degassed THF (1 mL) was added, and the vessel was removed from the ice-water bath and further sealed with Parafilm ${ }^{\circledR}$. The vessel was then placed in a sonicator for $3 \mathrm{~min}$ and irradiated with blue LEDs $(470 \mathrm{~nm}, \sim 10 \mathrm{~W}$, at a distance of $\sim 2 \mathrm{~cm})$ for $20 \mathrm{~h}$. The crude reaction was analyzed by LC-MS and showed no evidence for the presence of amine 11 when compared with product standard.

\subsection{Control experiments with catalytic amount of $\mathrm{Ni}$ complex}

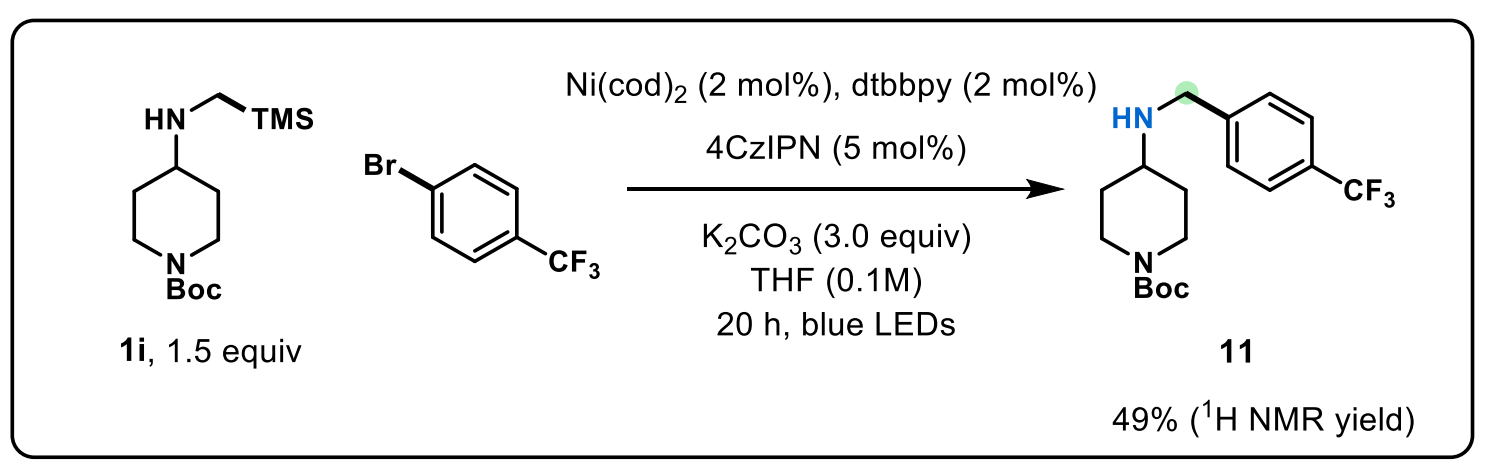

To a $4 \mathrm{~mL}$ reaction vial equipped with a stirrer bar was added bis(1,5-cyclooctadiene)nickel(0) $(0.6 \mathrm{mg}$, $0.002 \mathrm{mmol}, 0.02$ equiv), 4,4'-di-tert-butyl-2,2'-dipyridyl ( $0.5 \mathrm{mg}, 0.002 \mathrm{mmol}, 0.02$ equiv), 4CzIPN (3.9 $\mathrm{mg}, 0.005 \mathrm{mmol}, 0.05$ equiv), $\alpha$-silylamine $1 \mathrm{i}$ (43.0 mg, $0.15 \mathrm{mmol}, 1.5$ equiv), 4-bromobenzotrifluoride (22.5 mg, 0.10 mmol, 1.0 equiv) and $\mathrm{K}_{2} \mathrm{CO}_{3}(41.5 \mathrm{mg}, 0.30 \mathrm{mmol}, 3.0$ equiv). The reaction vessel was sealed with a cap containing a TFE-lined silicone septum and cooled to $0{ }^{\circ} \mathrm{C}$ in an ice-water bath. Then it was evacuated and backfilled with argon three times. After that, degassed THF (1 mL) was added, and the vessel was removed from the ice-water bath and further sealed with Parafilm ${ }^{\circledR}$. The vessel was then placed in a sonicator for $3 \mathrm{~min}$ and irradiated with blue LEDs $(470 \mathrm{~nm}, \sim 10 \mathrm{~W}$, at a distance of $\sim 2 \mathrm{~cm})$ for $20 \mathrm{~h}$. The crude mixture was filtered by Celite ${ }^{\circledR} 545$. Then the crude reaction was evaluated by ${ }^{1} \mathrm{H}$ NMR to determine product yield against 1,3,5-triisopropylbenzene. 


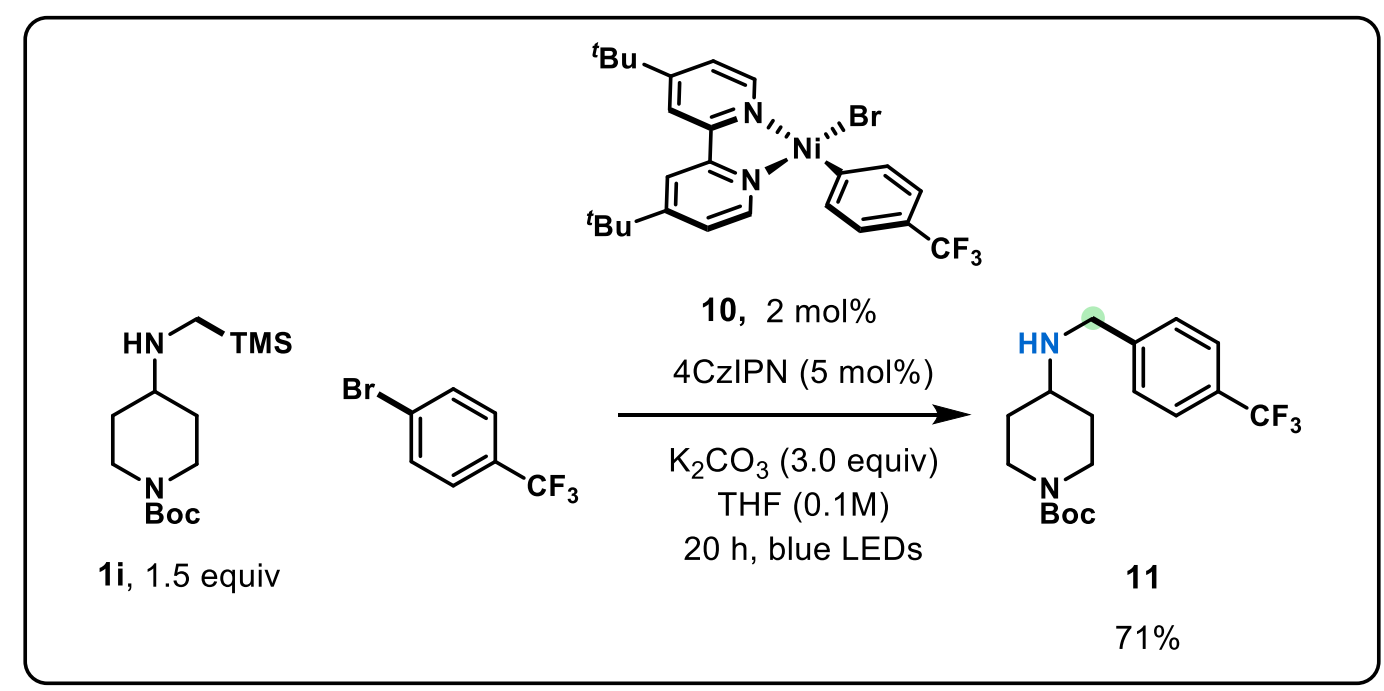

To a $4 \mathrm{~mL}$ reaction vial equipped with a stirrer bar was added $\mathrm{Ni}(\mathrm{II})$ complex 10 (1.1 $\mathrm{mg}, 0.002 \mathrm{mmol}$, 0.02 equiv), 4CzIPN (3.9 mg, 0.005 mmol, 0.05 equiv), $\alpha$-silylamine $1 \mathrm{i}$ (43.0 mg, 0.15 mmol, 1.5 equiv), 4-bromobenzotrifluoride (22.5 mg, $0.10 \mathrm{mmol}, 1.0$ equiv) and $\mathrm{K}_{2} \mathrm{CO}_{3}(41.5 \mathrm{mg}, 0.30 \mathrm{mmol}, 3.0$ equiv). The reaction vessel was sealed with a cap containing a TFE-lined silicone septum and cooled to $0{ }^{\circ} \mathrm{C}$ in an ice-water bath. Then it was evacuated and backfilled with argon three times. After that, degassed THF (1 mL) was added, and the vessel was removed from the ice-water bath and further sealed with Parafilm ${ }^{\circledR}$. The vessel was then placed in a sonicator for $3 \mathrm{~min}$ and irradiated with blue LEDs (470 $\mathrm{nm}$, $\sim 10 \mathrm{~W}$, at a distance of $\sim 2 \mathrm{~cm}$ ) for $20 \mathrm{~h}$. When the reaction was done, $5 \mathrm{~mL}$ of acetone was added, and the crude material was passed through a pad of Celite ${ }^{\circledR}$ and eluted with another $5 \mathrm{~mL}$ of acetone. The filtrate was concentrated under reduced pressure and purified by $\mathrm{SiO}_{2}$ column chromatography. $\mathrm{SiO}_{2}$ chromatography was performed on an automated flash chromatography system, monitoring with a UV detector at $254 \mathrm{~nm}, 280 \mathrm{~nm}$, and 200-300 nm (Threshold: $0.20 \mathrm{AU}$, signal gain 5x), and an ELSD (Threshold: $0.05 \mathrm{~V}$, Spray Temperature: $30{ }^{\circ} \mathrm{C}$, Drift Temperature: $60^{\circ} \mathrm{C}$, signal gain $5 \mathrm{x}$ ). The column used was a 24-gram RediSep® Rf silica gel disposable flash column (60 A porosity, $40-60 \mu \mathrm{m}$ ). Unless otherwise noted, the column utilized a gradient elution [flow rate: $40 \mathrm{~mL} / \mathrm{min}$, Dichloromethane (Solvent A), Methanol (Solvent B)]: $1 \mathrm{~min}(100 \%$ A), $11 \min (100 \%$ A to $75 \%$ A: 25\% B). Product 11 was isolated as a pale-yellow oil $(25.6 \mathrm{mg}, 71 \%)$. 
${ }^{1} \mathrm{H}$ NMR $\left(600 \mathrm{MHz}, \mathrm{CDCl}_{3}\right) \delta 7.57(\mathrm{~d}, J=8.3 \mathrm{~Hz}, 2 \mathrm{H}), 7.45(\mathrm{~d}, J=7.9 \mathrm{~Hz}, 2 \mathrm{H}), 4.01(\mathrm{br}, 2 \mathrm{H}), 3.88$ (s, 2H), $2.82-2.79(\mathrm{~m}, 2 \mathrm{H}), 2.69-2.61(\mathrm{~m}, 1 \mathrm{H}), 1.88-1.83(\mathrm{~m}, 2 \mathrm{H}), 1.45(\mathrm{~s}, 9 \mathrm{H}), 1.34(\mathrm{br}, 1 \mathrm{H}), 1.32-$ $1.24(\mathrm{~m}, 2 \mathrm{H})$.

${ }^{13} \mathrm{C}$ NMR $\left(151 \mathrm{MHz}, \mathrm{CDCl}_{3}\right) \delta 154.96,144.91,129.71,129.50,129.28,129.07,128.33,127.07,125.53$, $125.50,125.48,125.45,125.27,123.47,121.67,79.59,54.48,50.44,42.42,32.65,28.58$.

${ }^{19} \mathrm{~F}$ NMR $\left(376 \mathrm{MHz}, \mathrm{CDCl}_{3}\right) \delta-62.4$.

FT-IR (cm ${ }^{-1}$, neat, ATR): 2977, 2933, 2854, 1686, 1619, 1449, 1420, 1366, 1325, 1276, 1236, 1163 , 1123, 1066, 1018, 937, 867, 825, 769, 592.

HRMS (ESI-TOF) calcd for $\left(\mathrm{C}_{18} \mathrm{H}_{26} \mathrm{~F}_{3} \mathrm{~N}_{2} \mathrm{O}_{2}\right)[\mathrm{M}+\mathrm{H}]^{+}$359.1946, found 359.1944.

\subsection{Stern-Volmer Quenching Studies}

Fluorescence measurements were obtained using septa-capped UV-Quartz cuvettes (10 mm pathlength). Excitation was performed at $370 \mathrm{~nm}$; fluorescence spectra were obtained from 400-700 $\mathrm{nm}$. In a nitrogen filled glovebox, the following stock solutions were prepared:

A) Photocatalyst solution (0.0002 M): To an oven dried scintillation vial equipped with a stir bar was added 4CzIPN (1.76 mg, $\left.2.23 \times 10^{-3} \mathrm{mmol}\right)$. This was diluted with $10.8 \mathrm{~mL}$ of THF and stirred until completely dissolved, producing a $2.06 \times 10^{-4} \mathrm{M}$ solution of $4 \mathrm{CzIPN}$.

B) Organosilane solution $(0.004 \mathrm{M})$ : To an oven dried scintillation vial equipped with a stir bar, was added $\alpha$-silylamine $1 \mathrm{i}\left(12.38 \mathrm{mg}, 4.32 \times 10^{-2} \mathrm{mmol}\right)$. This was diluted in $10.8 \mathrm{~mL}$ of THF and stirred until completely dissolved, producing a colorless, $4.00 \times 10^{-3} \mathrm{M}$ solution of $1 \mathrm{i}$.

Following preparation, the solutions were allocated to the cuvettes and fluorescence quenching was determined with $\alpha$-silylamine $1 \mathrm{i}$. The $\mathrm{I}_{0} / \mathrm{l}$ value of each sample were calculated from the average of three scans per data point. Linear regression of $I_{0} / l$ against concentration was carried out to yield the 
Stern-Volmer quenching rate constant (Ksv). The following Stern-Volmer plot for luminescence quenching of $4 \mathrm{CzIPN}\left(1.96 \times 10^{-5} \mathrm{M}\right.$ in degassed THF) by the organosilane quencher was obtained. These results highlight that the excited catalyst is efficiently quenched by the organosilane with a Stern-Volmer quenching rate constant of $734.6 \mathrm{M}^{-1}$.

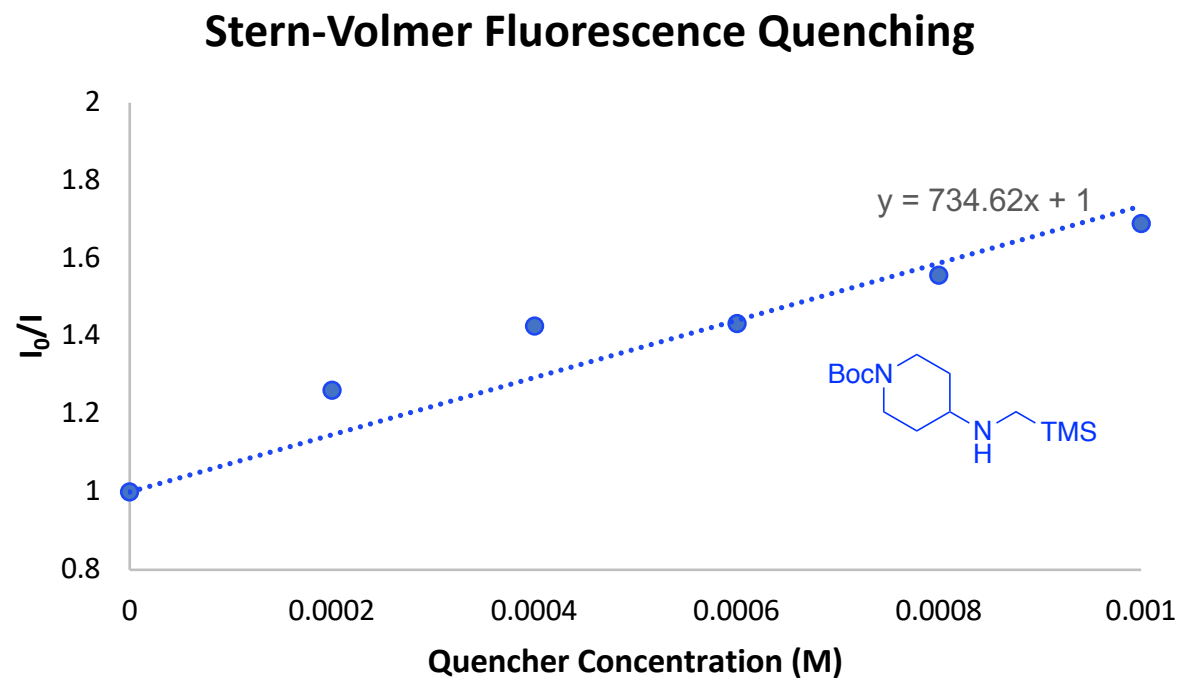

Figure S2. Stern-Volmer plot for luminescence quenching of $4 \mathrm{CzIPN}\left(1.96 \times 10^{-5} \mathrm{M}\right.$ in degassed THF) by organosilane $1 \mathbf{i}, \lambda_{\text {exc. }}=370 \mathrm{~nm}, \lambda_{\mathrm{em}} .=535 \mathrm{~nm}, \mathrm{~K}_{\mathrm{sv}}=$ SternVolmer constant. 


\section{Cyclic Voltammogram of Representative Amines}

Cyclic voltametry were conducted on a $\mathrm{CH}$ Instruments 600E Series Electrochemical Analyzer. Voltammogram were recorded using a glassy carbon working electrode, a platinum counter electrode and a silver reference electrode in a $0.1 \mathrm{M}\left[{ }^{n} \mathrm{bu}_{4} \mathrm{~N}\right]\left[\mathrm{ClO}_{4}\right]$ supporting electrolyte $\mathrm{MeCN}$ solution, with ferrocene as an internal reference $\left(E_{1 / 2}^{0}=+0.40 \mathrm{~V}\right.$ vs SCE). Cyclic voltammograms were recorded with a step potential of $0.001 \mathrm{~V}$ at a scan rate of $0.2 \mathrm{~V} / \mathrm{s}$.

$$
\begin{gathered}
\mathrm{E}_{1 / 2}(\mathrm{~V} \text { vs SCE }) \\
\alpha \text {-silylamines }
\end{gathered}
$$<smiles>COC(=O)C(Cc1ccccc1)NCCS(=O)(=O)c1ccccc1</smiles>

$1 \mathrm{a},+1.02 \mathrm{~V}$

secondary amines<smiles>CC(=O)OCc1ccccc1</smiles>

$2 \mathrm{a},+1.57 \mathrm{~V}$

tertiary amines<smiles>CCCCOC(=O)[C@@H]1CCCN1Cc1ccc(C#N)cc1</smiles>

$3 a,+1.16 \mathrm{~V}$<smiles>CC(C)(C)N1CCC(CNS(C)(=O)=O)CC1</smiles>

$1 \mathrm{i},+1.02 \mathrm{~V}$<smiles>CC(=O)C(Cc1ccccc1)NCc1cncnc1</smiles>

$2 \mathrm{~g},+1.54 \mathrm{~V}$<smiles>N#Cc1ccc(CN2CCCCC2)cc1</smiles>

$+1.37 \mathrm{~V}^{[9]}$<smiles>CCCCOC(=O)[C@@H]1CCCN1CS(C)(=O)=O</smiles>

$10,+0.74 \mathrm{~V}$<smiles>COc1ccccc1CNC(Cc1ccccc1)C(C)=O</smiles>

$2 \mathrm{e},+1.38 \mathrm{~V}$<smiles>CCN(CC)Cc1ccc(C#N)cc1</smiles>

$+1.25 \mathrm{~V}[9]$

Figure S3. $E_{1 / 2}(V$ vs SCE) of representative amines.

Electrochemical analysis shows that comparing with the $\alpha$-silylamine starting materials, the corresponding benzyl amines have significantly higher oxidative potentials (0.2-0.6V higher). This means that the oxidation of $\alpha$-silylamine is significantly thermodynamically more favorable. Although some of the benzyl amines are susceptible to oxidation by the excited state of $4 \mathrm{CzIPN}\left(+1.35 \mathrm{~V}\right.$ vs $\mathrm{SCE}$ in $\left.\mathrm{CH}_{3} \mathrm{CN}\right)$, the rate of back electron transfer may significantly increase without a cleavable $\alpha$-silyl group, which leads to no further reaction happens on the products. 

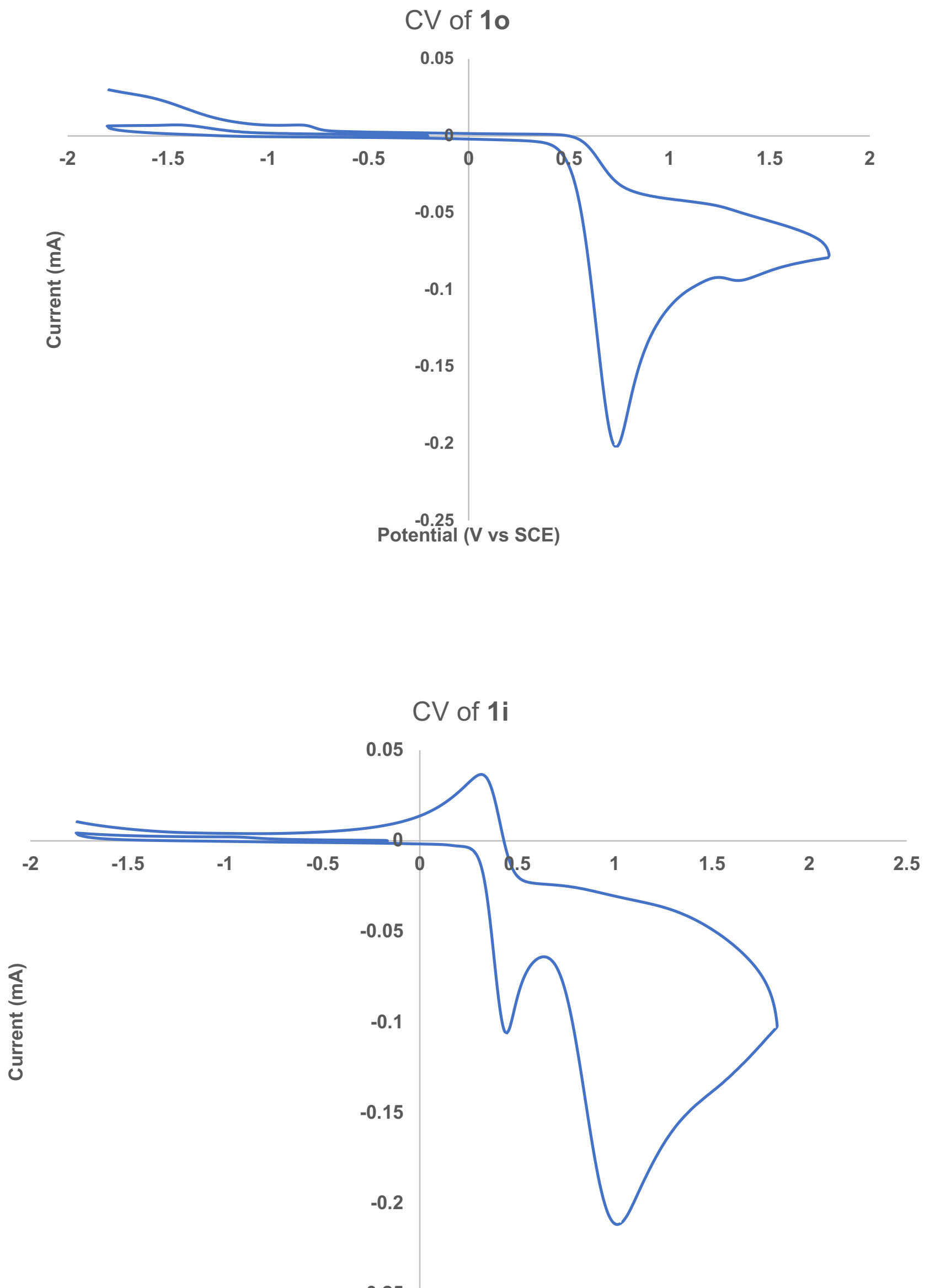

$-0.25$ 

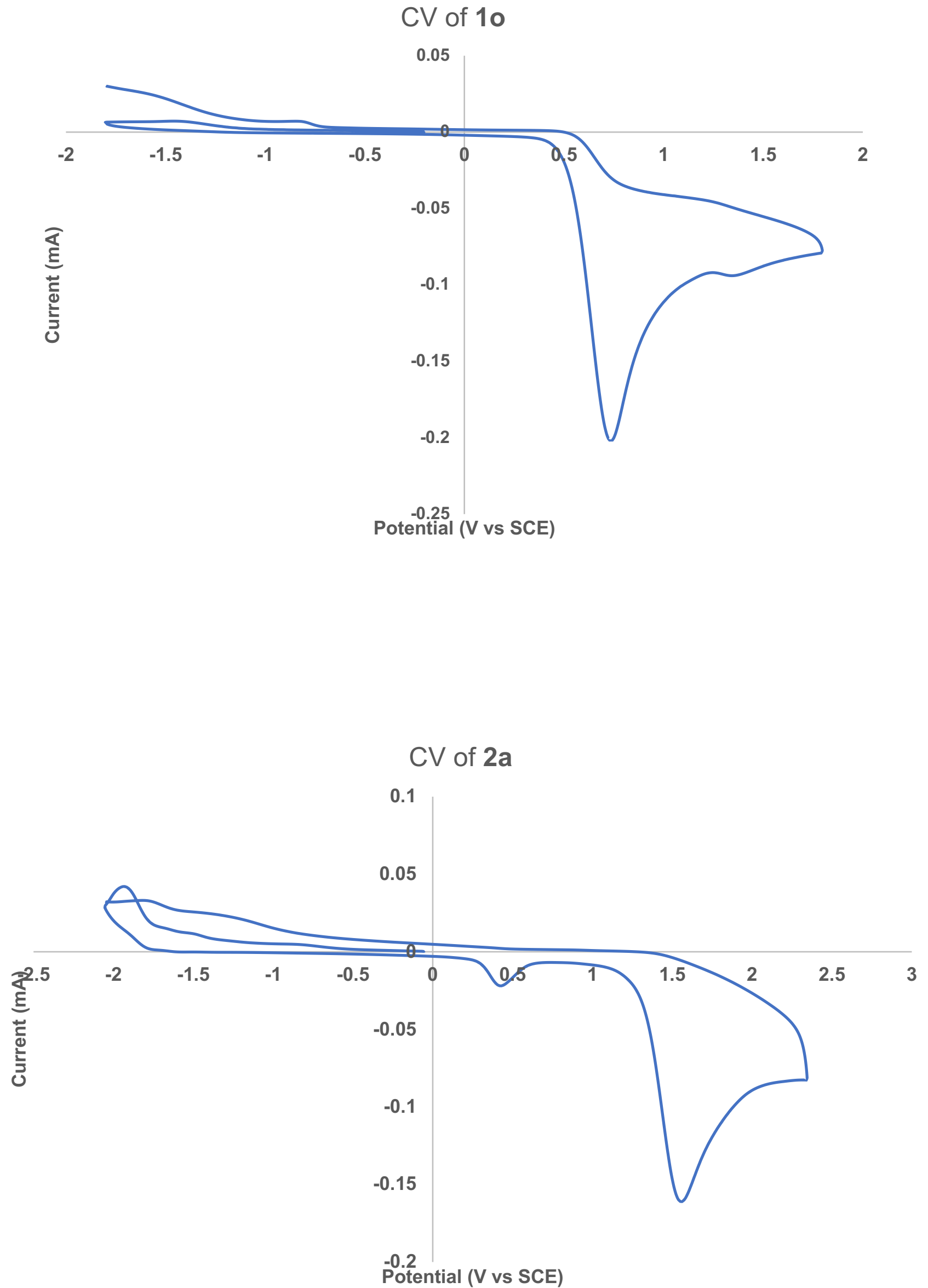

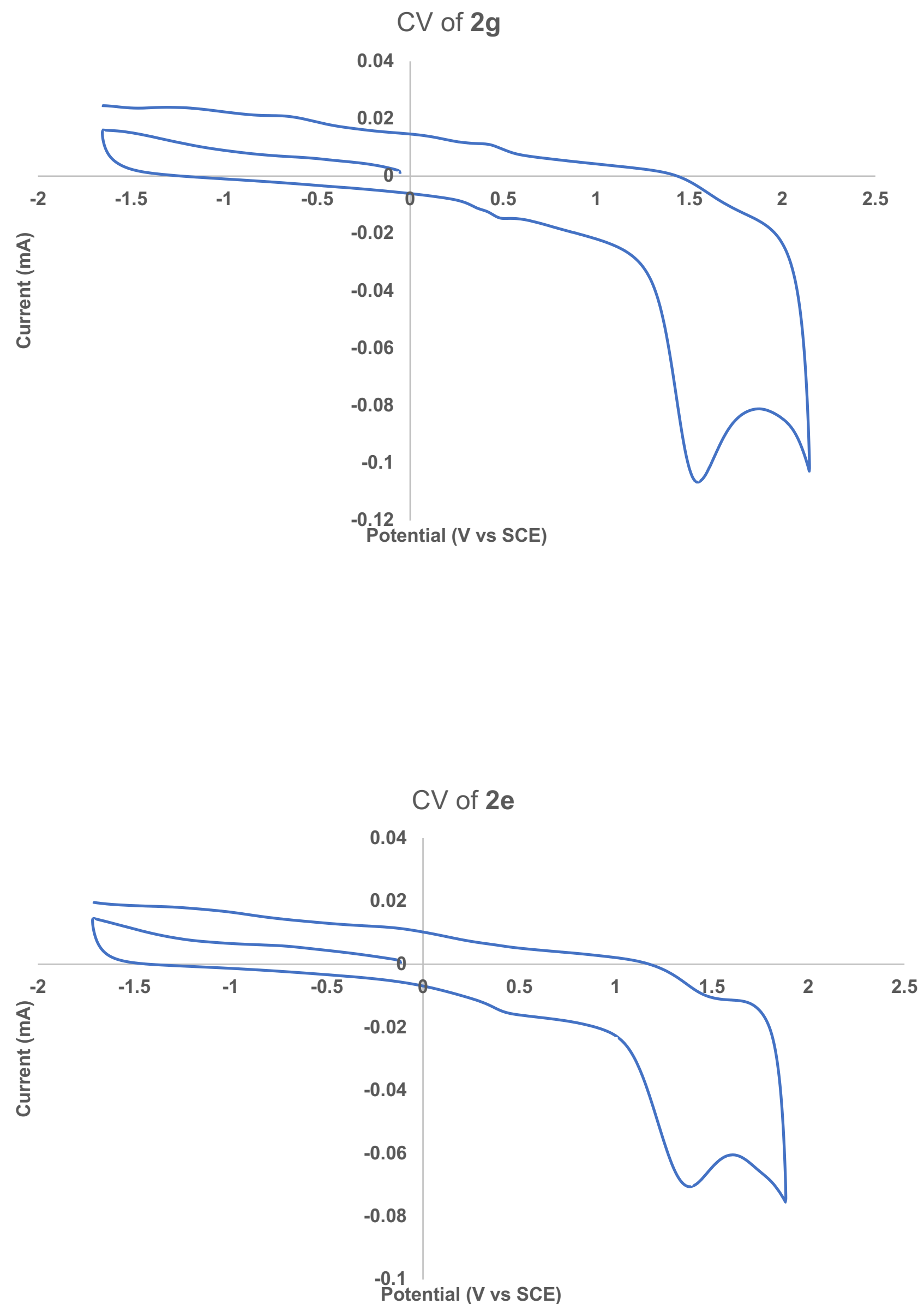

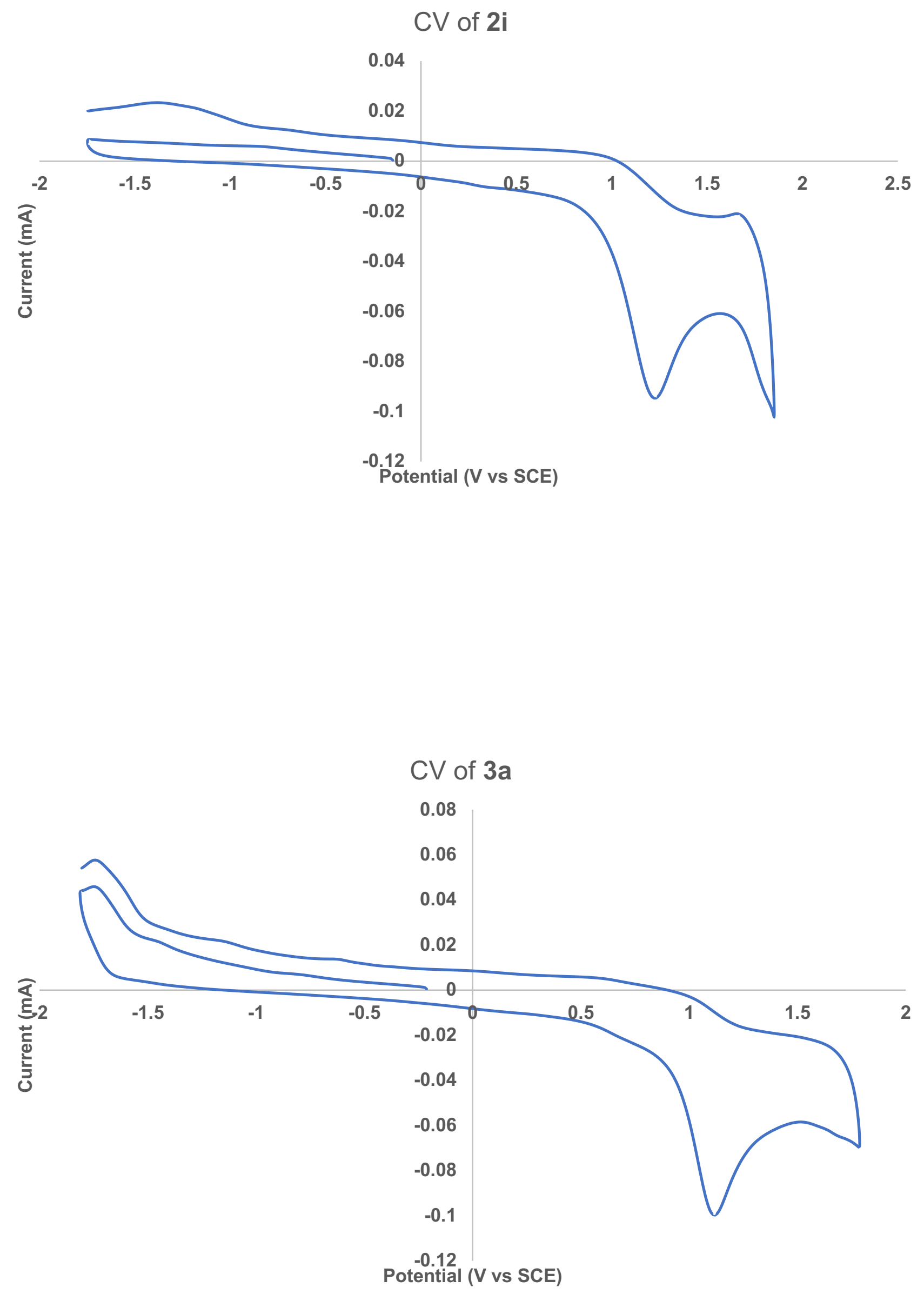


\section{Crystallographic Data}

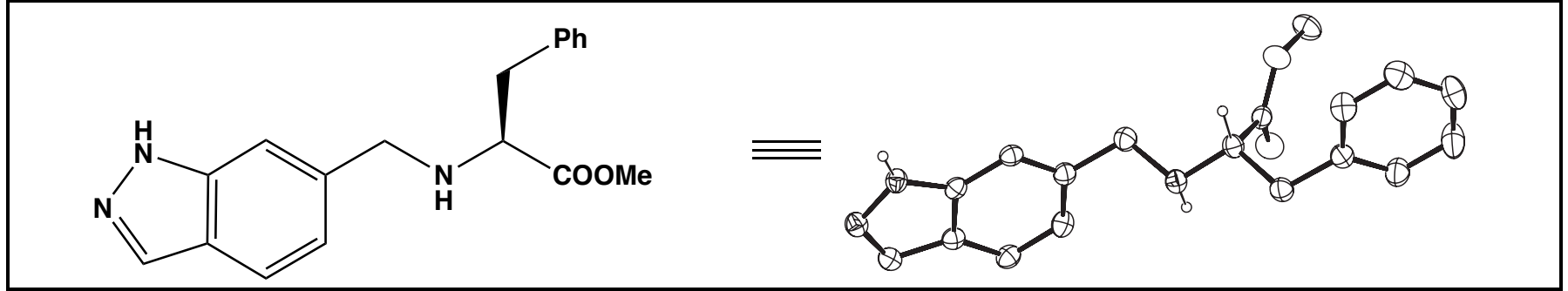

Crystal of compound $\mathbf{2} \mathbf{i}$ was prepared in a solvent mixture of methanol and chloroform $(\sim v / v=10: 1)$. White solid of $2 \mathbf{i}(\sim 50 \mathrm{mg})$ was dissolved in nearly boiled methanol ( $\sim 5 \mathrm{~mL})$ by adding chloroform dropwise until all material was dissolved. Then the sample was carefully sealed and cooled to room temperature. Once cooled, the sample was placed into a $-20^{\circ} \mathrm{C}$ environment overnight. The crystal was obtained after cooling overnight.

Compound $2 \mathbf{i}, \mathrm{C}_{18} \mathrm{H}_{19} \mathrm{~N}_{3} \mathrm{O}_{2}$, crystallizes in the monoclinic space group $\mathrm{P} 2_{1}$ (systematic absences $0 \mathrm{k} 0$ : $k=$ odd) with $a=8.9278(2) \AA, b=5.45340(10) \AA, c=16.5048(3) \AA, \beta=99.231(2)^{\circ}, V=793.16(3) \AA^{3}, Z=2$, and $d_{\text {calc }}=1.295 \mathrm{~g} / \mathrm{cm}^{3}$. X-ray intensity data were collected on a Rigaku XtaLAB Synergy-S diffractometer ${ }^{[10]}$ equippped with an HPC area detector (HyPix-6000HE) and employing confocal multilayer opticmonochromated Cu-Ka radiation $(\lambda=1.54184 \AA)$ at a temperature of $100.00 \mathrm{~K}$. Preliminary indexing was performed from a series of sixty $0.5^{\circ}$ rotation frames with exposures of $1 \mathrm{sec}$. at $\theta= \pm 48.77^{\circ}$ and $4 \mathrm{sec}$. at $\theta=105.75^{\circ}$. A total of 5988 frames (41 runs) were collected employing $\omega$ scans with a crystal to detector distance of $31.6 \mathrm{~mm}$, rotation widths of $0.5^{\circ}$ and exposures of $1 \mathrm{sec}$. at $\theta= \pm 48.77^{\circ}, 2.5 \mathrm{sec}$. at $\theta=-84.25^{\circ}$ and $\theta=105.75^{\circ}$.

Rotation frames were integrated using CrysAlisPro ${ }^{[11]}$, producing a listing of unaveraged $\mathrm{F}^{2}$ and $\sigma\left(F^{2}\right)$ values. A total of 17806 reflections were measured over the ranges $5.424 \leq 2 \theta \leq 136.39^{\circ},-10 \leq h$ $\leq 10,-6 \leq \mathrm{k} \leq 6,-19 \leq \mathrm{I} \leq 19$ yielding 2843 unique reflections $\left(\mathrm{R}_{\text {int }}=0.0597\right)$. The intensity data were corrected for Lorentz and polarization effects and for absorption using SCALE3 ABSPACK ${ }^{[12]}$ (minimum and maximum transmission $0.6043,1.0000)$. The structure was solved by direct methods - SHELXT ${ }^{[13]}$. Refinement was by full-matrix least squares based on $\mathrm{F}^{2}$ using SHELXL-2018 ${ }^{[14]}$. All reflections were used during refinement. The weighting scheme used was $w=1 /\left[\sigma^{2}\left(F_{o}{ }^{2}\right)+(0.0461 P)^{2}+0.0367 P\right]$ where $P$ $=\left(\mathrm{F}_{\mathrm{o}}^{2}+2 \mathrm{~F}_{\mathrm{c}}^{2}\right) / 3$. Non-hydrogen atoms were refined anisotropically and hydrogen atoms were refined 
using a riding model. Refinement converged to R1=0.0295 and wR2=0.0744 for 2708 observed reflections for which $F>4 \sigma(F)$ and $R 1=0.0310$ and $w R 2=0.0753$ and $G O F=1.066$ for all 2843 unique, non-zero reflections and 284 variables. The maximum $\Delta / \sigma$ in the final cycle of least squares was 0.000 and the two most prominent peaks in the final difference Fourier were +0.12 and $-0.14 \mathrm{e} / \AA^{3}$.

Table 1. lists cell information, data collection parameters, and refinement data. Final positional and equivalent isotropic thermal parameters are given in Tables 2. and 3. Anisotropic thermal parameters are in Table 4. Tables 5. and 6. list bond distances and bond angles. Figure 1. is an ORTEP representation of the molecule with $50 \%$ probability thermal ellipsoids displayed.

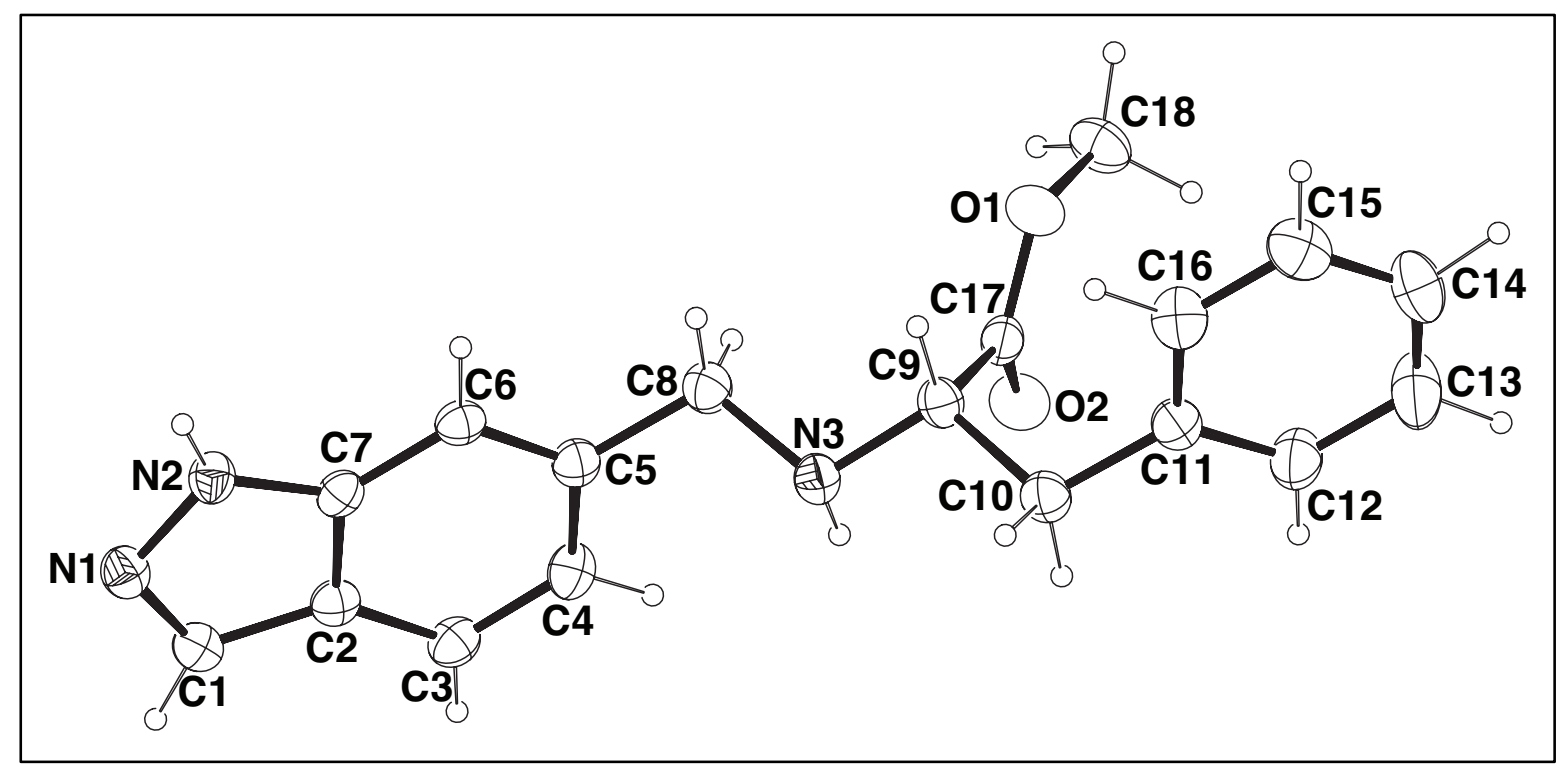

Figure S4. ORTEP drawing of the title compound with $50 \%$ thermal ellipsoids. 
Table 1. Summary of Structure Determination of Compound 2i

CCDC

Empirical formula

Formula weight

Diffractometer

Temperature/K

Crystal system

Space group

a

b

C

$\beta$

Volume

Z

$\mathrm{d}_{\text {calc }}$

$\mu$

$\mathrm{F}(000)$

Crystal size, $\mathrm{mm}$

$2 \theta$ range for data collection

Index ranges

Reflections collected

Independent reflections

Data/restraints/parameters

Goodness-of-fit on $\mathrm{F}^{2}$

Final $R$ indexes $[I>=2 \sigma(I)]$

Final $R$ indexes [all data]

Largest diff. peak/hole

Flack parameter
2075097

$\mathrm{C}_{18} \mathrm{H}_{19} \mathrm{~N}_{3} \mathrm{O}_{2}$

309.36

Rigaku XtaLAB Synergy-S

100.00

monoclinic

$\mathrm{P} 2{ }_{1}$

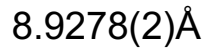

$5.45340(10) \AA$

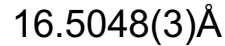

$99.231(2)^{\circ}$

793.16(3) $\AA^{3}$

2

$1.295 \mathrm{~g} / \mathrm{cm}^{3}$

$0.695 \mathrm{~mm}^{-1}$

328.0

$0.45 \times 0.04 \times 0.02$

$5.424-136.39^{\circ}$

$-10 \leq \mathrm{h} \leq 10,-6 \leq \mathrm{k} \leq 6,-19 \leq \mathrm{I} \leq 19$

17806

$2843[\mathrm{R}$ (int) $=0.0597]$

$2843 / 1 / 284$

1.066

$\mathrm{R}_{1}=0.0295, \mathrm{wR}_{2}=0.0744$

$\mathrm{R}_{1}=0.0310, \mathrm{wR}_{2}=0.0753$

$0.12 /-0.14 \mathrm{e}^{-3}$

$0.18(14)$ 
Table 2 . Refined Positional Parameters for Compound 2i

\begin{tabular}{|llllc|}
\hline Atom & \multicolumn{1}{c}{$\boldsymbol{x}$} & \multicolumn{1}{c}{$\boldsymbol{y}$} & \multicolumn{1}{c}{$\boldsymbol{z}$} & $\mathrm{U}(\mathrm{eq})$ \\
\hline \hline $\mathrm{O} 1$ & $0.32805(15)$ & $0.7885(3)$ & $0.85655(7)$ & $0.0292(3)$ \\
O2 & $0.44059(15)$ & $0.4209(3)$ & $0.87615(8)$ & $0.0319(3)$ \\
$\mathrm{N} 1$ & $0.85387(16)$ & $0.3951(3)$ & $0.40527(9)$ & $0.0260(4)$ \\
N2 & $0.75455(16)$ & $0.5542(3)$ & $0.43243(9)$ & $0.0244(3)$ \\
N3 & $0.38378(17)$ & $0.3913(3)$ & $0.69783(9)$ & $0.0247(3)$ \\
C1 & $0.8807(2)$ & $0.2214(4)$ & $0.46145(11)$ & $0.0245(4)$ \\
C2 & $0.79820(19)$ & $0.2632(4)$ & $0.52724(11)$ & $0.0229(4)$ \\
C3 & $0.7838(2)$ & $0.1443(4)$ & $0.60153(12)$ & $0.0258(4)$ \\
C4 & $0.6878(2)$ & $0.2465(4)$ & $0.64957(12)$ & $0.0278(4)$ \\
C5 & $0.6038(2)$ & $0.4623(4)$ & $0.62625(11)$ & $0.0237(4)$ \\
C6 & $0.61762(18)$ & $0.5816(4)$ & $0.55397(11)$ & $0.0228(4)$ \\
C7 & $0.71687(19)$ & $0.4793(3)$ & $0.50541(11)$ & $0.0218(4)$ \\
C8 & $0.5010(2)$ & $0.5682(4)$ & $0.68165(12)$ & $0.0272(4)$ \\
C9 & $0.2793(2)$ & $0.5031(4)$ & $0.74717(11)$ & $0.0234(4)$ \\
C10 & $0.1456(2)$ & $0.3279(4)$ & $0.75222(12)$ & $0.0272(4)$ \\
C11 & $0.0357(2)$ & $0.4315(4)$ & $0.80394(11)$ & $0.0262(4)$ \\
C12 & $0.0169(2)$ & $0.3213(4)$ & $0.87732(13)$ & $0.0349(5)$ \\
C13 & $-0.0831(3)$ & $0.4190(6)$ & $0.92522(15)$ & $0.0457(6)$ \\
C14 & $-0.1642(2)$ & $0.6274(5)$ & $0.90049(14)$ & $0.0419(6)$ \\
C15 & $-0.1455(2)$ & $0.7417(5)$ & $0.82826(14)$ & $0.0375(5)$ \\
C16 & $-0.0463(2)$ & $0.6432(4)$ & $0.78007(13)$ & $0.0336(5)$ \\
C17 & $0.35956(19)$ & $0.5626(4)$ & $0.83374(11)$ & $0.0236(4)$ \\
C18 & $0.3937(2)$ & $0.8557(4)$ & $0.93945(12)$ & $0.0333(5)$ \\
\hline
\end{tabular}


Table 3 . Positional Parameters for Hydrogens in Compound 2i

\begin{tabular}{|lllll|}
\hline Atom & \multicolumn{1}{c}{$\boldsymbol{x}$} & $\boldsymbol{y}$ & \multicolumn{1}{c}{$\boldsymbol{z}$} & $\mathbf{U}(\mathbf{e q})$ \\
\hline $\mathrm{H} 2$ & $0.714(2)$ & $0.675(5)$ & $0.3986(13)$ & $0.027(6)$ \\
$\mathrm{H} 3 \mathrm{a}$ & $0.428(2)$ & $0.269(5)$ & $0.7278(13)$ & $0.027(5)$ \\
$\mathrm{H} 1$ & $0.950(2)$ & $0.091(5)$ & $0.4550(14)$ & $0.030(5)$ \\
$\mathrm{H} 3$ & $0.837(2)$ & $0.003(5)$ & $0.6203(12)$ & $0.025(5)$ \\
$\mathrm{H} 4$ & $0.681(3)$ & $0.170(5)$ & $0.7019(15)$ & $0.038(6)$ \\
$\mathrm{H} 6$ & $0.561(2)$ & $0.733(4)$ & $0.5365(12)$ & $0.022(5)$ \\
$\mathrm{H} 8 \mathrm{a}$ & $0.566(3)$ & $0.623(5)$ & $0.7335(15)$ & $0.036(6)$ \\
$\mathrm{H} 8 \mathrm{~b}$ & $0.446(2)$ & $0.714(4)$ & $0.6559(12)$ & $0.022(5)$ \\
$\mathrm{H} 9$ & $0.239(2)$ & $0.661(4)$ & $0.7204(12)$ & $0.017(5)$ \\
$\mathrm{H} 10 \mathrm{a}$ & $0.091(2)$ & $0.294(5)$ & $0.6947(15)$ & $0.036(6)$ \\
$\mathrm{H} 10 \mathrm{~b}$ & $0.186(2)$ & $0.168(5)$ & $0.7734(13)$ & $0.027(5)$ \\
$\mathrm{H} 12$ & $0.072(3)$ & $0.171(6)$ & $0.8959(16)$ & $0.047(7)$ \\
$\mathrm{H} 13$ & $-0.093(3)$ & $0.327(7)$ & $0.978(2)$ & $0.068(9)$ \\
$\mathrm{H} 14$ & $-0.224(3)$ & $0.705(6)$ & $0.9367(17)$ & $0.060(8)$ \\
$\mathrm{H} 15$ & $-0.205(3)$ & $0.898(6)$ & $0.8103(15)$ & $0.046(7)$ \\
$\mathrm{H} 16$ & $-0.033(3)$ & $0.729(5)$ & $0.7286(16)$ & $0.043(7)$ \\
$\mathrm{H} 18 \mathrm{a}$ & $0.506(3)$ & $0.849(5)$ & $0.9445(15)$ & $0.043(7)$ \\
$\mathrm{H} 18 \mathrm{~b}$ & $0.351(3)$ & $0.744(5)$ & $0.9802(15)$ & $0.036(6)$ \\
$\mathrm{H} 18 \mathrm{c}$ & $0.362(3)$ & $1.024(6)$ & $0.9447(15)$ & $0.042(7)$ \\
\hline
\end{tabular}


Table 4 . Refined Thermal Parameters (U's) for Compound 2i

\begin{tabular}{|lllllll|}
\hline Atom & $\mathbf{U}_{11}$ & $\mathbf{U}_{22}$ & \multicolumn{1}{c}{$\mathbf{U}_{33}$} & \multicolumn{1}{c}{$\mathbf{U}_{23}$} & $\mathbf{U}_{13}$ & $\mathbf{U}_{12}$ \\
\hline \hline $\mathrm{O} 1$ & $0.0342(7)$ & $0.0278(8)$ & $0.0238(6)$ & $-0.0040(6)$ & $-0.0006(5)$ & $0.0026(6)$ \\
$\mathrm{O} 2$ & $0.0349(7)$ & $0.0325(8)$ & $0.0263(7)$ & $0.0017(6)$ & $-0.0014(5)$ & $0.0051(6)$ \\
$\mathrm{N} 1$ & $0.0232(7)$ & $0.0296(9)$ & $0.0253(8)$ & $-0.0020(7)$ & $0.0037(6)$ & $-0.0004(7)$ \\
$\mathrm{N} 2$ & $0.0238(7)$ & $0.0268(9)$ & $0.0227(7)$ & $0.0033(7)$ & $0.0042(6)$ & $0.0013(7)$ \\
$\mathrm{N} 3$ & $0.0247(7)$ & $0.0273(9)$ & $0.0226(7)$ & $-0.0006(7)$ & $0.0053(6)$ & $-0.0004(7)$ \\
$\mathrm{C} 1$ & $0.0218(8)$ & $0.0255(11)$ & $0.0258(9)$ & $-0.0023(8)$ & $0.0021(7)$ & $0.0009(8)$ \\
$\mathrm{C} 2$ & $0.0195(8)$ & $0.0227(9)$ & $0.0257(9)$ & $-0.0005(8)$ & $0.0012(7)$ & $-0.0016(7)$ \\
$\mathrm{C} 3$ & $0.0239(8)$ & $0.0242(10)$ & $0.0288(9)$ & $0.0045(8)$ & $0.0021(7)$ & $0.0008(8)$ \\
$\mathrm{C} 4$ & $0.0271(9)$ & $0.0315(11)$ & $0.0253(9)$ & $0.0051(8)$ & $0.0052(7)$ & $-0.0005(8)$ \\
$\mathrm{C} 5$ & $0.0217(8)$ & $0.0253(10)$ & $0.0239(9)$ & $-0.0020(7)$ & $0.0028(7)$ & $-0.0036(7)$ \\
$\mathrm{C} 6$ & $0.0204(8)$ & $0.0206(9)$ & $0.0266(9)$ & $0.0000(8)$ & $0.0014(7)$ & $-0.0013(8)$ \\
$\mathrm{C} 7$ & $0.0201(8)$ & $0.0228(9)$ & $0.0218(8)$ & $0.0006(7)$ & $0.0009(6)$ & $-0.0037(7)$ \\
$\mathrm{C} 8$ & $0.0260(9)$ & $0.0282(10)$ & $0.0281(9)$ & $-0.0022(8)$ & $0.0064(7)$ & $-0.0022(8)$ \\
$\mathrm{C} 9$ & $0.0229(9)$ & $0.0271(10)$ & $0.0199(8)$ & $-0.0008(7)$ & $0.0029(7)$ & $0.0002(7)$ \\
$\mathrm{C} 10$ & $0.0261(9)$ & $0.0293(11)$ & $0.0262(9)$ & $-0.0044(8)$ & $0.0043(7)$ & $-0.0032(8)$ \\
$\mathrm{C} 11$ & $0.0208(8)$ & $0.0296(11)$ & $0.0278(9)$ & $-0.0048(8)$ & $0.0029(7)$ & $-0.0040(8)$ \\
$\mathrm{C} 12$ & $0.0326(10)$ & $0.0361(12)$ & $0.0381(11)$ & $0.0062(9)$ & $0.0121(9)$ & $0.0034(9)$ \\
$\mathrm{C} 13$ & $0.0415(12)$ & $0.0591(16)$ & $0.0411(12)$ & $0.0087(12)$ & $0.0203(10)$ & $0.0064(12)$ \\
$\mathrm{C} 14$ & $0.0318(10)$ & $0.0555(16)$ & $0.0410(12)$ & $-0.0036(11)$ & $0.0140(9)$ & $0.007(1)$ \\
$\mathrm{C} 15$ & $0.0272(10)$ & $0.0404(13)$ & $0.0434(11)$ & $-0.0032(11)$ & $0.0012(8)$ & $0.0057(9)$ \\
$\mathrm{C} 16$ & $0.0303(10)$ & $0.0395(13)$ & $0.0303(10)$ & $0.0031(9)$ & $0.0030(8)$ & $0.0026(9)$ \\
$\mathrm{C} 17$ & $0.0240(8)$ & $0.0256(10)$ & $0.0220(8)$ & $0.0007(8)$ & $0.0067(7)$ & $-0.0030(8)$ \\
$\mathrm{C} 18$ & $0.0397(11)$ & $0.0343(13)$ & $0.0243(9)$ & $-0.0073(9)$ & $0.0003(8)$ & $0.0029(9)$ \\
\hline
\end{tabular}


Table 5 . Bond Distances in Compound 2i, $\AA$

\begin{tabular}{|llllll|}
\hline $\mathrm{O} 1-\mathrm{C} 17$ & $1.331(2)$ & $\mathrm{O} 1-\mathrm{C} 18$ & $1.447(2)$ & $\mathrm{O} 2-\mathrm{C} 17$ & $1.203(2)$ \\
$\mathrm{N} 1-\mathrm{N} 2$ & $1.367(2)$ & $\mathrm{N} 1-\mathrm{C} 1$ & $1.320(3)$ & $\mathrm{N} 2-\mathrm{H} 2$ & $0.90(2)$ \\
$\mathrm{N} 2-\mathrm{C} 7$ & $1.364(2)$ & $\mathrm{N} 3-\mathrm{H} 3 \mathrm{a}$ & $0.88(3)$ & $\mathrm{N} 3-\mathrm{C} 8$ & $1.479(3)$ \\
$\mathrm{N} 3-\mathrm{C} 9$ & $1.466(2)$ & $\mathrm{C} 1-\mathrm{H} 1$ & $0.96(2)$ & $\mathrm{C} 1-\mathrm{C} 2$ & $1.425(3)$ \\
$\mathrm{C} 2-\mathrm{C} 3$ & $1.411(3)$ & $\mathrm{C} 2-\mathrm{C} 7$ & $1.401(3)$ & $\mathrm{C} 3-\mathrm{H} 3$ & $0.93(2)$ \\
$\mathrm{C} 3-\mathrm{C} 4$ & $1.375(3)$ & $\mathrm{C} 4-\mathrm{H} 4$ & $0.97(3)$ & $\mathrm{C} 4-\mathrm{C} 5$ & $1.415(3)$ \\
$\mathrm{C} 5-\mathrm{C} 6$ & $1.382(3)$ & $\mathrm{C} 5-\mathrm{C} 8$ & $1.510(2)$ & $\mathrm{C} 6-\mathrm{H} 6$ & $0.98(2)$ \\
$\mathrm{C} 6-\mathrm{C} 7$ & $1.402(3)$ & $\mathrm{C} 8-\mathrm{H} 8 \mathrm{a}$ & $1.00(2)$ & $\mathrm{C} 8-\mathrm{H} 8 \mathrm{~b}$ & $0.99(2)$ \\
$\mathrm{C} 9-\mathrm{H} 9$ & $1.01(2)$ & $\mathrm{C}-\mathrm{C} 10$ & $1.542(3)$ & $\mathrm{C} 9-\mathrm{C} 17$ & $1.527(2)$ \\
$\mathrm{C} 10-\mathrm{H} 10 \mathrm{a} 1.01(2)$ & $\mathrm{C} 10-\mathrm{H} 10 \mathrm{~b}$ & $0.98(2)$ & $\mathrm{C} 10-\mathrm{C} 11$ & $1.510(3)$ \\
$\mathrm{C} 11-\mathrm{C} 12$ & $1.386(3)$ & $\mathrm{C} 11-\mathrm{C} 16$ & $1.390(3)$ & $\mathrm{C} 12-\mathrm{H} 12$ & $0.98(3)$ \\
$\mathrm{C} 12-\mathrm{C} 13$ & $1.390(3)$ & $\mathrm{C} 13-\mathrm{H} 13$ & $1.02(3)$ & $\mathrm{C} 13-\mathrm{C} 14$ & $1.374(4)$ \\
$\mathrm{C} 14-\mathrm{H} 14$ & $0.96(3)$ & $\mathrm{C} 14-\mathrm{C} 15$ & $1.379(3)$ & $\mathrm{C} 15-\mathrm{H} 15$ & $1.02(3)$ \\
$\mathrm{C} 15-\mathrm{C} 16$ & $1.390(3)$ & $\mathrm{C} 16-\mathrm{H} 16$ & $0.99(3)$ & $\mathrm{C} 18-\mathrm{H} 18 a$ & $0.99(2)$ \\
$\mathrm{C} 18-\mathrm{H} 18 \mathrm{~b} 1.02(3)$ & $\mathrm{C} 18-\mathrm{H} 18 \mathrm{c}$ & $0.97(3)$ & & \\
\hline
\end{tabular}


Table 6 . Bond Angles in Compound 2i, ${ }^{\circ}$

\begin{tabular}{|c|c|c|c|c|c|}
\hline C17-01-C18 & $115.45(15)$ & C1-N1-N2 & $106.21(15)$ & $\mathrm{N} 1-\mathrm{N} 2-\mathrm{H} 2$ & $118.5(14)$ \\
\hline C7-N2-N1 & $111.38(16)$ & $\mathrm{C} 7-\mathrm{N} 2-\mathrm{H} 2$ & $129.4(14)$ & C8-N3-H3a & $109.3(14)$ \\
\hline C9-N3-H3a & $105.5(14)$ & C9-N3-C8 & $11.14(15)$ & $\mathrm{N} 1-\mathrm{C} 1-\mathrm{H} 1$ & $120.0(14)$ \\
\hline $\mathrm{N} 1-\mathrm{C} 1-\mathrm{C} 2$ & $111.29(17)$ & $\mathrm{C} 2-\mathrm{C} 1-\mathrm{H} 1$ & $128.7(14)$ & C3-C2-C1 & (18) \\
\hline $\mathrm{C} 7-\mathrm{C} 2-\mathrm{C} 1$ & $104.38(16)$ & $\mathrm{C} 7-\mathrm{C} 2-\mathrm{C} 3$ & $119.62(17)$ & $\mathrm{C} 2-\mathrm{C} 3-\mathrm{H} 3$ & $123.8(13)$ \\
\hline C4-C3-C2 & $117.85(18)$ & $\mathrm{C} 4-\mathrm{C} 3-\mathrm{H} 3$ & $118.3(13)$ & $\mathrm{C} 3-\mathrm{C} 4-\mathrm{H} 4$ & (15) \\
\hline C3- & 122.1 & $\mathrm{C} 5-\mathrm{C}$ & 119. & $-\mathrm{C} 8$ & (16) \\
\hline C6-C & 120.6 & C6- & |8) & $\mathrm{H} 6$ & \\
\hline C5-C & 117.3 & $\mathrm{C} 7-\mathrm{C}$ & 120.4 & 2 & $3(16)$ \\
\hline N2-C7-C6 & $130.82(18)$ & C2-C7-C6 & $122.45(17)$ & N3-C8-C5 & $112.09(16)$ \\
\hline N3-C8-H8a & $112.0(14)$ & N3-C & 106. & $\mathrm{H} 8 \mathrm{a}$ & 14) \\
\hline C5-C & 110 & $-\mathrm{H} 8 \mathrm{~b}$ & 107 & $-H 9$ & 11) \\
\hline N3-C9-C10 & $109.84(15)$ & N3-C9-C17 & 111. & C10-C9-H9 & 10 \\
\hline C17-C9-H9 & $108.1(12)$ & C17-C9-C10 & $109.39(14)$ & C9-C10-H10a & (14) \\
\hline $\mathrm{C} 9-\mathrm{C} 10-\mathrm{H} 10 \mathrm{~b}$ & $109.2(13)$ & $\mathrm{H} 10 \mathrm{a}-\mathrm{C} 10-\mathrm{H} 10 \mathrm{~b}$ & $105(2)$ & C11-C10-C9 & $112.21(16)$ \\
\hline C11-C10-H10a & 109.8 & 0-H10b & 111 & $11-C 10$ & (18) \\
\hline C12-C11-C16 & 118.3 & $1-C 10$ & 120.7 & 12-H12 & 12 \\
\hline C11-C12-C13 & $120.7(2)$ & C13-C12-H12 & $118.6(16)$ & $\mathrm{C} 12-\mathrm{C} 13-\mathrm{H} 13$ & $116.7(19)$ \\
\hline C14-C13-C12 & $120.3(2)$ & C14-C13-H13 & 123.0 & C13-C14-H14 & $120.0(19)$ \\
\hline C13-C14-C15 & $119.9(2)$ & C15-C14-H14 & 119.6(19) & C14-C15-H15 & $119.9(14)$ \\
\hline C14-C15-C16 & $119.8(2)$ & C16-C15-H15 & $120.3(14)$ & C11-C16-C15 & $120.9(2)$ \\
\hline C11-C16-H16 & $120.0(16)$ & C15-C16-H16 & $119.0(16)$ & O1-C17-C9 & $111.87(15)$ \\
\hline O2-C17-O1 & $124.36(17)$ & O2-C17-C9 & $123.77(18)$ & O1-C18-H18a & $108.6(14)$ \\
\hline O1-C18-H18b & $109.5(14)$ & O1-C18-H18c & $104.2(15)$ & $\mathrm{H} 18 \mathrm{a}-\mathrm{C} 18-\mathrm{H} 18 \mathrm{~b}$ & $114(2)$ \\
\hline \multicolumn{2}{|c|}{$\mathrm{H} 18 \mathrm{a}-\mathrm{C} 18-\mathrm{H} 18 \mathrm{c}$ 109(2) } & \multicolumn{4}{|c|}{$\mathrm{H} 18 \mathrm{~b}-\mathrm{C} 18-\mathrm{H} 18 \mathrm{c} 111(2)$} \\
\hline
\end{tabular}

This report has been created with Olex2 ${ }^{[15]}$, compiled on 2019.03 .18 svn.r3573 for OlexSys. 


\section{References}

[1] N. R. Patel, C. B. Kelly, M. Jouffroy, G. A. Molander, Org. Lett. 2016, 18, 764-767.

[2] M. Durandetti, M. Devaud, J. Périchon. New J. Chem. 1996, 20, 659-667.

[3] C. B. Kelly, N. R. Patel, D. N. Primer, M. Jouffroy, J. C. Tellis, G. A. Molander, Nat. Protoc. 2017, 12, 472-492.

[4] N. R. Patel, C. B. Kelly, A. P. Siegenfeld, G. A. Molander, ACS Catal. 2017, 7, 1766-1770.

[5] S. G. Koller, J. O. Bauer, C. Strohmann, Angew. Chem. Int. Ed. 2017, 56, 7991-7994.

[6] J. Li, Y. Sha, Molecules 2008, 13, 1111-1119.

[7] J. B. Roque, Y. Kuroda, L. T. Göttemann, R. Sarpong, Science 2018, 361, 171-174.

[8] F. Cong, X. Lv, C. S. Day, R. Martin J. Am. Chem. Soc. 2020 142, 20594-20599.

[9] C. Remeur, C. B. Kelly, N. R. Patel, G. A. Molander, Aminomethylation of Aryl Halides Using aSilylamines Enabled by Ni/Photoredox Dual Catalysis. ACS Catal. 2017, 7, 6065-6069.

[10] CrysAlisPro 1.171.40.81a: Rigaku Oxford Diffraction, Rigaku Corporation, Oxford, UK. (2019).

[11] CrysAlisPro 1.171.40.81a: Rigaku Oxford Diffraction, Rigaku Corporation, Oxford, UK. (2019).

[12] SCALE3 ABSPACK v1.0.7: an Oxford Diffraction program; Oxford Diffraction Ltd: Abingdon, UK, 2005.

[13] SHELXT v2014/4: G. Sheldrick, Acta Crystallographica Section A 2015, 71, 3-8.

[14] SHELXL-2018/3 G. Sheldrick, Acta Crystallographica Section A 2015, 71, 3-8.

[15] Olex2: O. V. Dolomanov, L. J. Bourhis, R. J. Gildea, J. A. Howard, H. Puschmann, J. Appl. Crystallogr. 2009, 42, 339-341. 


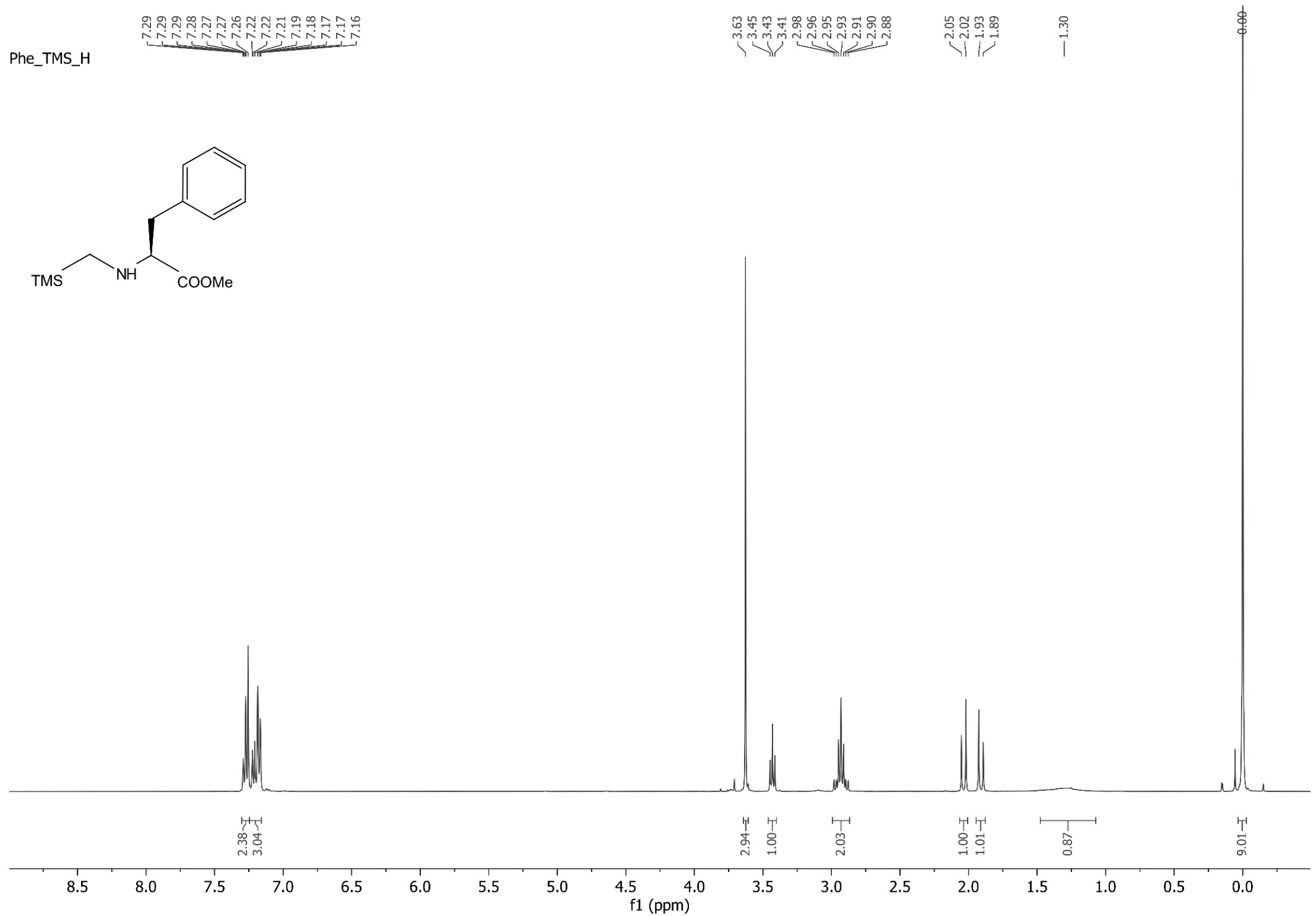


$\left.{ }^{13} \mathrm{C} \mathrm{NMR} \mathrm{(101} \mathrm{MHz,} \mathrm{CDCl}_{3}\right)$ spectrum of 1a

Phe_TMS_C

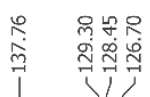

0
0
0

$\stackrel{\mathscr{8}}{4}$
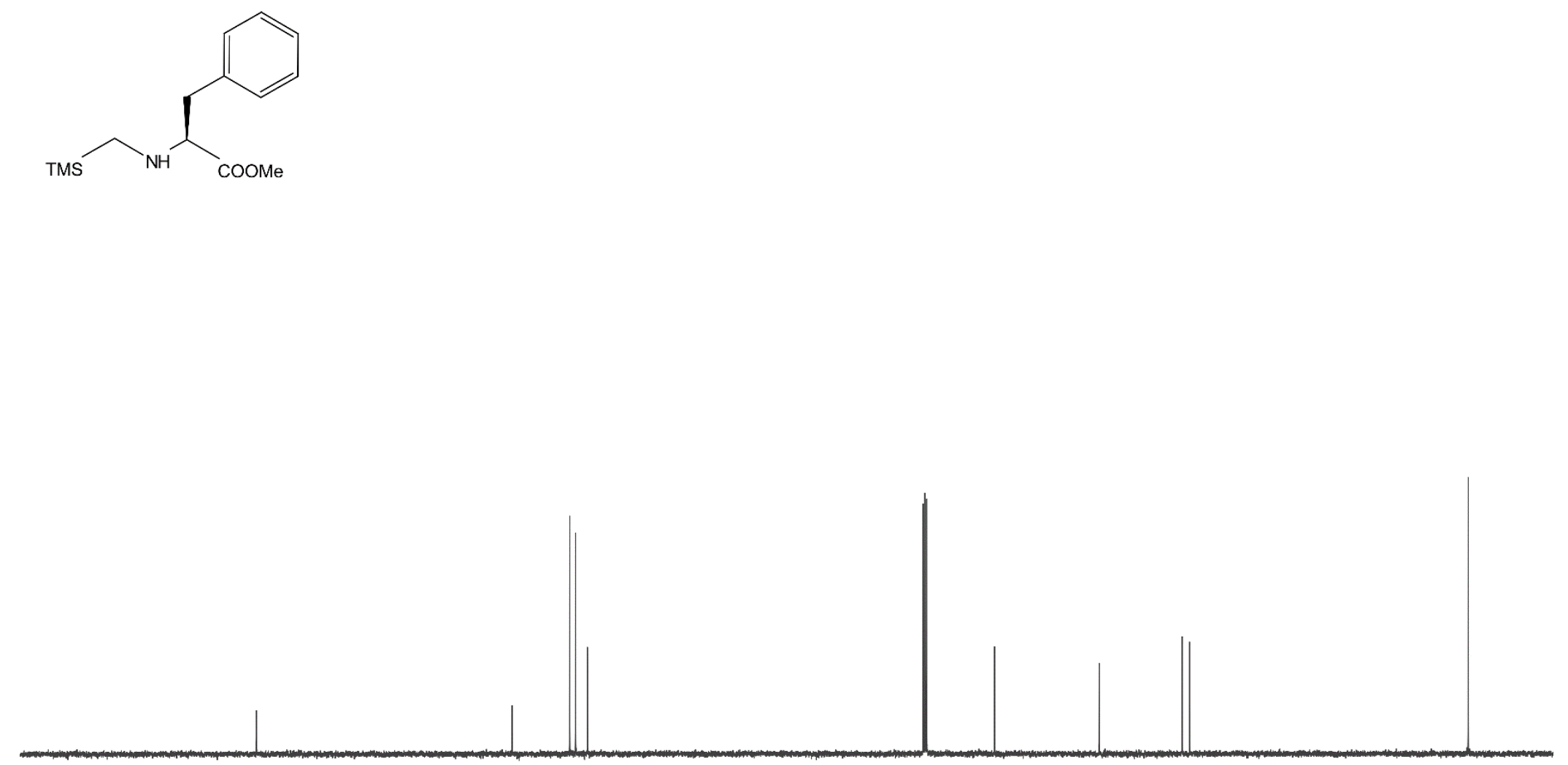

$10 \quad 200$

$200 \quad 190$

$\begin{array}{lll}180 & 170\end{array}$

$160 \quad 150$

$140 \quad 130$

120

$10 \quad 100 \quad 90$

1 (ppm)

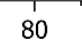

$70 \quad 60 \quad 50$
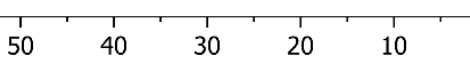

$-10$ 
${ }^{1} \mathrm{H}$ NMR $\left(500 \mathrm{MHz}, \mathrm{CDCl}_{3}\right)$ spectrum of $\mathbf{1 b}$

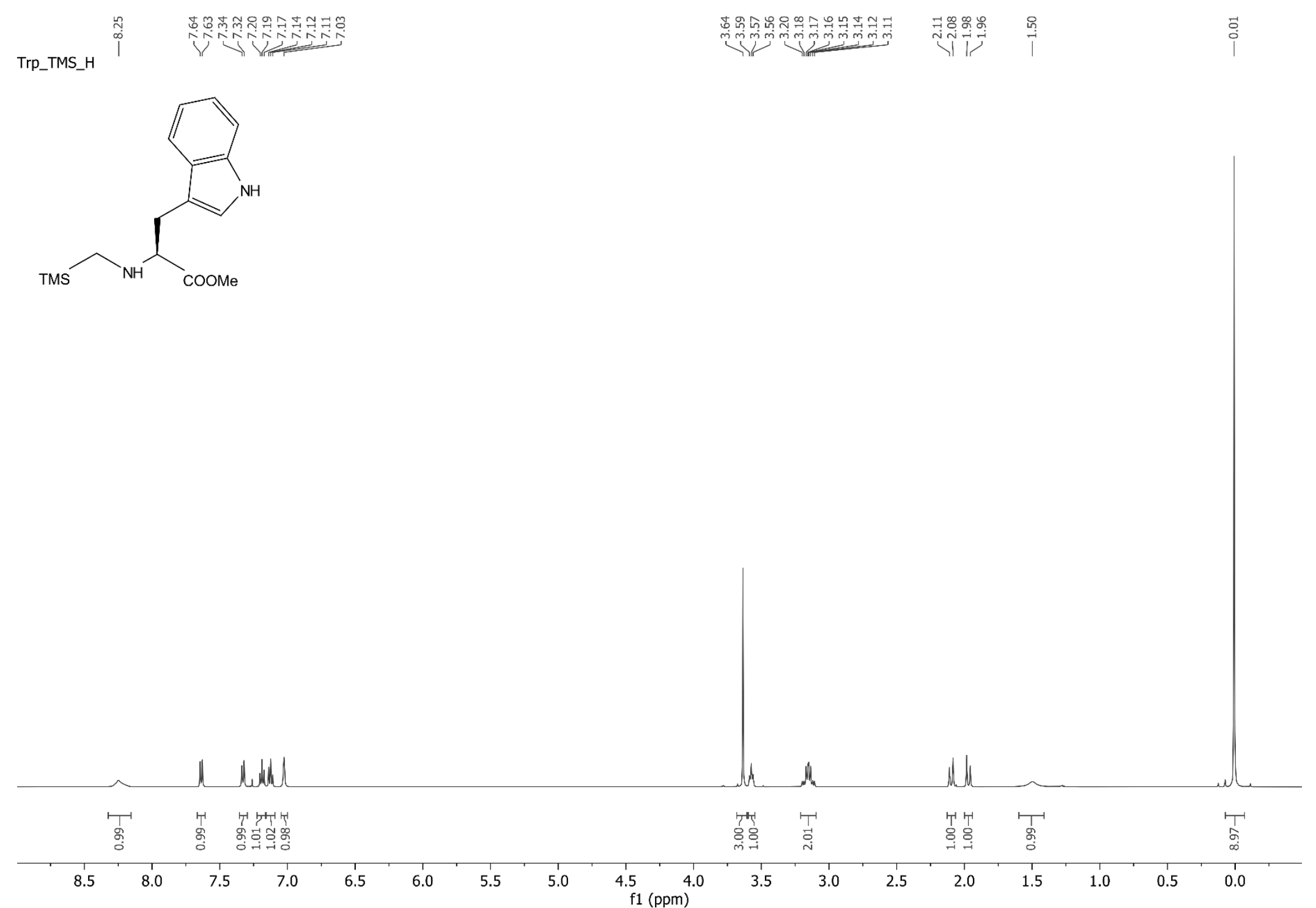




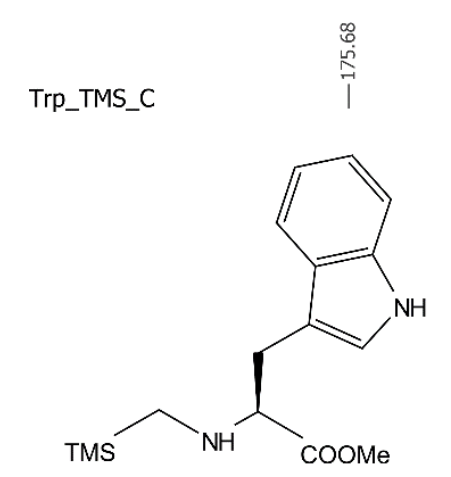

${ }^{13} \mathrm{C}$ NMR $\left(126 \mathrm{MHz}, \mathrm{CDCl}_{3}\right)$ spectrum of $\mathbf{1 b}$

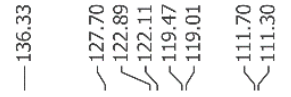
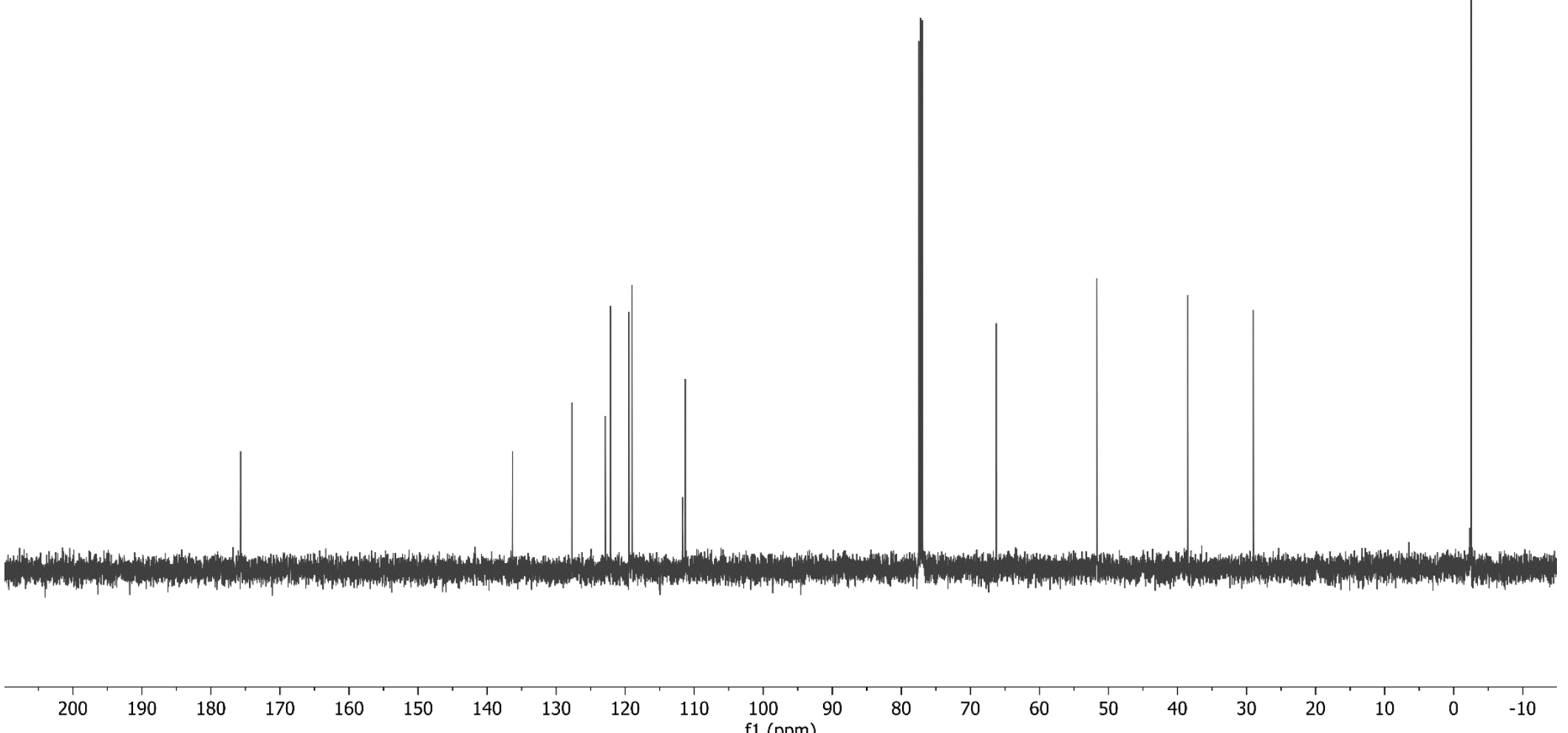
${ }^{1} \mathrm{H}$ NMR $\left(500 \mathrm{MHz}, \mathrm{CDCl}_{3}\right)$ spectrum of 1c

Met_TMS_H
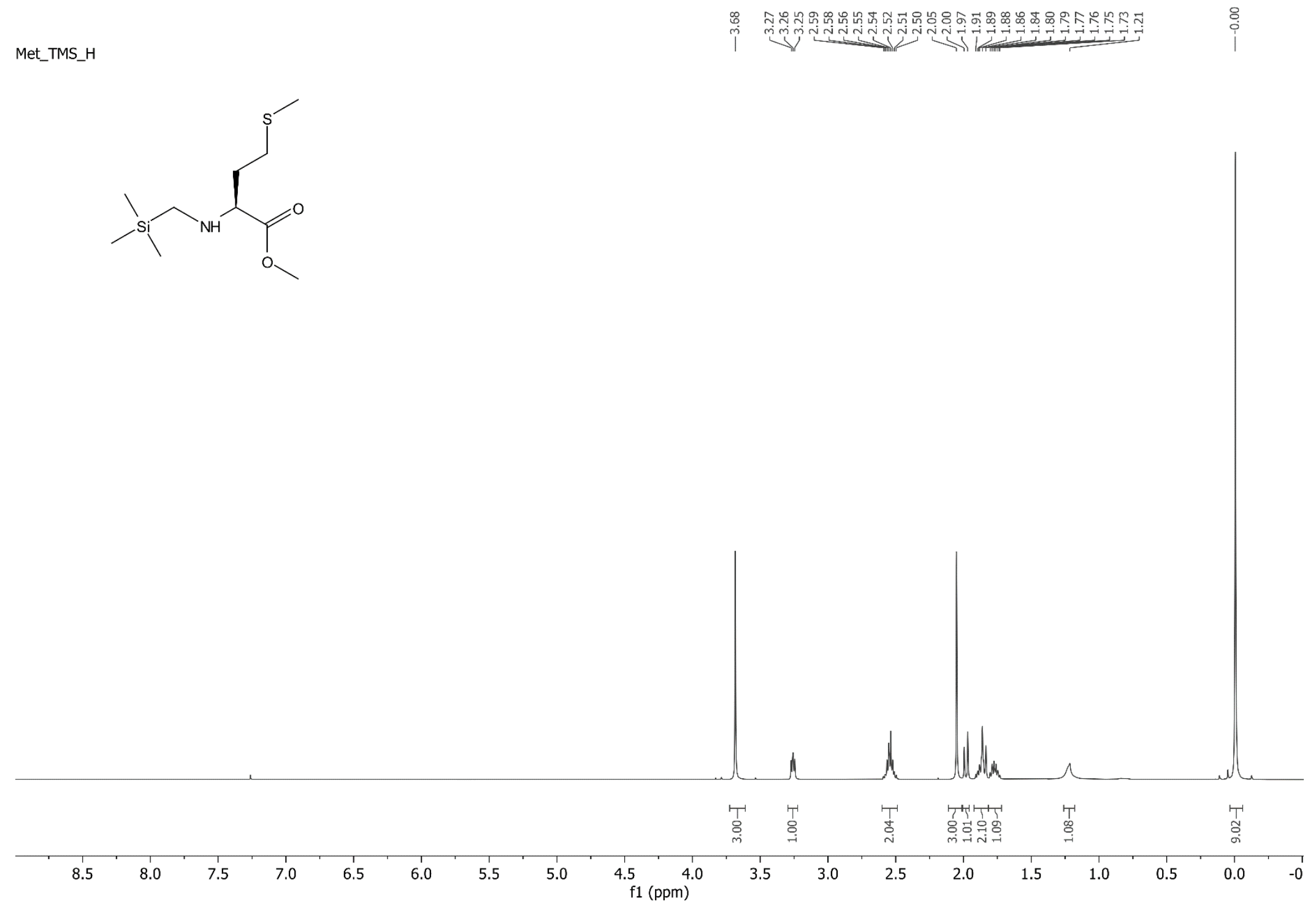
$\left.{ }^{13} \mathrm{C} \mathrm{NMR} \mathrm{(126} \mathrm{MHz,} \mathrm{CDCl}_{3}\right)$ spectrum of 1c

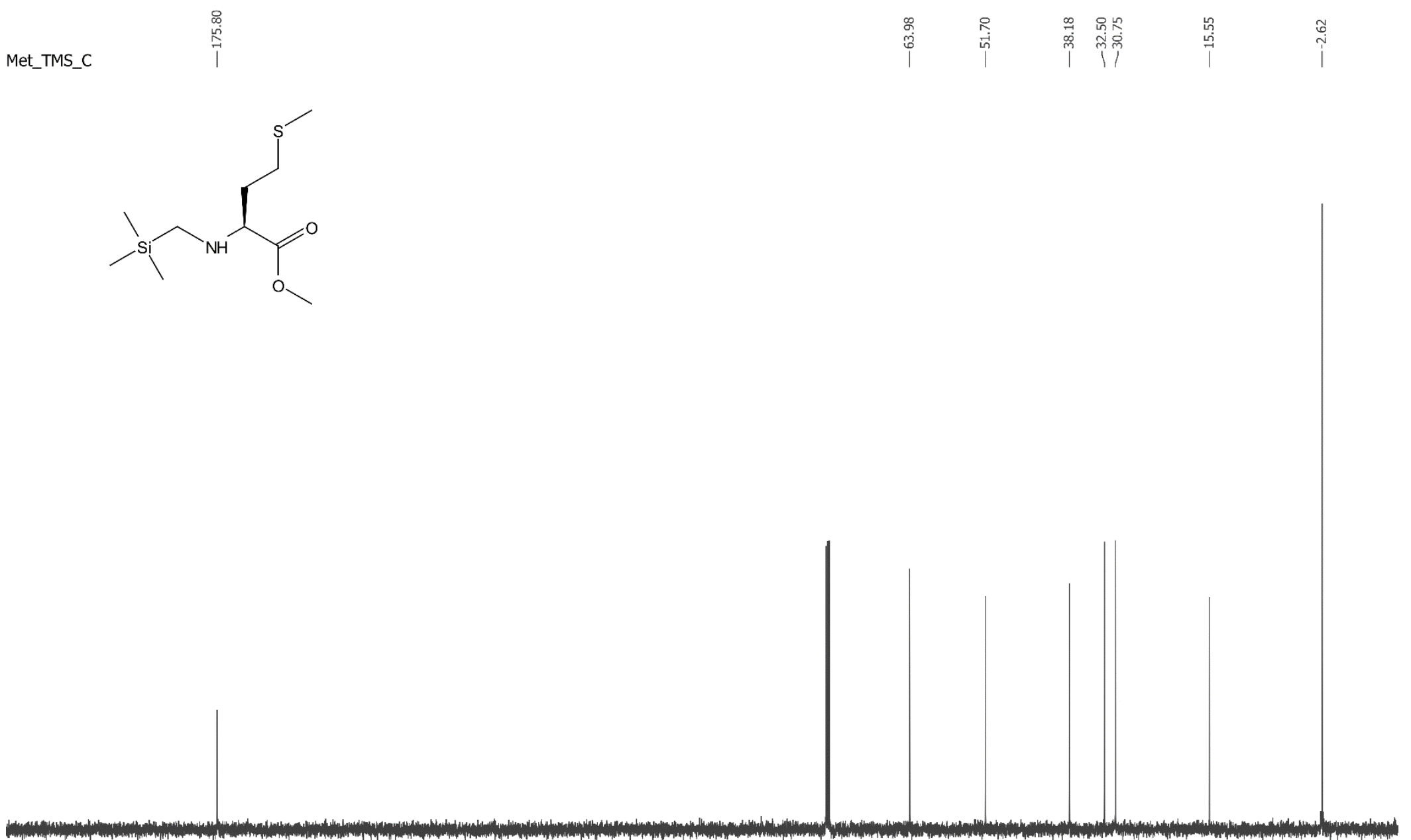

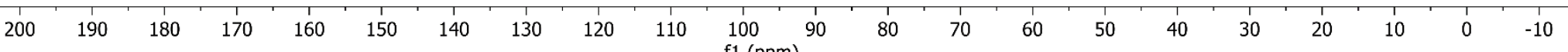


${ }^{1} \mathrm{H}$ NMR $\left(500 \mathrm{MHz}, \mathrm{CDCl}_{3}\right.$ ) spectrum of $\mathbf{1 d}$

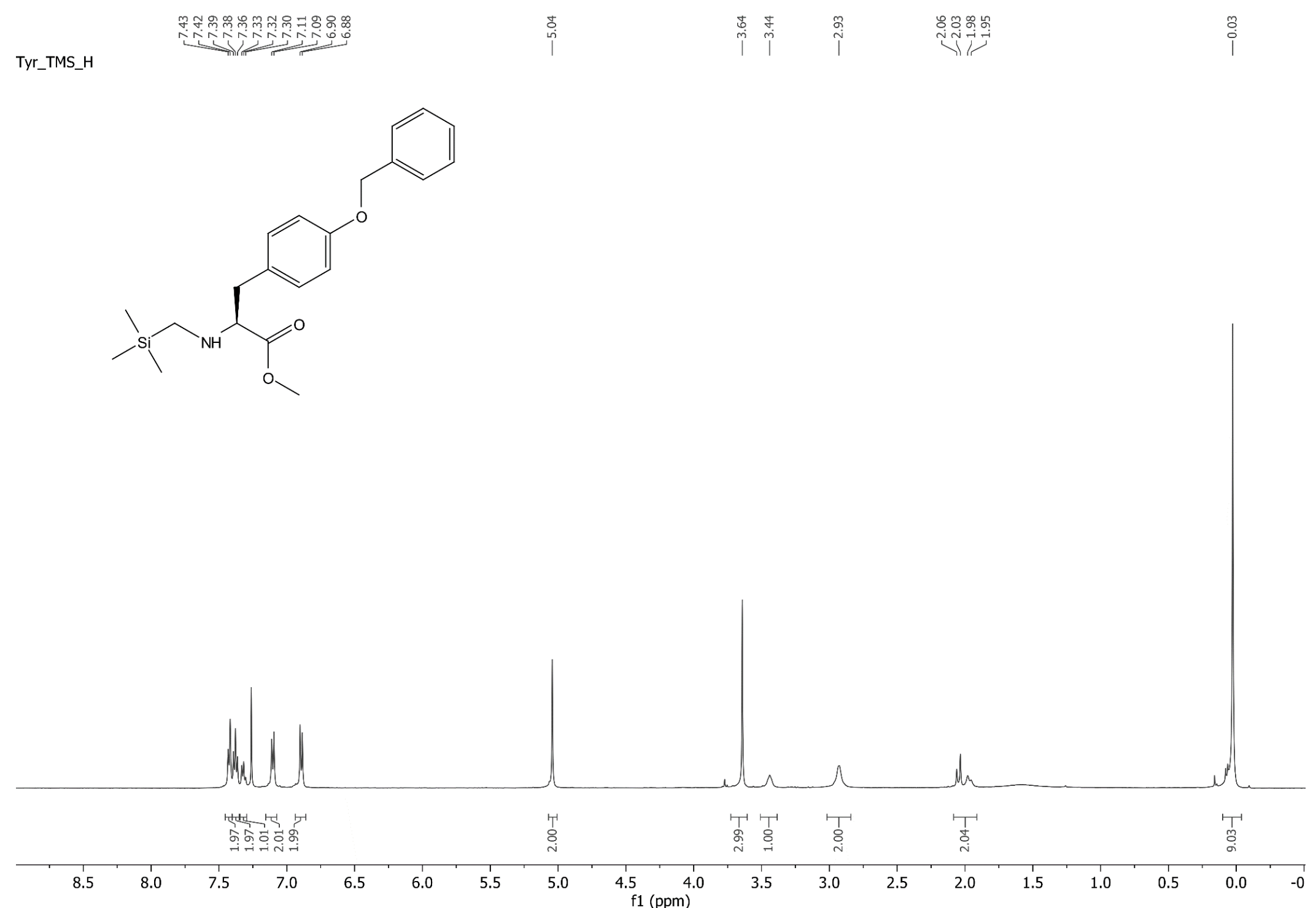


${ }^{13} \mathrm{C}$ NMR $\left(126 \mathrm{MHz}, \mathrm{CDCl}_{3}\right)$ spectrum of 1d

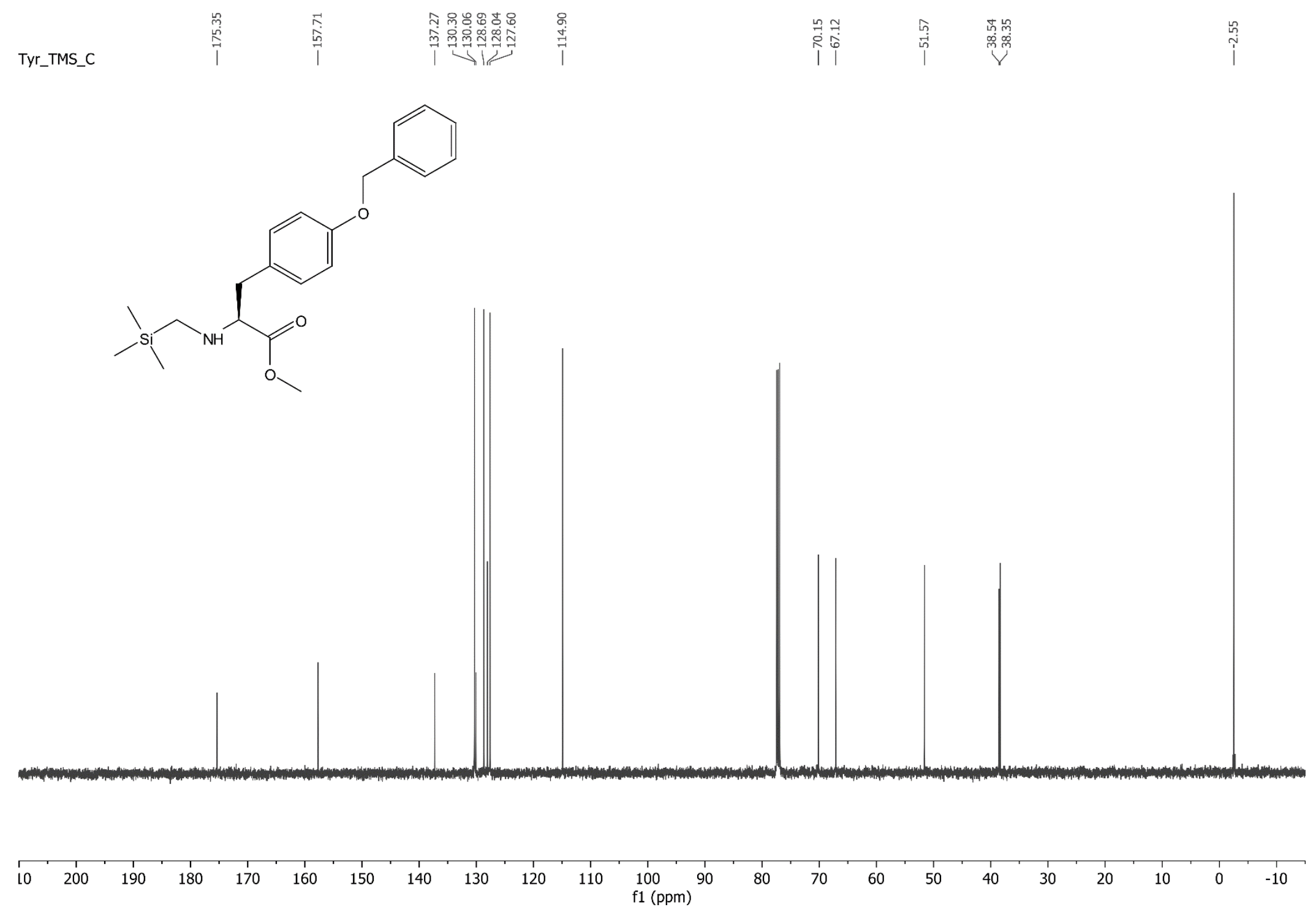


${ }^{1} \mathrm{H}$ NMR $\left(500 \mathrm{MHz}, \mathrm{CDCl}_{3}\right)$ spectrum of $\mathbf{1 e}$

Leu_TMS_H
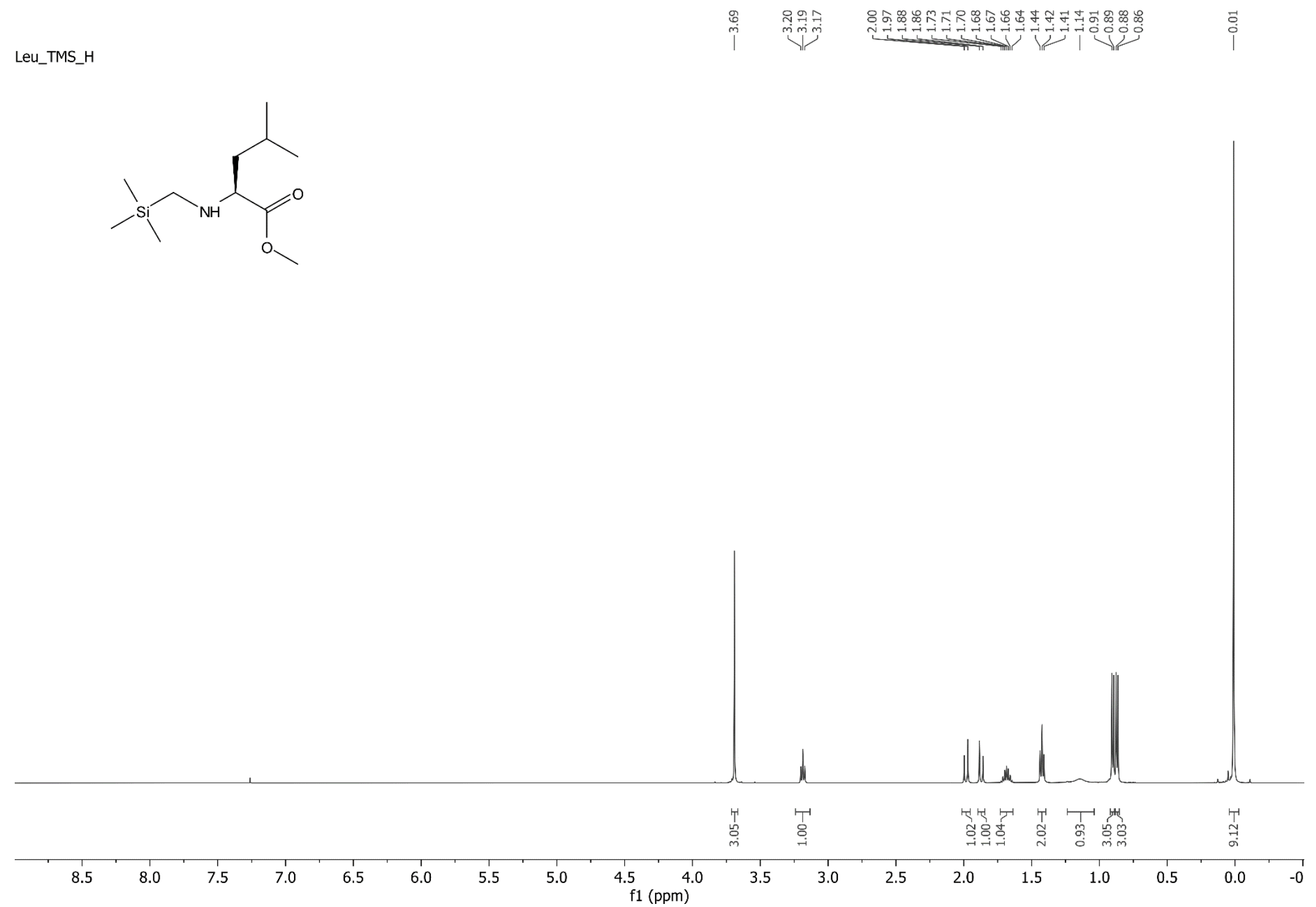
${ }^{13} \mathrm{H}$ NMR $\left(126 \mathrm{MHz}, \mathrm{CDCl}_{3}\right.$ ) spectrum of $\mathbf{1 e}$

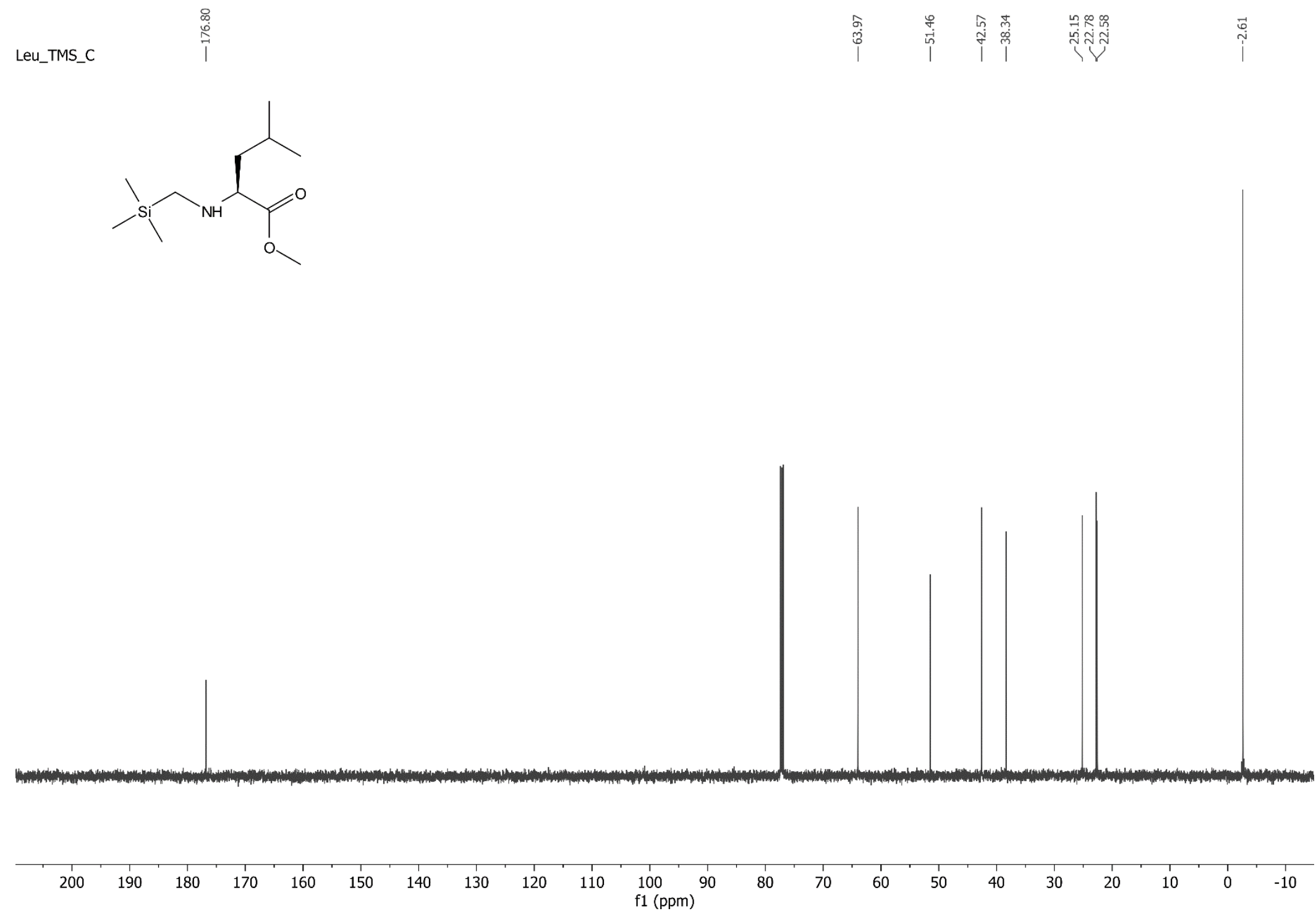


${ }^{1} \mathrm{H}$ NMR $\left(500 \mathrm{MHz}, \mathrm{CDCl}_{3}\right)$ spectrum of $1 \mathrm{f}$

Gly_TMS_H

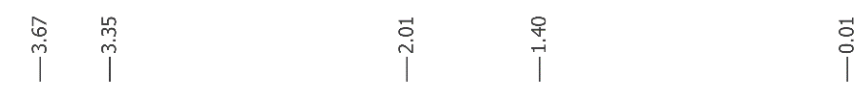<smiles>COC(=O)CNC[Si](C)(C)C</smiles>

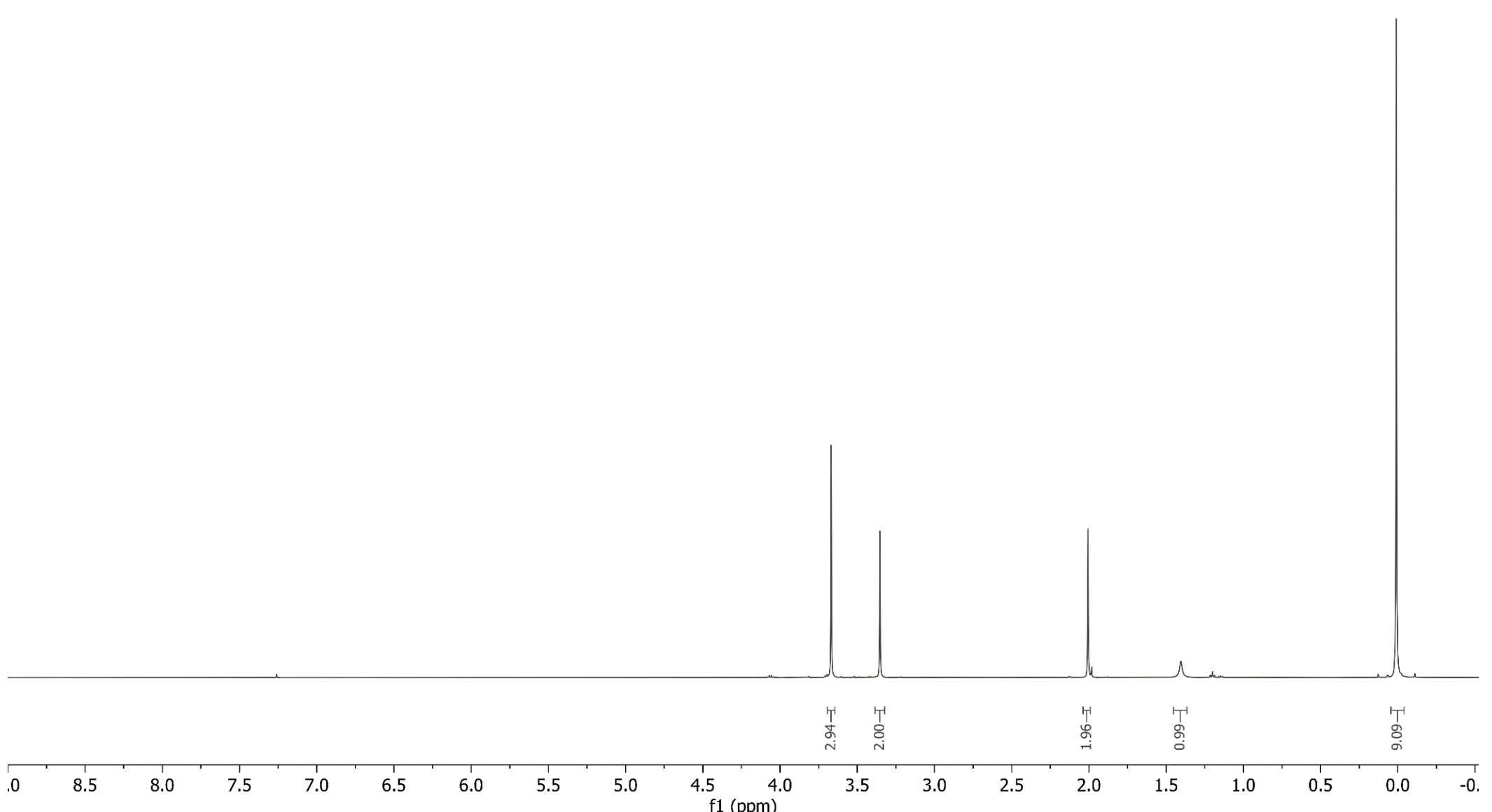


${ }^{13} \mathrm{C} \mathrm{NMR} \mathrm{(126} \mathrm{MHz,} \mathrm{CDCl}_{3}$ ) spectrum of 1 f

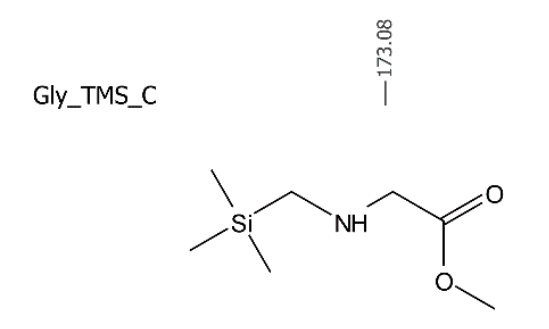

NMR (126 MHz, $\left.\mathrm{CDCl}_{3}\right)$ spectrum of $1 \mathrm{f}$

Gly_TMS_C

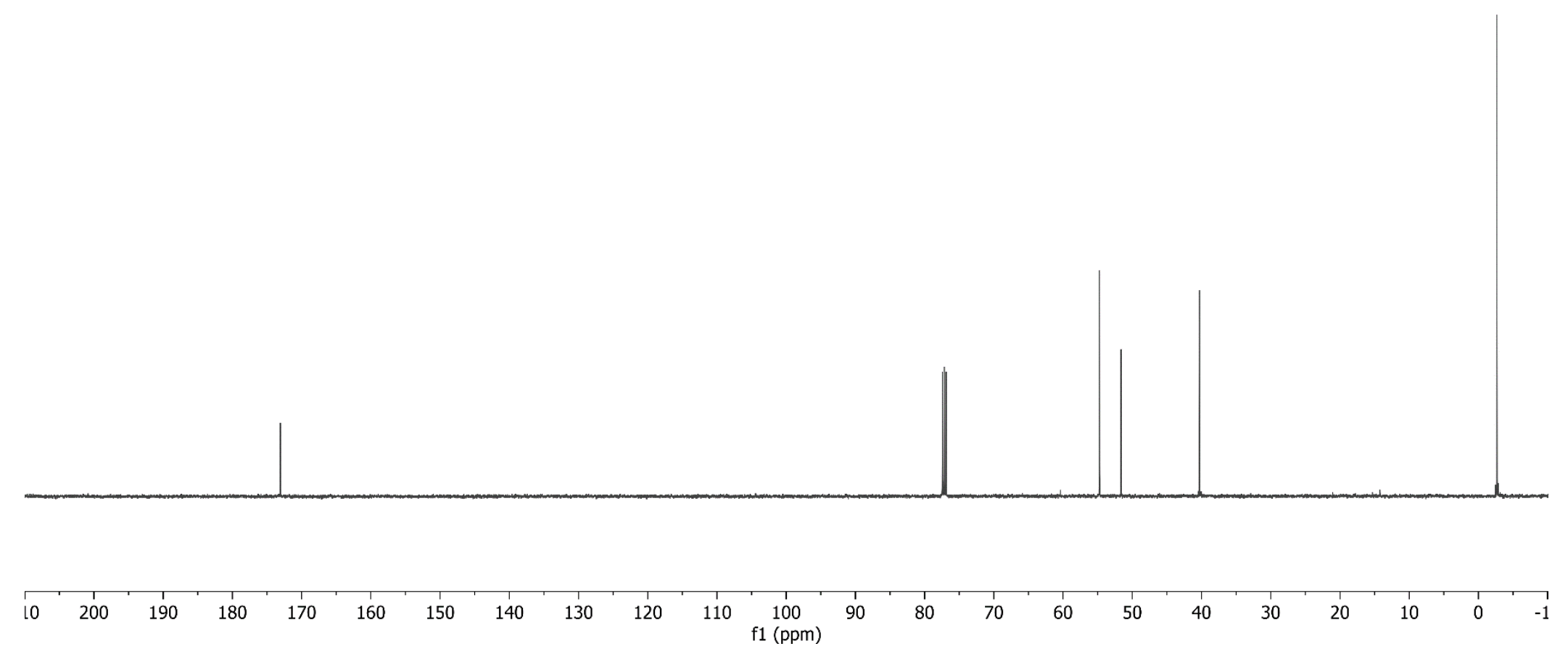


${ }^{1} \mathrm{H}$ NMR $\left(500 \mathrm{MHz}, \mathrm{CDCl}_{3}\right)$ spectrum of $\mathbf{1 g}$

Glu_TMS_H

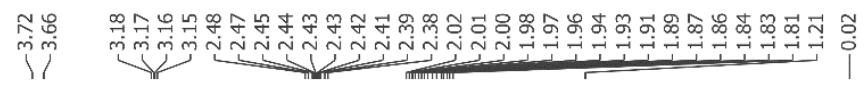
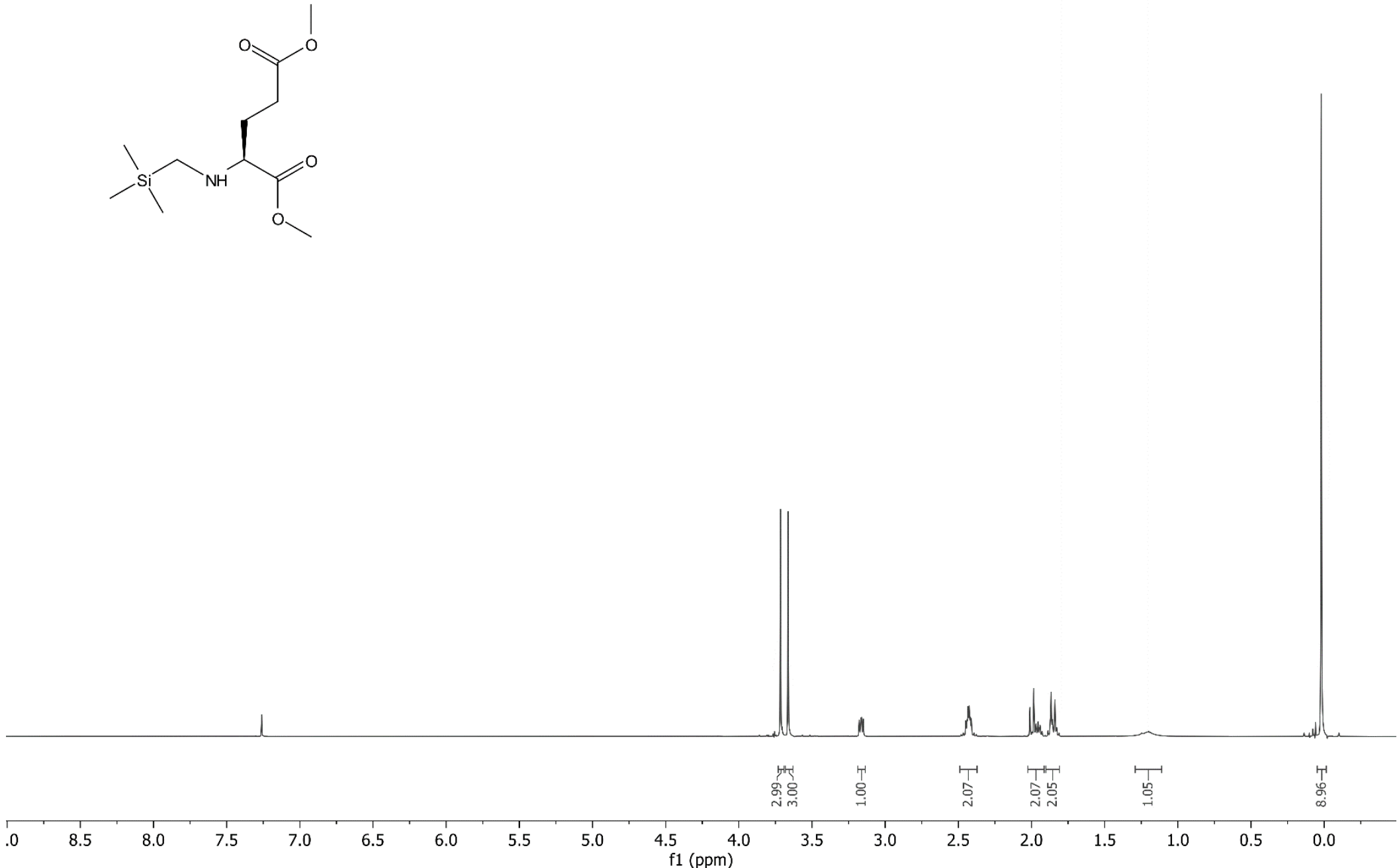
${ }^{13} \mathrm{C} \mathrm{NMR} \mathrm{(126} \mathrm{MHz,} \mathrm{CDCl}_{3}$ ) spectrum of $\mathbf{1 g}$

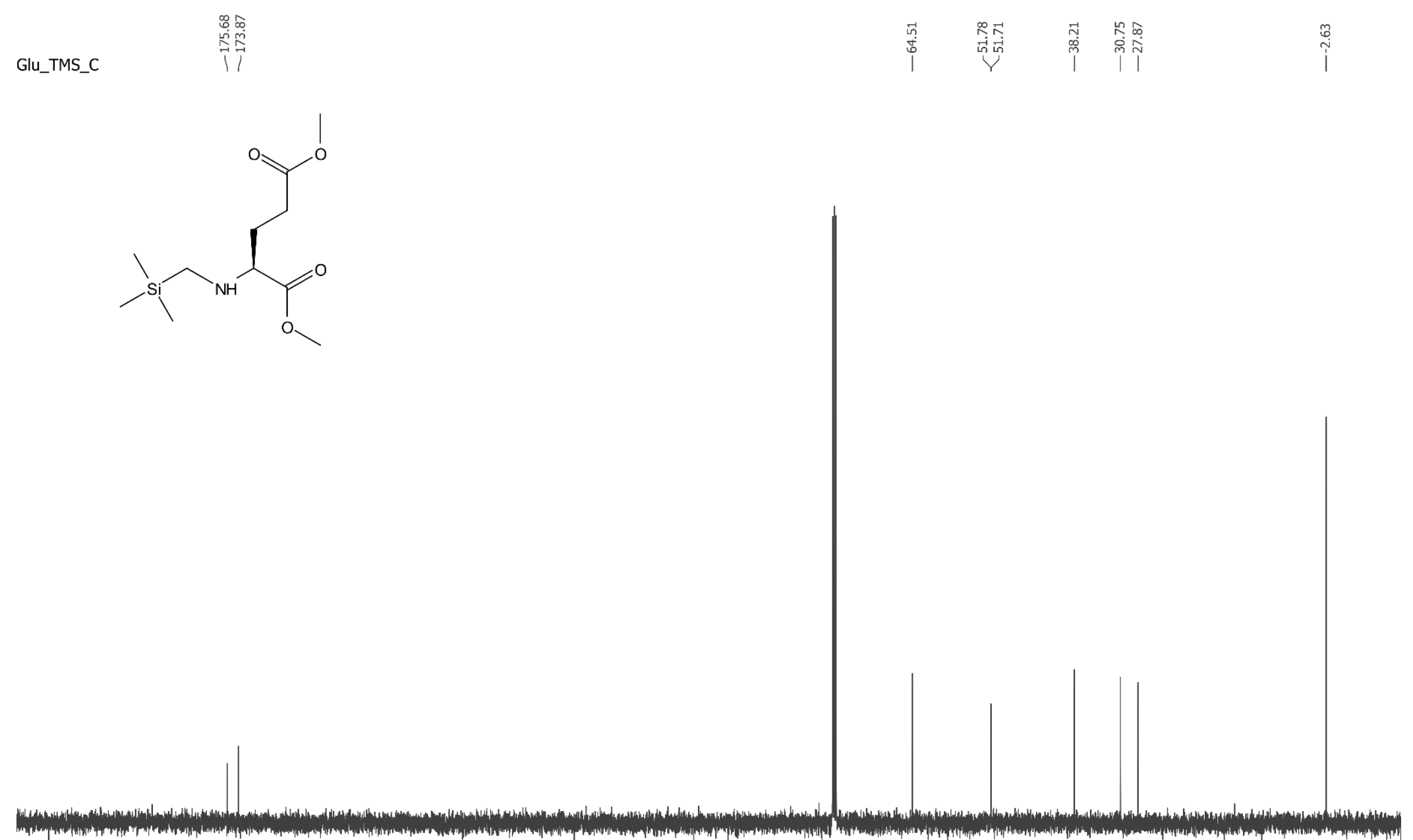

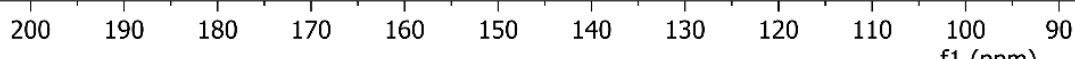


${ }^{1} \mathrm{H}$ NMR $\left(600 \mathrm{MHz}, \mathrm{CDCl}_{3}\right)$ spectrum of $\mathbf{1 h}$

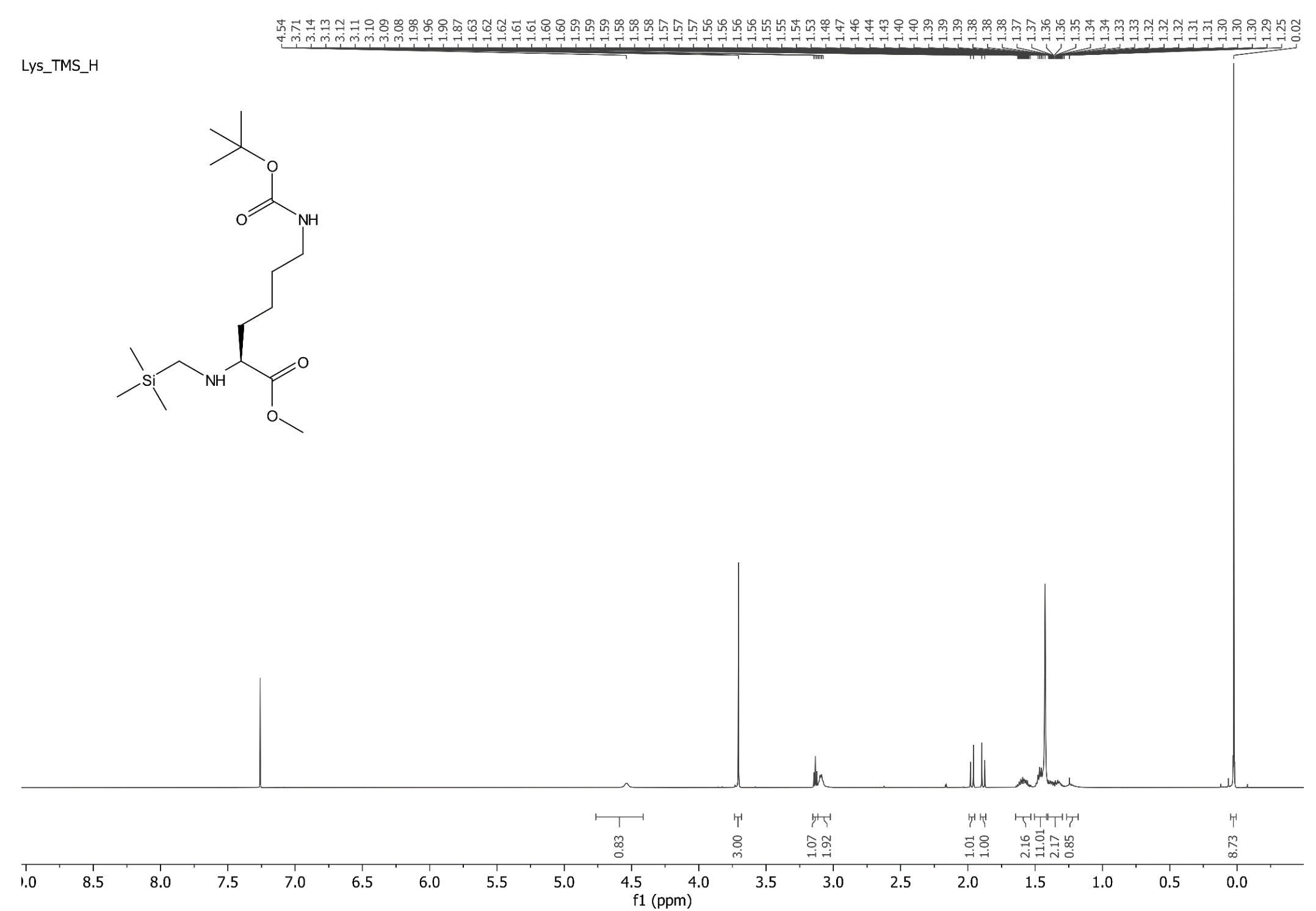


SUPPORTING INFORMATION

${ }^{13} \mathrm{C}$ NMR $\left(151 \mathrm{MHz}, \mathrm{CDCl}_{3}\right)$ spectrum of $\mathbf{1 h}$
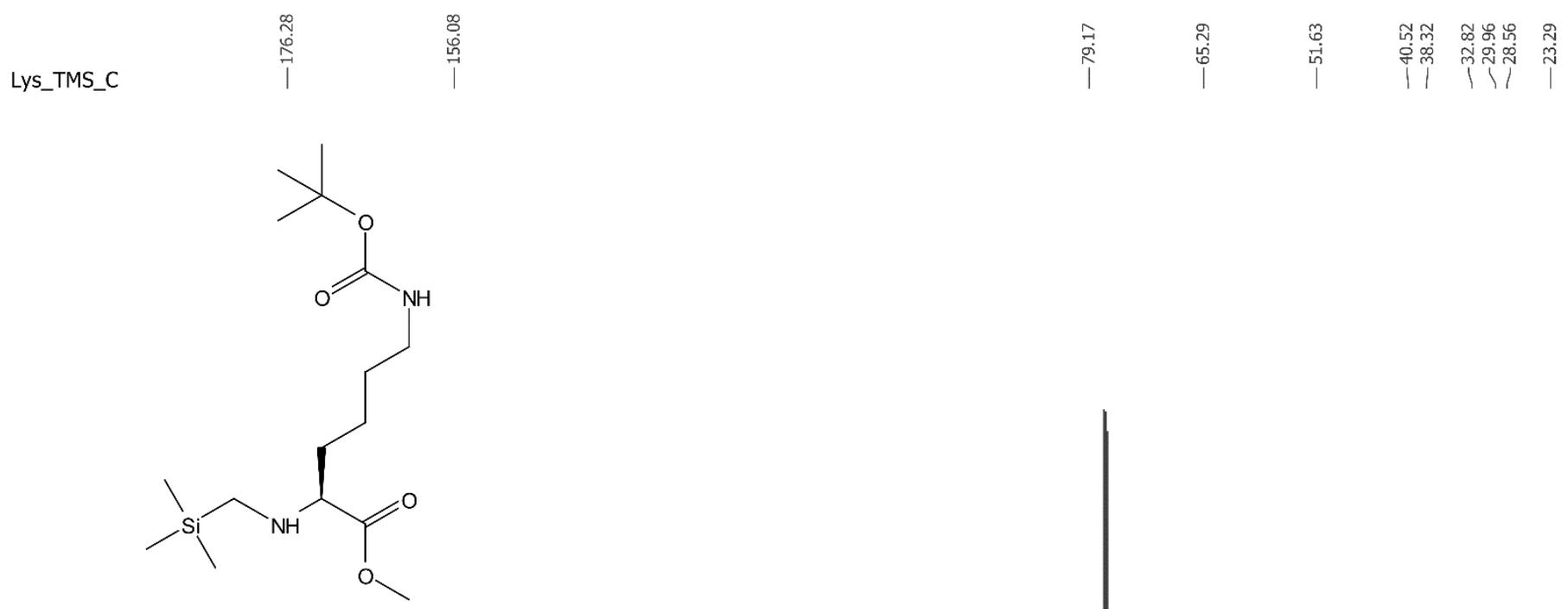

$\stackrel{\leftrightarrow}{\mathfrak{i}}$

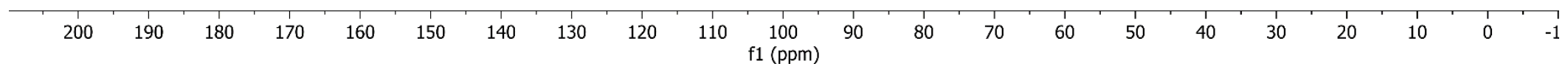

84 
${ }^{1} \mathrm{H} \mathrm{NMR}\left(500 \mathrm{MHz}, \mathrm{CDCl}_{3}\right)$ spectrum of $\mathbf{1 i}$

Piperadine_TMS_H

每

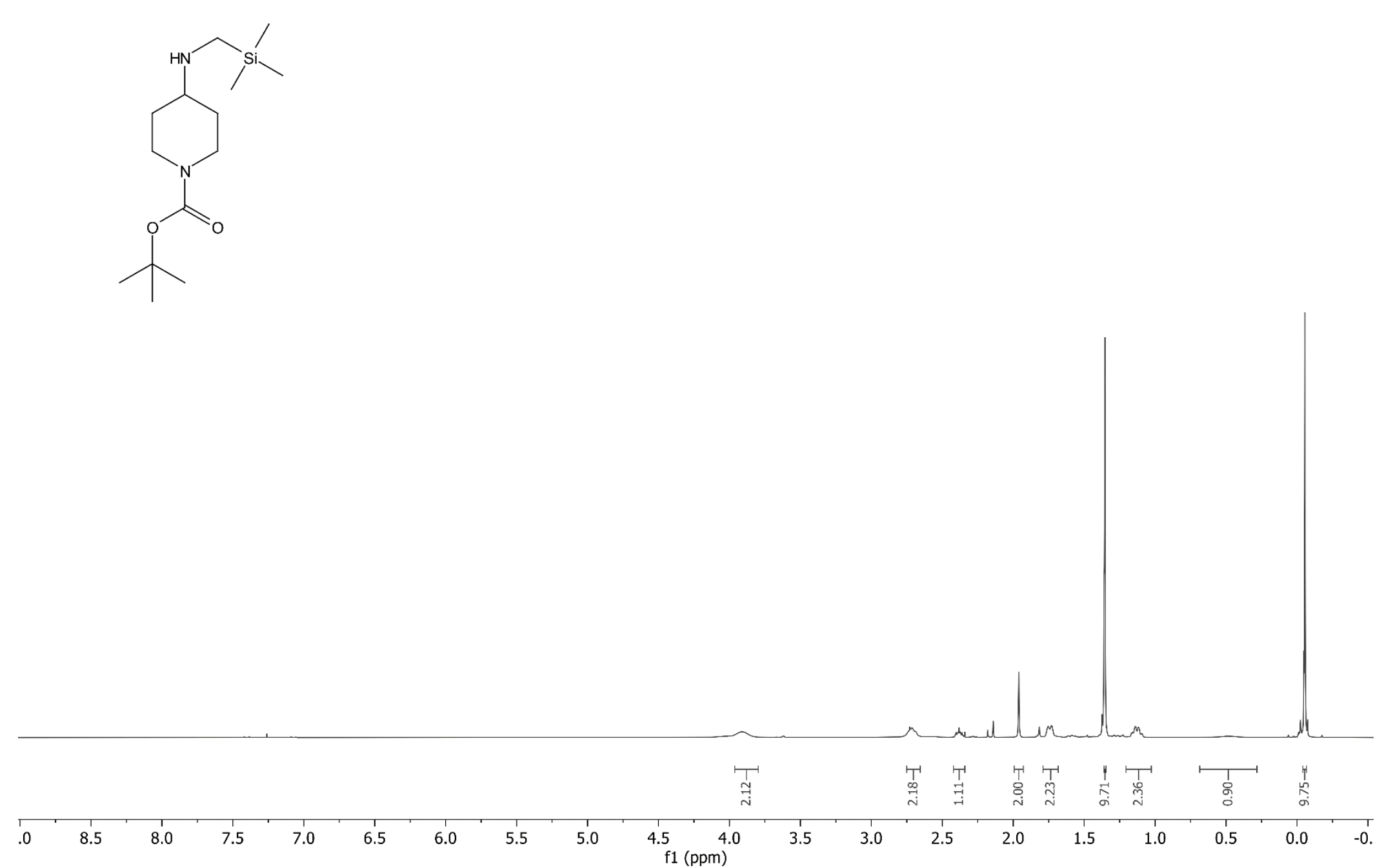


${ }^{13} \mathrm{C}$ NMR $\left(126 \mathrm{MHz}, \mathrm{CDCl}_{3}\right)$ spectrum of $1 \mathbf{i}$

Piperadine_TMS_C


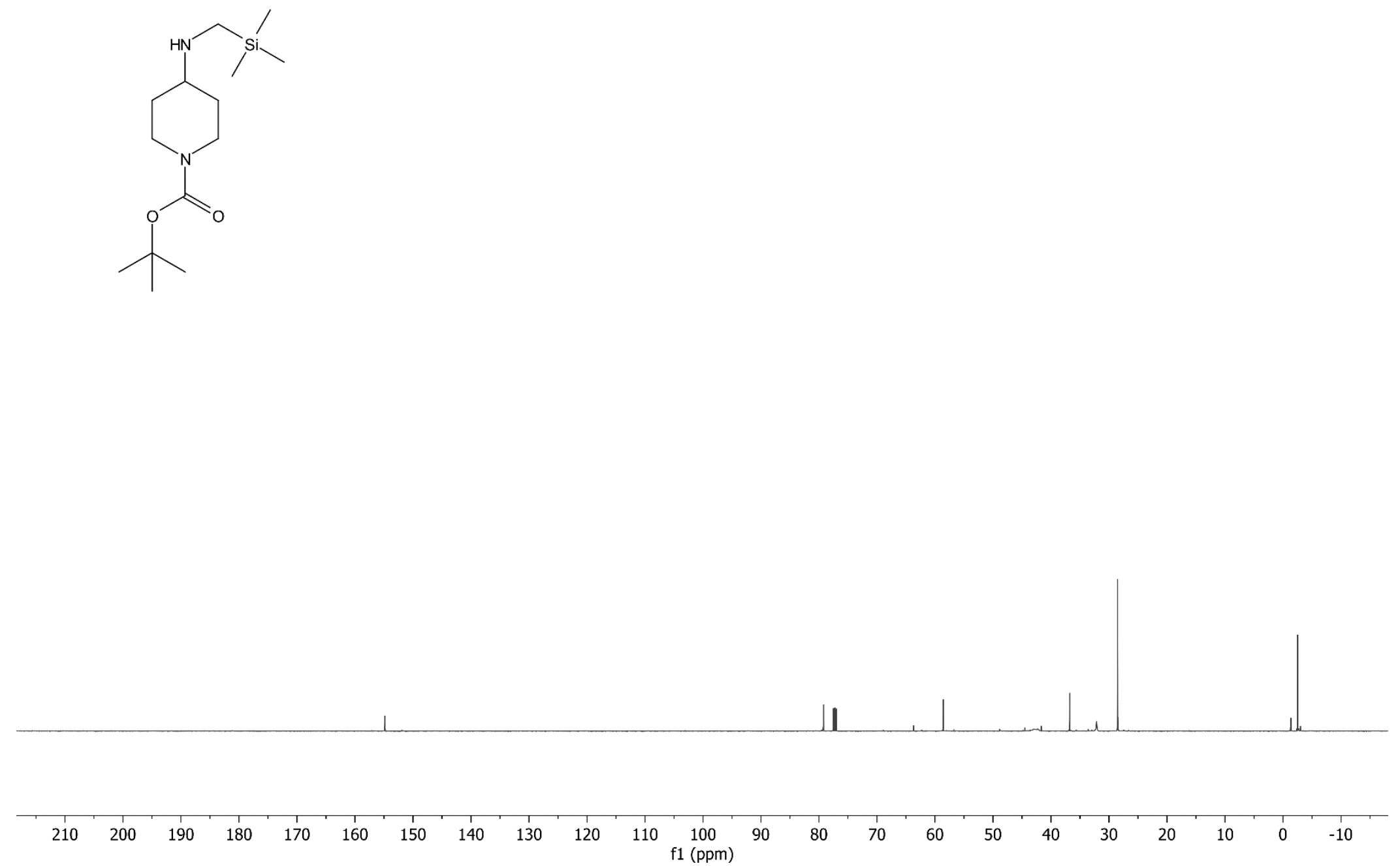


\section{${ }^{1} \mathrm{H} \mathrm{NMR}\left(500 \mathrm{MHz}, \mathrm{CDCl}_{3}\right)$ spectrum of $\mathbf{1 j}$}

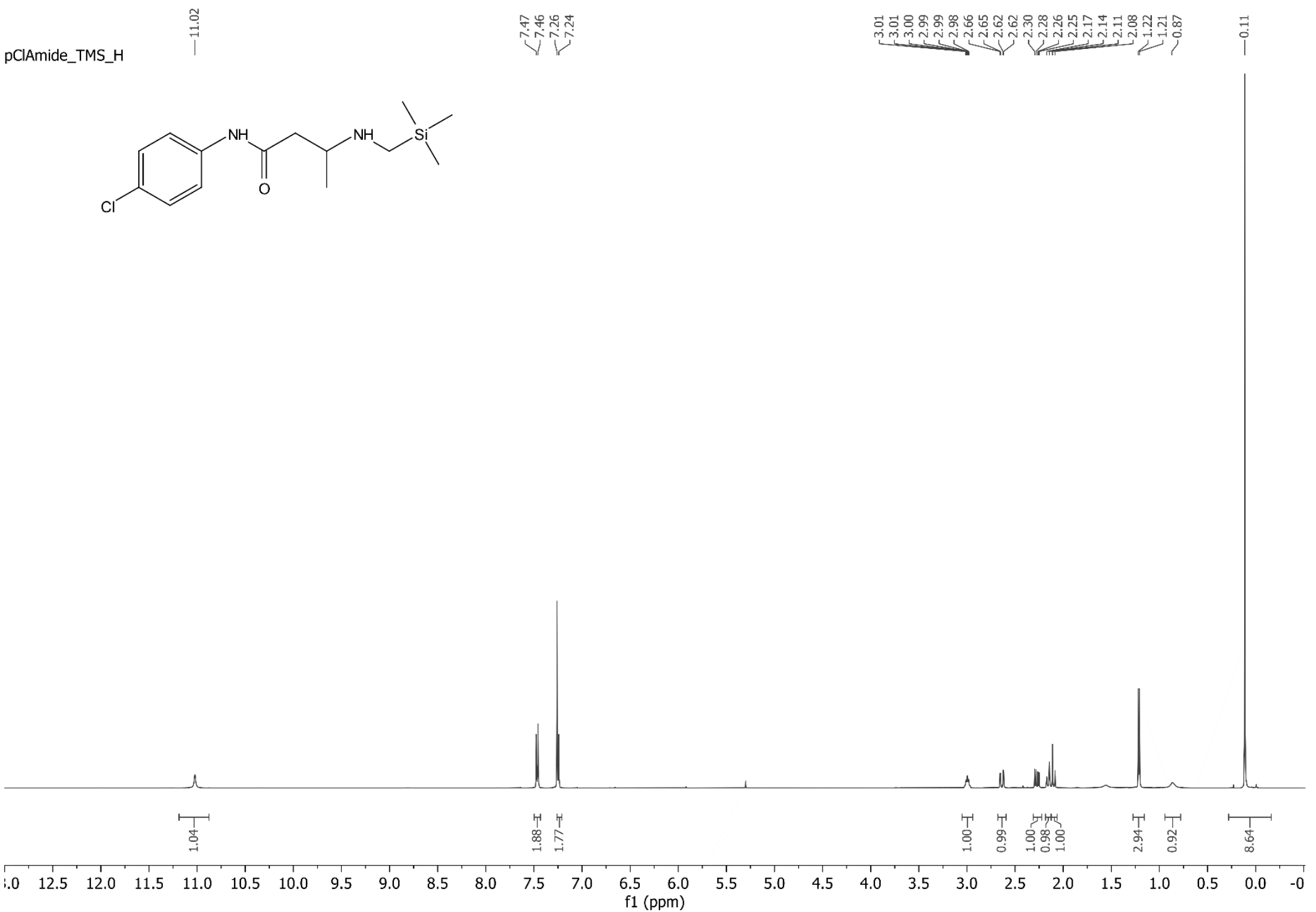


SUPPORTING INFORMATION

${ }^{13} \mathrm{C} \mathrm{NMR}\left(126 \mathrm{MHz}, \mathrm{CDCl}_{3}\right)$ spectrum of $\mathbf{1 j}$

pClAmide_TMS_C

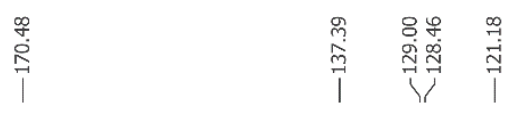

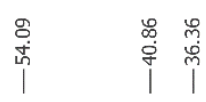

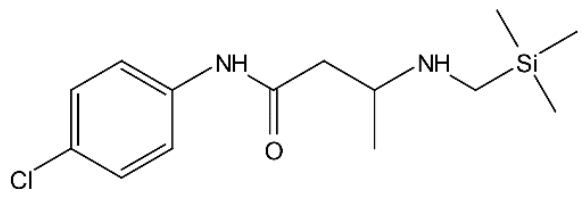

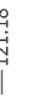

|

$\stackrel{i}{i}$

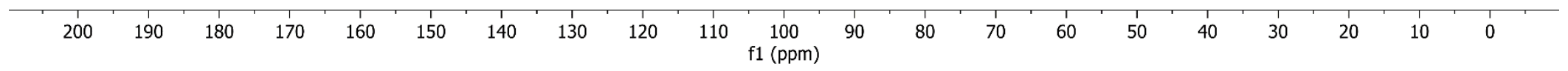

88 


\section{${ }^{1} \mathrm{H}$ NMR $\left(500 \mathrm{MHz}, \mathrm{CDCl}_{3}\right)$ spectrum of $\mathbf{1} \mathbf{k}$}

Sugar_TMS_H
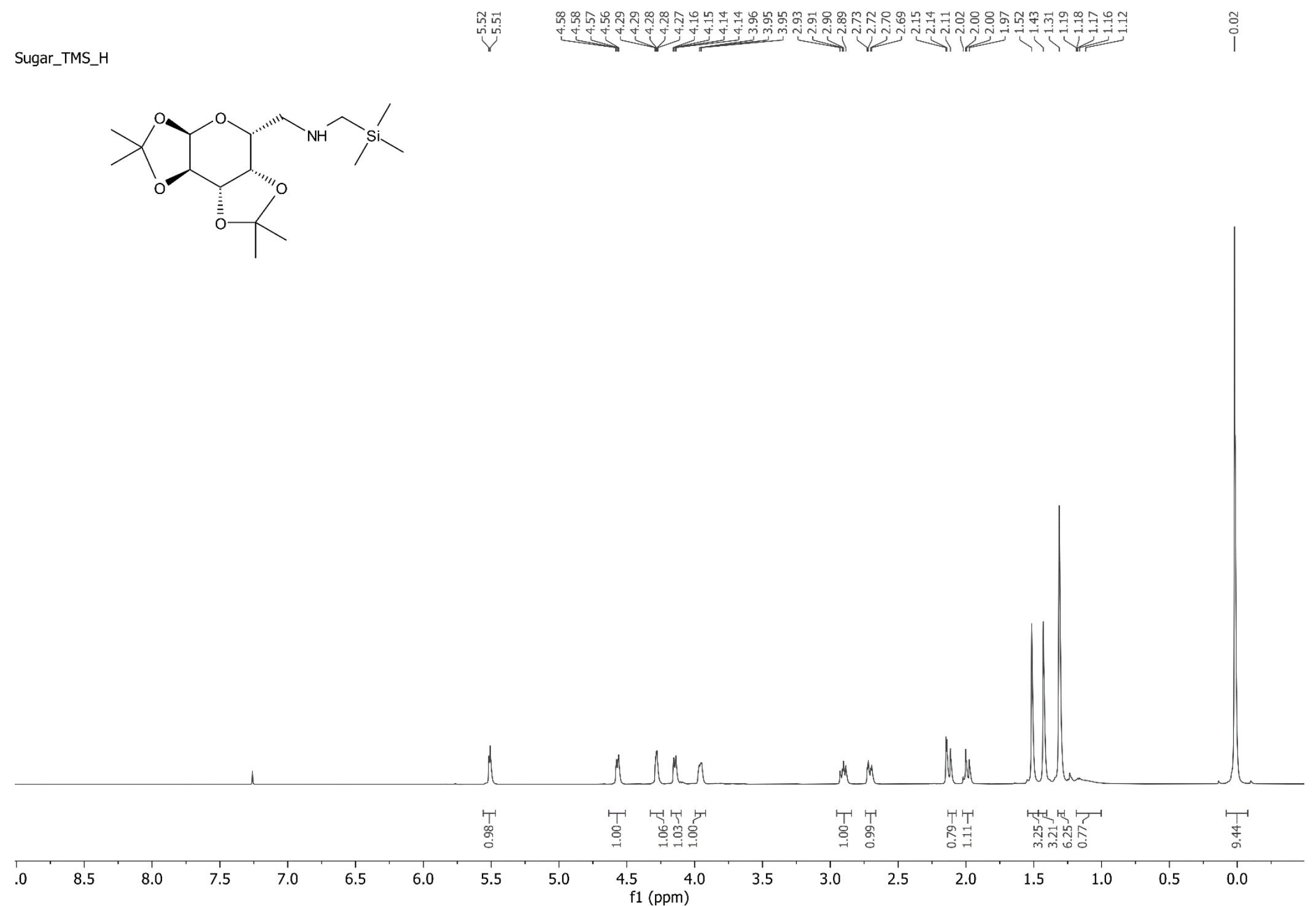
$\left.{ }^{13} \mathrm{C} \mathrm{NMR} \mathrm{(126} \mathrm{MHz,} \mathrm{CDCl}_{3}\right)$ spectrum of $\mathbf{1 k}$

Sugar_TMS_C

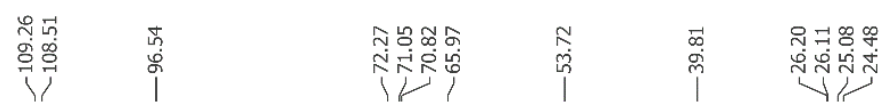

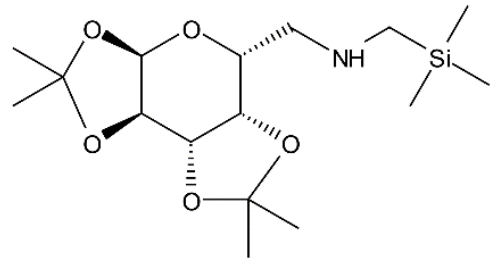

10

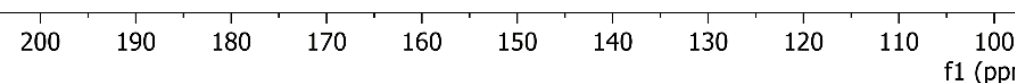

f1 100

80

60

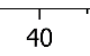

$20 \quad 10$ 
${ }^{1} \mathrm{H}$ NMR $\left(500 \mathrm{MHz}, \mathrm{CDCl}_{3}\right)$ spectrum of 11

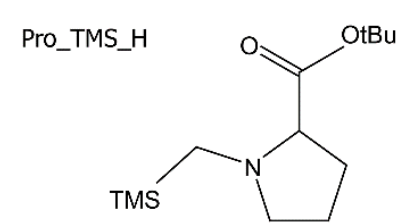

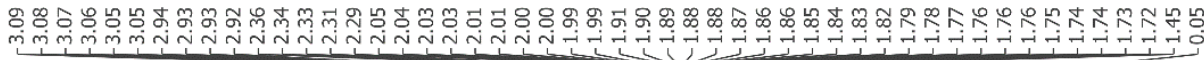

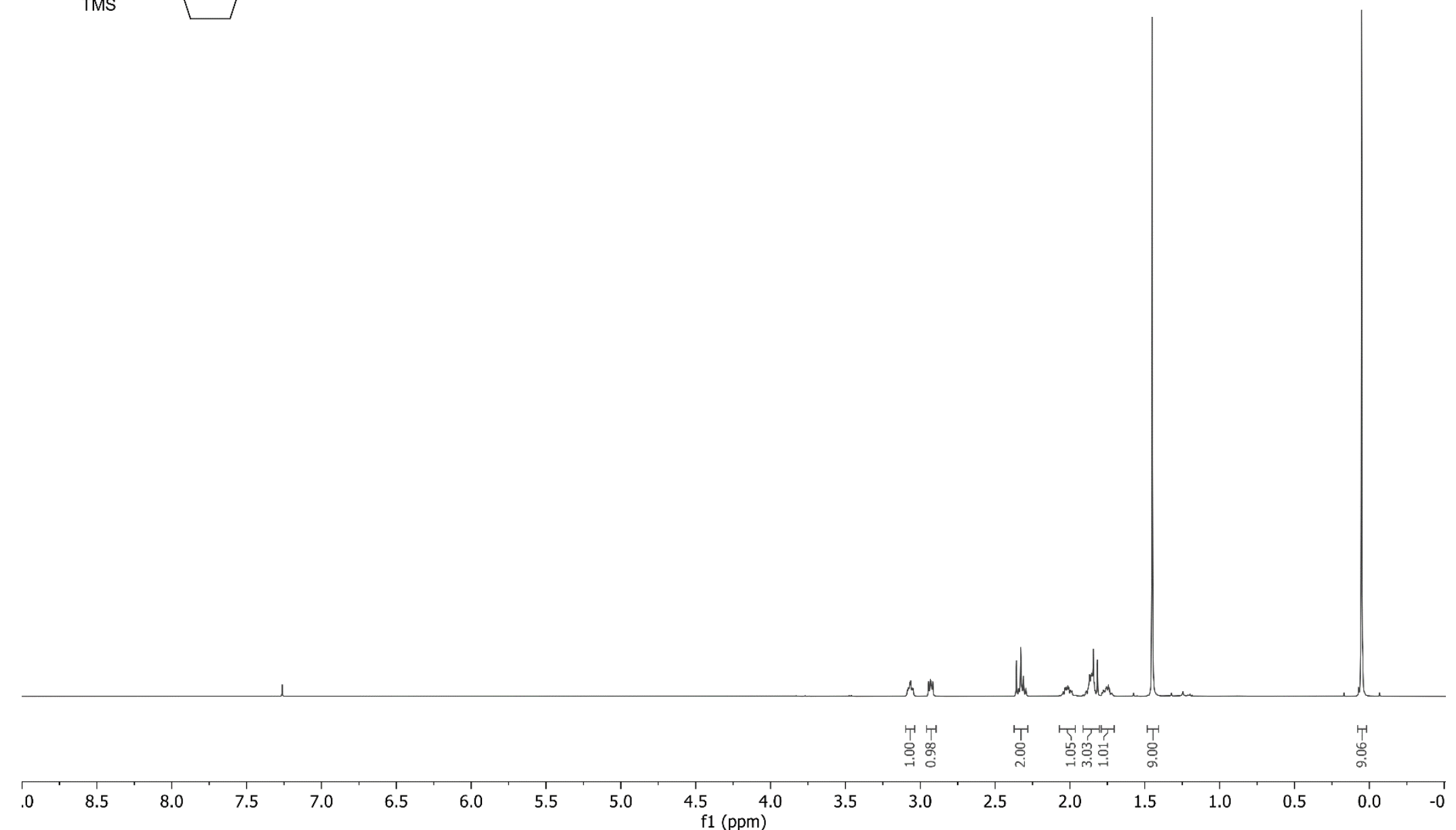


SUPPORTING INFORMATION

${ }^{13} \mathrm{C}$ NMR (126 MHz, $\mathrm{CDCl}_{3}$ ) spectrum of 1 I
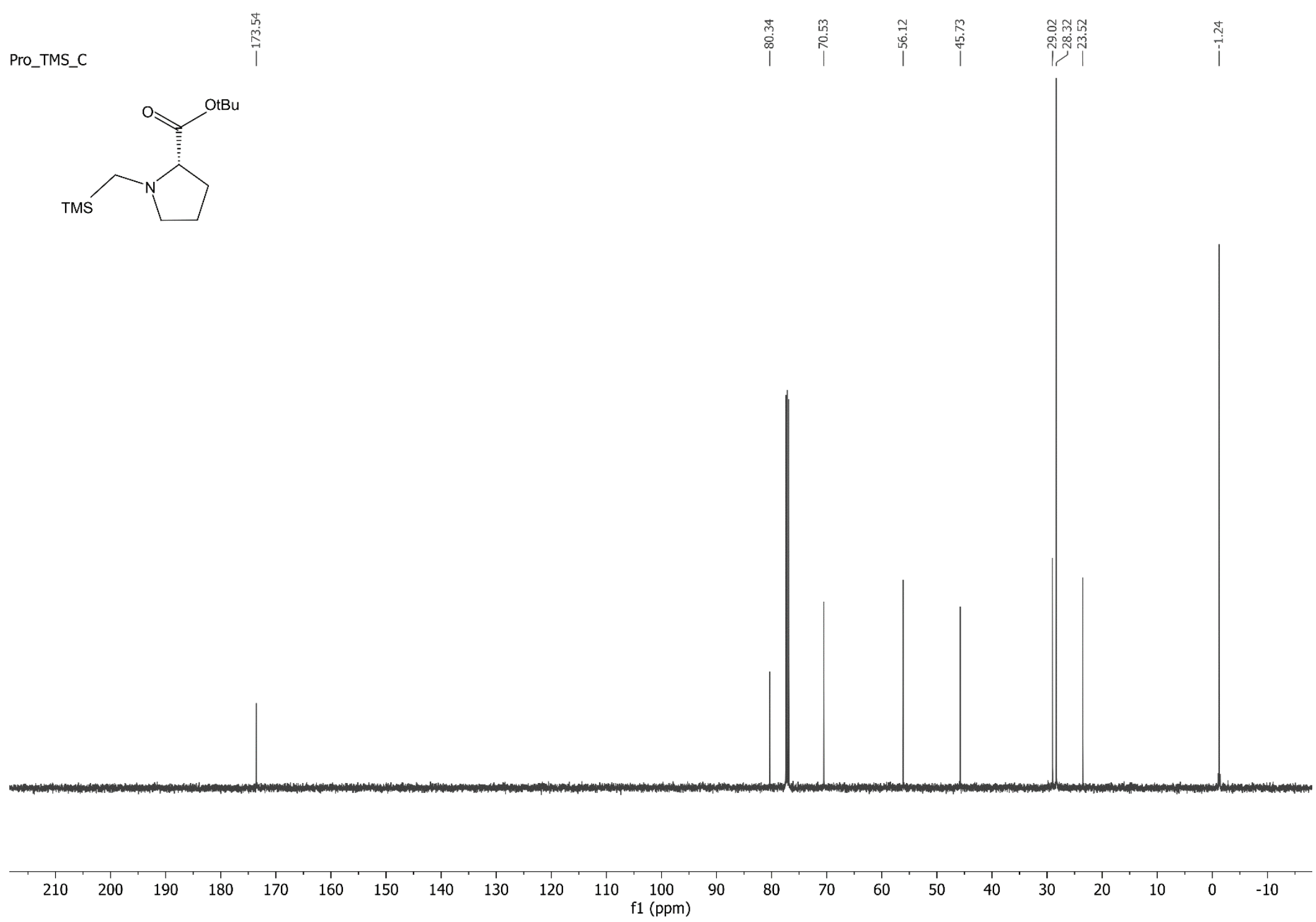

92 
${ }^{1} \mathrm{H}$ NMR $\left(400 \mathrm{MHz}, \mathrm{CDCl}_{3}\right)$ spectrum of $\mathbf{1 m}$

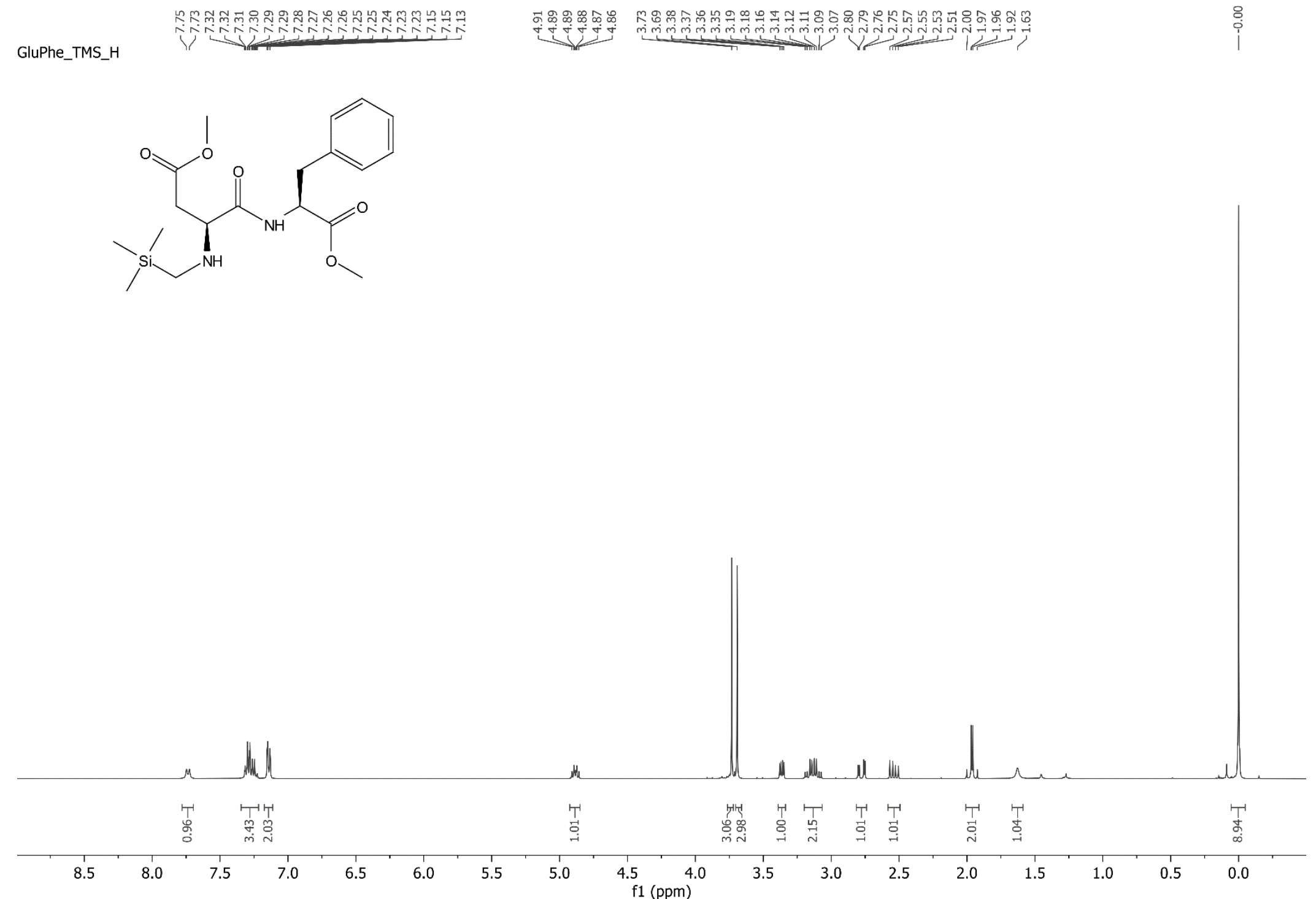


${ }^{13} \mathrm{C}$ NMR $\left(101 \mathrm{MHz}, \mathrm{CDCl}_{3}\right)$ spectrum of $\mathbf{1 m}$

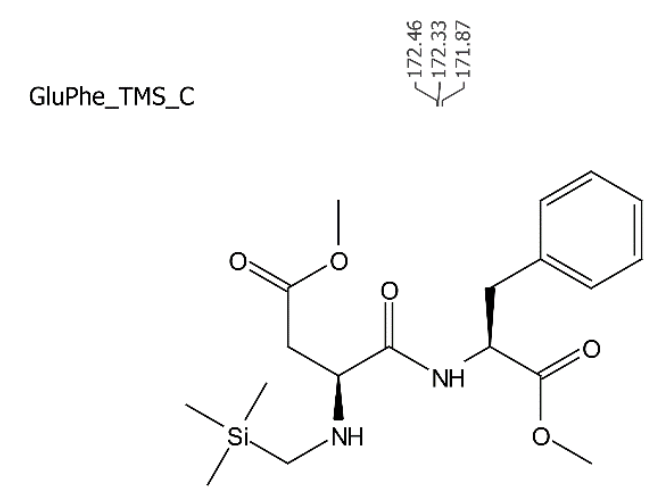

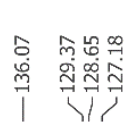

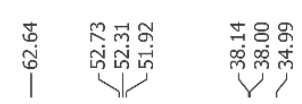

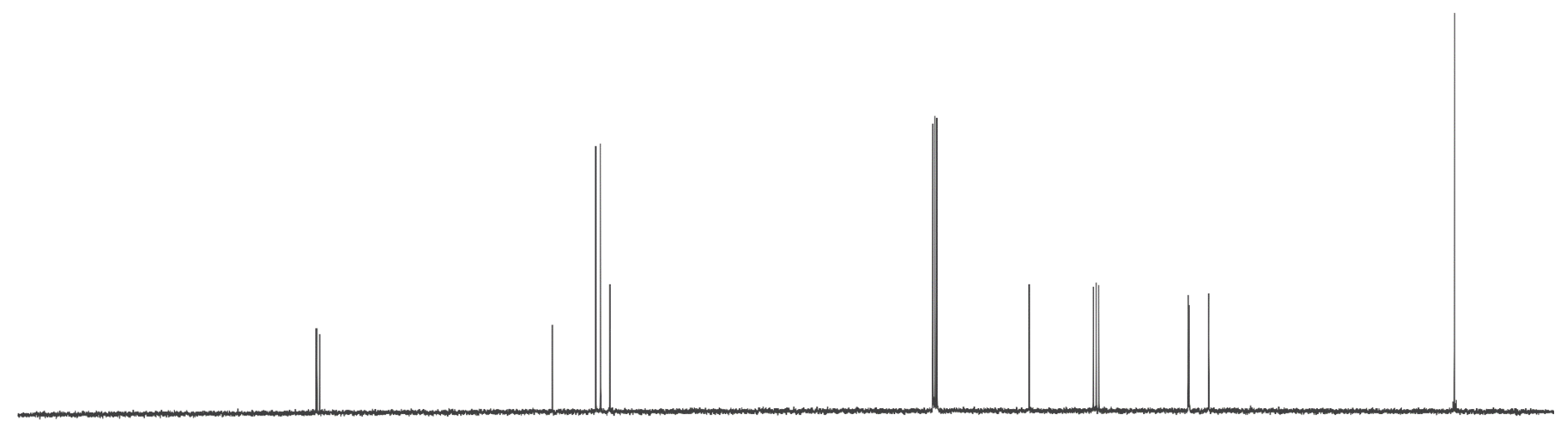


${ }^{1} \mathrm{H}$ NMR (400 MHz, $\left.\mathrm{CDCl}_{3}\right)$ spectrum of $\mathbf{1 n}$

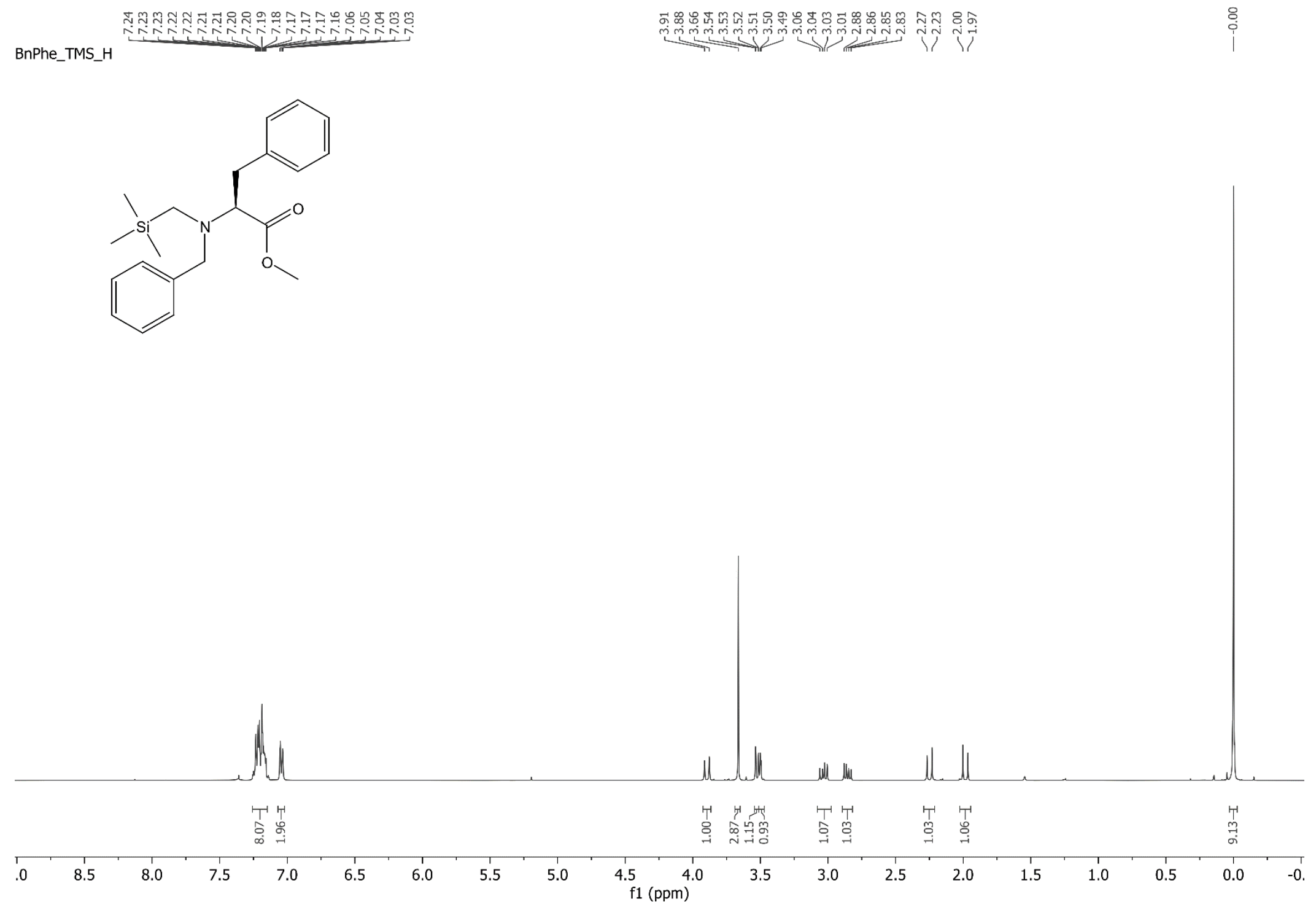


${ }^{13} \mathrm{C} \mathrm{NMR}\left(101 \mathrm{MHz}, \mathrm{CDCl}_{3}\right.$ ) spectrum of $\mathbf{1 n}$

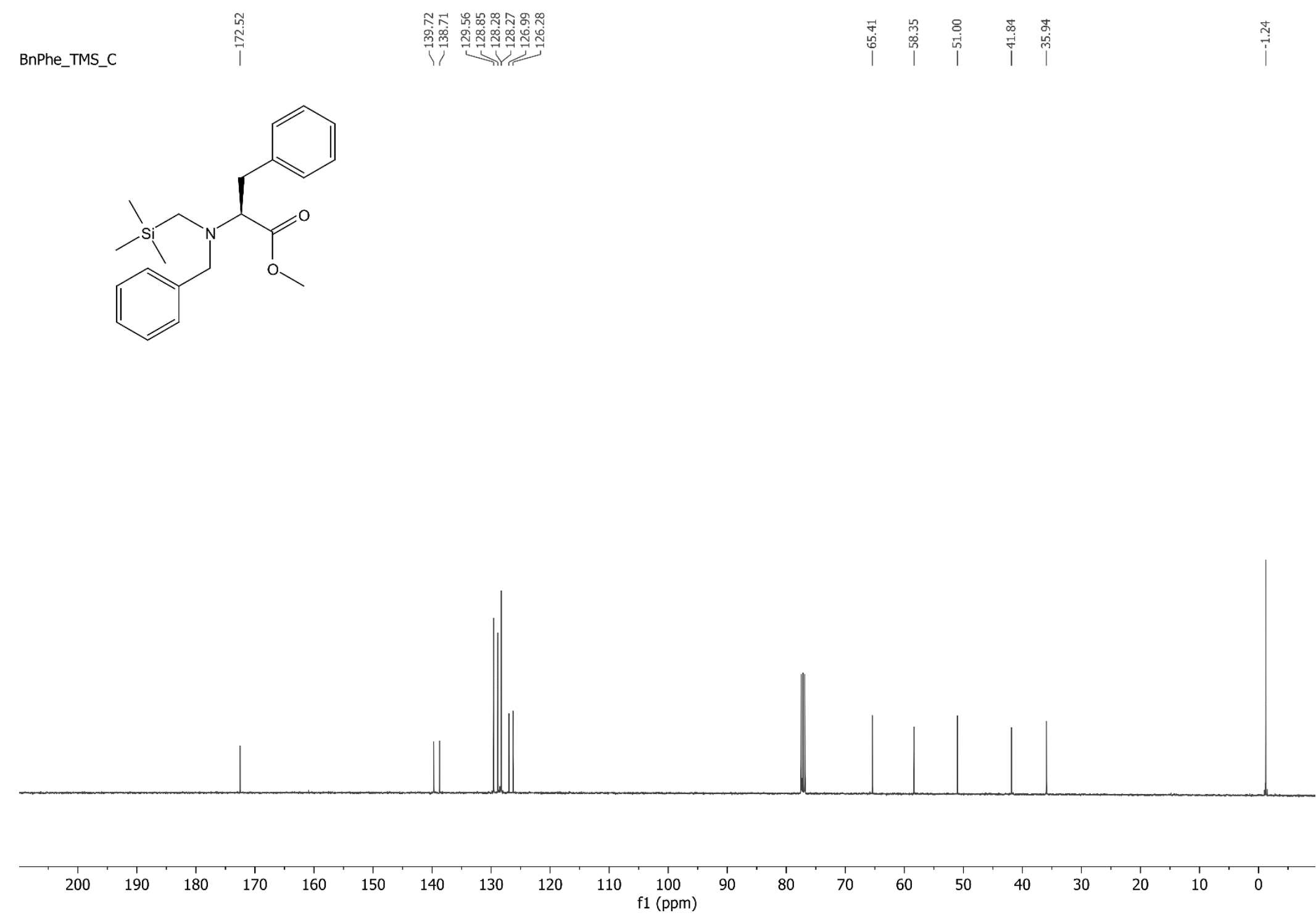


${ }^{1} \mathrm{H}$ NMR $\left(600 \mathrm{MHz}, \mathrm{CDCl}_{3}\right)$ spectrum of 10

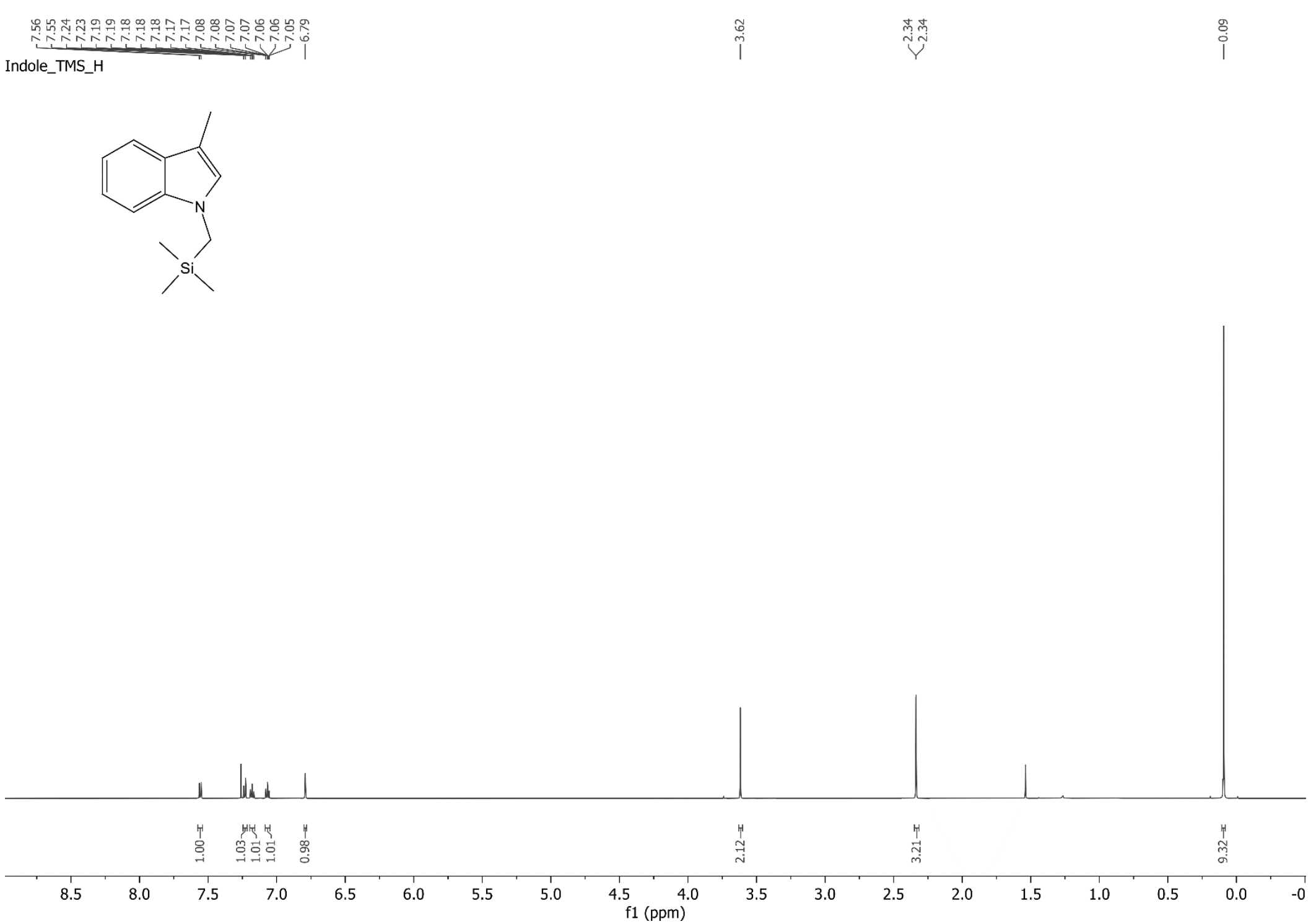


SUPPORTING INFORMATION

${ }^{13} \mathrm{C}$ NMR $\left(151 \mathrm{MHz}, \mathrm{CDCl}_{3}\right)$ spectrum of 10

Indole_TMS_C

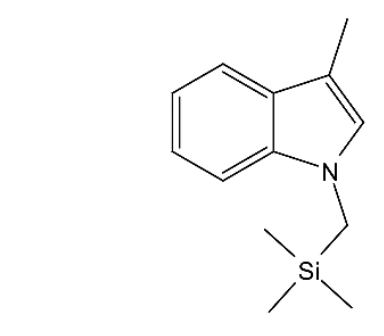

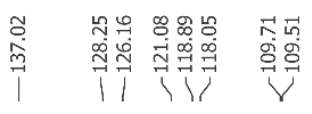

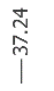

$\stackrel{\stackrel{ \pm}{m}}{\text { I }}$

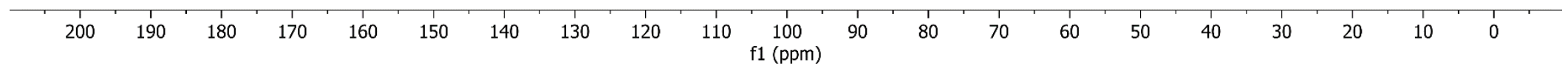

98 
${ }^{1} \mathrm{H}$ NMR $\left(400 \mathrm{MHz}, \mathrm{CDCl}_{3}\right)$ spectrum of $1 \mathrm{p}$

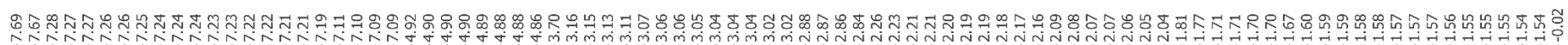
Phe-Pro-CH2TMS_H<smiles>COC(=O)[C@H](Cc1ccccc1)NC(=O)[C@@H]1CCCN1C[Si](C)(C)C</smiles>

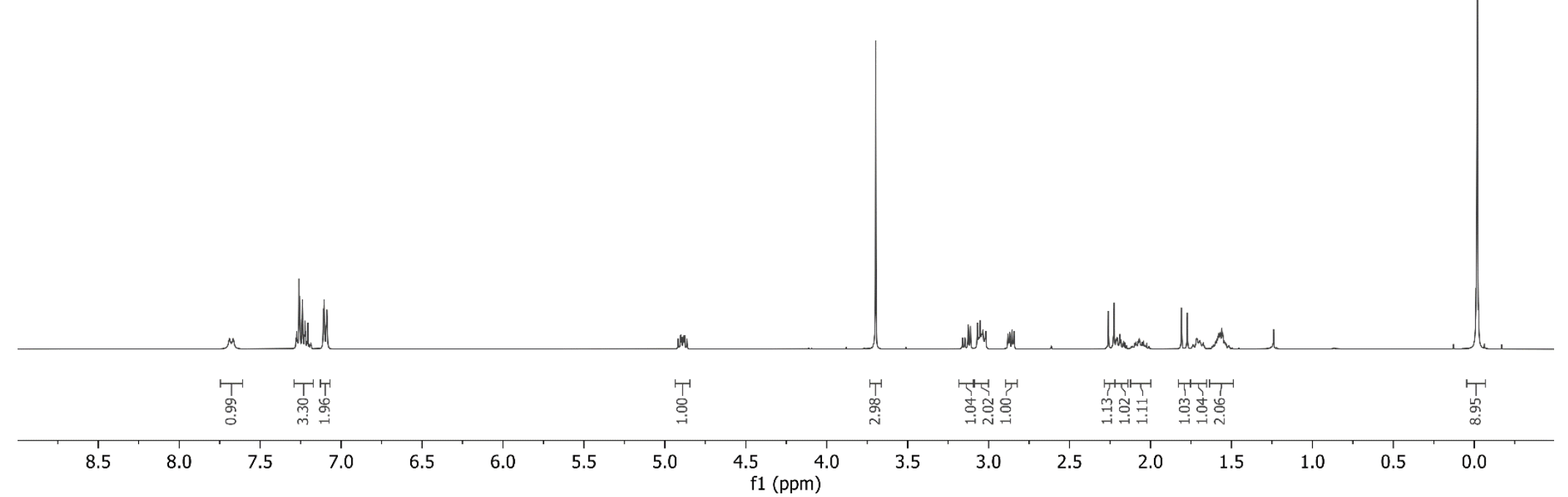


SUPPORTING INFORMATION

${ }^{13} \mathrm{C}$ NMR $\left(101 \mathrm{MHz}, \mathrm{CDCl}_{3}\right)$ spectrum of ip
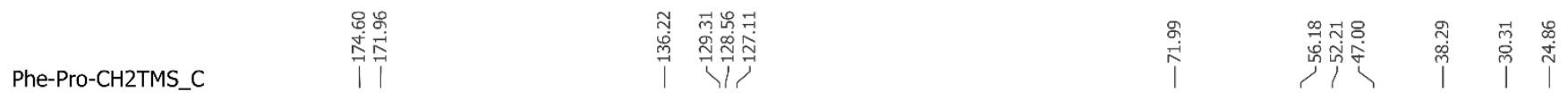

$\stackrel{\infty}{i}$
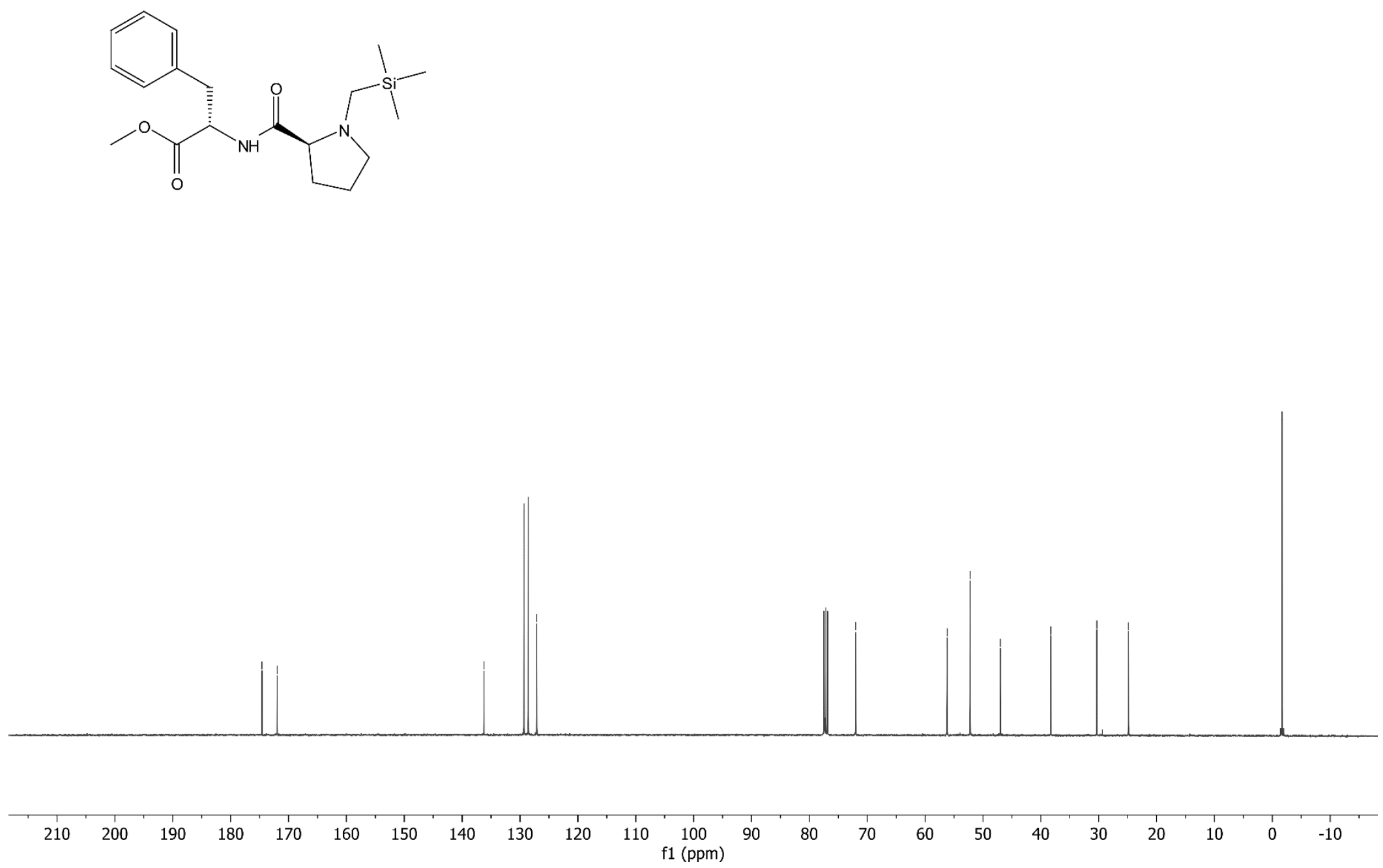

100 
${ }^{1} \mathrm{H}$ NMR $\left(500 \mathrm{MHz}, \mathrm{CDCl}_{3}\right)$ spectrum of $\mathbf{2 a}$

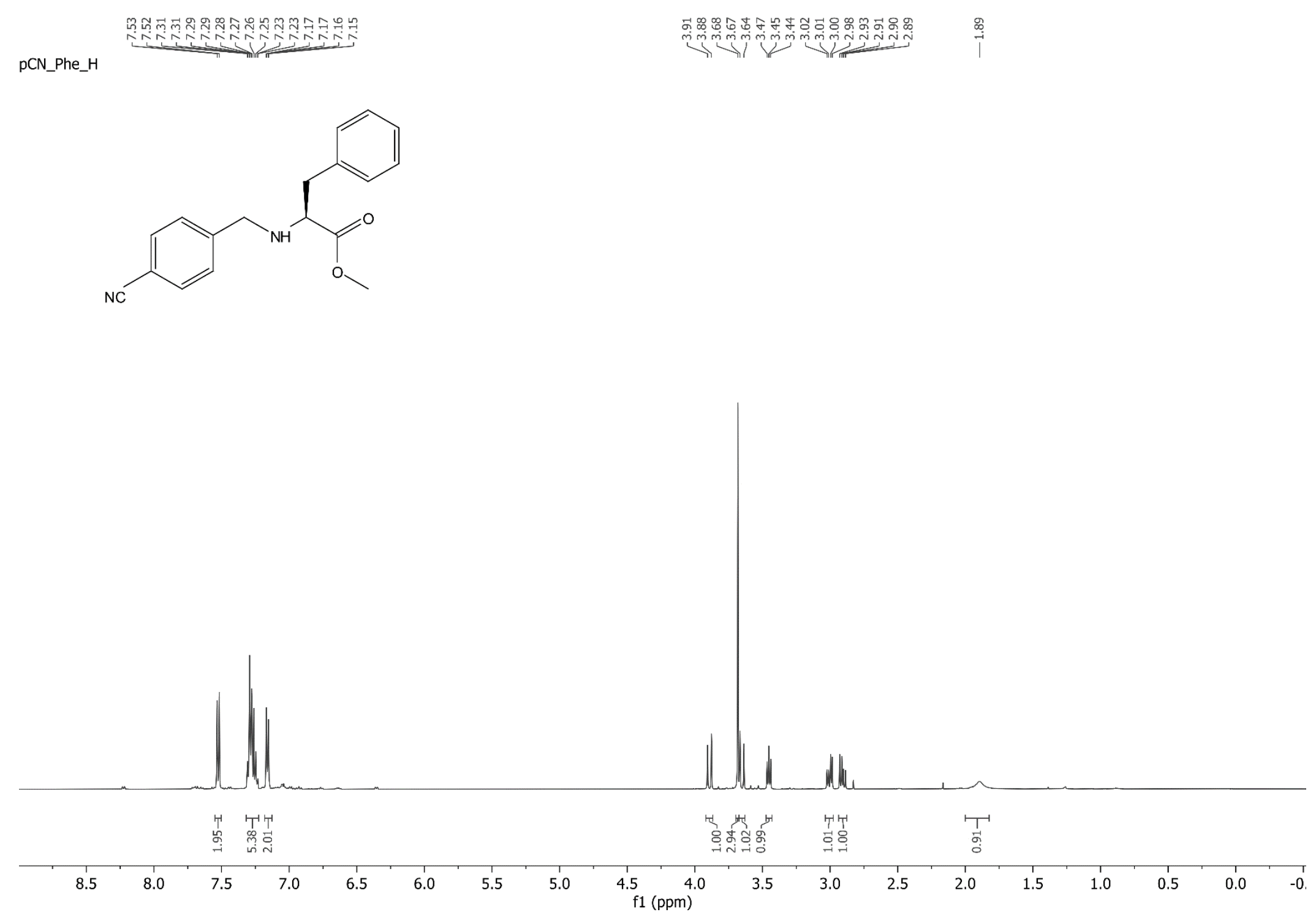


${ }^{13} \mathrm{C} \mathrm{NMR} \mathrm{(126} \mathrm{MHz,} \mathrm{CDCl}_{3}$ ) spectrum of $\mathbf{2 a}$

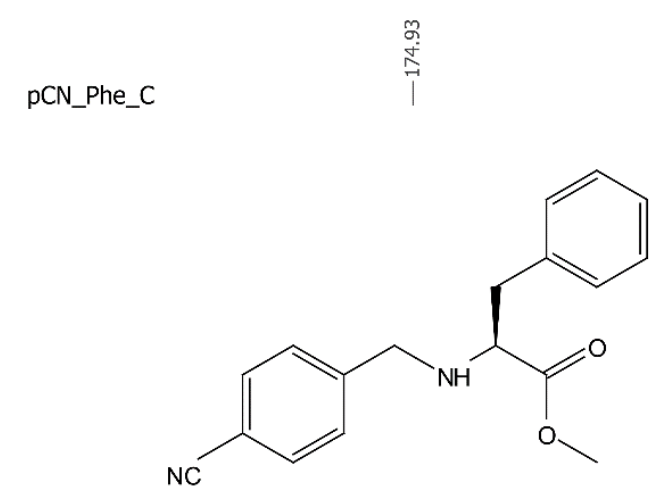

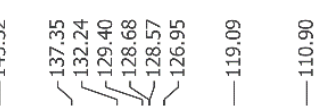

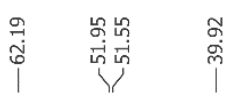

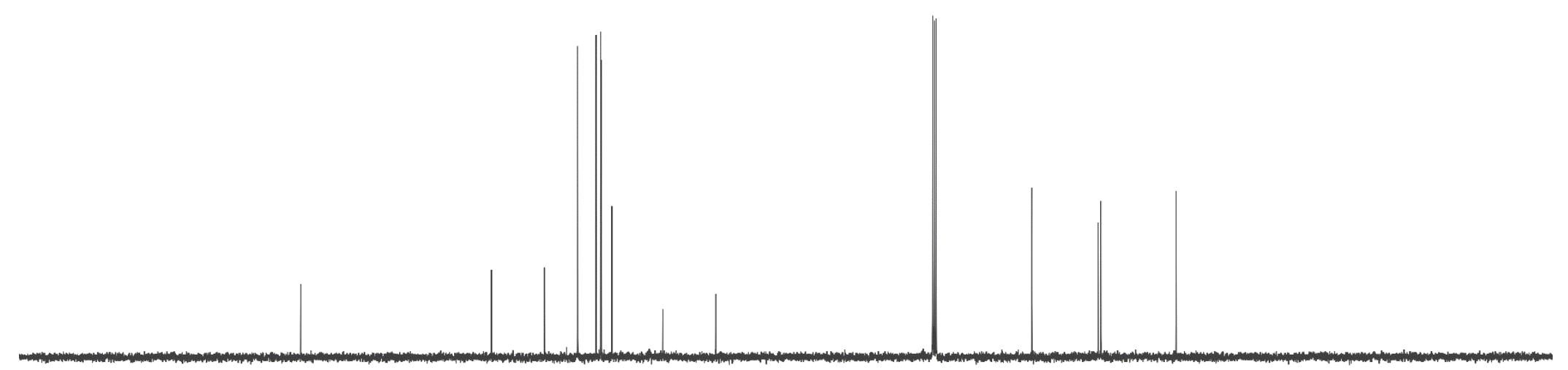


${ }^{1} \mathrm{H}$ NMR (500 MHz, CDCl 3 ) spectrum of 2b

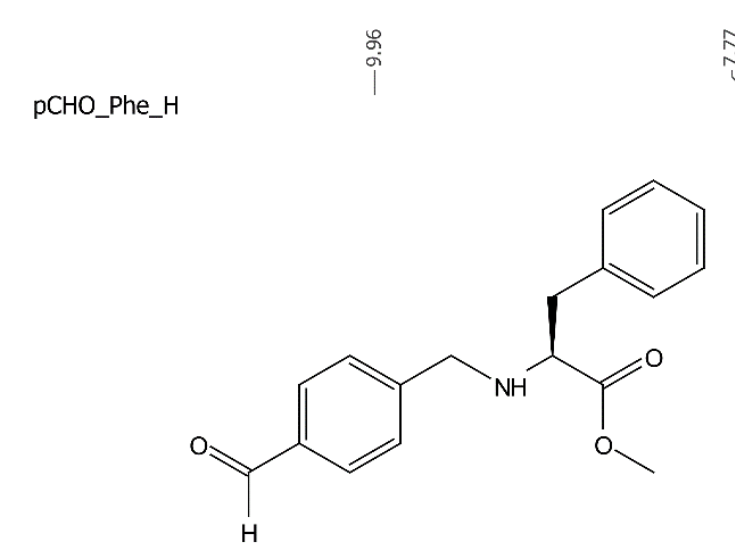

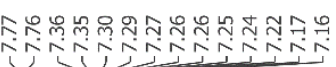

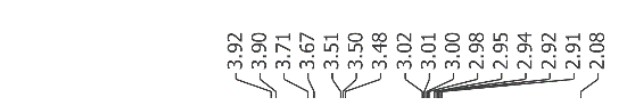

pCHO_Phe_H
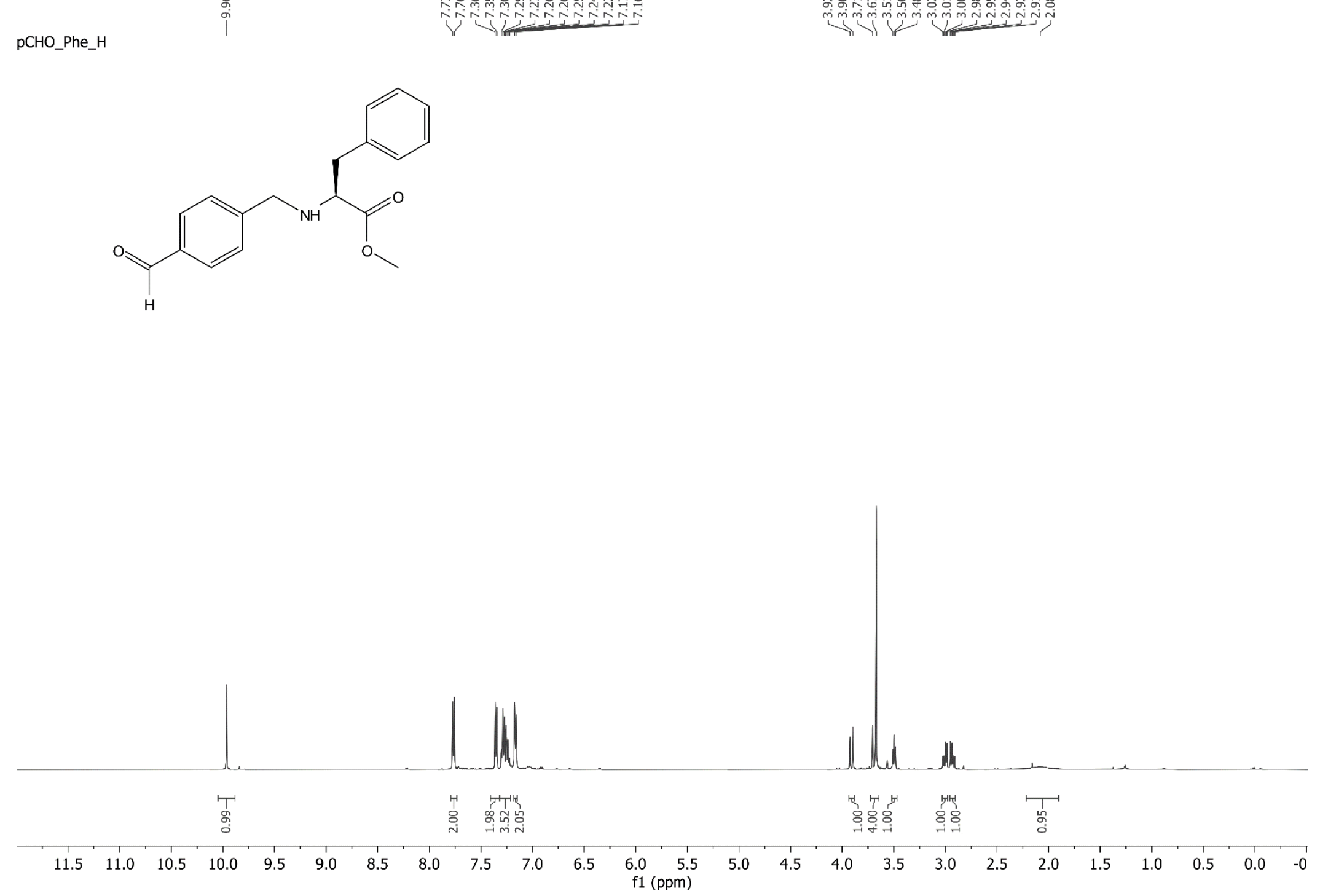
${ }^{13} \mathrm{C} \mathrm{NMR} \mathrm{(126} \mathrm{MHz,} \mathrm{CDCl}_{3}$ ) spectrum of $\mathbf{2 b}$
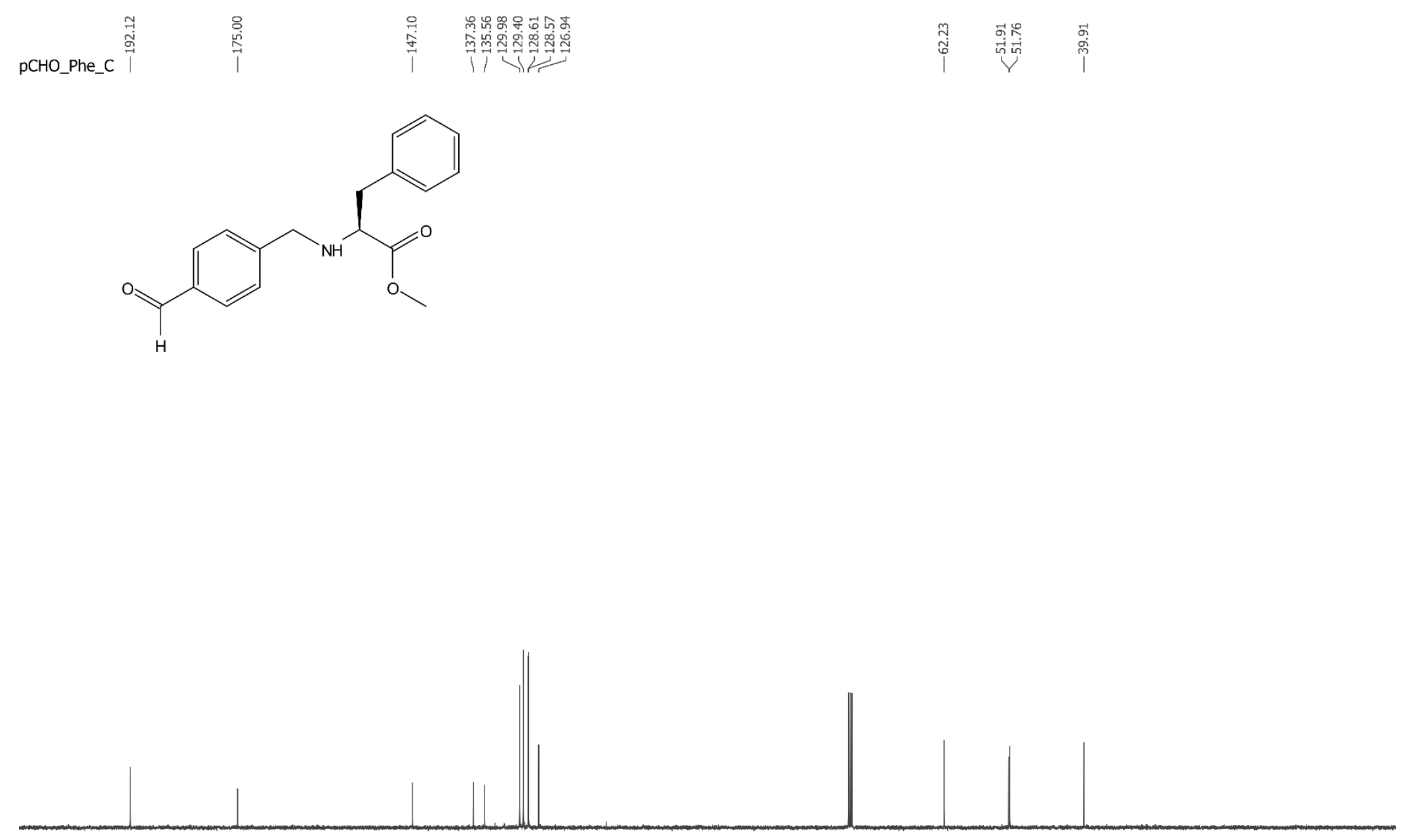

$\begin{array}{lllllllllllllllllllllll}200 & 190 & 180 & 170 & 160 & 150 & 140 & 130 & 120 & 110 & 100 & 90 & 80 & 70 & 60 & 50 & 40 & 30 & 20 & 10 & 0 & \end{array}$ 
${ }^{1} \mathrm{H}$ NMR $\left(600 \mathrm{MHz}, \mathrm{CDCl}_{3}\right)$ spectrum of $2 \mathrm{c}$

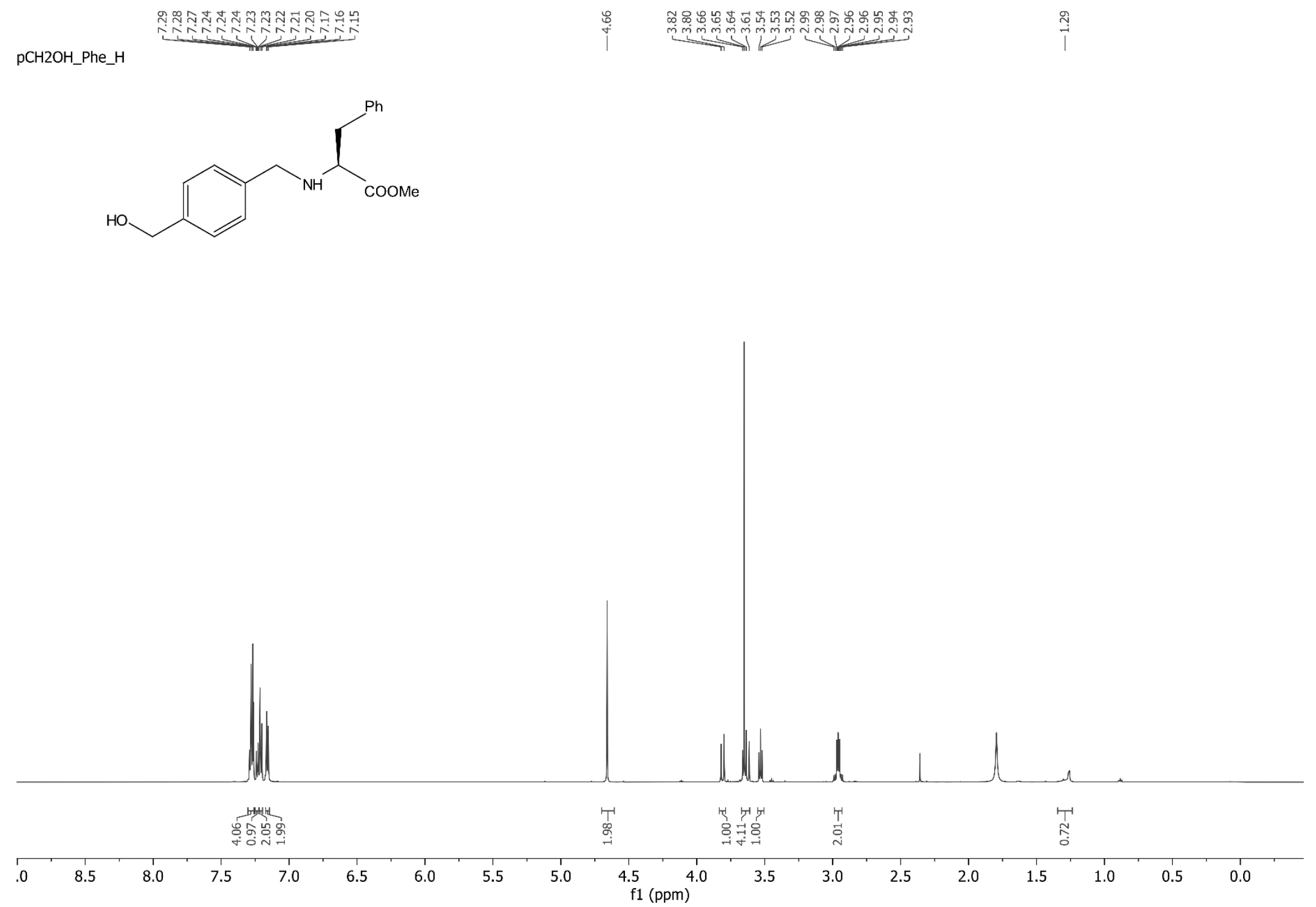


${ }^{13} \mathrm{C}$ NMR $\left(151 \mathrm{MHz}, \mathrm{CDCl}_{3}\right)$ spectrum of 2c

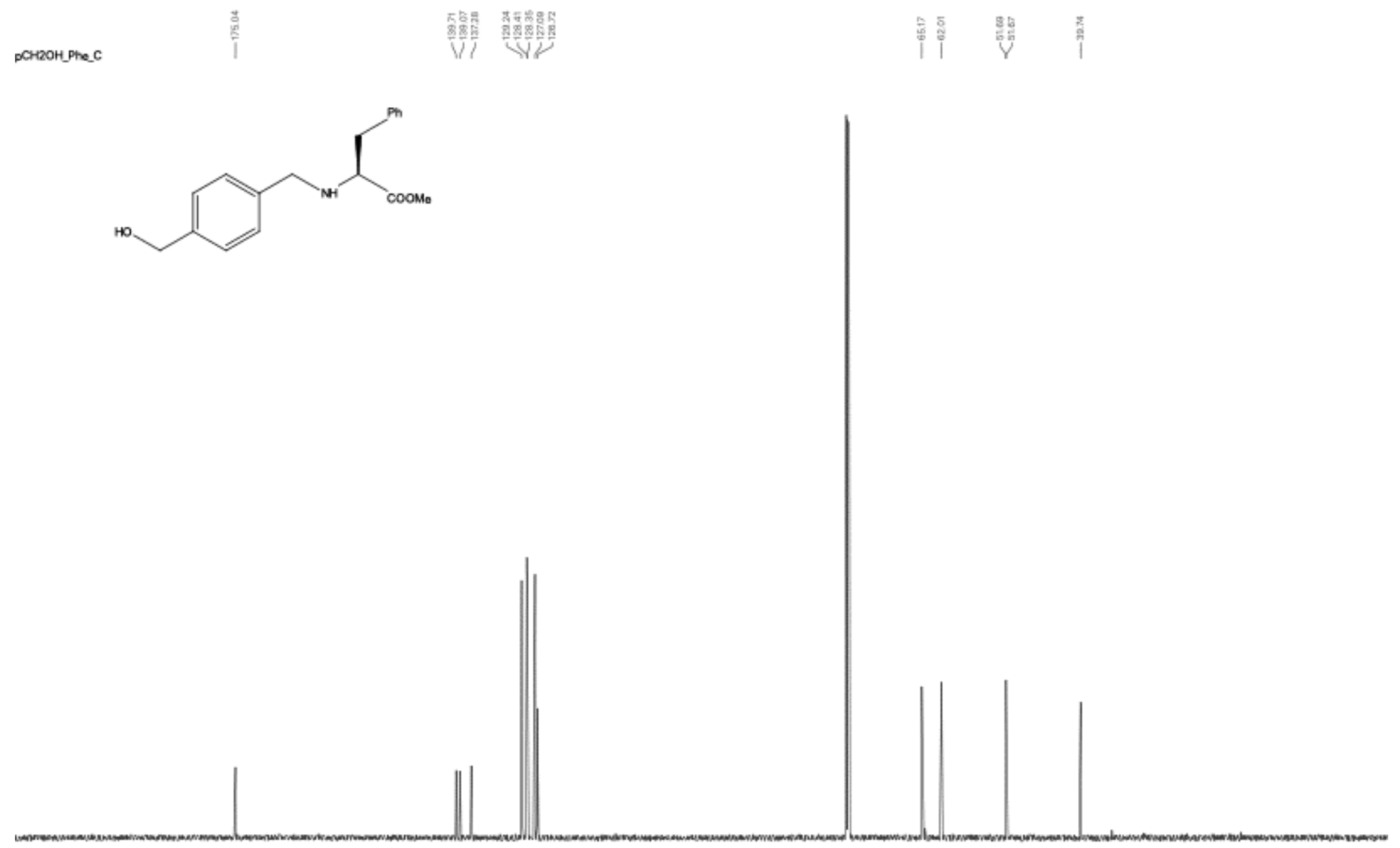

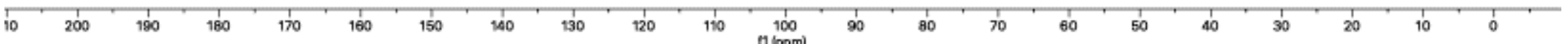


${ }^{1} \mathrm{H}$ NMR $\left(500 \mathrm{MHz}, \mathrm{CDCl}_{3}\right)$ spectrum of $\mathbf{2 d}$

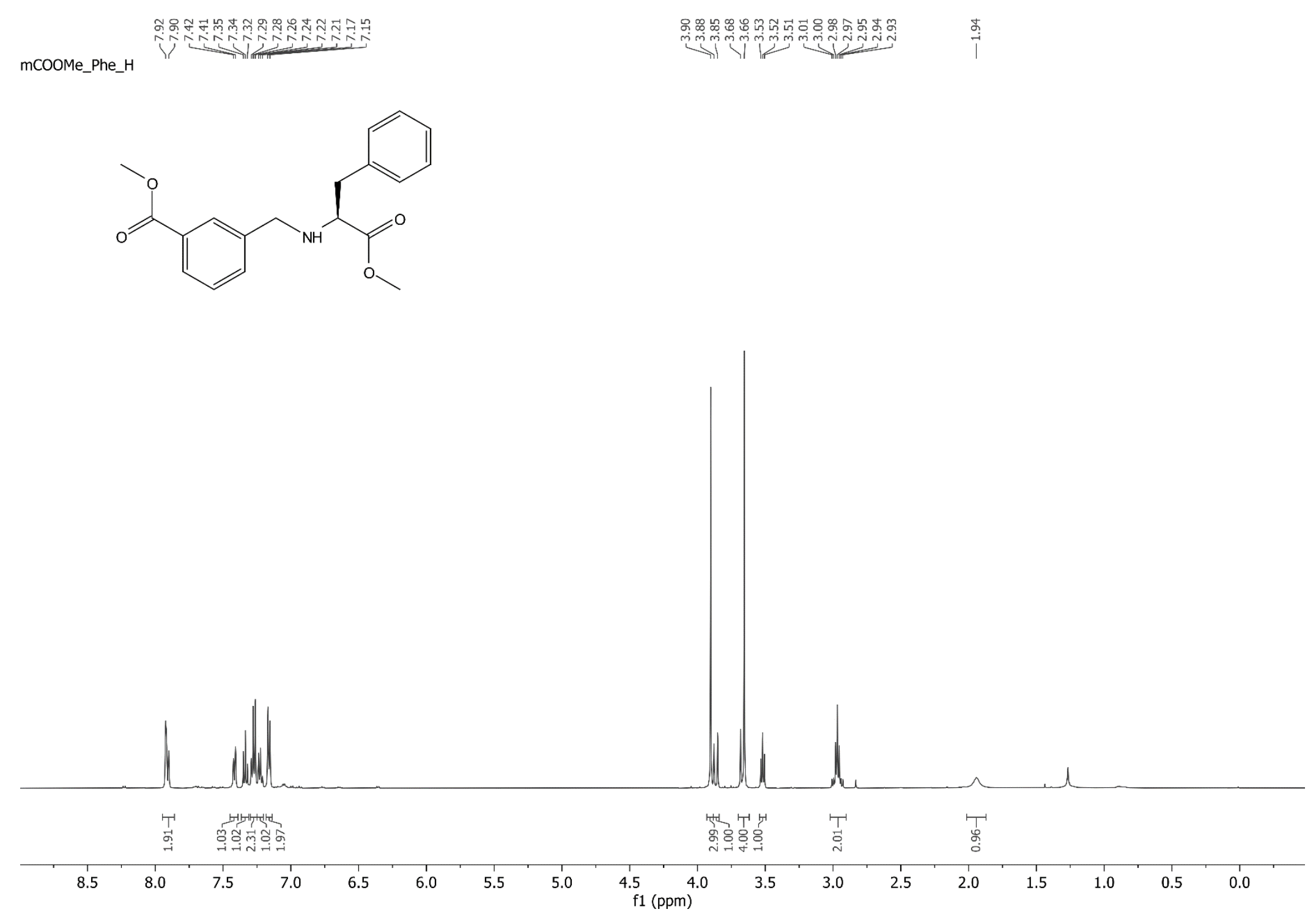


${ }^{13} \mathrm{C} \mathrm{NMR} \mathrm{(126} \mathrm{MHz,} \mathrm{CDCl}_{3}$ ) spectrum of $\mathbf{2 d}$

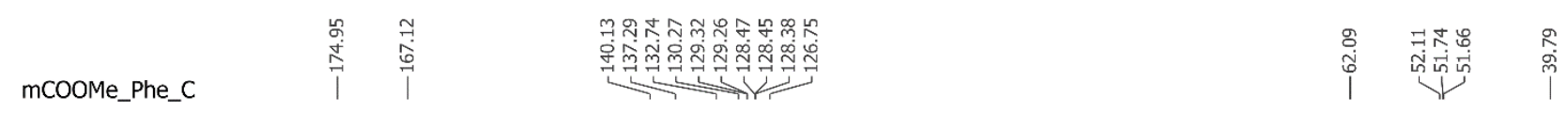
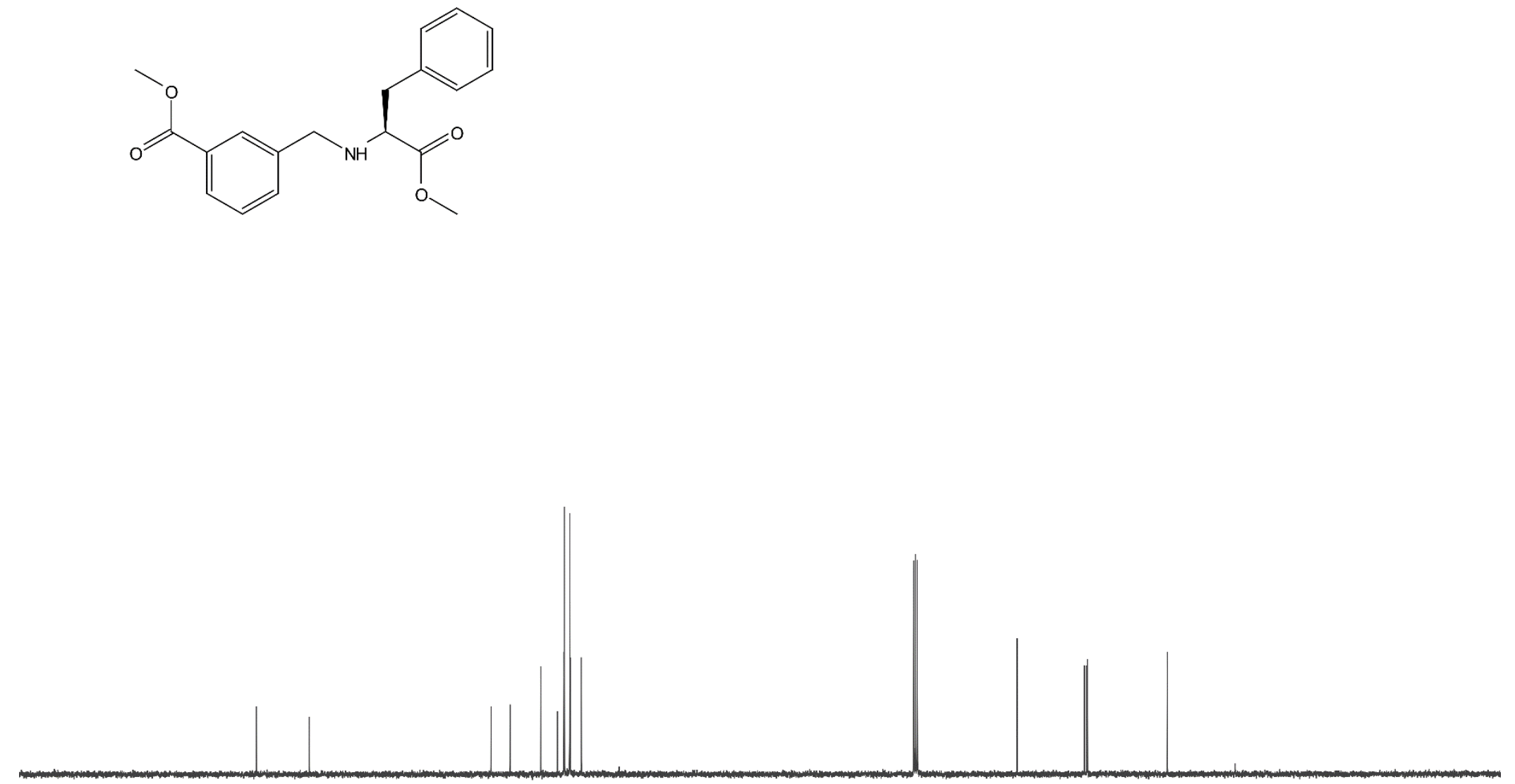

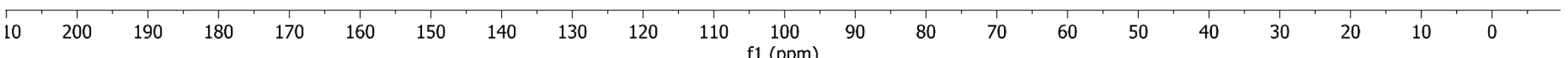


${ }^{1} \mathrm{H} \mathrm{NMR}\left(600 \mathrm{MHz}, \mathrm{CDCl}_{3}\right)$ spectrum of $\mathbf{2 e}$

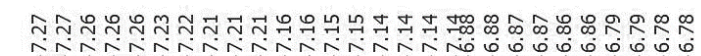
oOMe_Phe_H

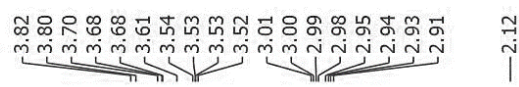

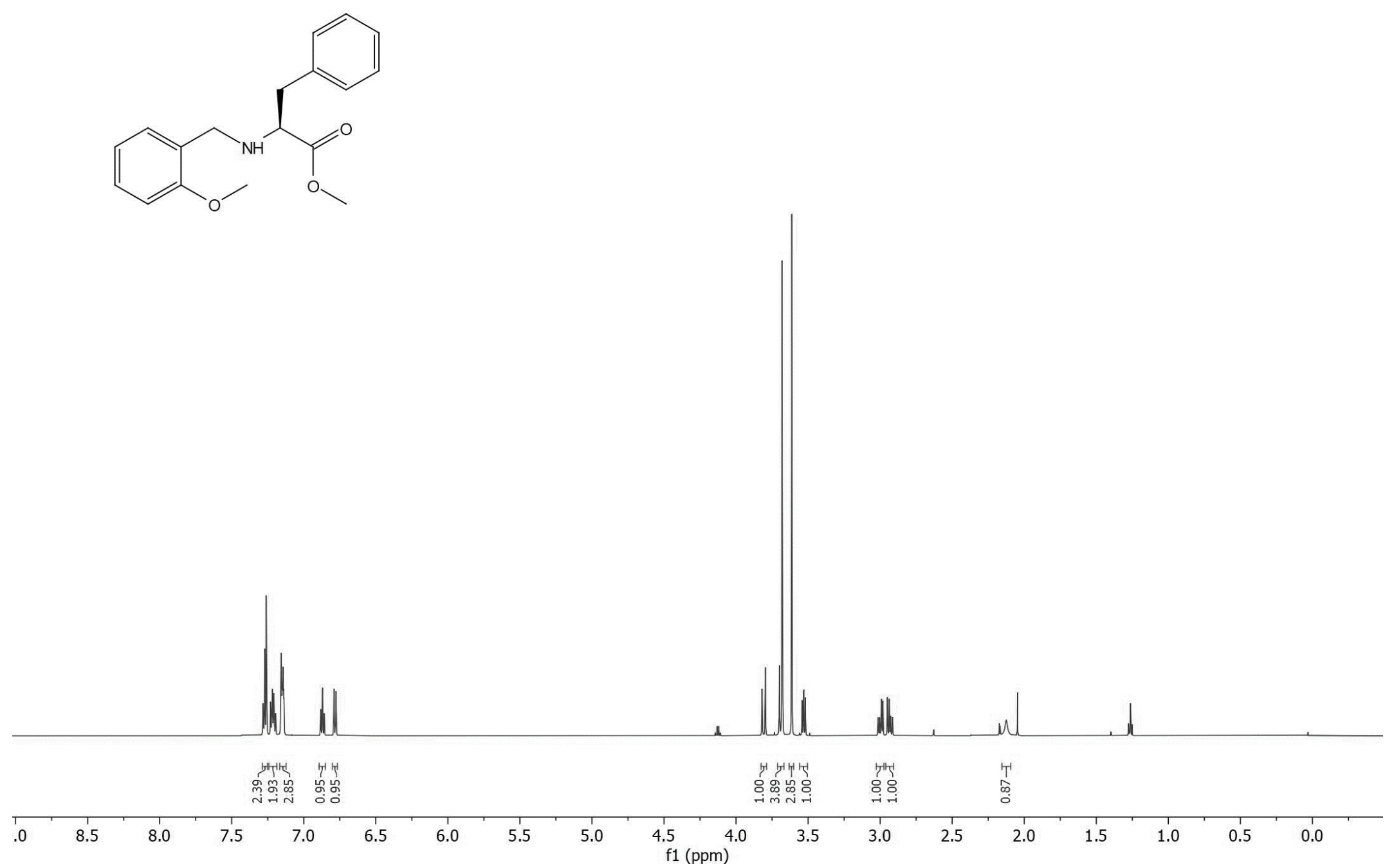


${ }^{13} \mathrm{C}$ NMR $\left(151 \mathrm{MHz}, \mathrm{CDCl}_{3}\right)$ spectrum of $\mathbf{2 e}$

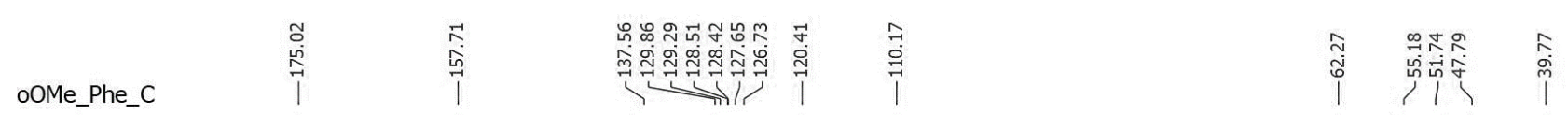<smiles>COC(=O)C(Cc1ccccc1)NCc1ccccc1OC</smiles>

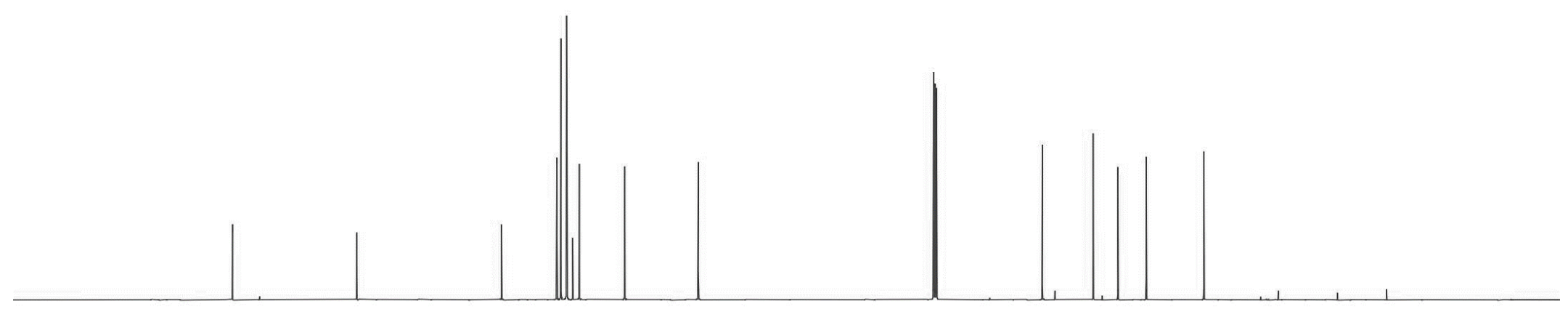

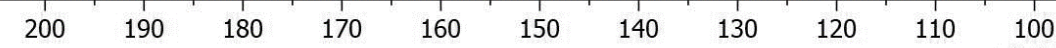

100
$\mathrm{f} 1(\mathrm{ppm})$ 
${ }^{1} \mathrm{H} \mathrm{NMR}\left(600 \mathrm{MHz}, \mathrm{CDCl}_{3}\right)$ spectrum of $\mathbf{2 f}$

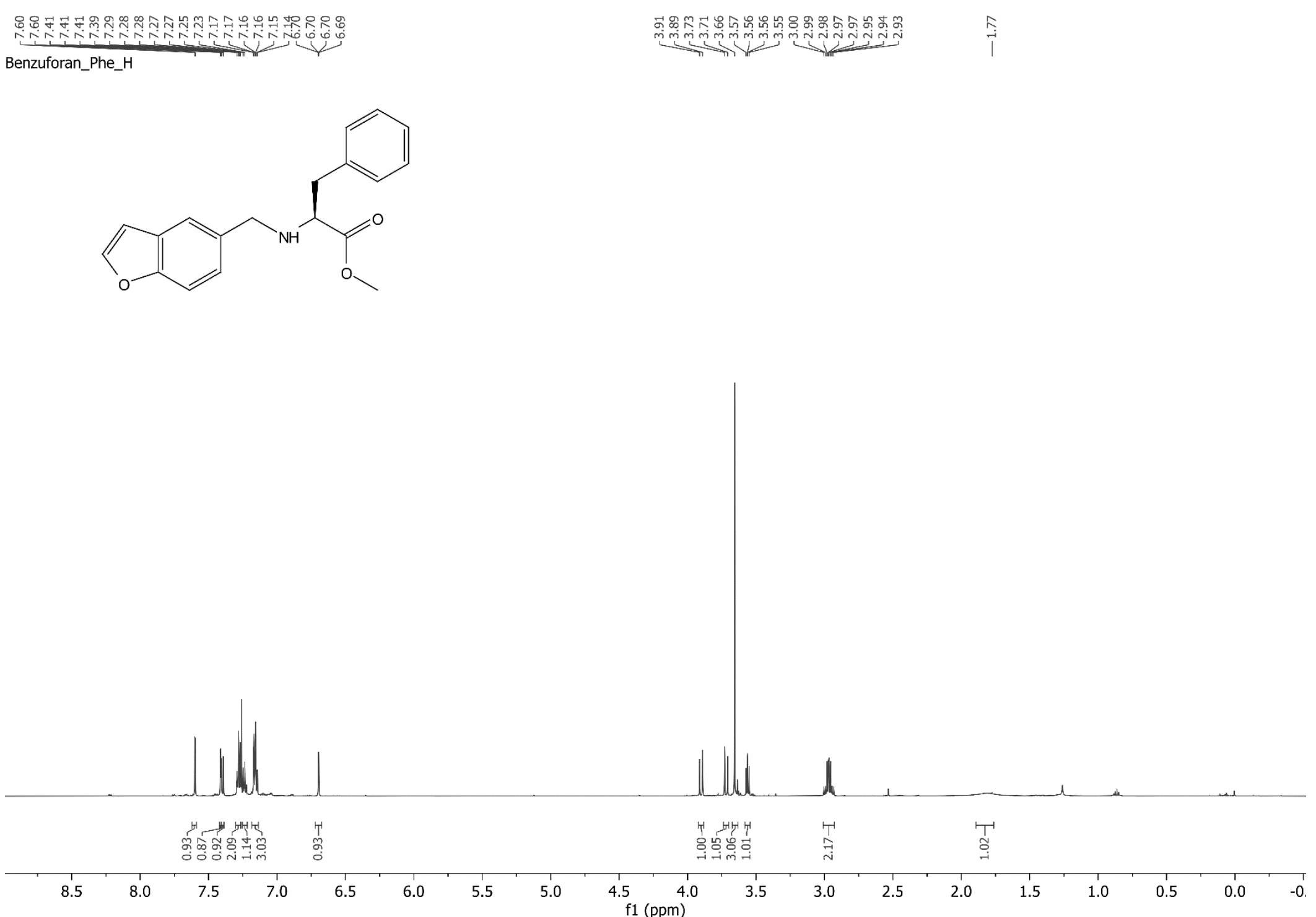


${ }^{13} \mathrm{C} \mathrm{NMR}\left(151 \mathrm{MHz}, \mathrm{CDCl}_{3}\right)$ spectrum of $\mathbf{2 f}$

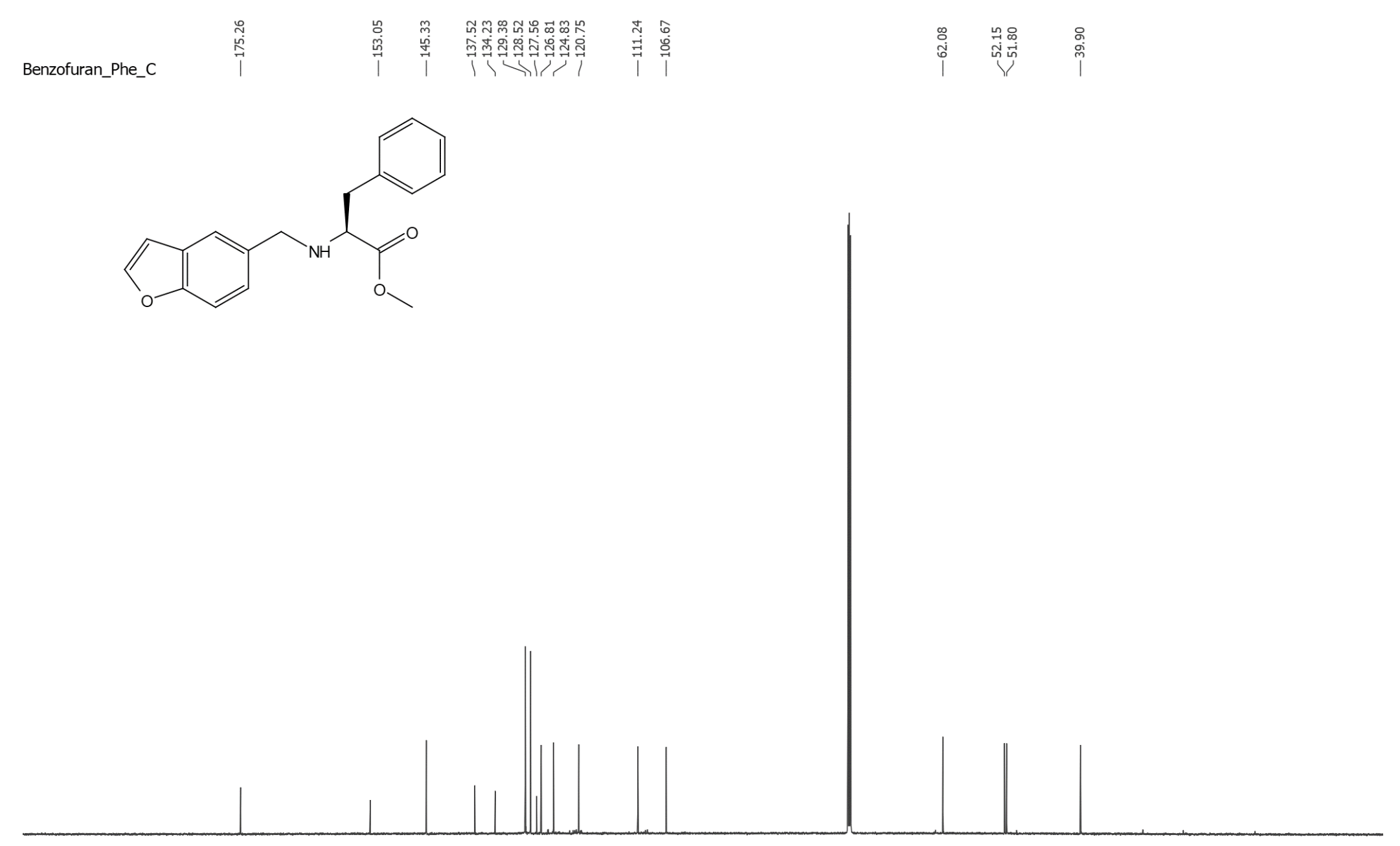

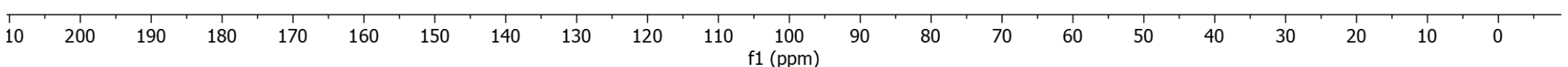


${ }^{1} \mathrm{H}$ NMR $\left(600 \mathrm{MHz}, \mathrm{CDCl}_{3}\right)$ spectrum of $\mathbf{2 g}$

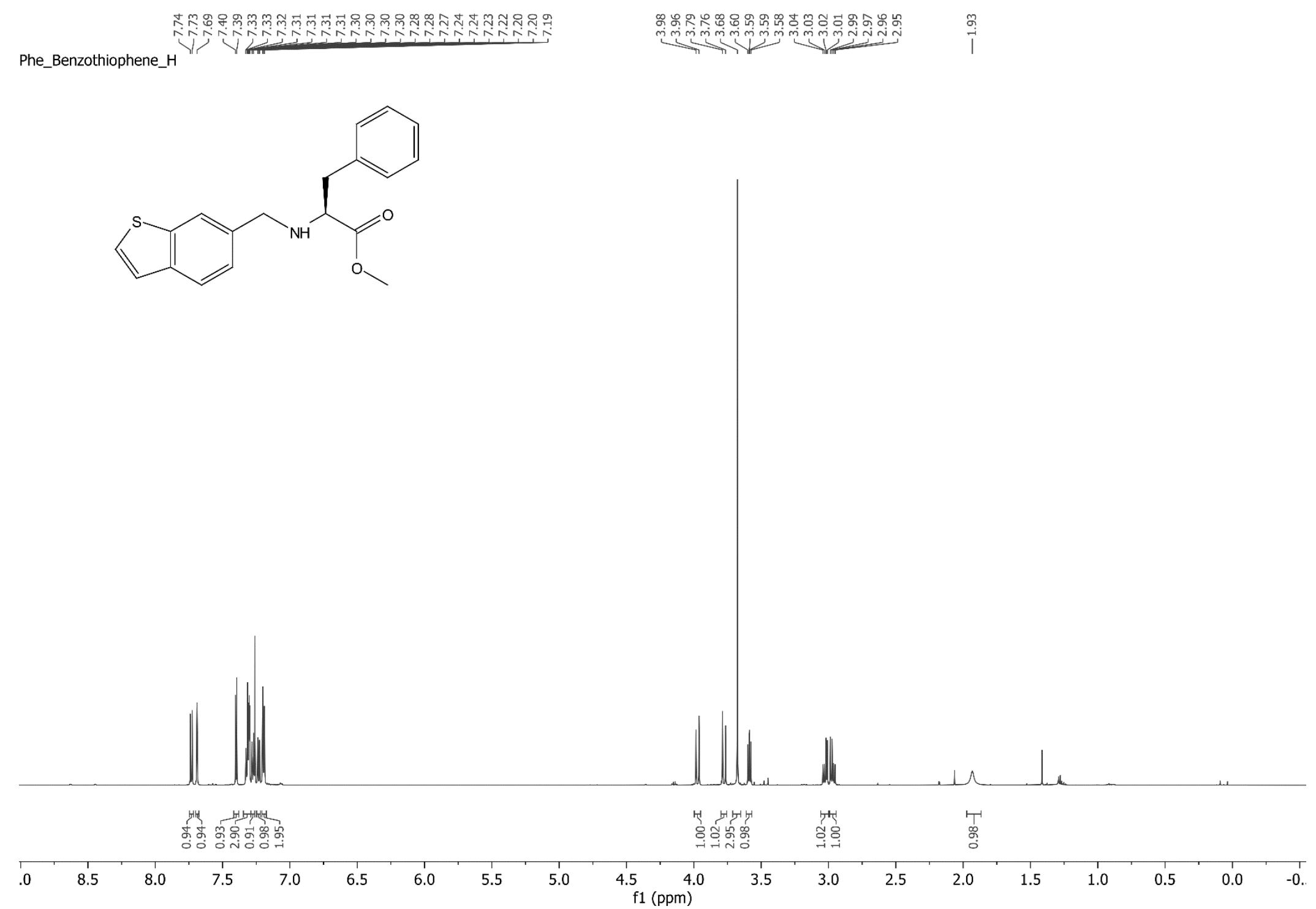


${ }^{13} \mathrm{C} \mathrm{NMR} \mathrm{(151} \mathrm{MHz,} \mathrm{CDCl}_{3}$ ) spectrum of $\mathbf{2 g}$

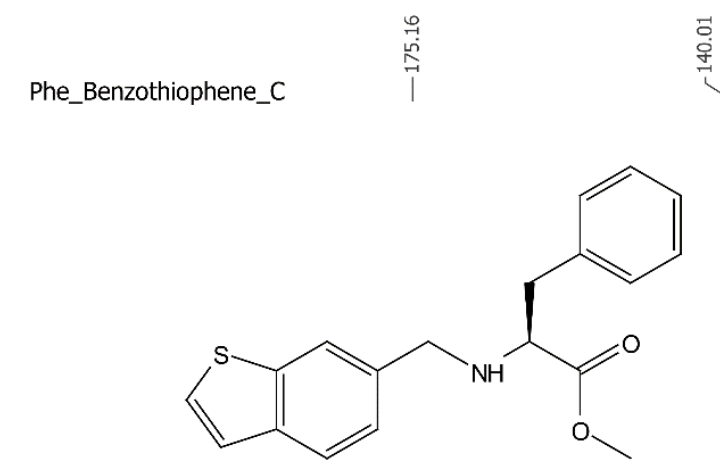

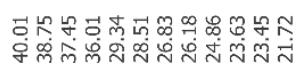

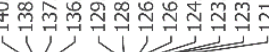

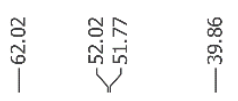

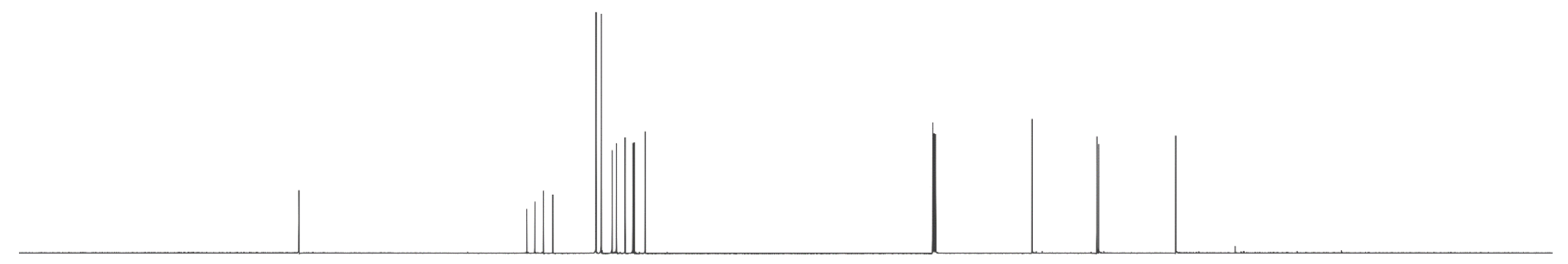


${ }^{1} \mathrm{H}$ NMR $\left(600 \mathrm{MHz}, \mathrm{CDCl}_{3}\right)$ spectrum of $\mathbf{2 h}$

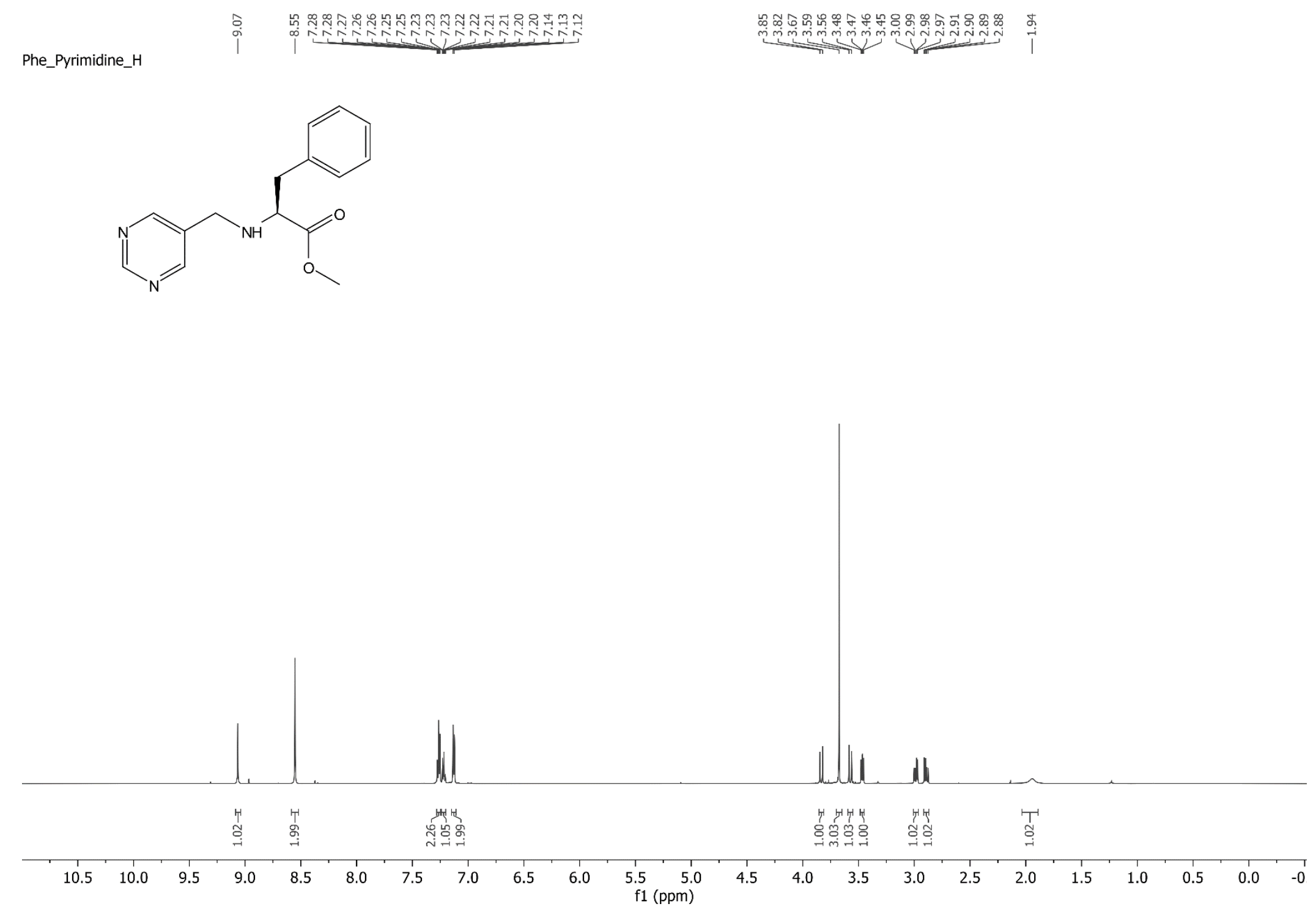


${ }^{13} \mathrm{C}$ NMR (151 MHz, $\mathrm{CDCl}_{3}$ ) spectrum of $\mathbf{2 h}$
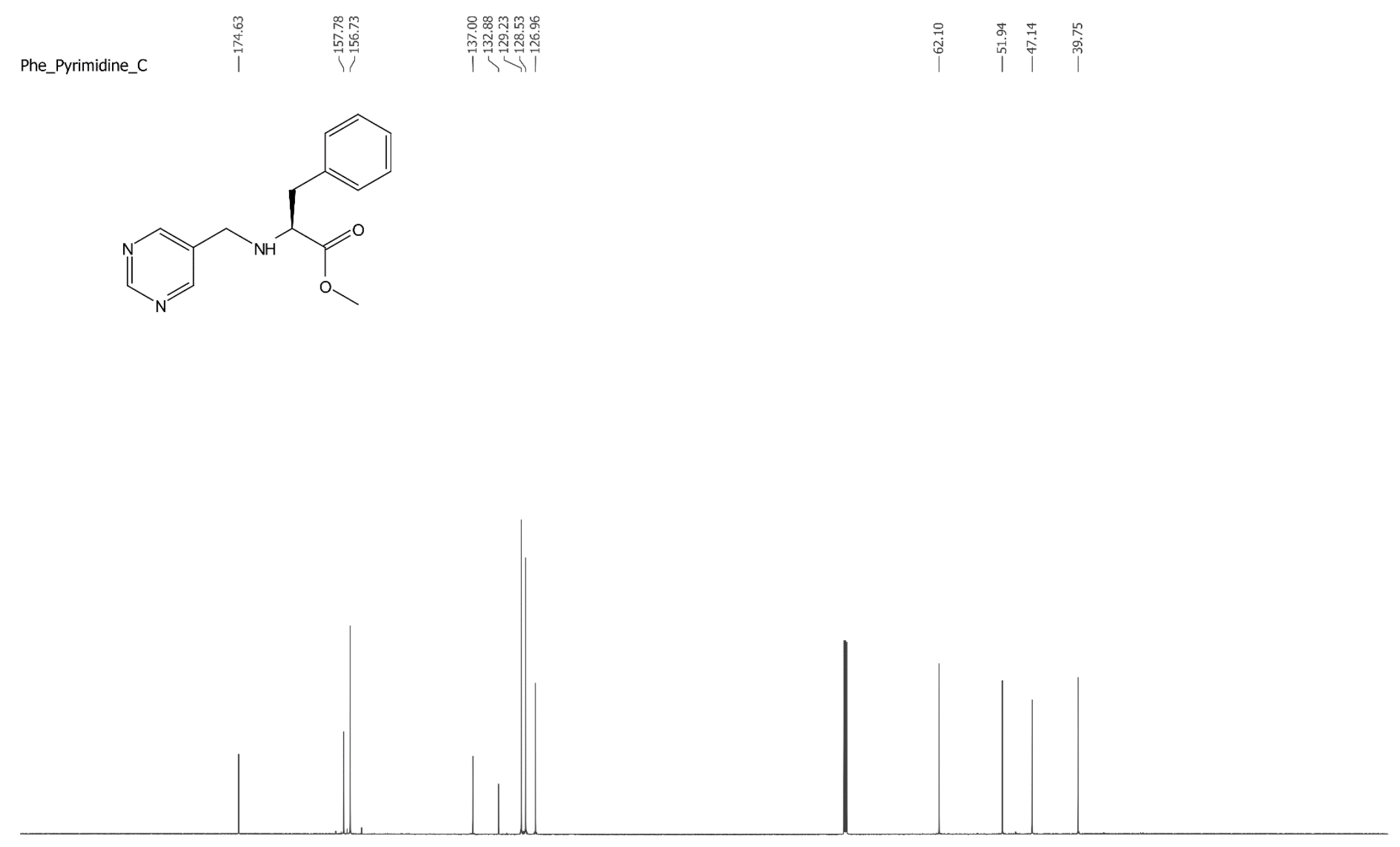

$\begin{array}{lllllllllllllllllllllllllll} & 1 & 1 & 1 & 180 & 170 & 160 & 150 & 140 & 130 & 120 & 110 & 100 & 90 & 80 & 70 & 60 & 50 & 40 & 30 & 20 & 10 & 0 & -1\end{array}$ 
${ }^{1} \mathrm{H}$ NMR $\left(600 \mathrm{MHz}, \mathrm{CDCl}_{3}\right)$ spectrum of $\mathbf{2} \mathbf{i}$

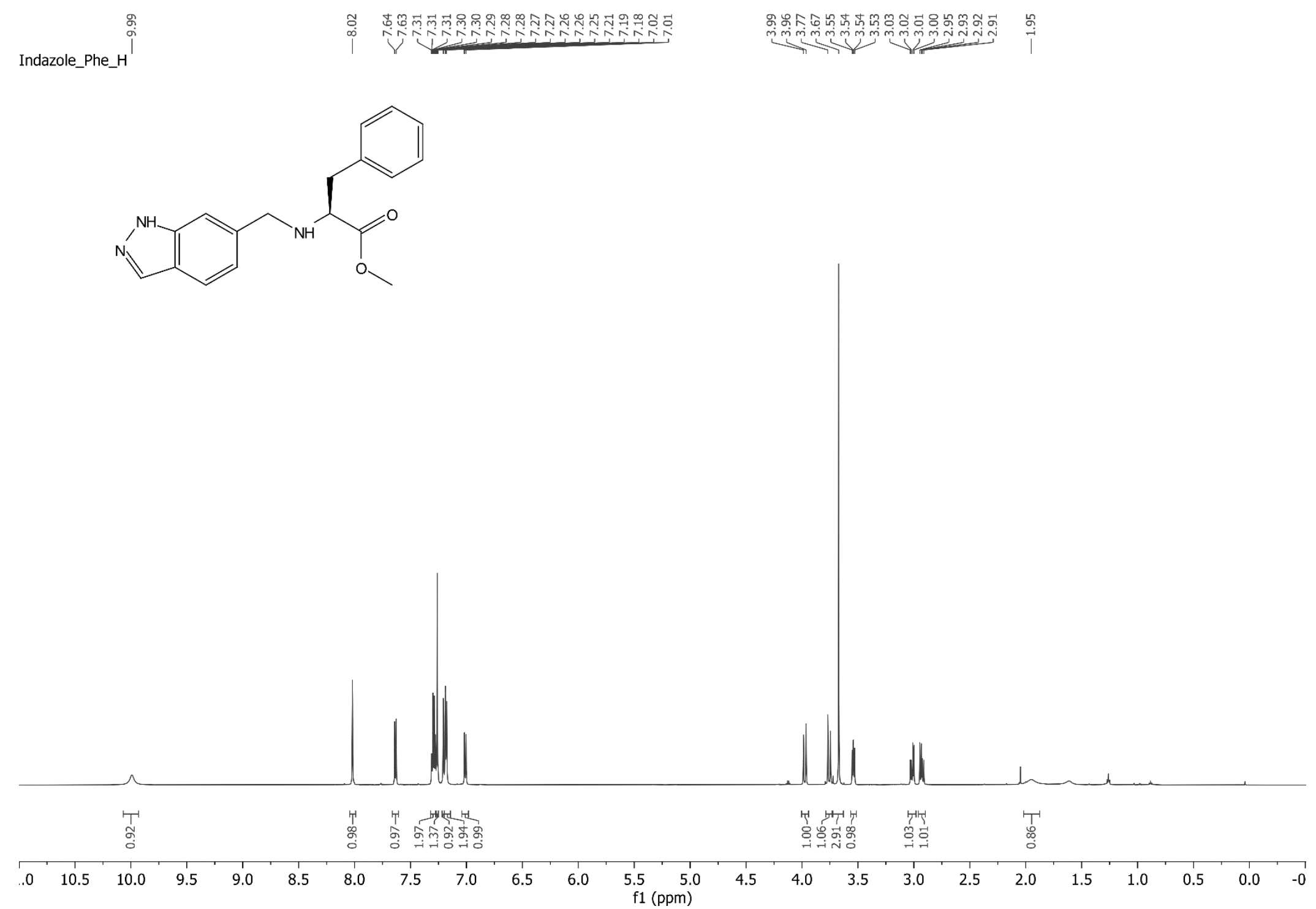


${ }^{13} \mathrm{C}$ NMR $\left(151 \mathrm{MHz}, \mathrm{CDCl}_{3}\right)$ spectrum of $\mathbf{2 i}$

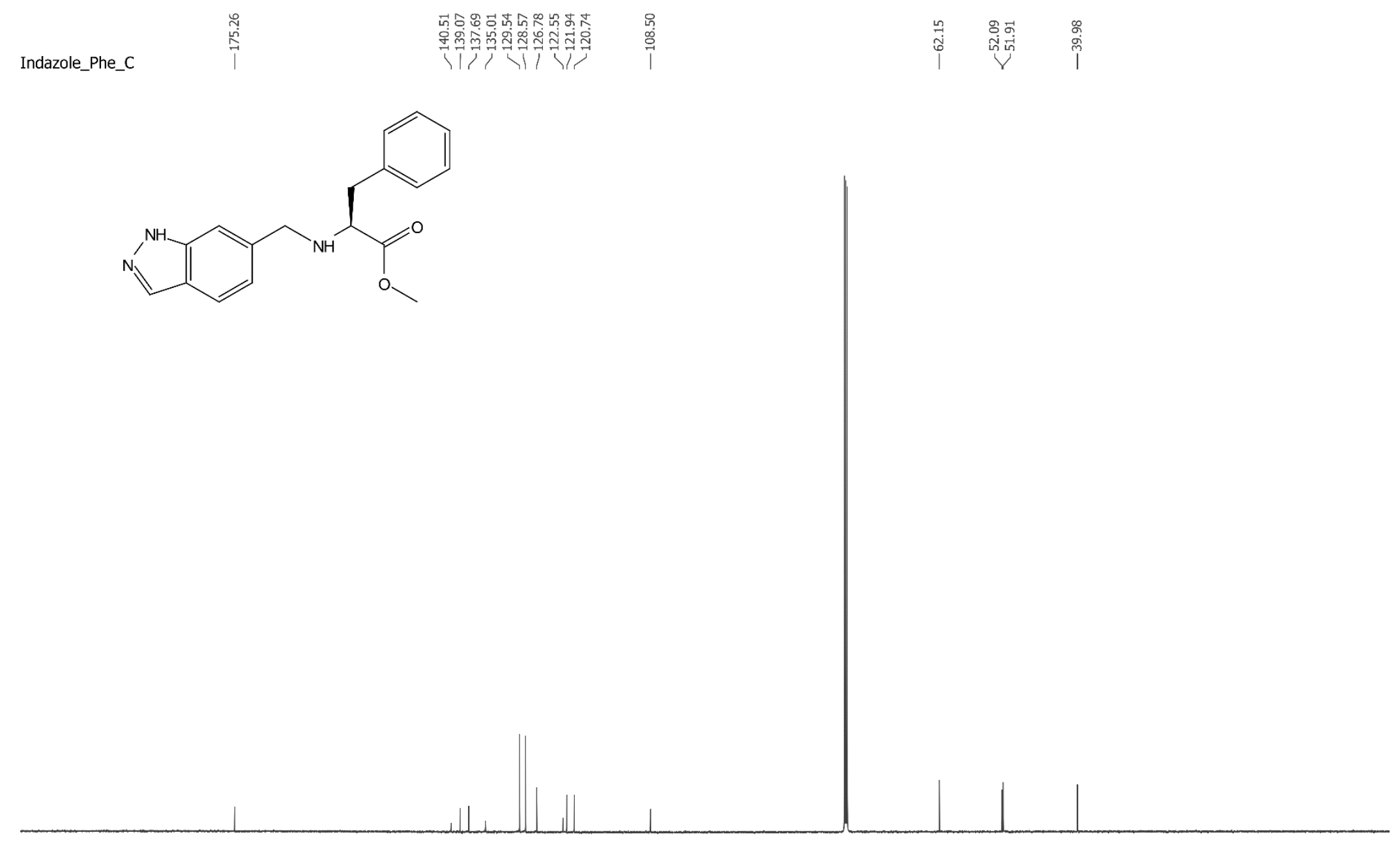

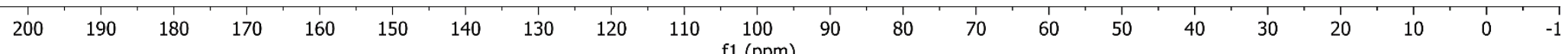


${ }^{1} \mathrm{H}$ NMR $\left(600 \mathrm{MHz}, \mathrm{CDCl}_{3}\right)$ spectrum of $\mathbf{2 j}$

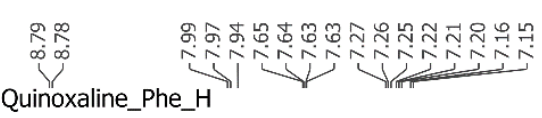

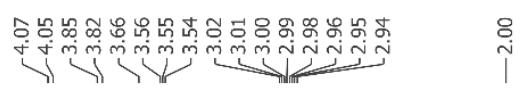
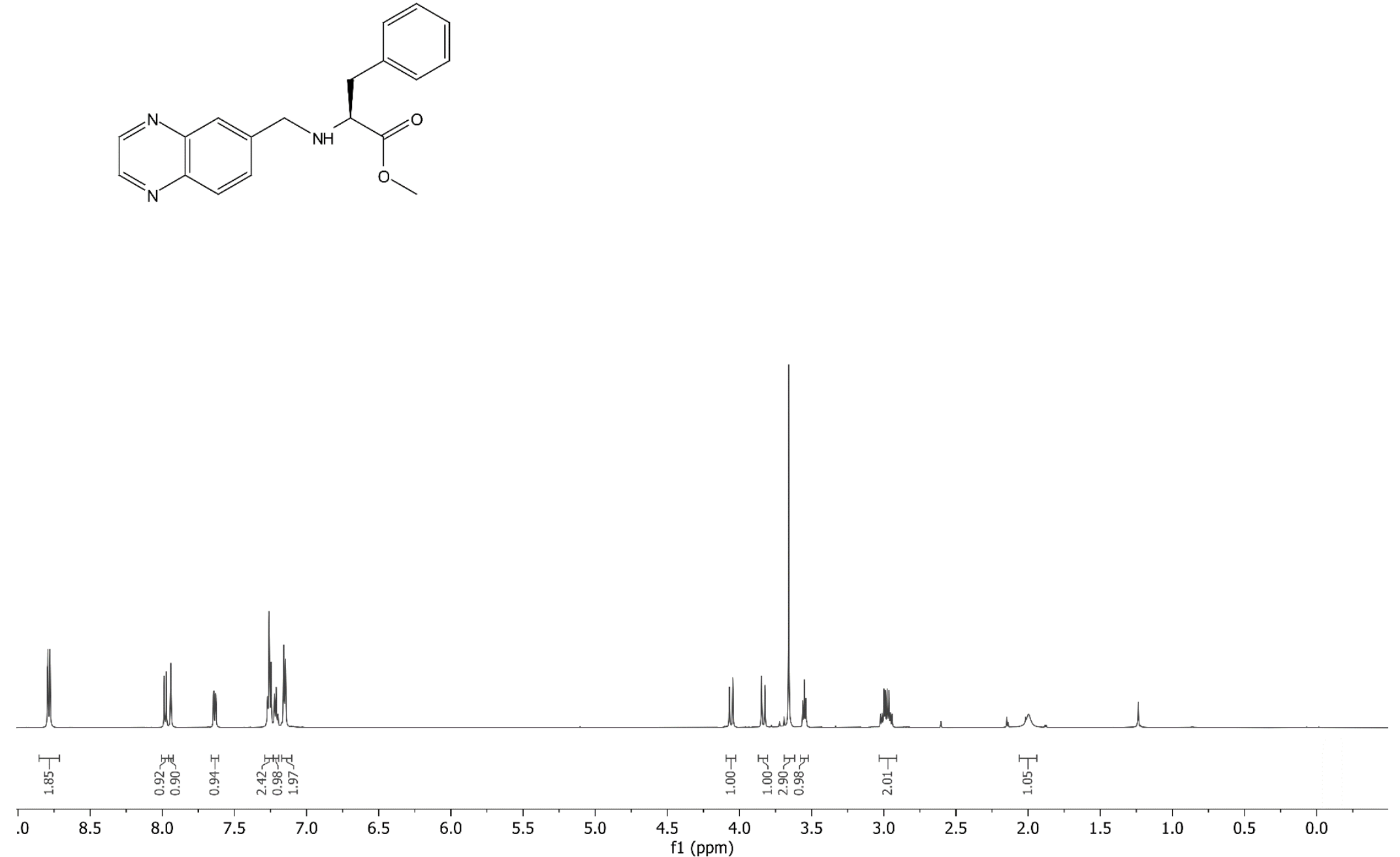
${ }^{13} \mathrm{C} \mathrm{NMR}\left(151 \mathrm{MHz}, \mathrm{CDCl}_{3}\right)$ spectrum of $\mathbf{2 j}$

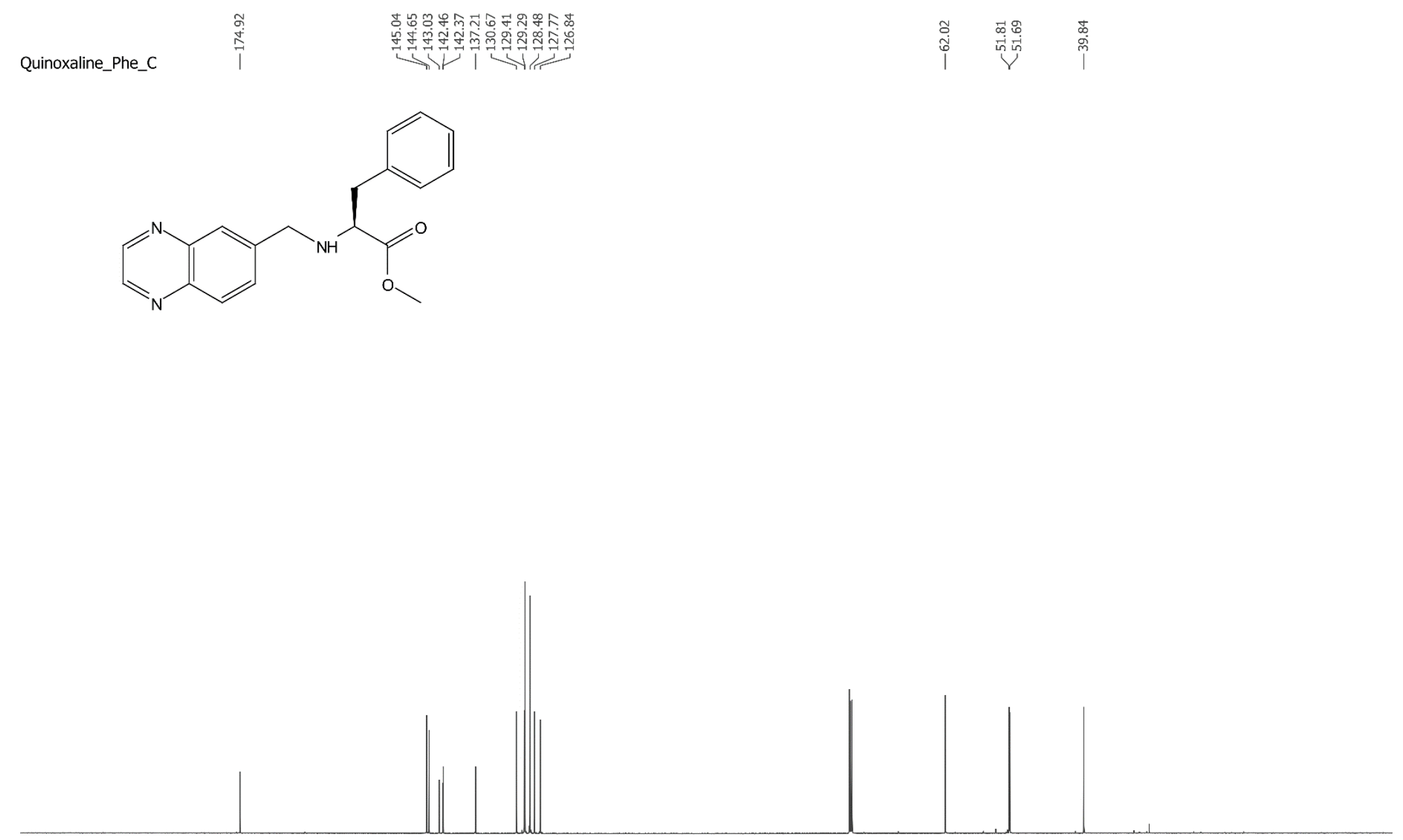

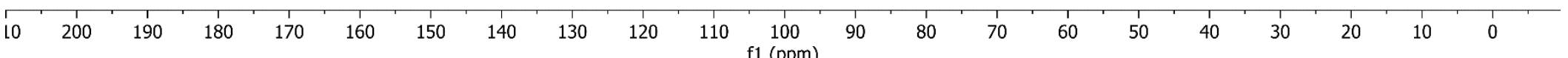


${ }^{1} \mathrm{H}$ NMR (600 MHz, $\mathrm{CDCl}_{3}$ ) spectrum of $\mathbf{2 k}$

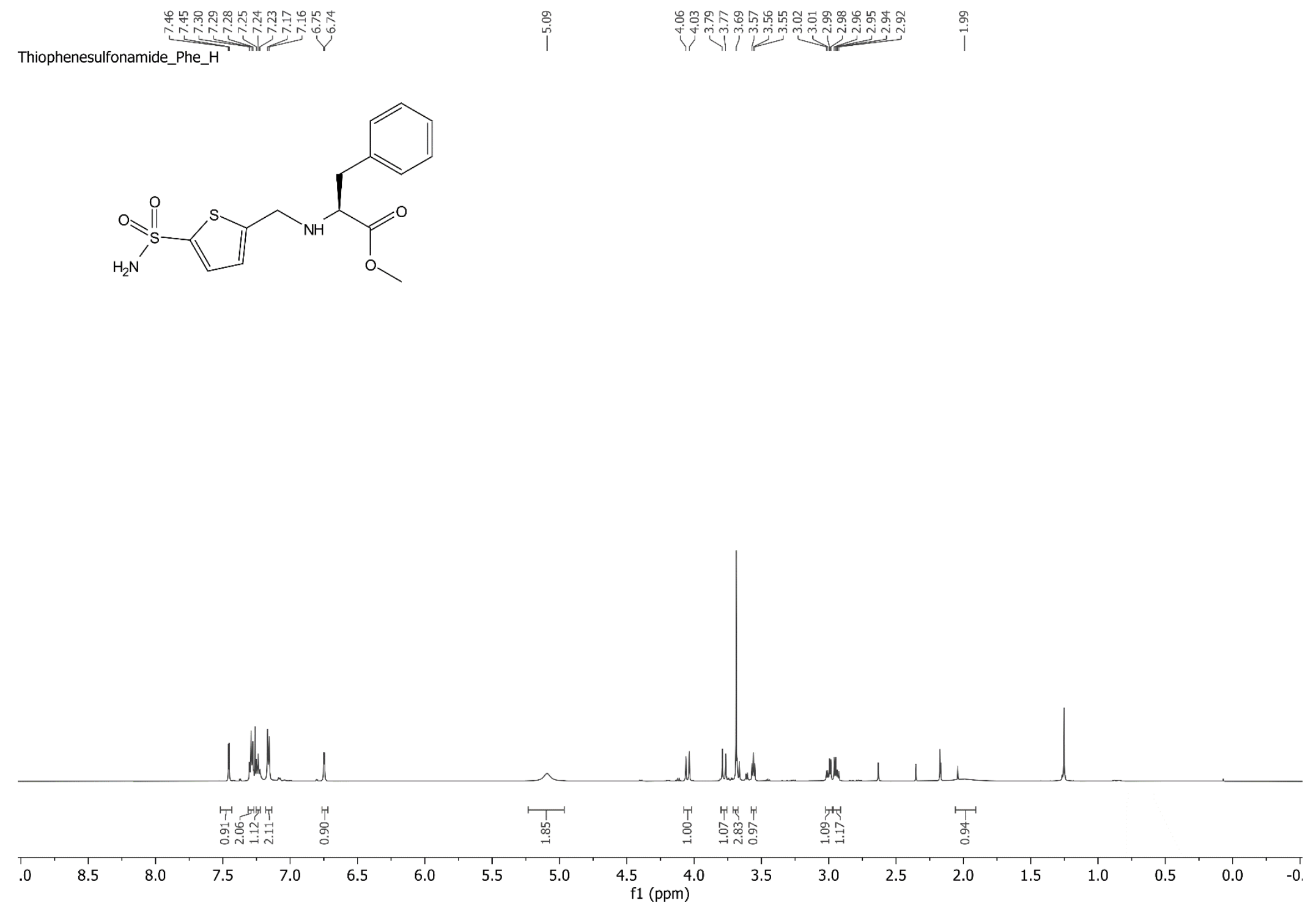


${ }^{13} \mathrm{C}$ NMR (151 MHz, $\mathrm{CDCl}_{3}$ ) spectrum of $\mathbf{2 k}$
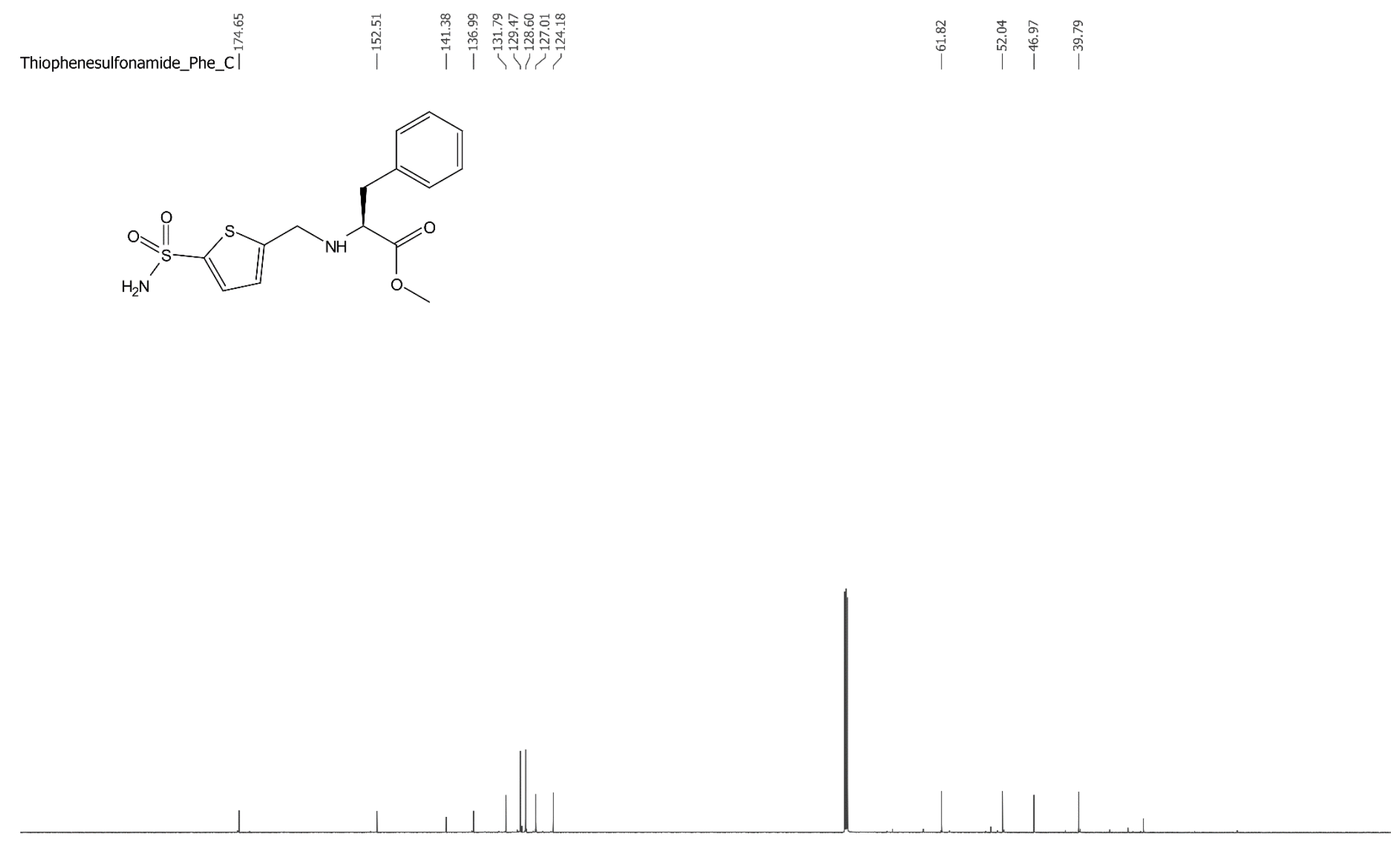

$\begin{array}{lllllllllllllllllllllll} & 190 & 190 & 180 & 170 & 160 & 150 & 140 & 130 & 120 & 110 & 100 & 90 & 80 & 70 & 60 & 50 & 40 & 30 & 20 & 10 & 0 & -1\end{array}$ 
${ }^{1} \mathrm{H}$ NMR $\left(600 \mathrm{MHz}, \mathrm{CDCl}_{3}\right)$ spectrum of $\mathbf{2 l}$

\section{क}

PiperazinePyrimidine_Phe_H

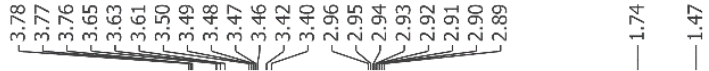<smiles>COC(=O)[C@H](Cc1ccccc1)NCc1cnc(N2CCN(C(=O)OC(C)(C)C)CC2)nc1</smiles>

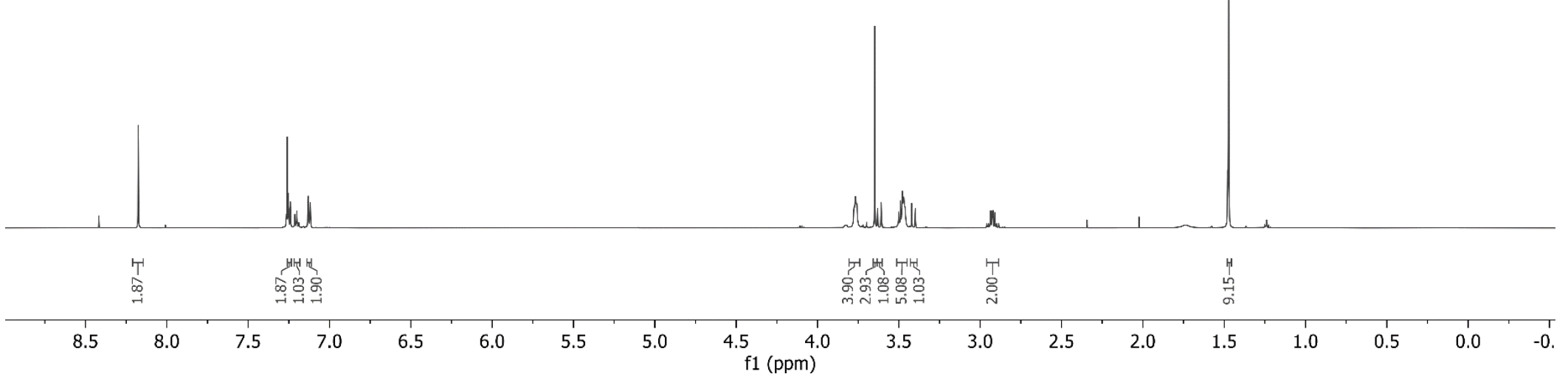


${ }^{13} \mathrm{C}$ NMR $\left(151 \mathrm{MHz}, \mathrm{CDCl}_{3}\right)$ spectrum of $\mathbf{2 l}$

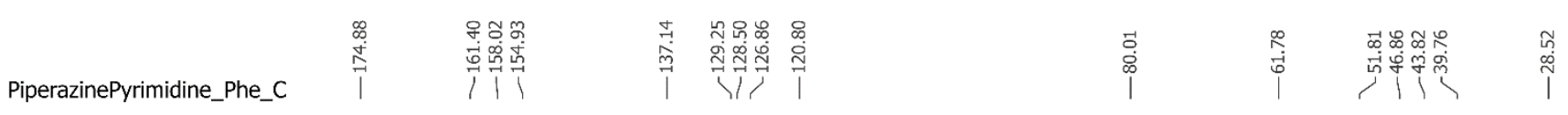
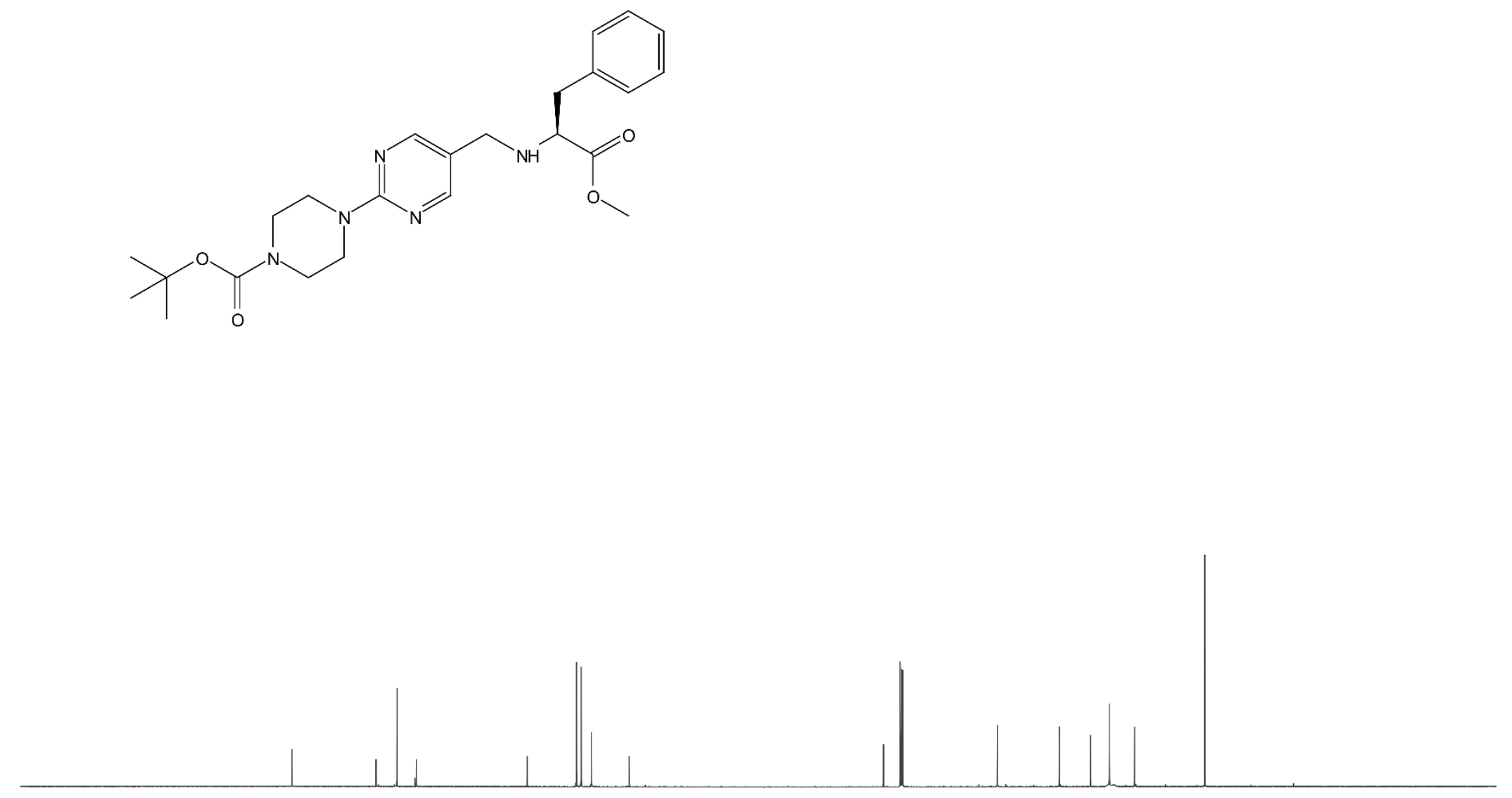
${ }^{1} \mathrm{H} \mathrm{NMR}\left(600 \mathrm{MHz}, \mathrm{CDCl}_{3}\right)$ spectrum of $\mathbf{2 m}$

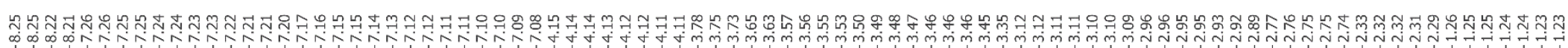
Loratadine_Phe_H
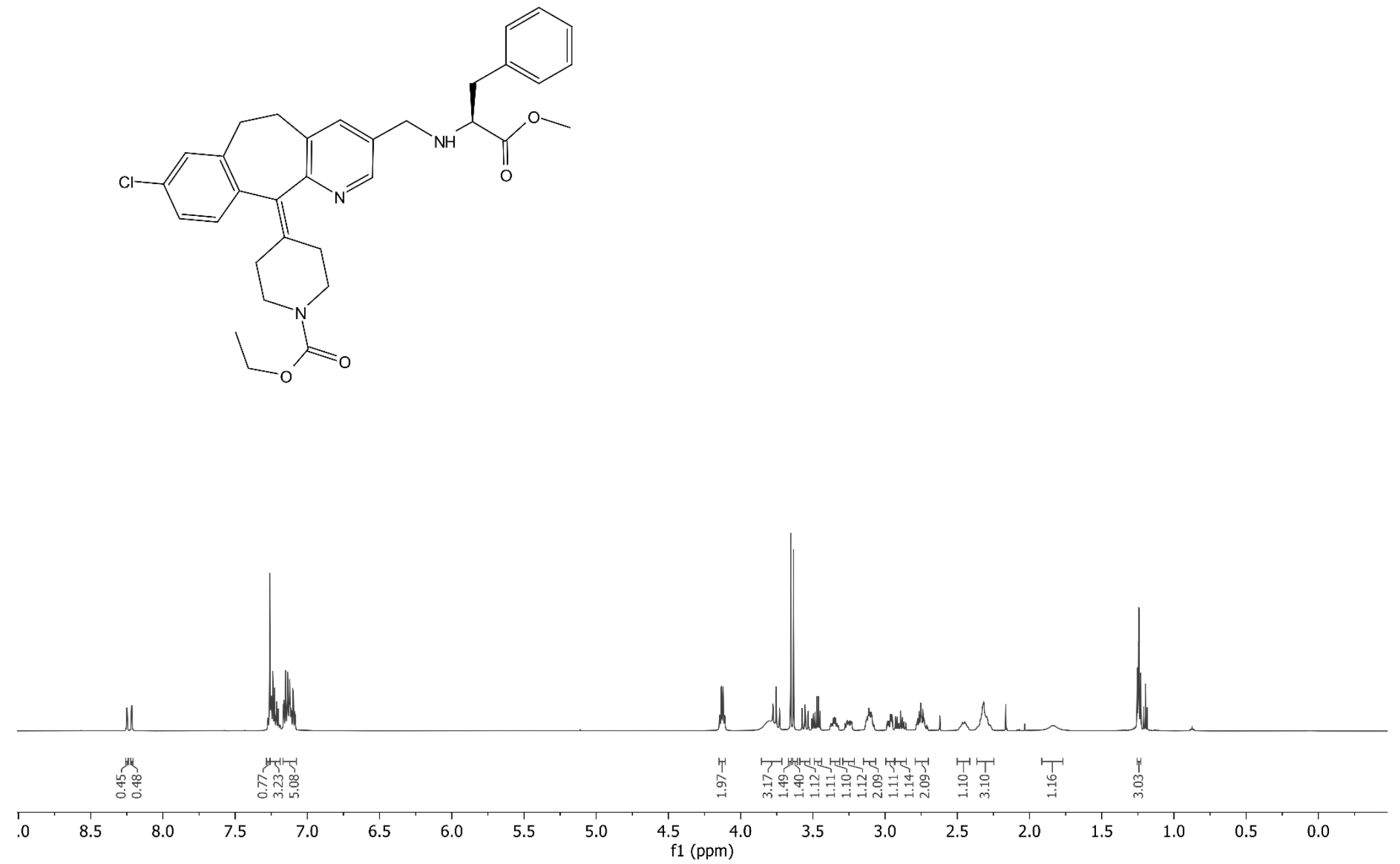
${ }^{13} \mathrm{C}$ NMR $\left(151 \mathrm{MHz}, \mathrm{CDCl}_{3}\right)$ spectrum of $\mathbf{2 m}$

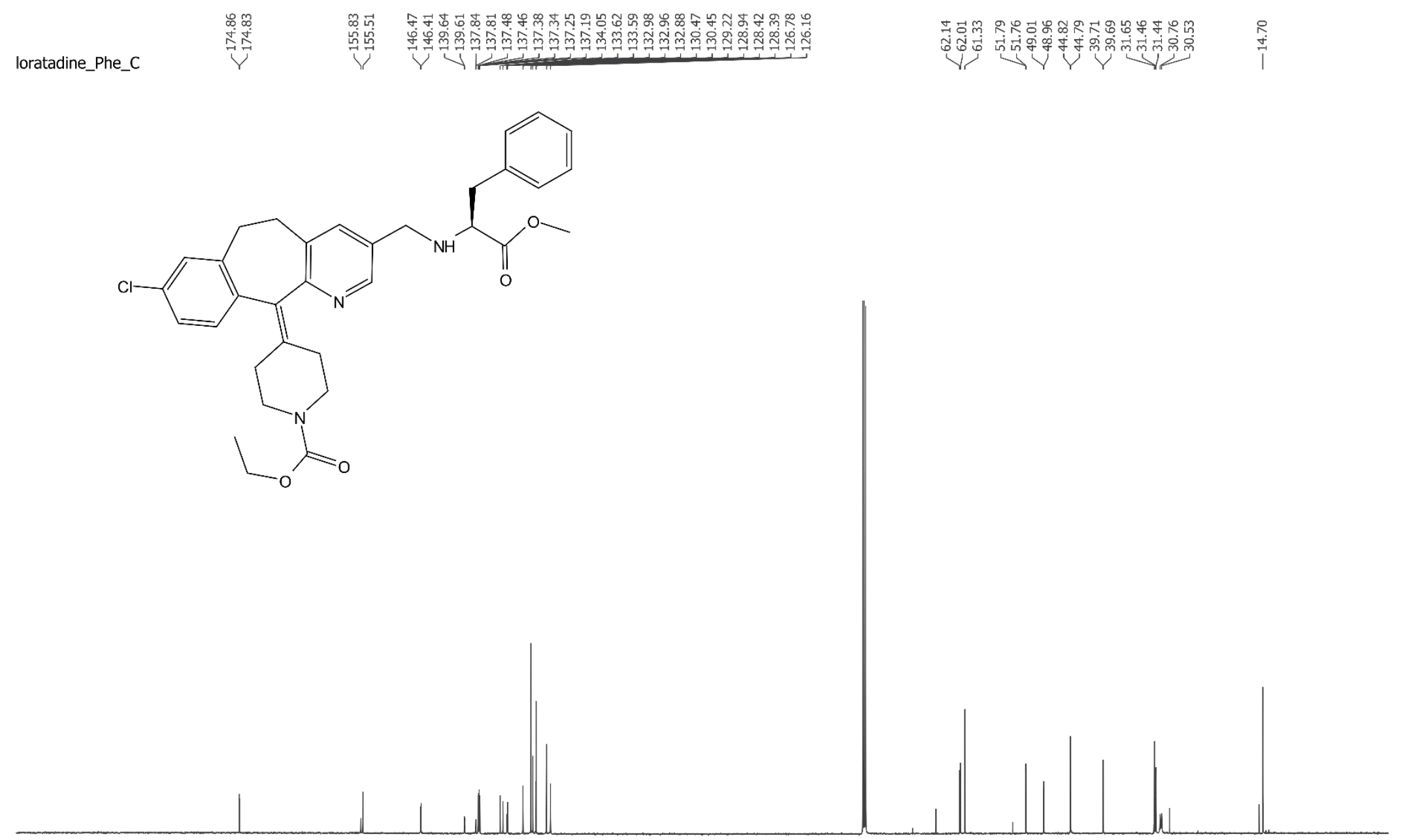

200
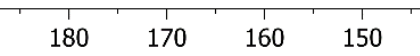

$140 \quad 130$

120

$110 \quad 100$

80

70

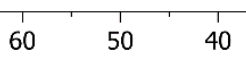

30

$2 0 \longdiv { 1 0 } \quad 0$ 
${ }^{1} \mathrm{H}$ NMR $\left(600 \mathrm{MHz}, \mathrm{CDCl}_{3}\right)$ spectrum of $\mathbf{2 n}$

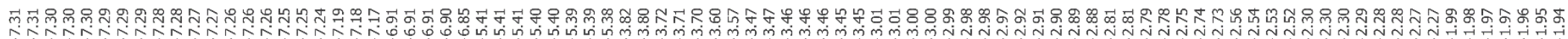
MerckX1_Phe_H
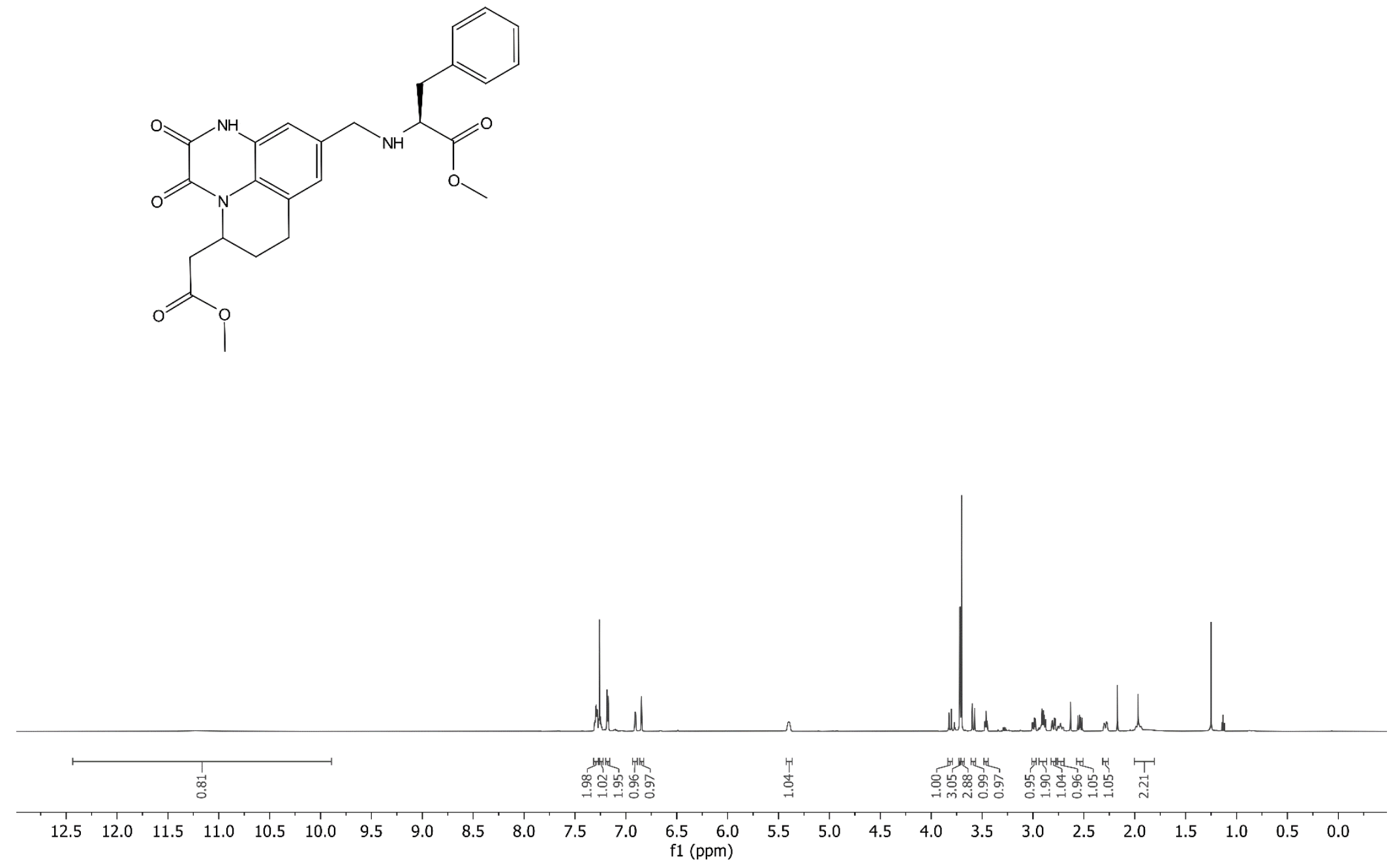
${ }^{13} \mathrm{C} \mathrm{NMR}\left(151 \mathrm{MHz}, \mathrm{CDCl}_{3}\right.$ ) spectrum of $\mathbf{2 n}$

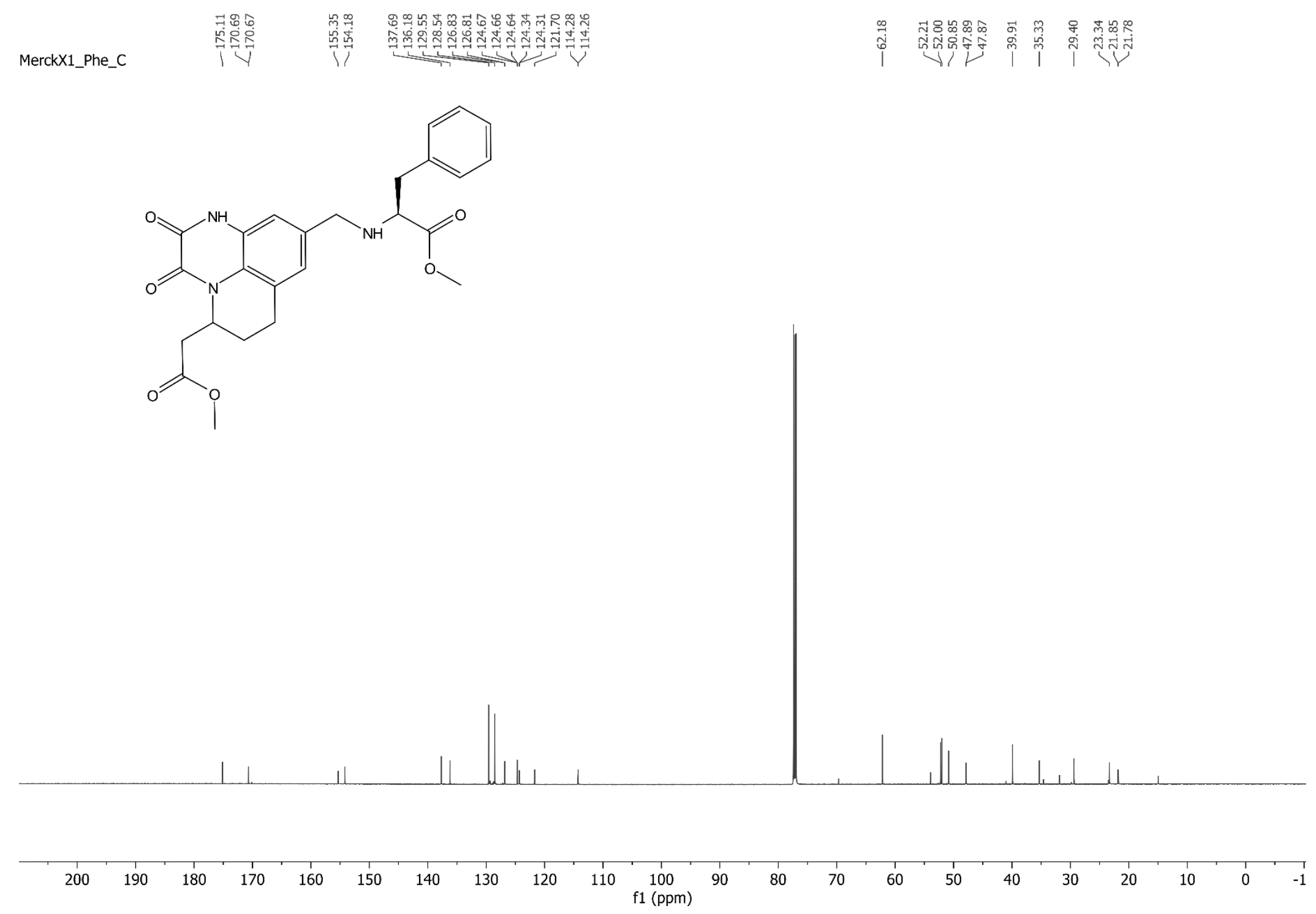


${ }^{1} \mathrm{H}$ NMR $\left(600 \mathrm{MHz}, \mathrm{CDCl}_{3}\right)$ spectrum of 20

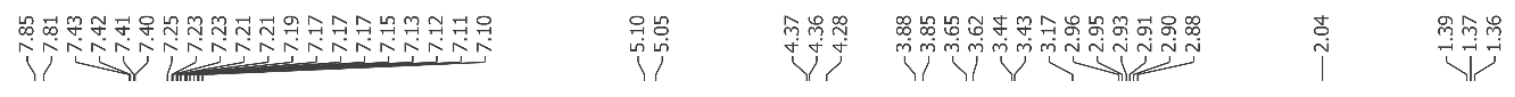

Flumazenil_Phe_H
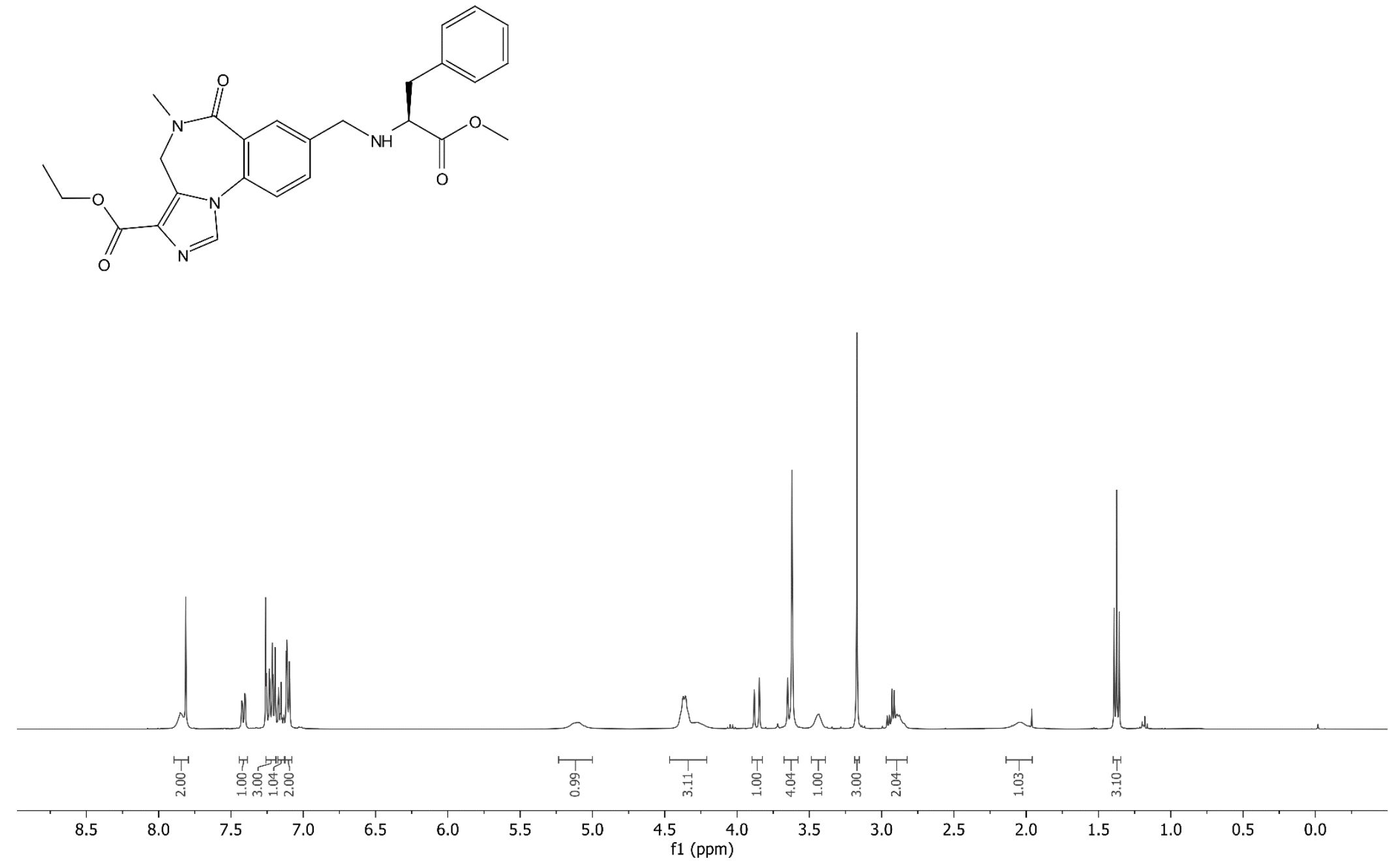
${ }^{13} \mathrm{C}$ NMR $\left(151 \mathrm{MHz}, \mathrm{CDCl}_{3}\right)$ spectrum of 20
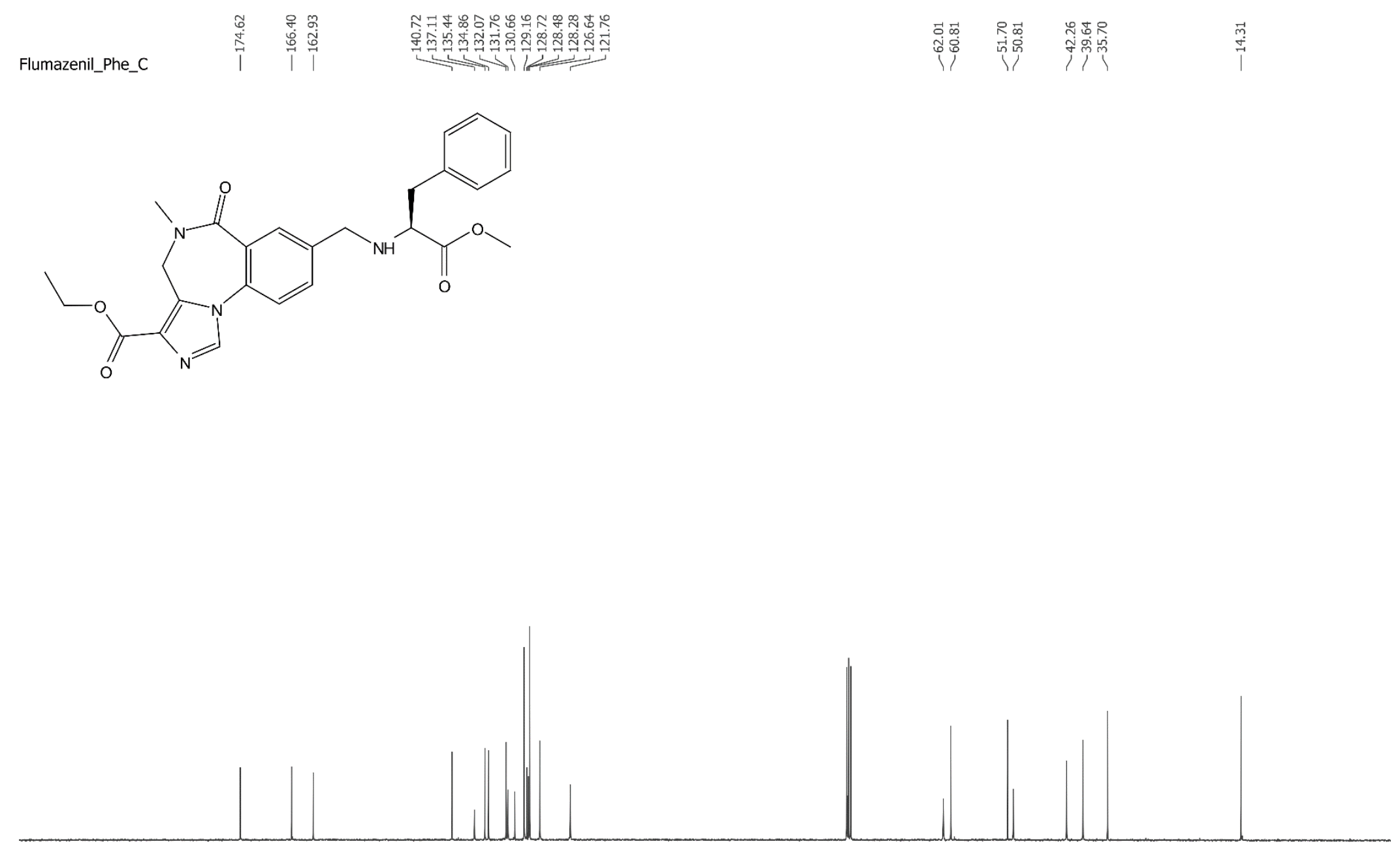

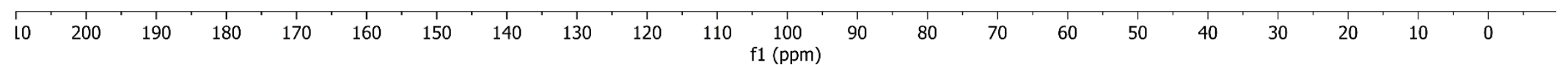


${ }^{1} \mathrm{H}$ NMR $\left(600 \mathrm{MHz}, \mathrm{CDCl}_{3}\right)$ spectrum of $\mathbf{2 p}$

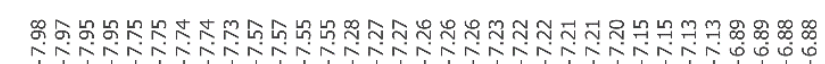

MerckX3_Phe_H

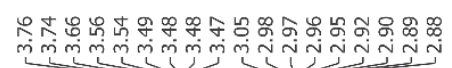
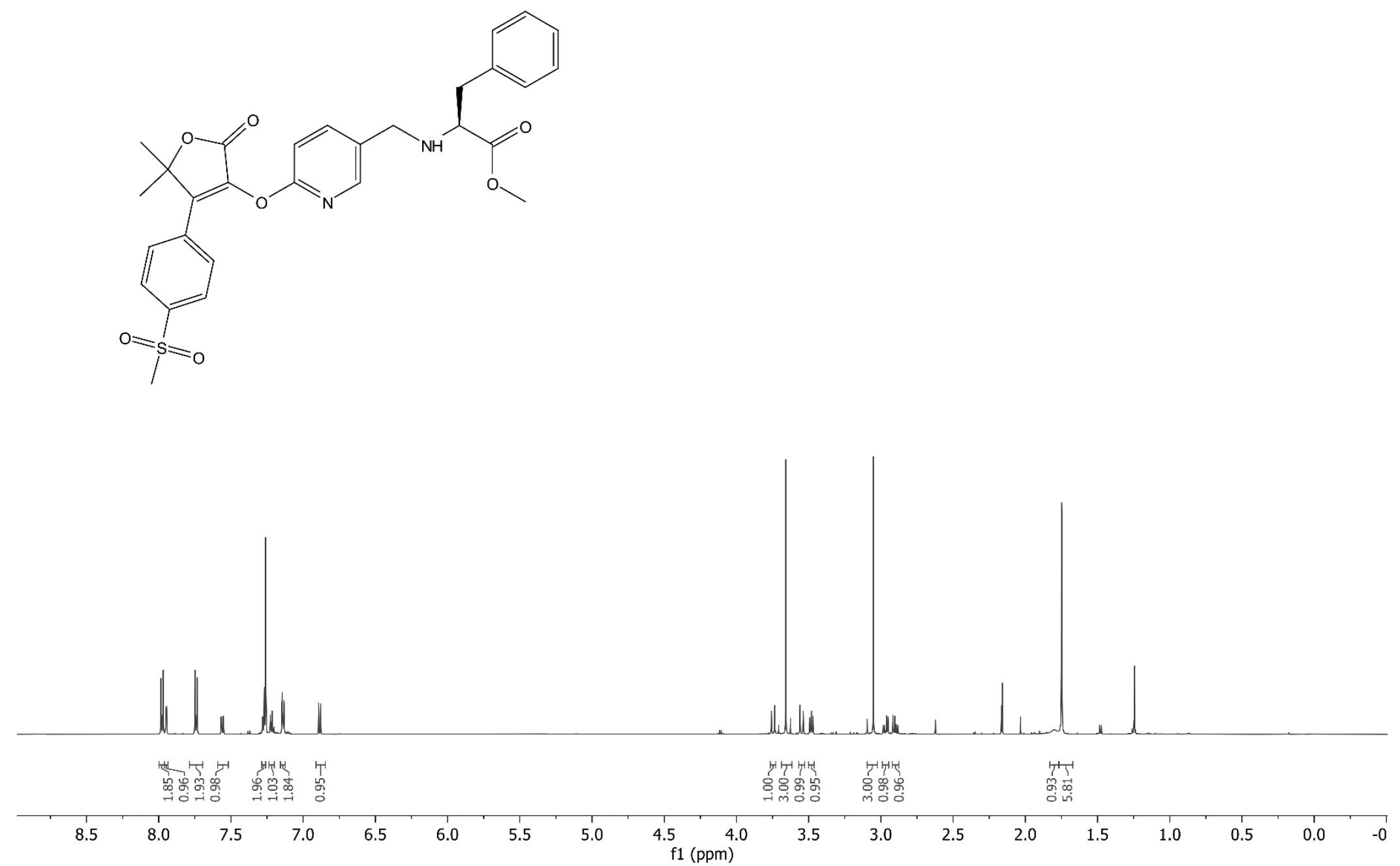
${ }^{13} \mathrm{C} \mathrm{NMR} \mathrm{(151} \mathrm{MHz,} \mathrm{CDCl}_{3}$ ) spectrum of $\mathbf{2 p}$
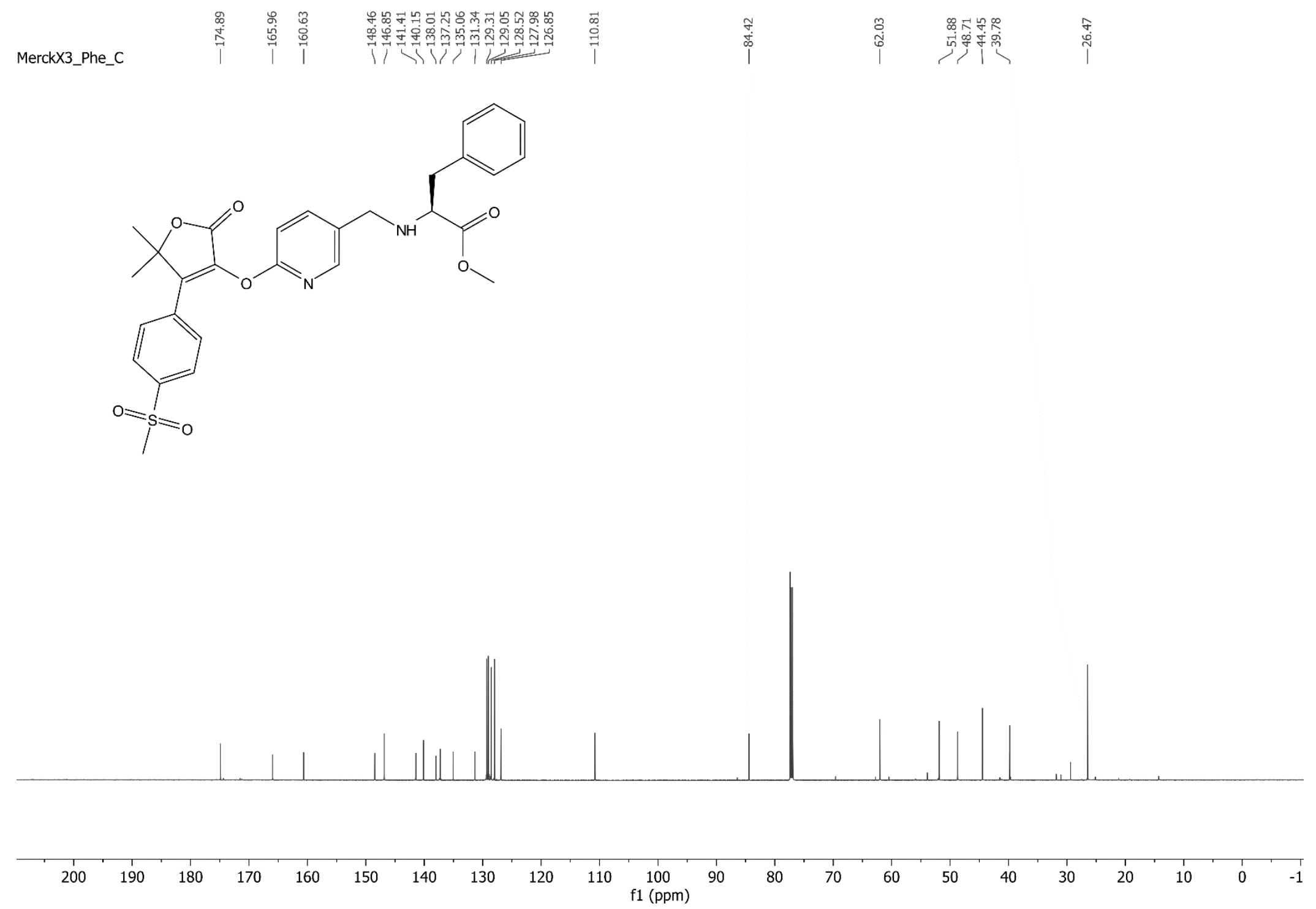
${ }^{1} \mathrm{H}$ NMR $\left(500 \mathrm{MHz}, \mathrm{CDCl}_{3}\right.$ ) spectrum of $\mathbf{2 q}$

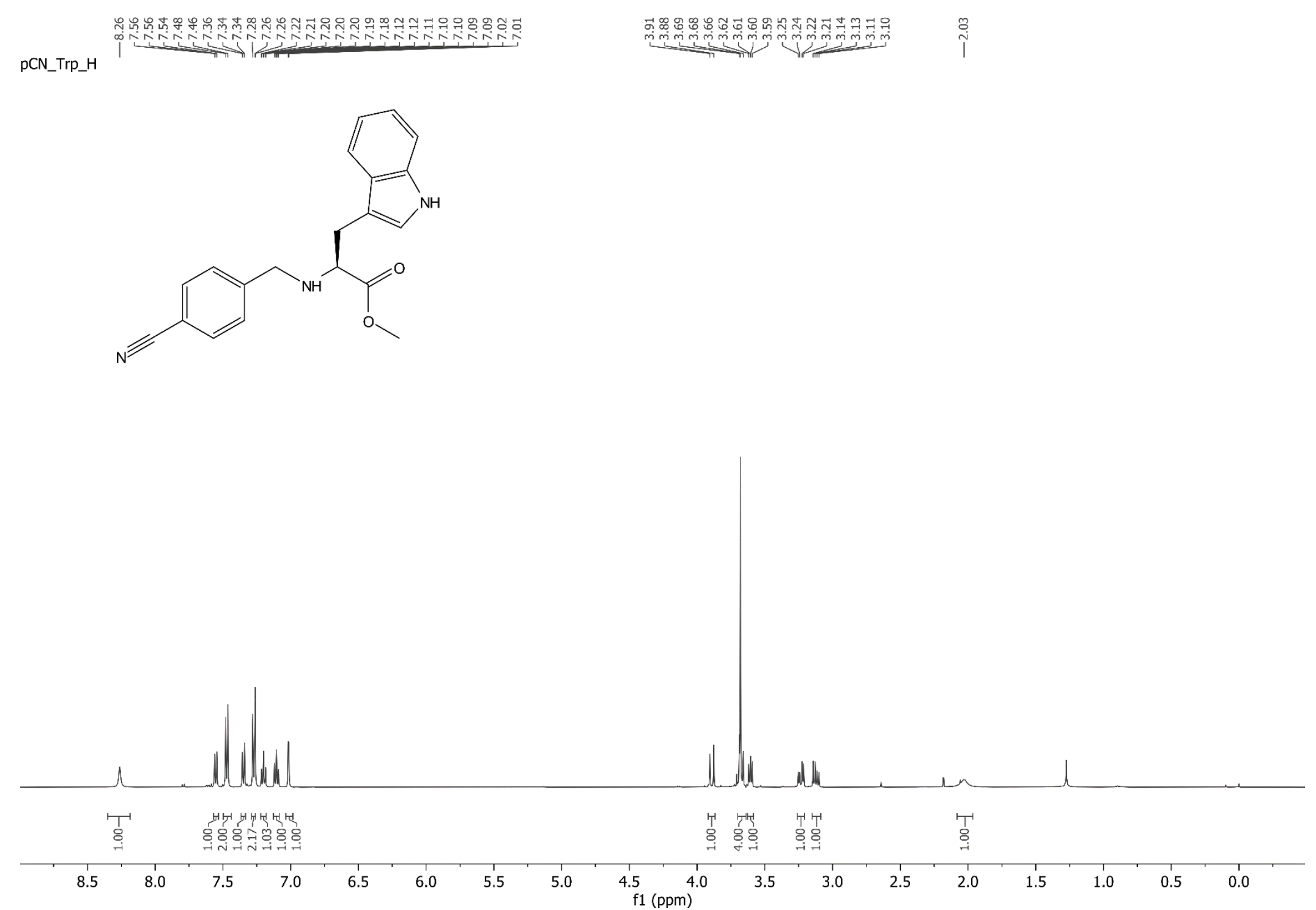


${ }^{13} \mathrm{C} \mathrm{NMR} \mathrm{(126} \mathrm{MHz,} \mathrm{CDCl}_{3}$ ) spectrum of 2q

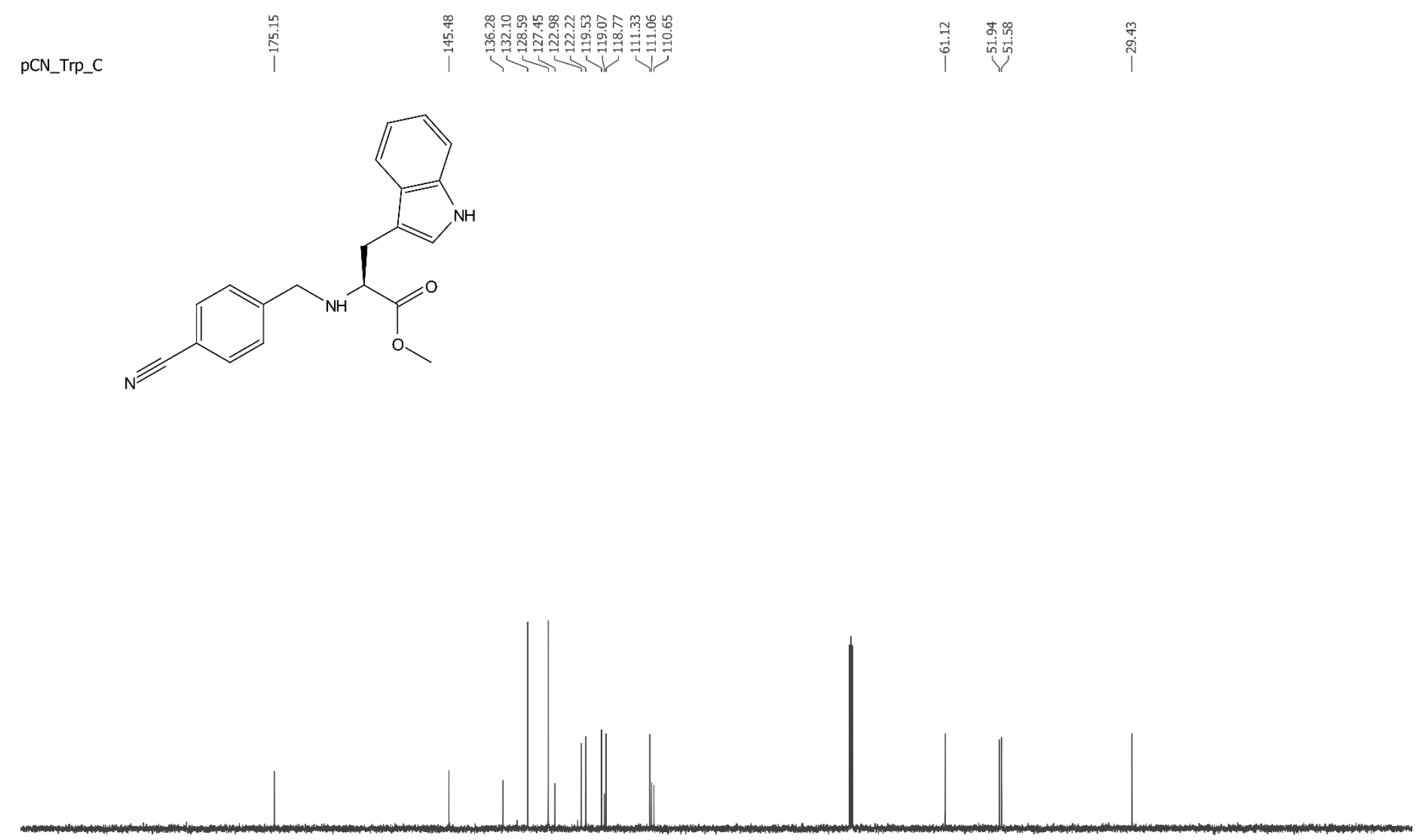

$\begin{array}{lllllll}210 & 200 & 190 & 180 & 170 & 160 & 150\end{array}$

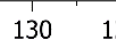

$\begin{array}{lll}120 & 110 & 100\end{array}$

f1 (ppm) 


\section{${ }^{1} \mathrm{H} \mathrm{NMR}\left(500 \mathrm{MHz}, \mathrm{CDCl}_{3}\right)$ spectrum of $\mathbf{2 r}$}
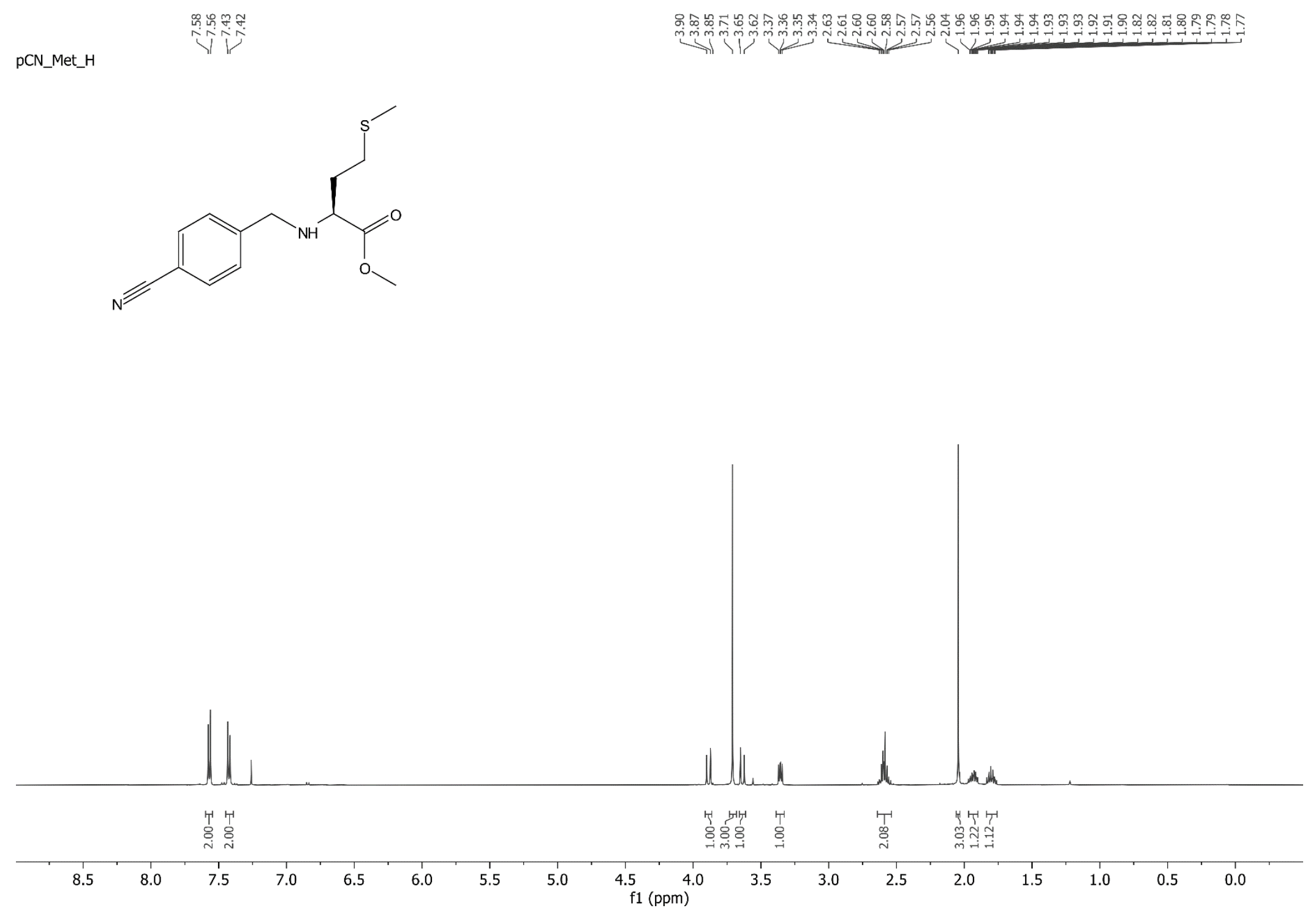
${ }^{13} \mathrm{C} \mathrm{NMR} \mathrm{(126} \mathrm{MHz,} \mathrm{CDCl}_{3}$ ) spectrum of $\mathbf{2 r}$

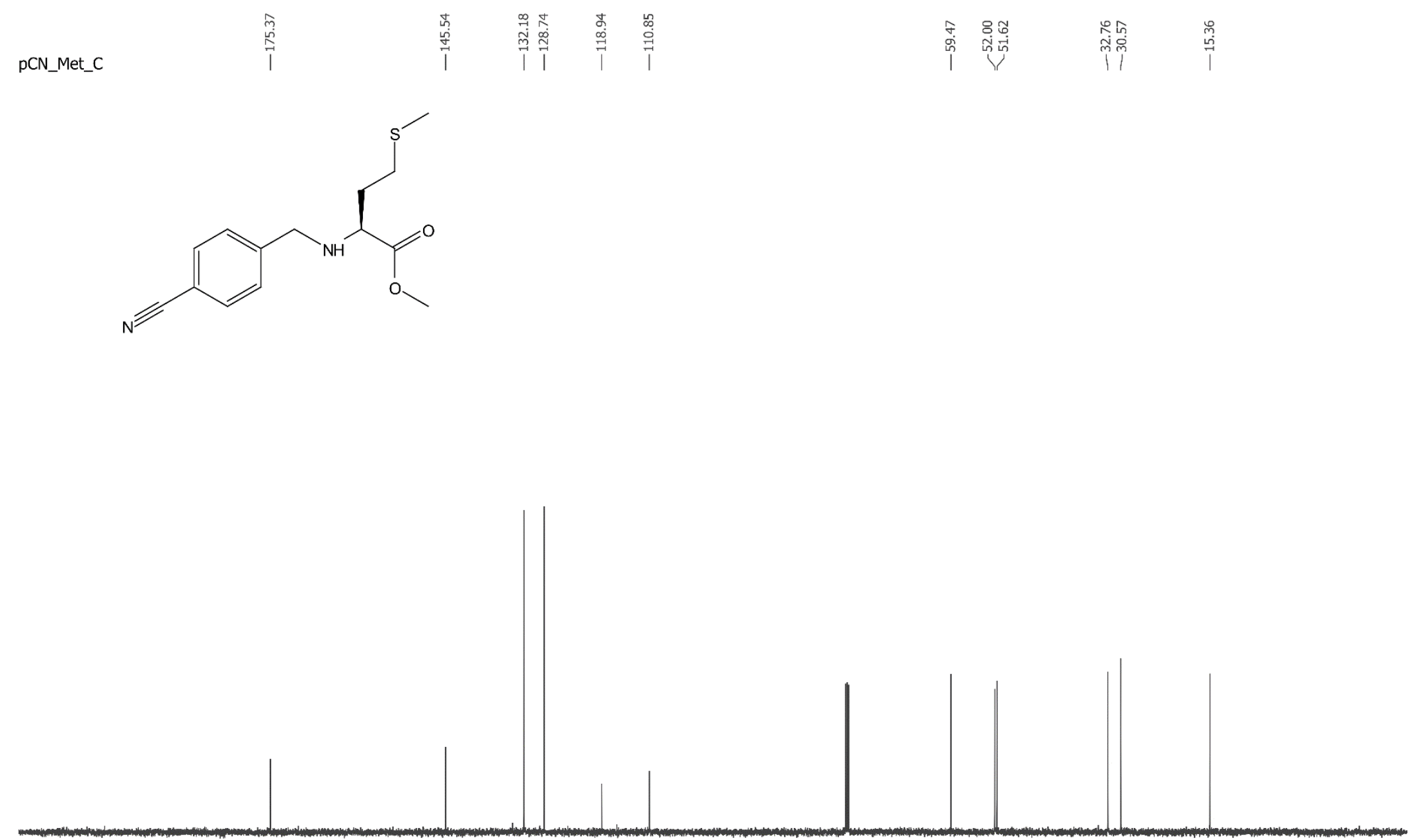

$\begin{array}{lllllllllllllllllllllllllll}210 & 200 & 190 & 180 & 170 & 160 & 150 & 140 & 130 & 120 & 110 & 100 & 90 & 80 & 70 & 60 & 50 & 40 & 30 & 20 & 10 & 0 & -10 & 10\end{array}$ 
${ }^{1} \mathrm{H}$ NMR $\left(400 \mathrm{MHz}, \mathrm{CDCl}_{3}\right)$ spectrum of $2 \mathrm{~s}$

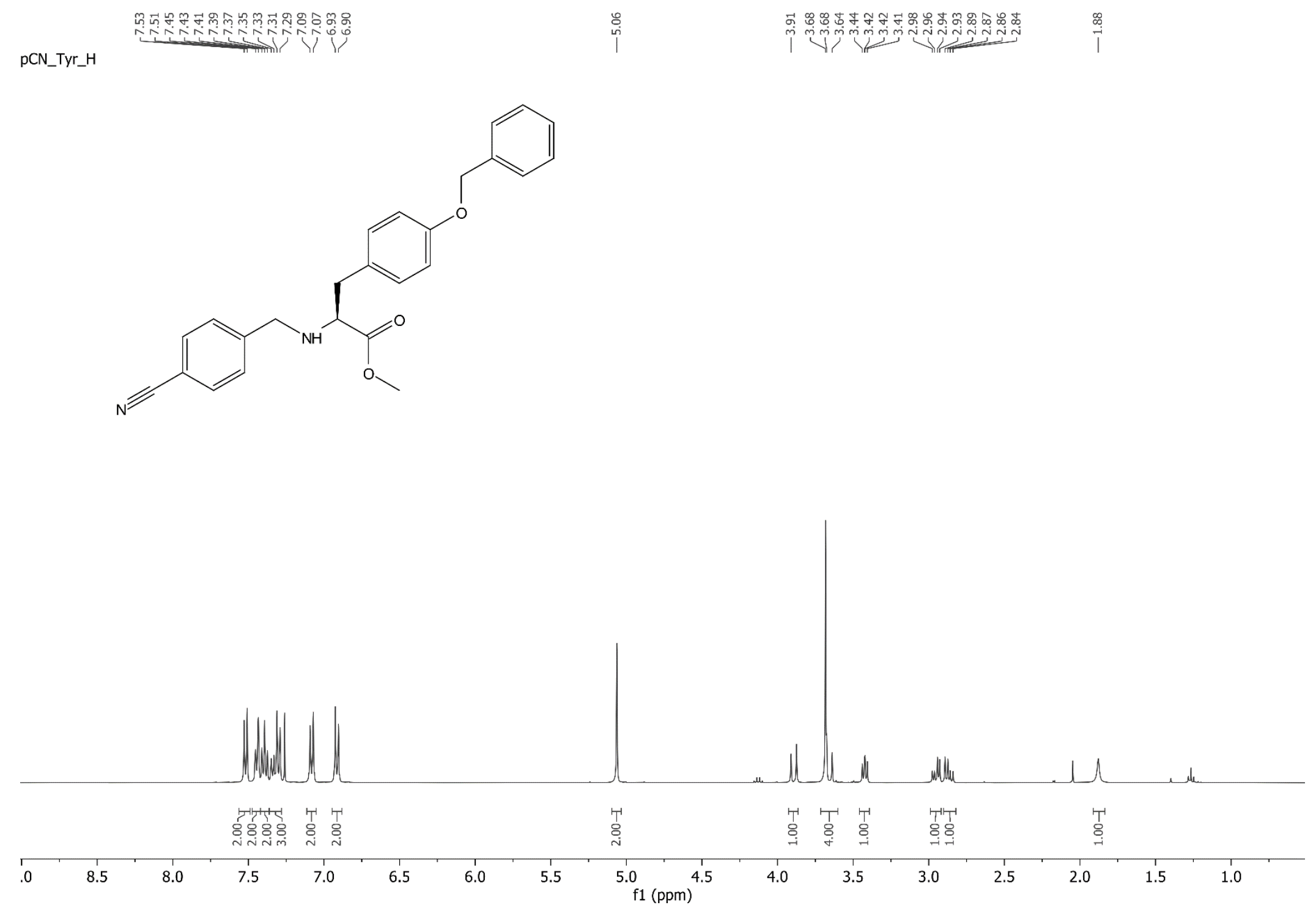


${ }^{13} \mathrm{C}$ NMR $\left(101 \mathrm{MHz}, \mathrm{CDCl}_{3}\right)$ spectrum of $\mathbf{2 s}$

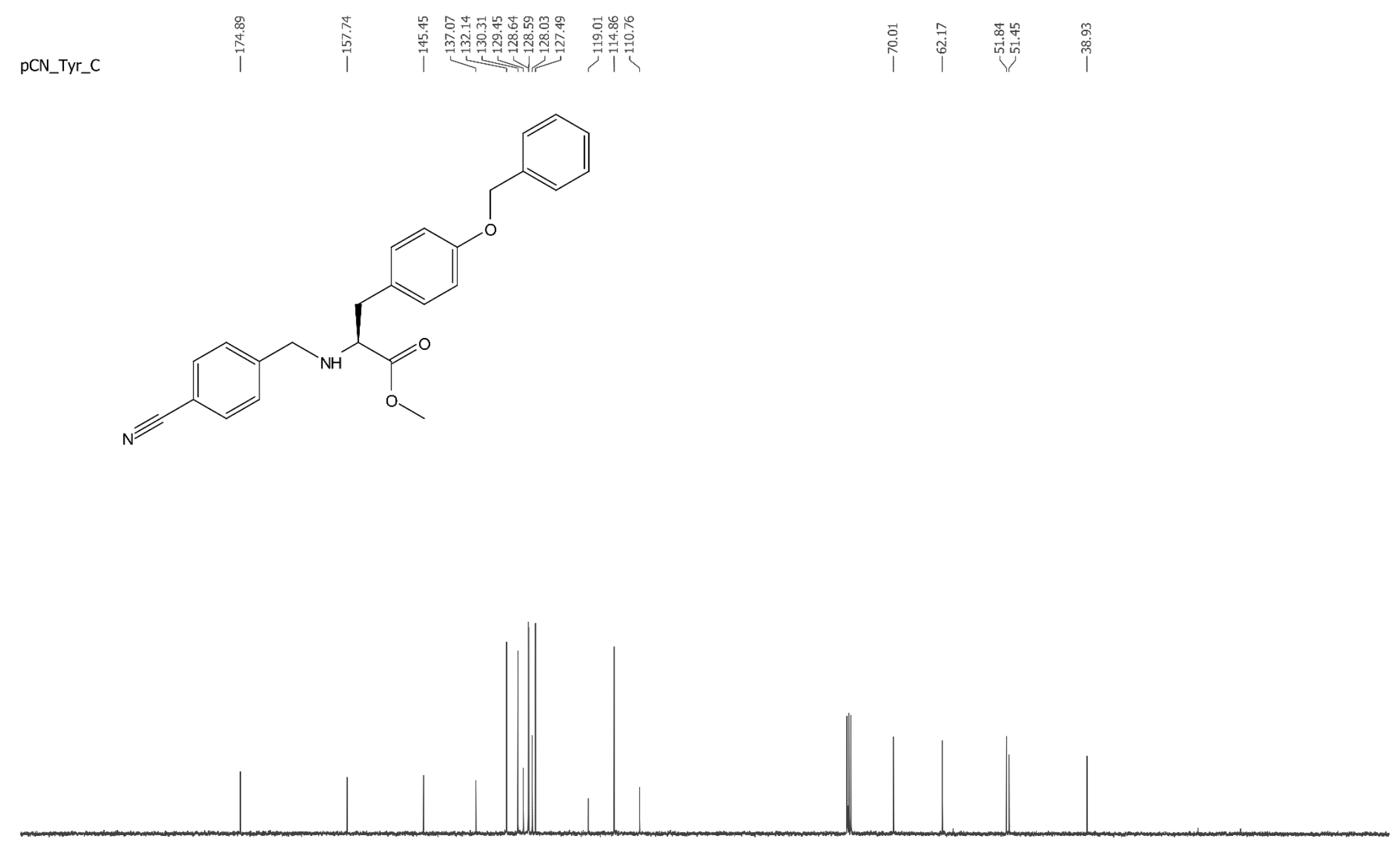

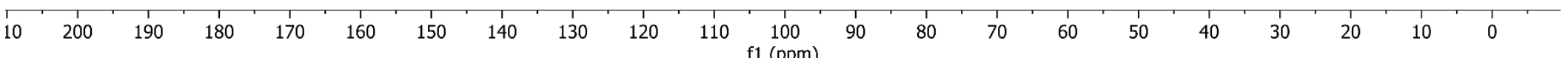


${ }^{1} \mathrm{H}$ NMR $\left(400 \mathrm{MHz}, \mathrm{CDCl}_{3}\right)$ spectrum of $\mathbf{2 t}$

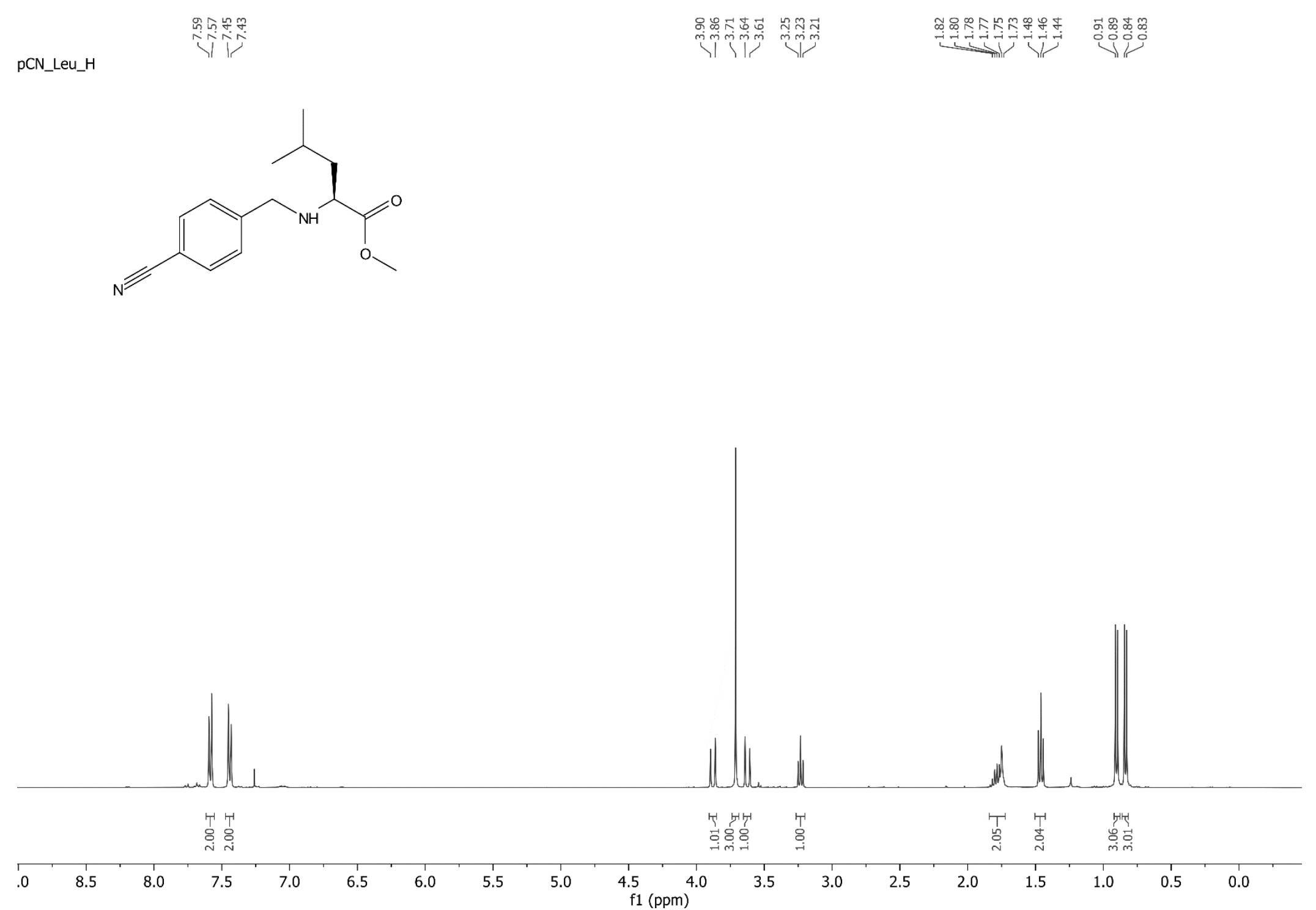


${ }^{13} \mathrm{C} \mathrm{NMR}\left(101 \mathrm{MHz}, \mathrm{CDCl}_{3}\right)$ spectrum of $\mathbf{2 t}$

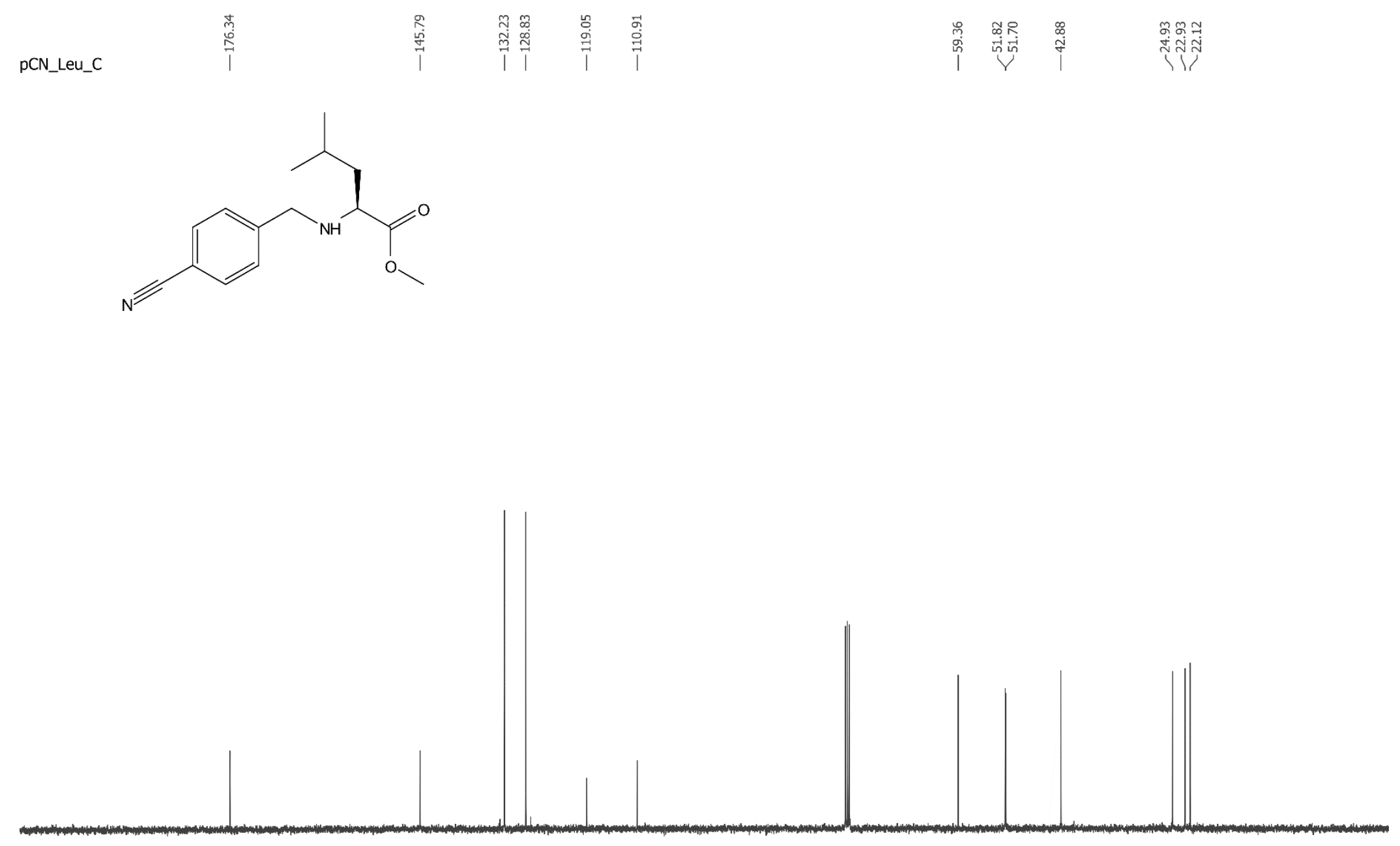

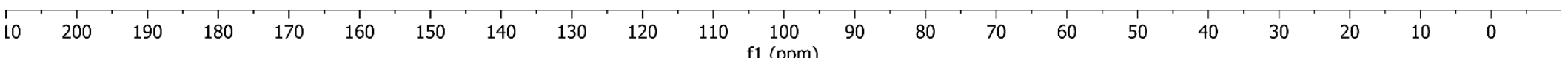


${ }^{1} \mathrm{H}$ NMR $\left(400 \mathrm{MHz}, \mathrm{CDCl}_{3}\right)$ spectrum of $\mathbf{2 u}$

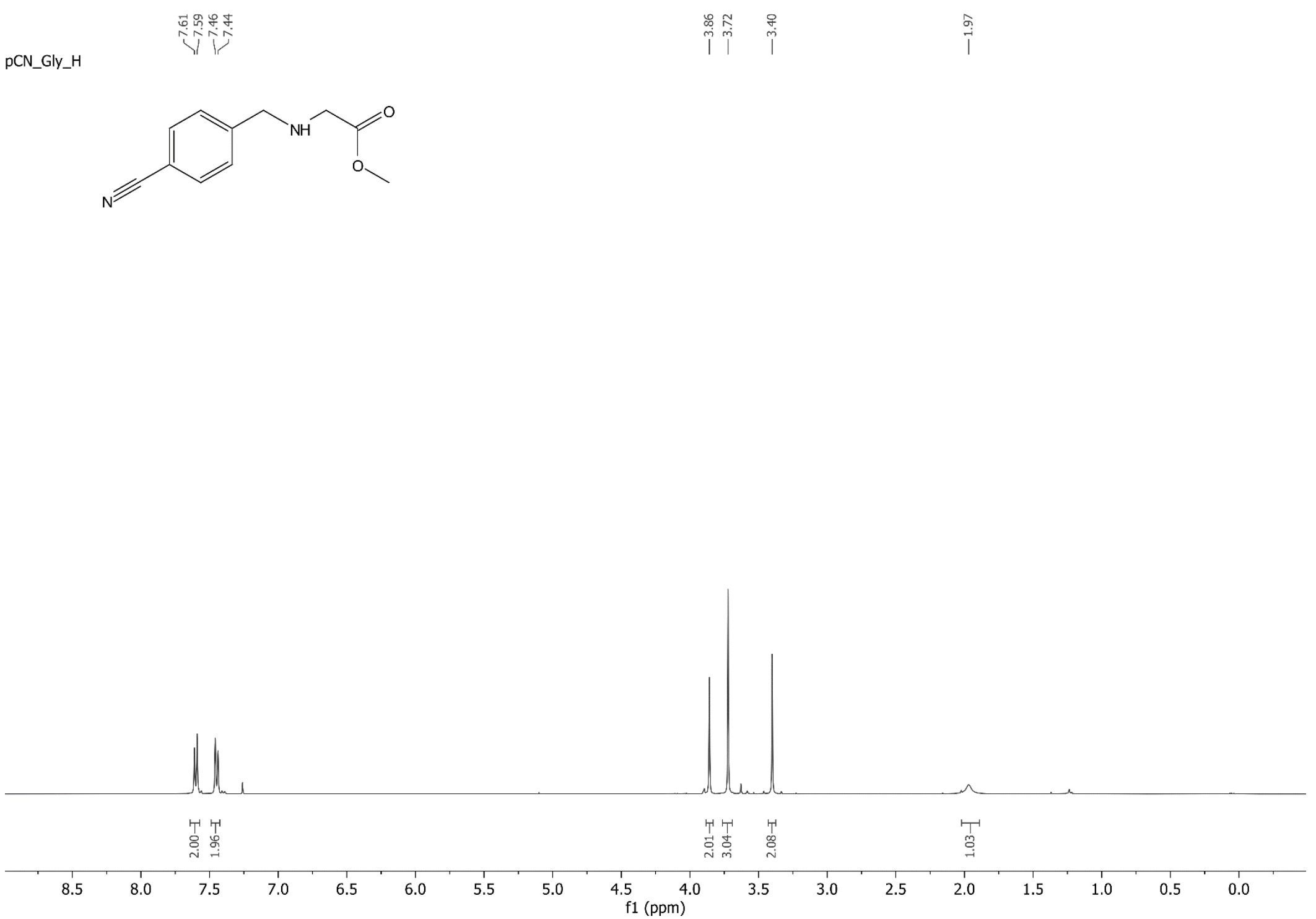


${ }^{13} \mathrm{C} \mathrm{NMR}\left(101 \mathrm{MHz}, \mathrm{CDCl}_{3}\right)$ spectrum of $\mathbf{2 u}$
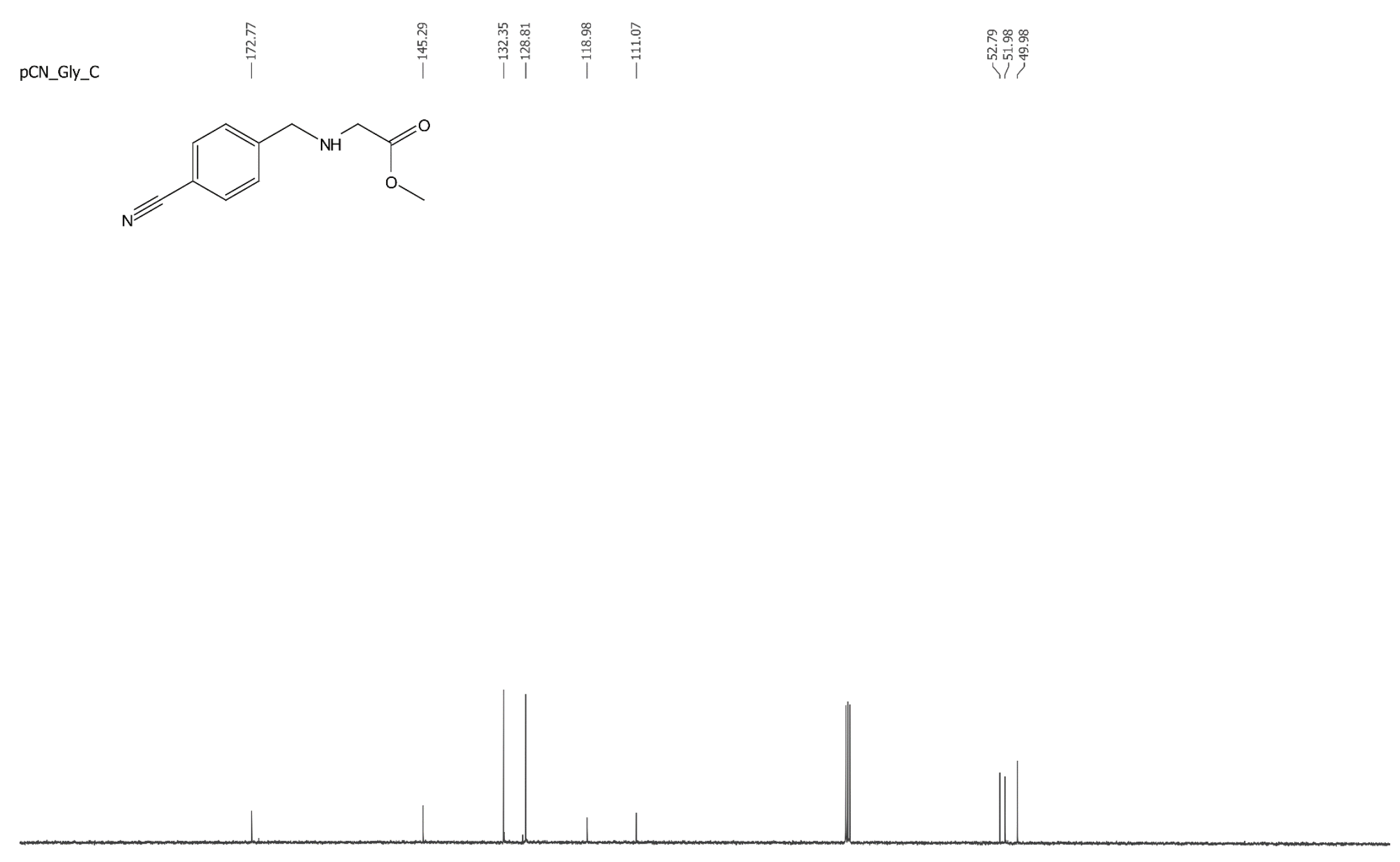

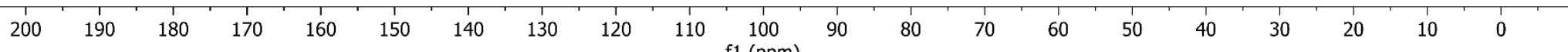


${ }^{1} \mathrm{H}$ NMR $\left(500 \mathrm{MHz}, \mathrm{CDCl}_{3}\right.$ ) spectrum of $\mathbf{2 v}$

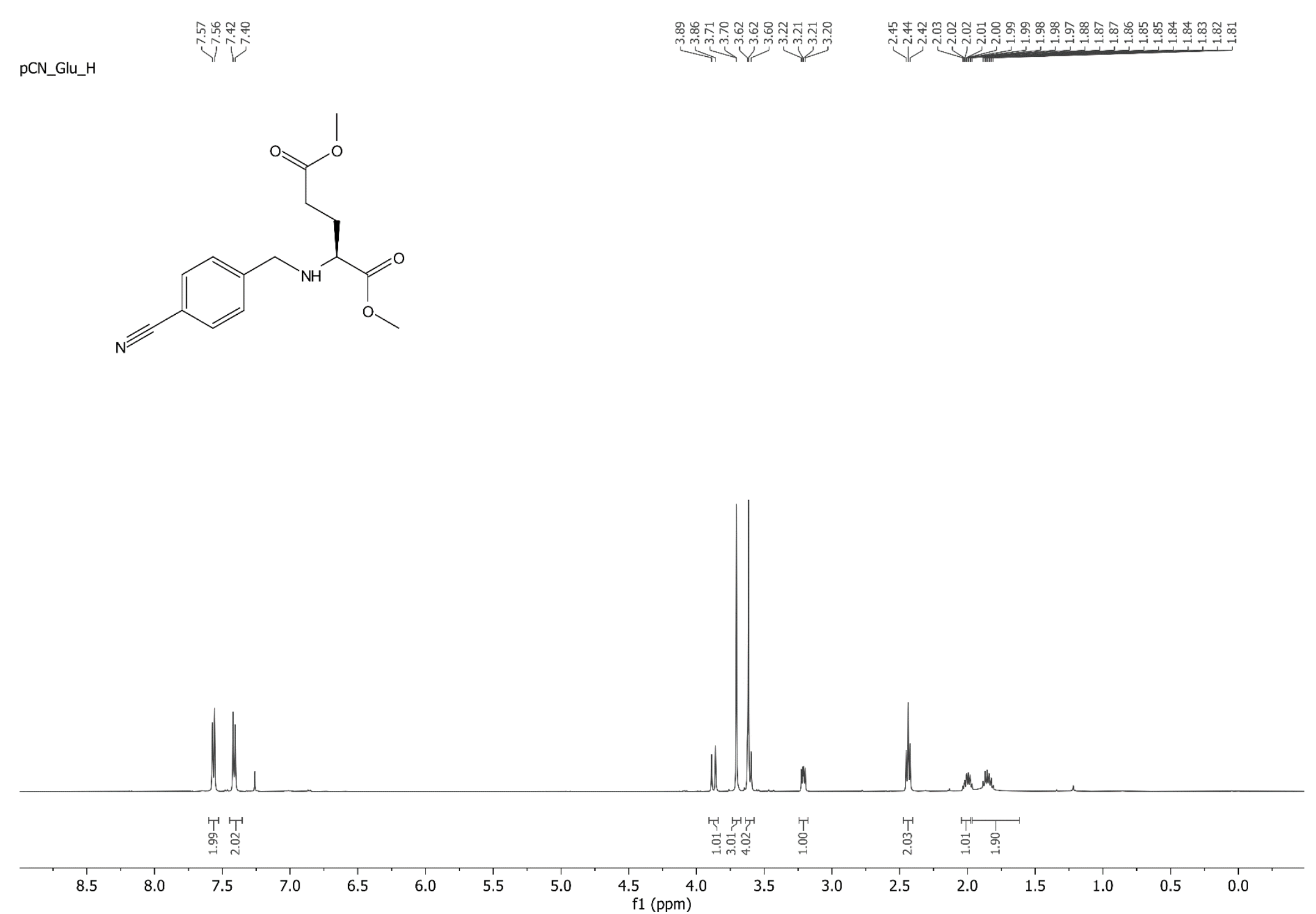


${ }^{13} \mathrm{C} \mathrm{NMR} \mathrm{(126} \mathrm{MHz,} \mathrm{CDCl}_{3}$ ) spectrum of $\mathbf{2 v}$

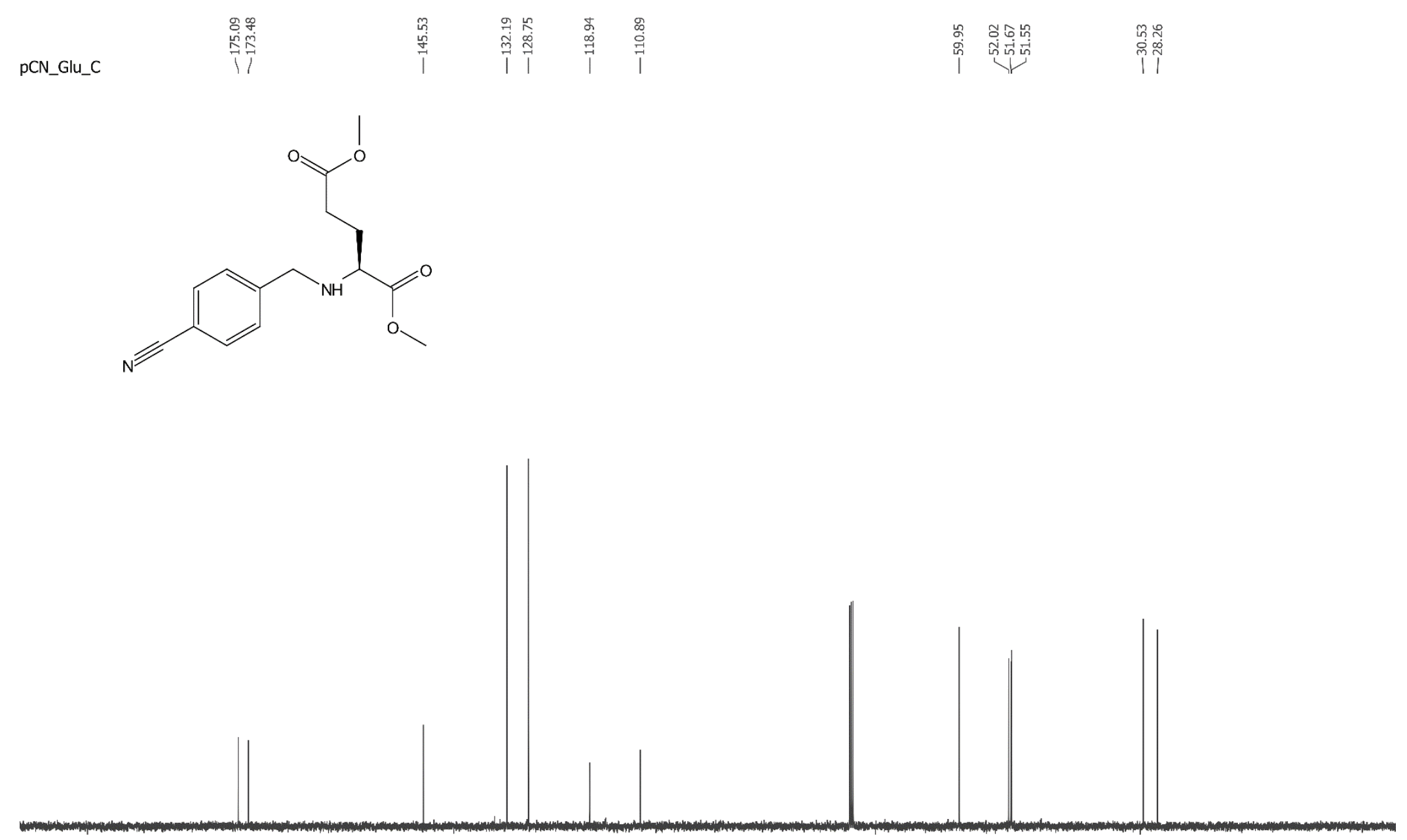

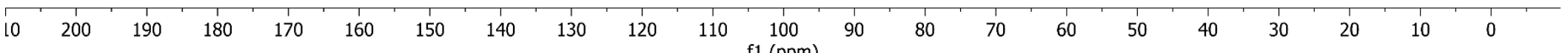


${ }^{1} \mathrm{H}$ NMR $\left(400 \mathrm{MHz}, \mathrm{CDCl}_{3}\right)$ spectrum of $\mathbf{2 w}$

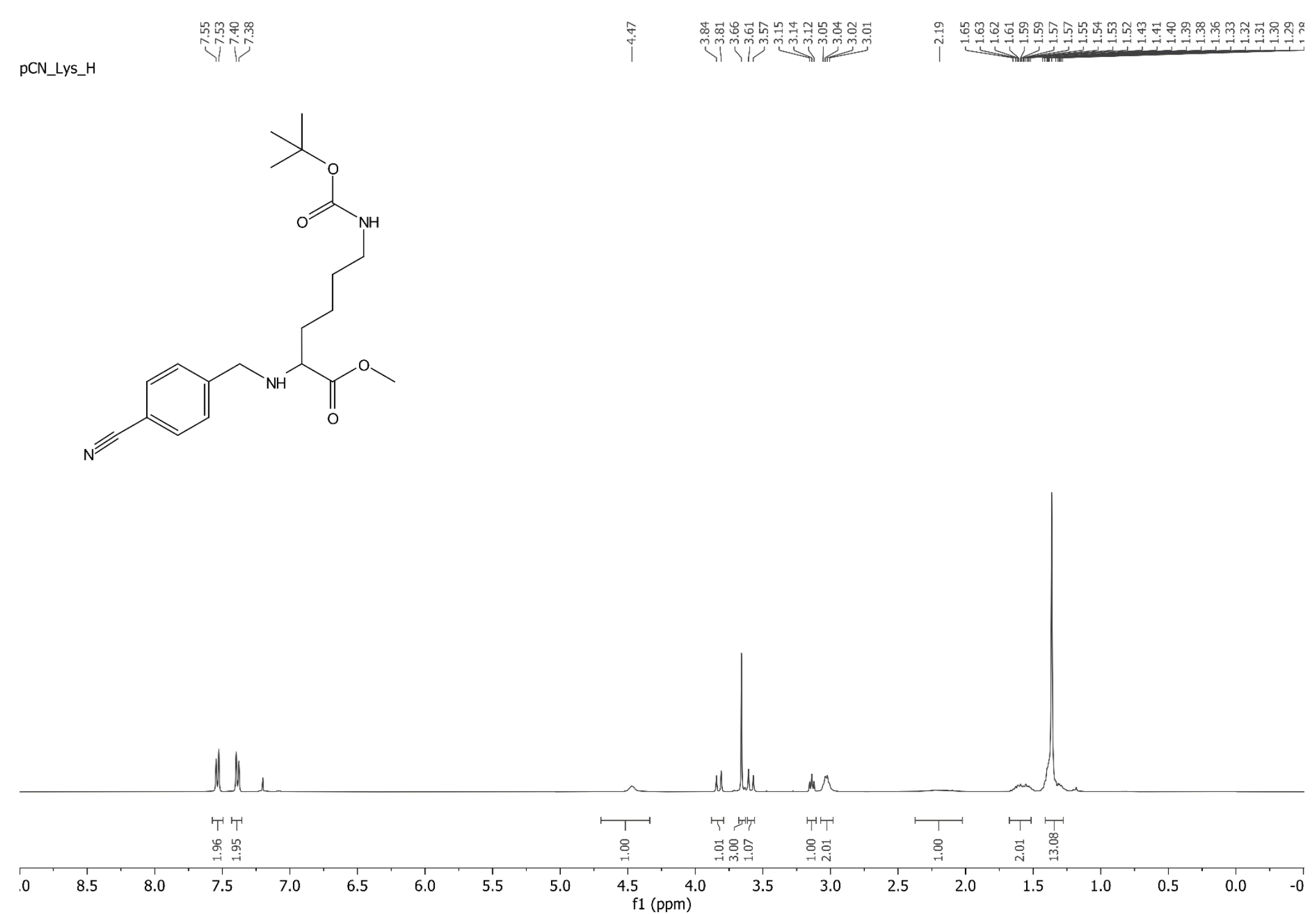


${ }^{13} \mathrm{C}$ NMR $\left(101 \mathrm{MHz}, \mathrm{CDCl}_{3}\right)$ spectrum of $2 \mathbf{w}$

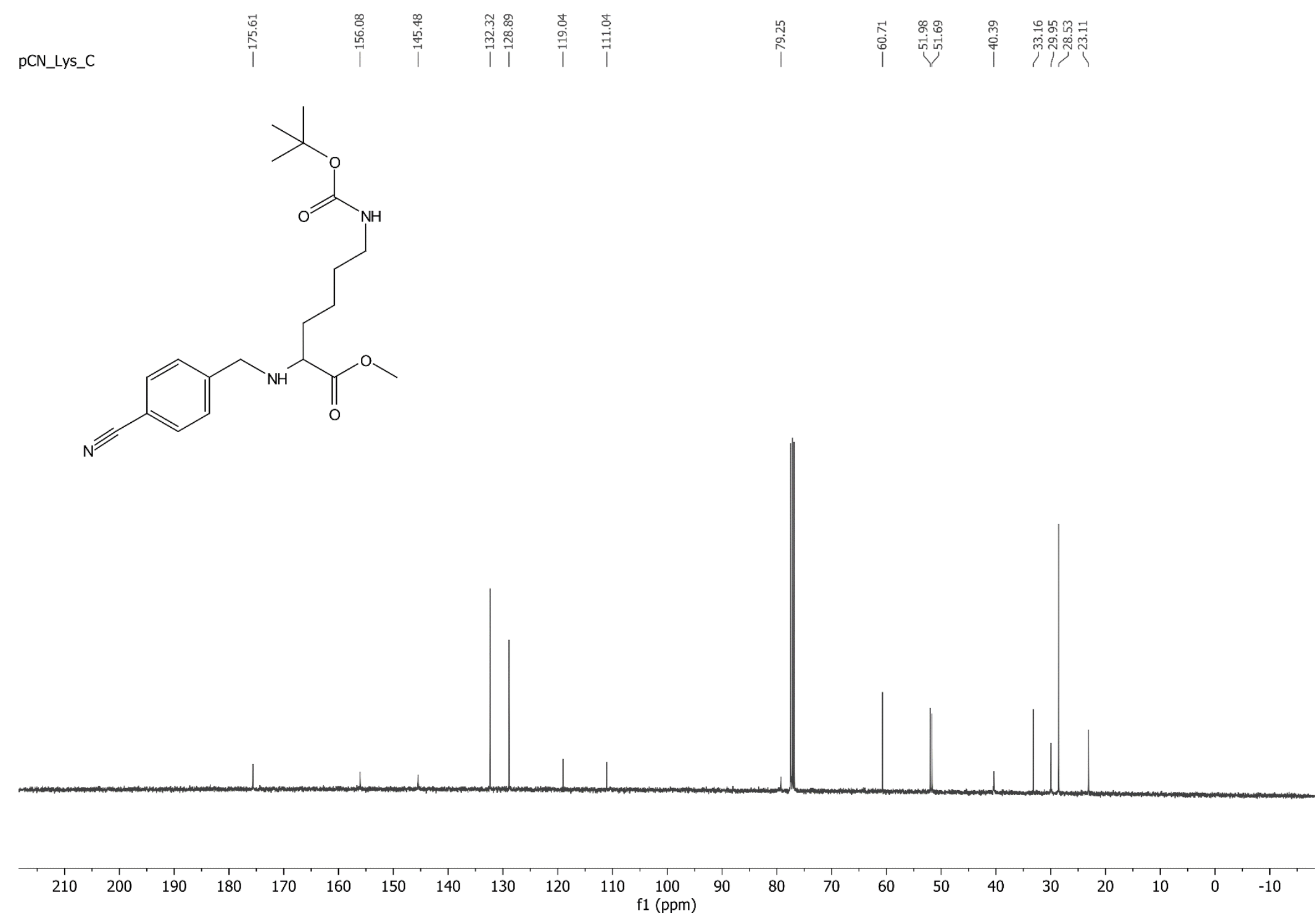


${ }^{1} \mathrm{H}$ NMR $\left(600 \mathrm{MHz}, \mathrm{CDCl}_{3}\right)$ spectrum of $\mathbf{2 x}$

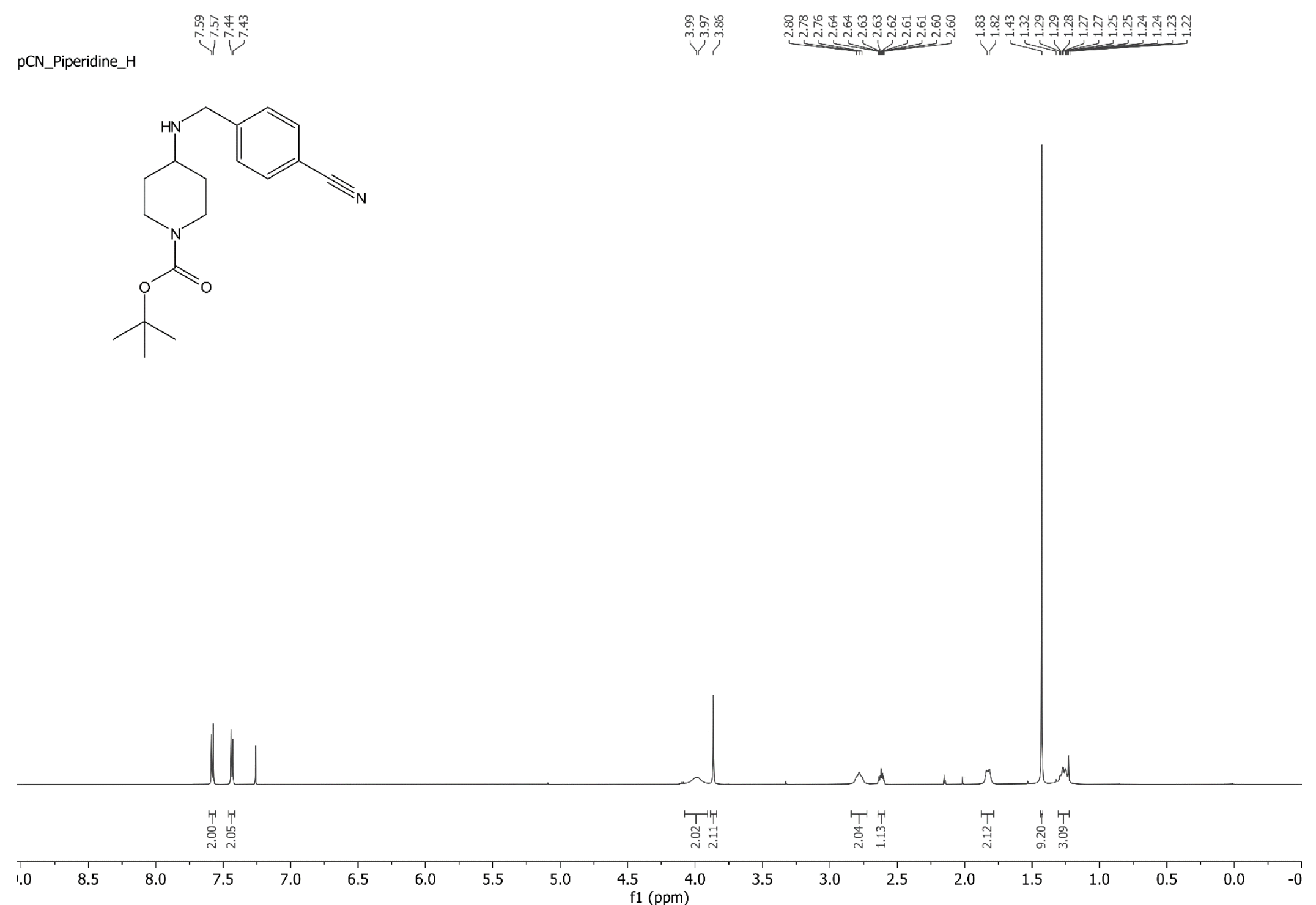


${ }^{13} \mathrm{C} \mathrm{NMR} \mathrm{(151} \mathrm{MHz,} \mathrm{CDCl}_{3}$ ) spectrum of $\mathbf{2 x}$
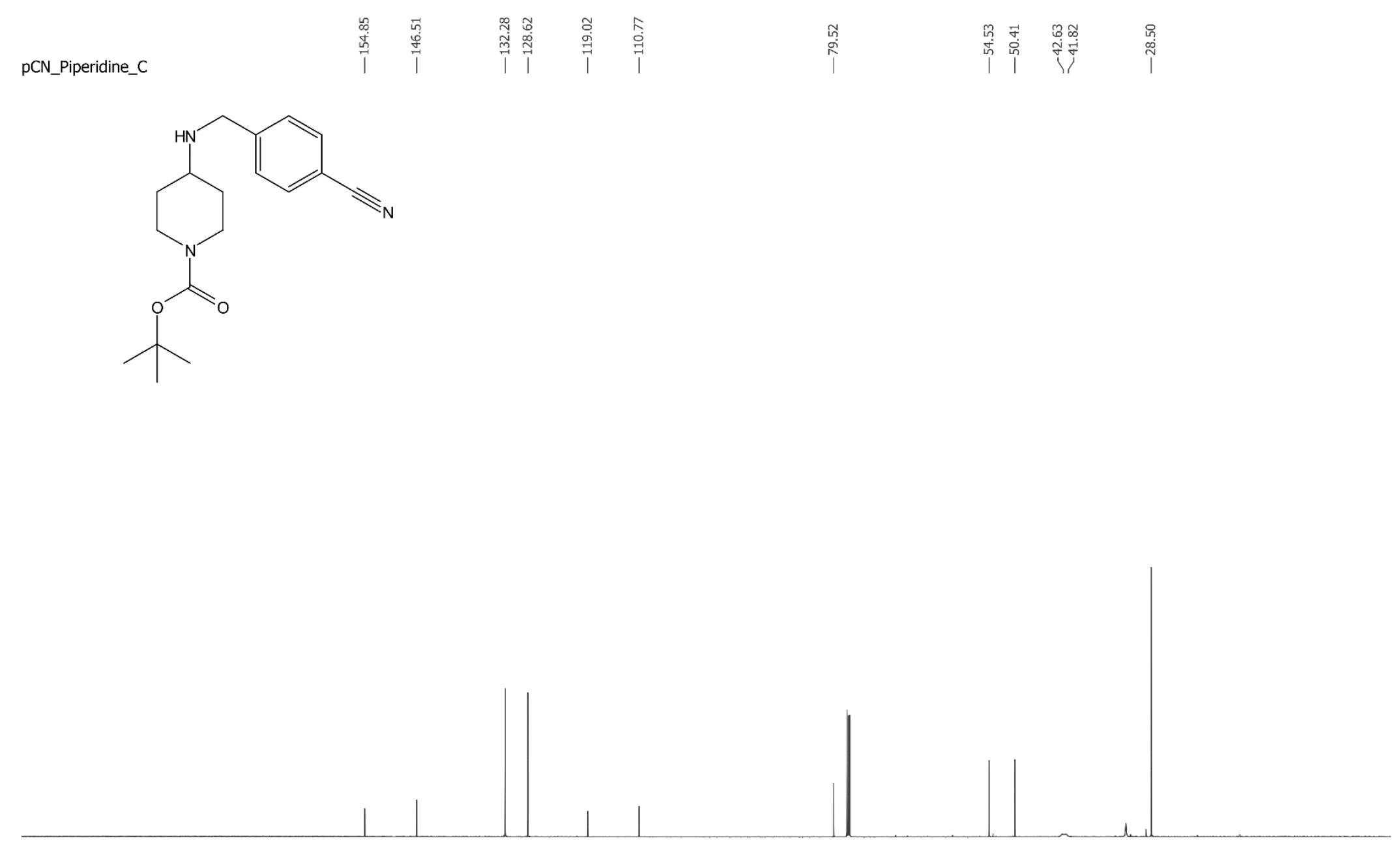

200

$\begin{array}{lllll}190 & 180 & 170 & 160 & 150\end{array}$

$\begin{array}{llllll}150 & 140 & 130 & 120 & 110 & 100\end{array}$ 


\section{${ }^{1} \mathrm{H}$ NMR $\left(500 \mathrm{MHz}, \mathrm{CDCl}_{3}\right)$ spectrum of $\mathbf{2 y}$}
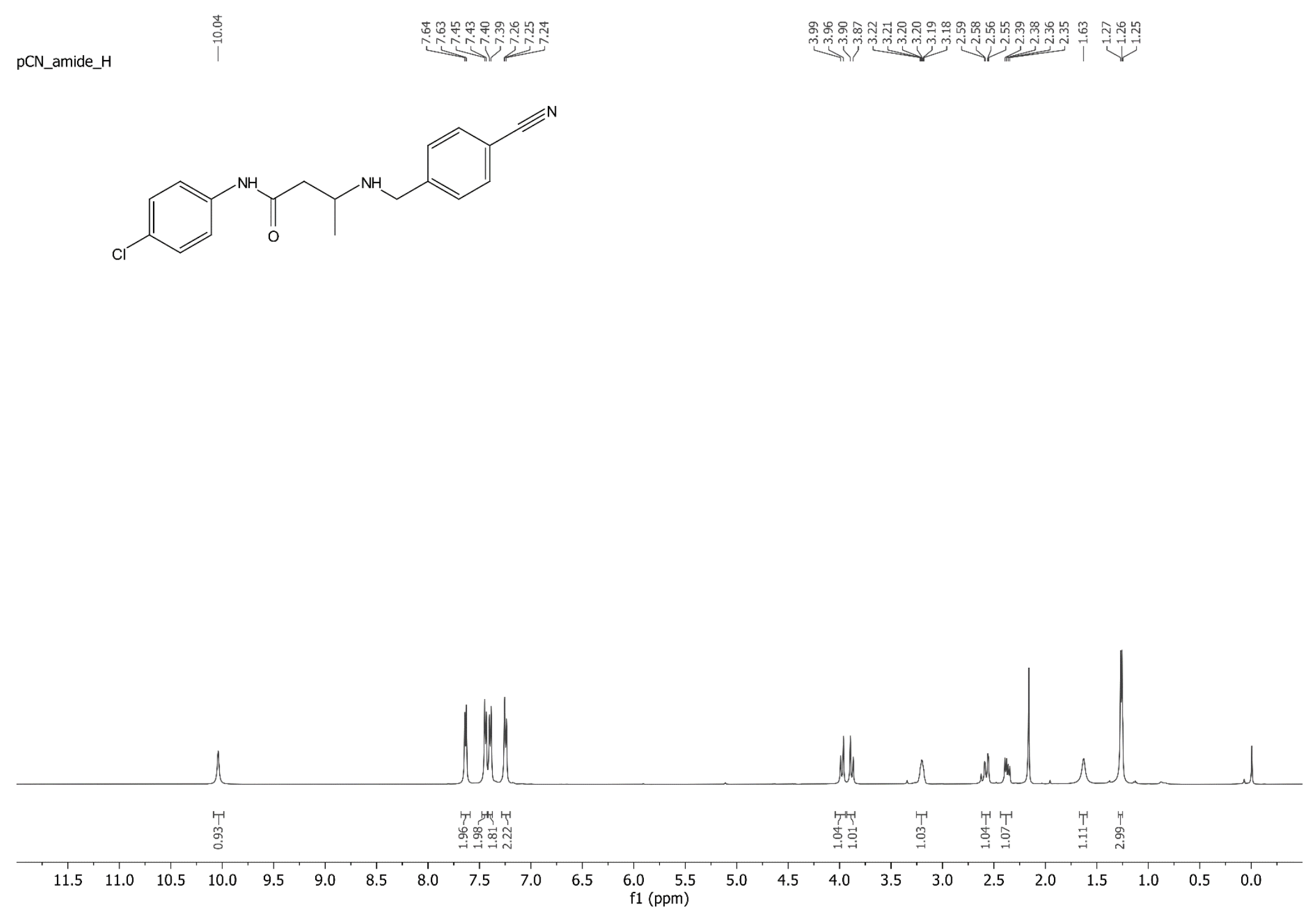
${ }^{13} \mathrm{C}$ NMR $\left(126 \mathrm{MHz}, \mathrm{CDCl}_{3}\right.$ ) spectrum of $\mathbf{2 y}$

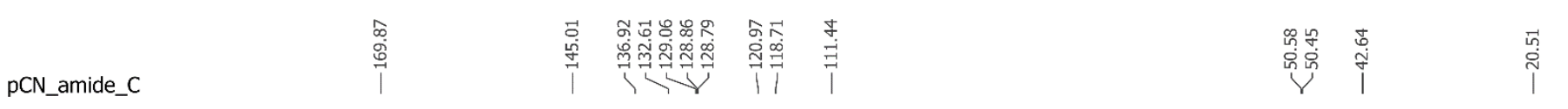
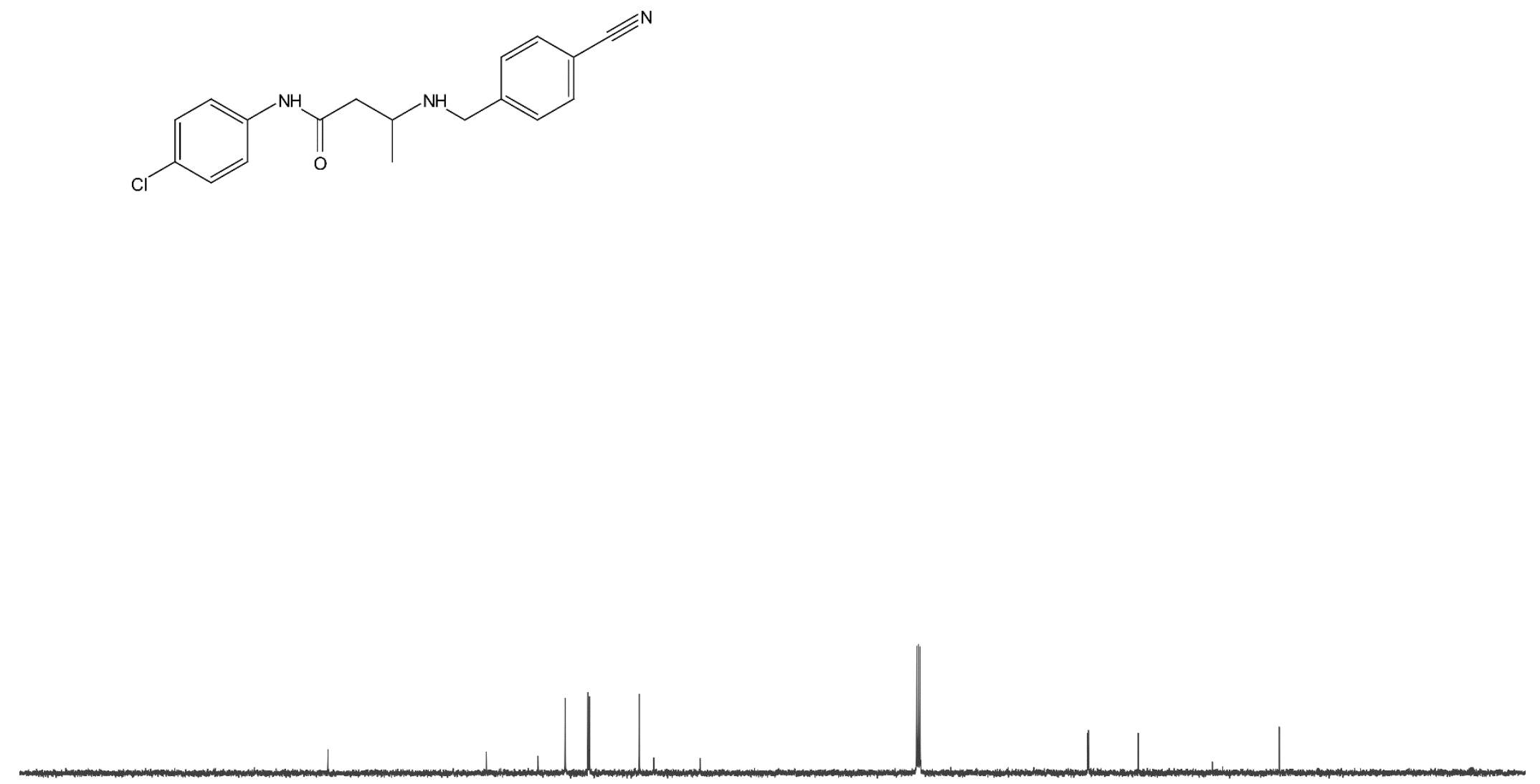

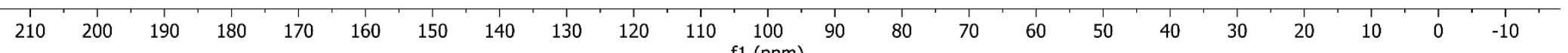


${ }^{1} \mathrm{H}$ NMR $\left(400 \mathrm{MHz}, \mathrm{CDCl}_{3}\right)$ spectrum of $\mathbf{2 z}$

pCN_Aspartame_H

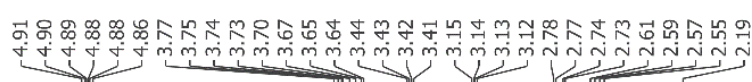

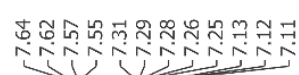<smiles>COC(=O)C[C@H](NCc1ccc(C#N)cc1)C(=O)N[C@@H](Cc1ccccc1)C(=O)OC</smiles>

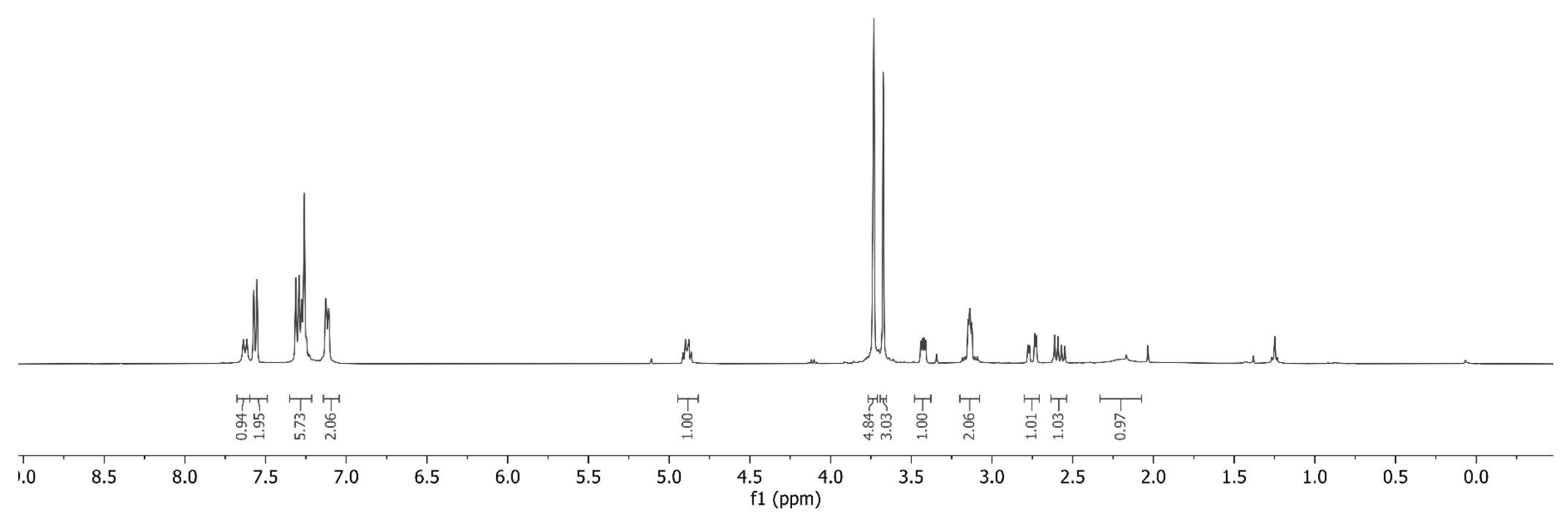


${ }^{13} \mathrm{C} \mathrm{NMR} \mathrm{(101} \mathrm{MHz,} \mathrm{CDCl}_{3}$ ) spectrum of $\mathbf{2 z}$

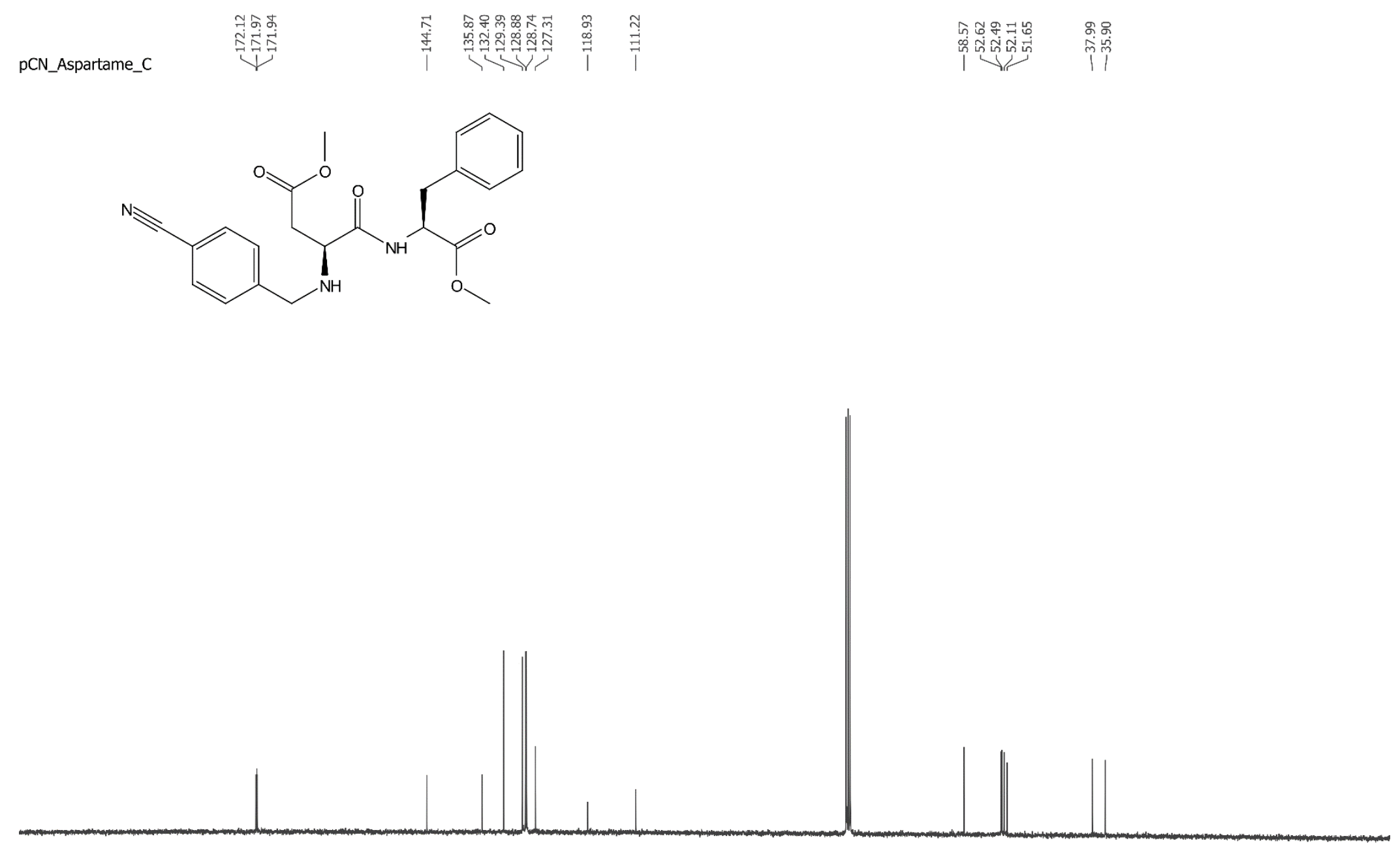

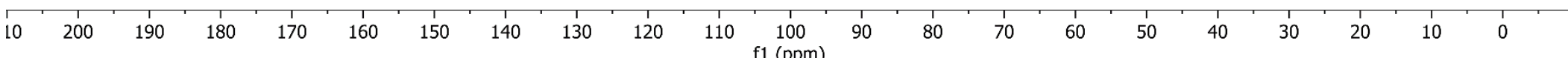


${ }^{1} \mathrm{H}$ NMR $\left(500 \mathrm{MHz}, \mathrm{CDCl}_{3}\right)$ spectrum of 2aa

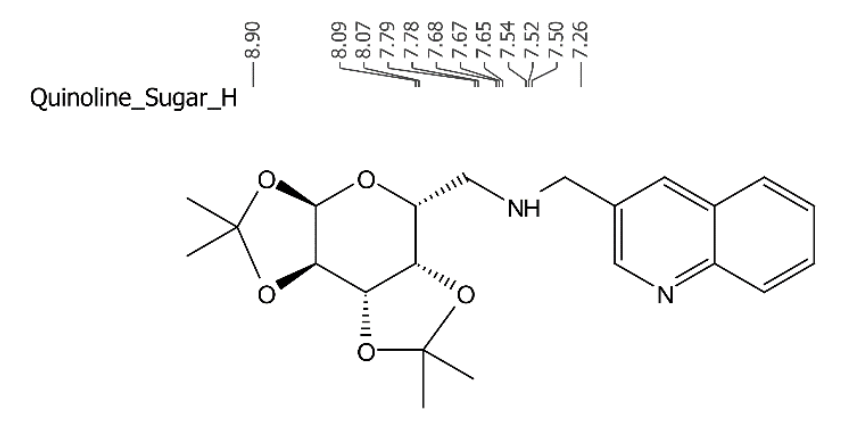

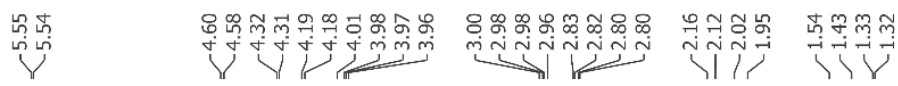

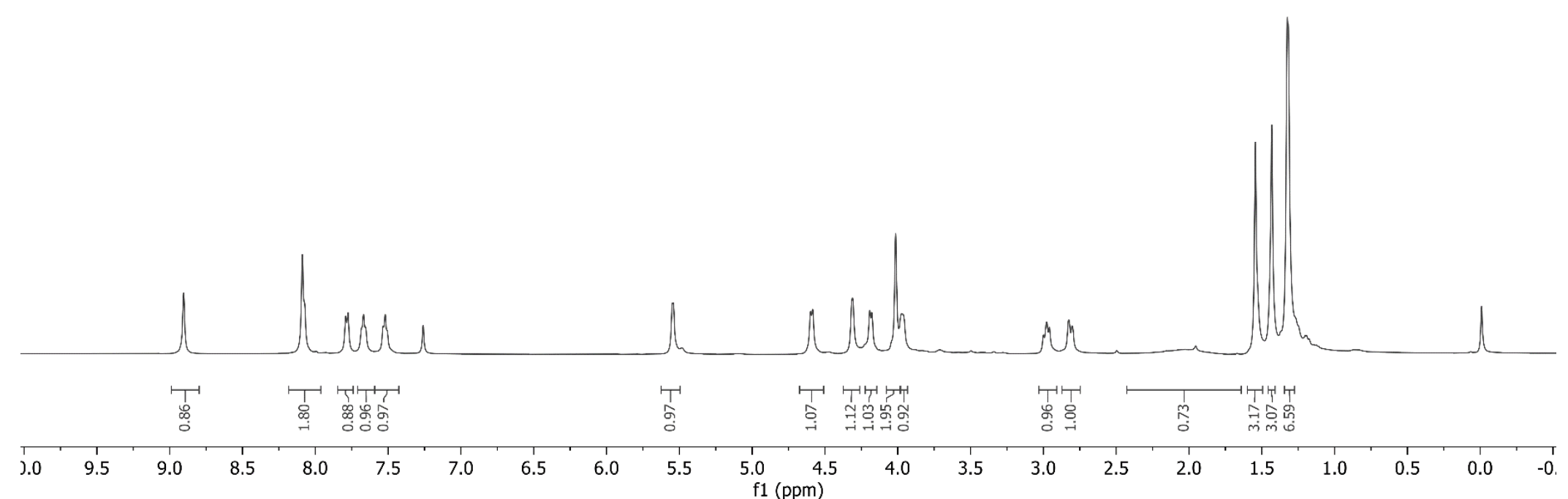


${ }^{13} \mathrm{C} \mathrm{NMR}\left(126 \mathrm{MHz}, \mathrm{CDCl}_{3}\right)$ spectrum of 2aa

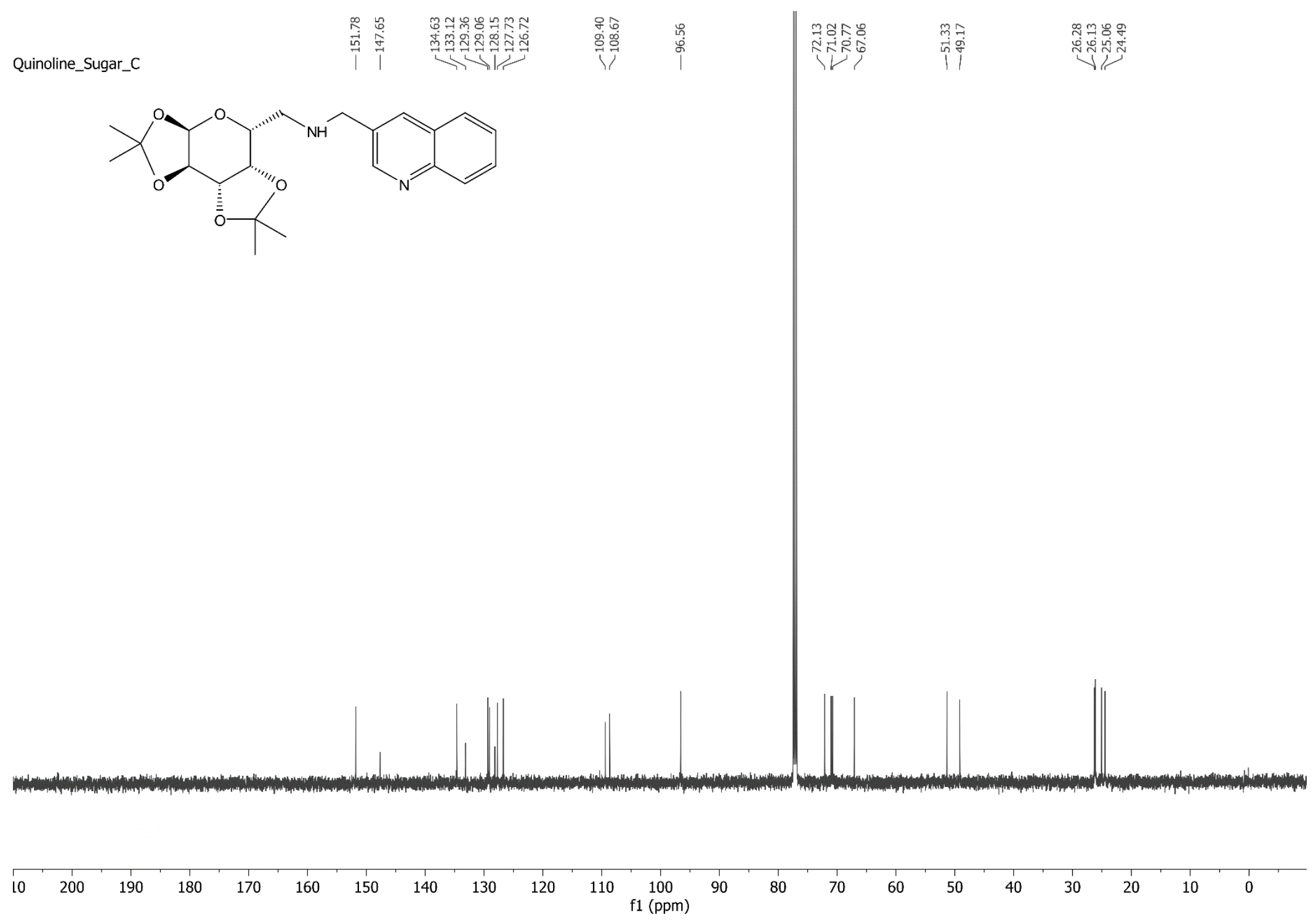


${ }^{1} \mathrm{H}$ NMR $\left(500 \mathrm{MHz}, \mathrm{CDCl}_{3}\right)$ spectrum of $\mathbf{3 a}$

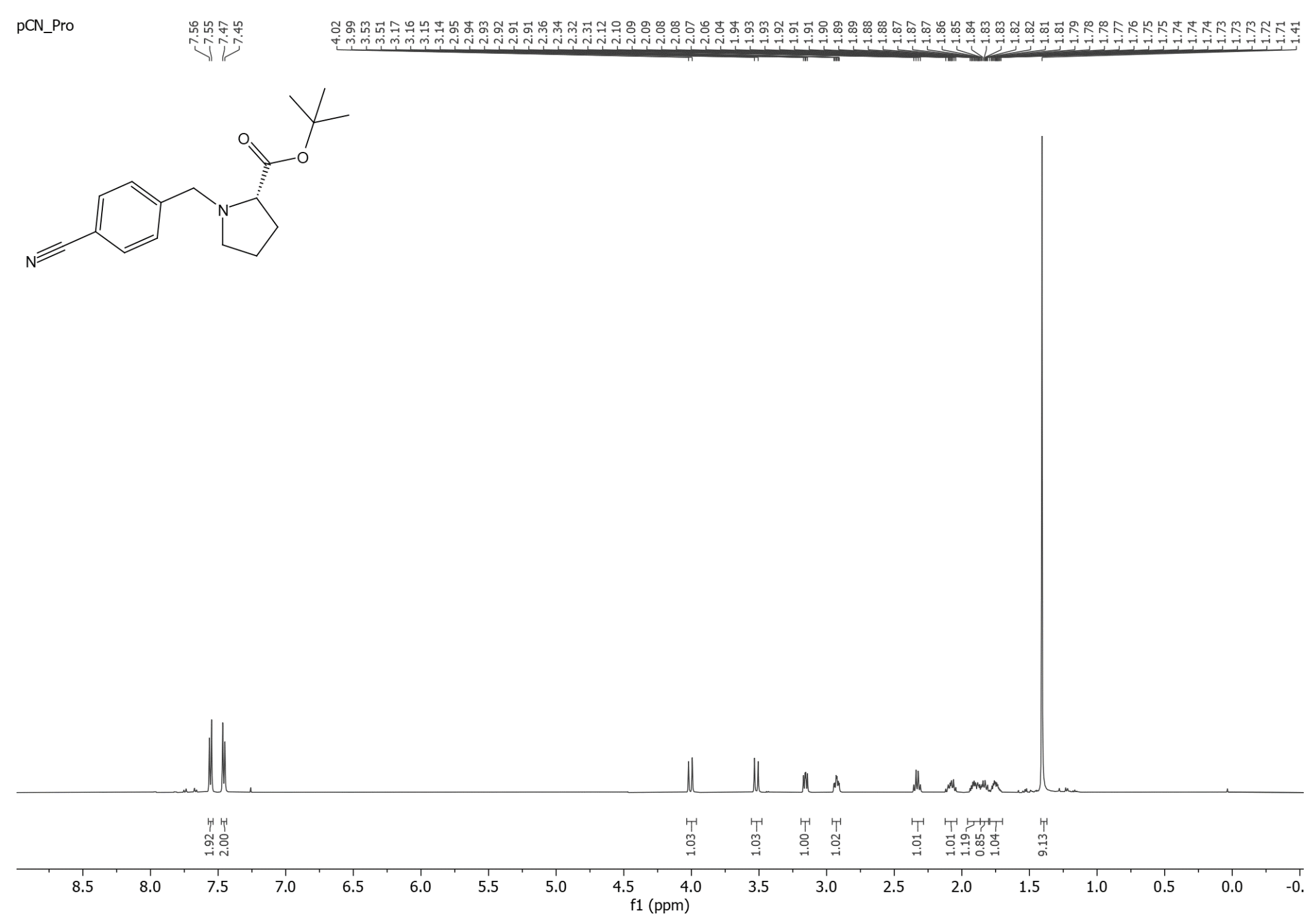


${ }^{13} \mathrm{C} \mathrm{NMR}\left(126 \mathrm{MHz}, \mathrm{CDCl}_{3}\right)$ spectrum of $\mathbf{3 a}$
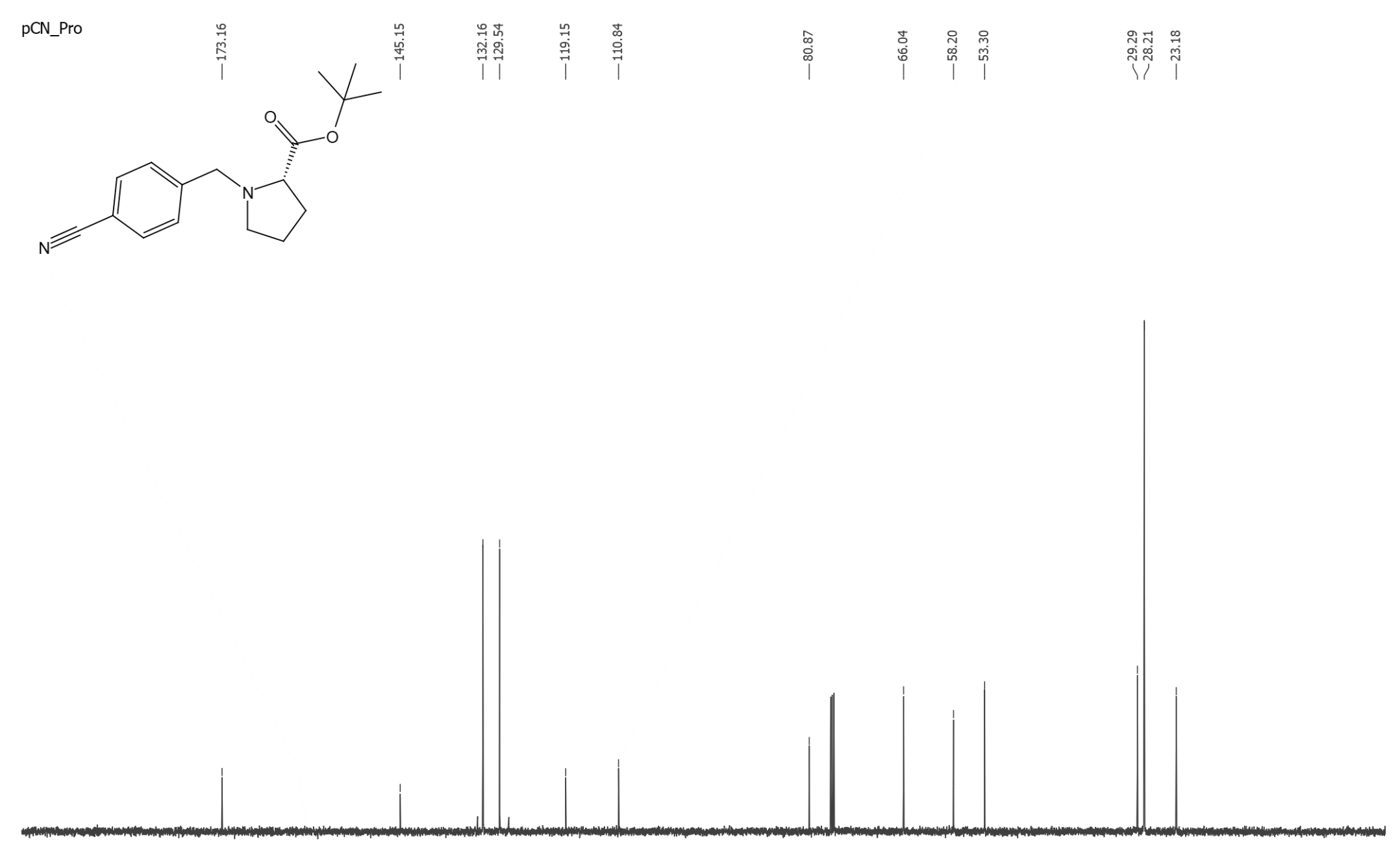
${ }^{1} \mathrm{H}$ NMR (400 MHz, $\left.\mathrm{CDCl}_{3}\right)$ spectrum of $\mathbf{3 b}$

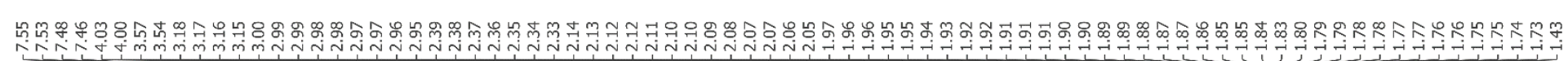
pCF3_Pro_H
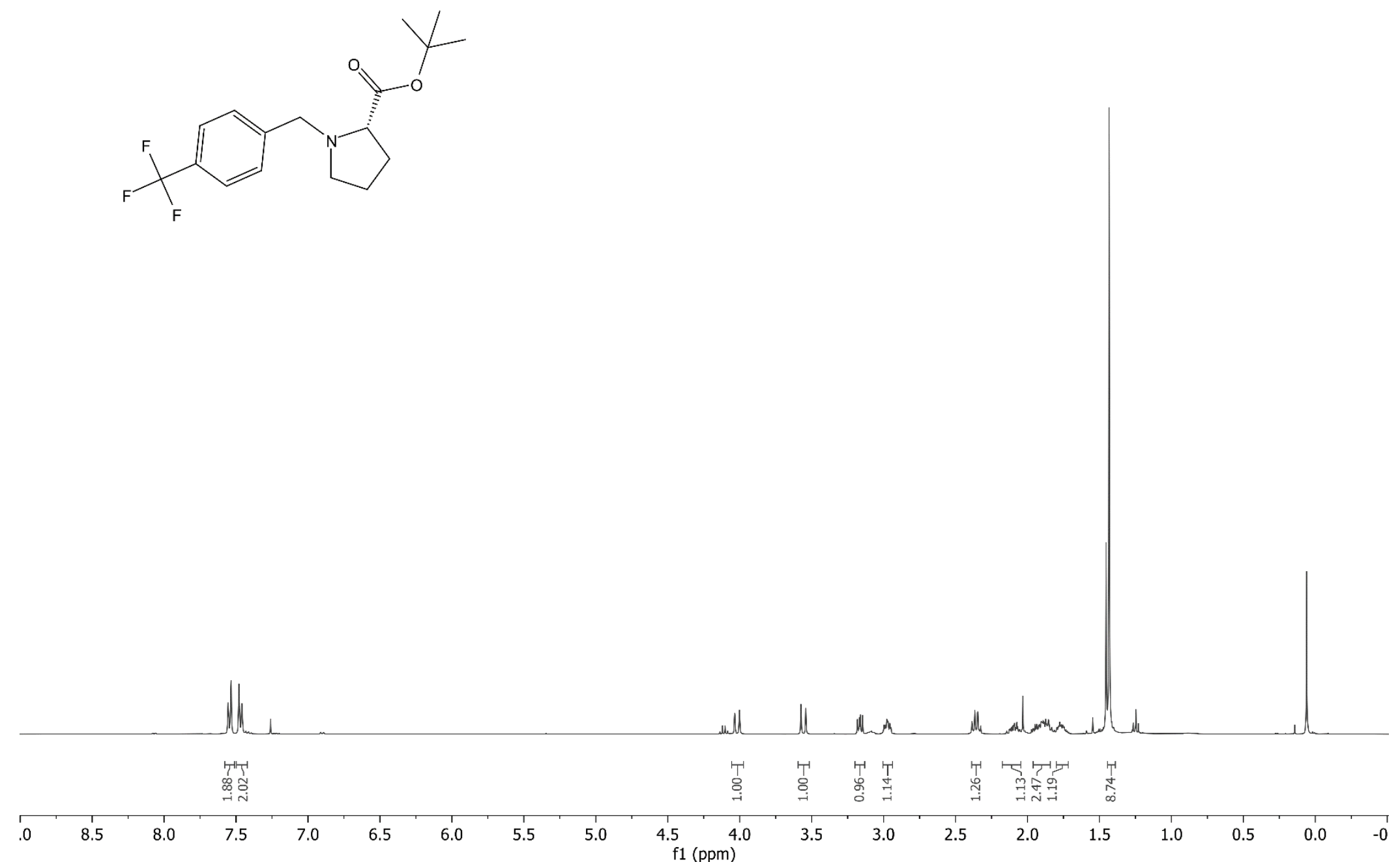
SUPPORTING INFORMATION

${ }^{13} \mathrm{C}$ NMR $\left(101 \mathrm{MHz}, \mathrm{CDCl}_{3}\right)$ spectrum of $\mathbf{3 b}$

PCF3_Pro_C

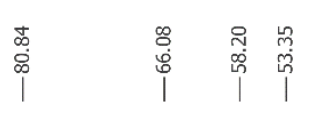

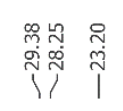
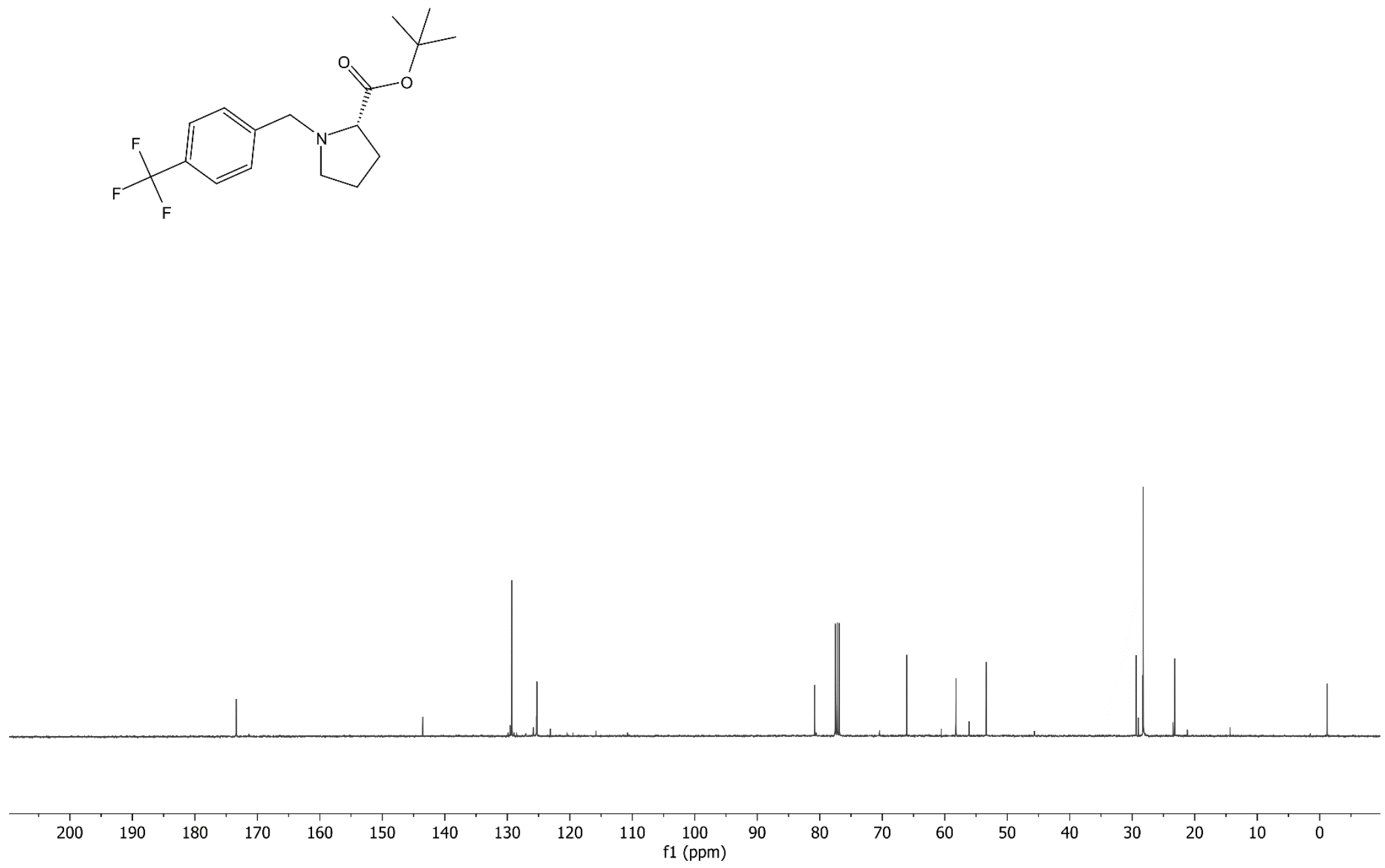

158 
$\left.{ }^{19} \mathrm{~F} \mathrm{NMR} \mathrm{(376} \mathrm{MHz,} \mathrm{CDCl}_{3}\right)$ spectrum of $\mathbf{3 b}$

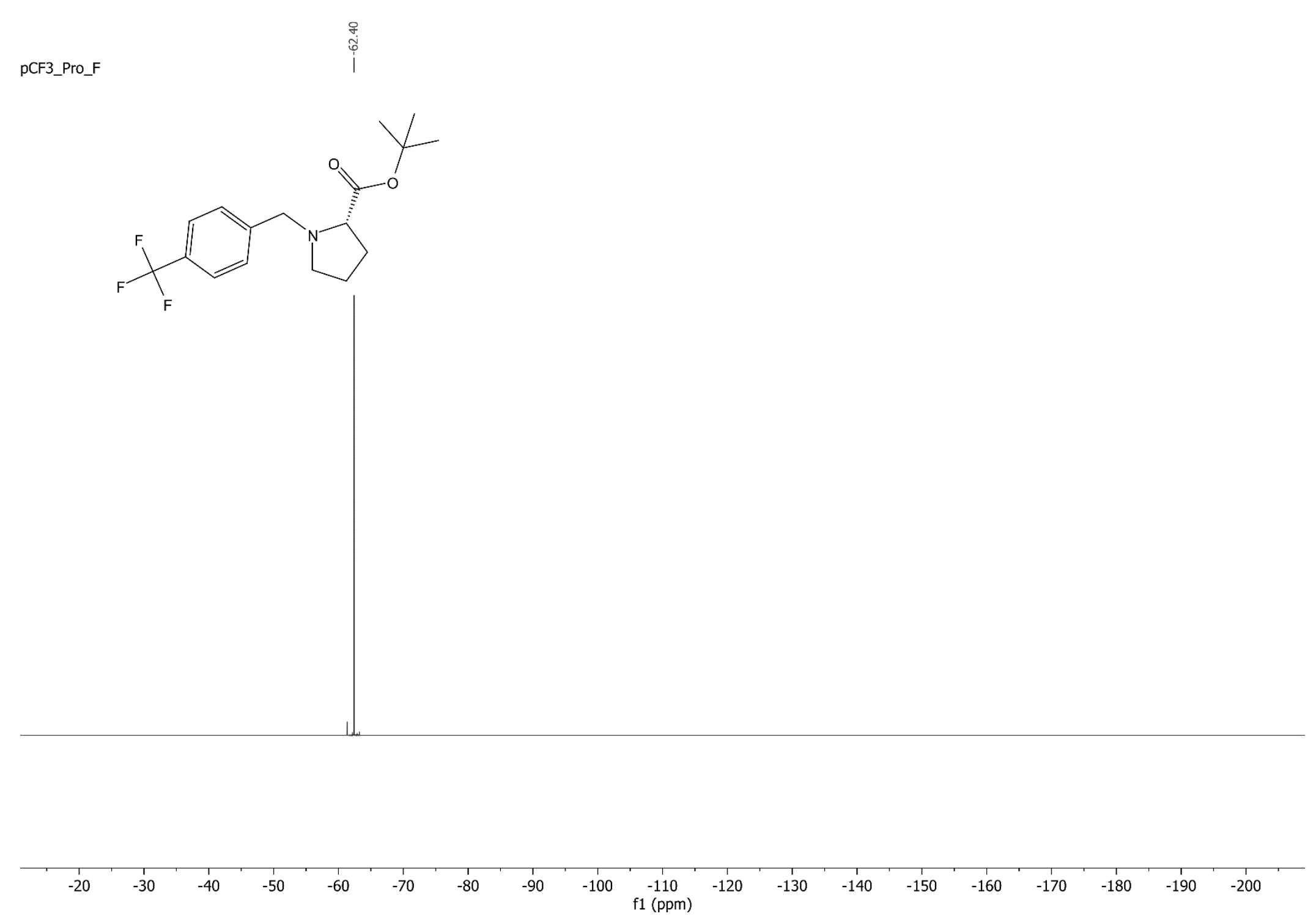


${ }^{1} \mathrm{H}$ NMR $\left(500 \mathrm{MHz}, \mathrm{CDCl}_{3}\right)$ spectrum of $\mathbf{3 c}$

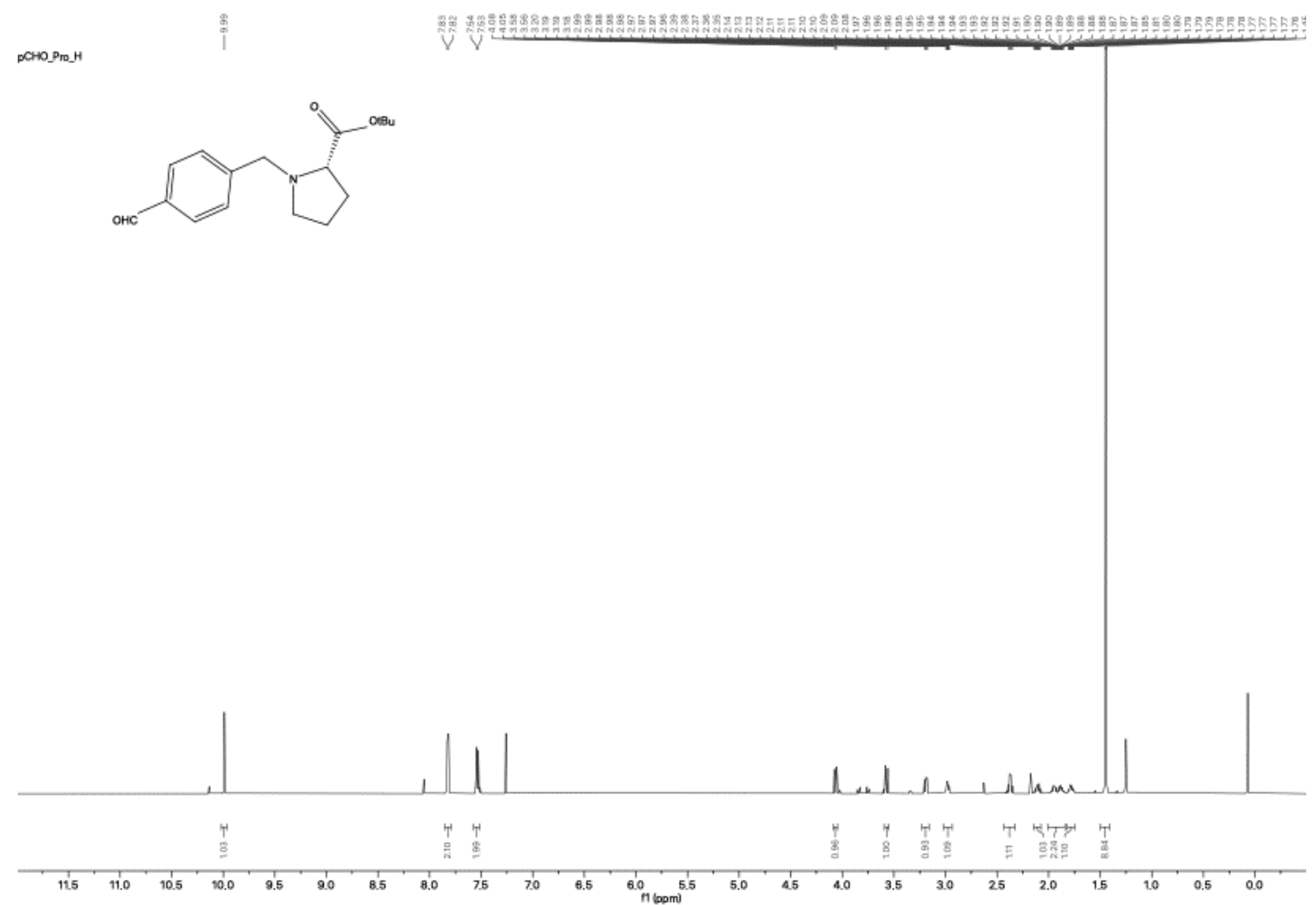


SUPPORTING INFORMATION

${ }^{13} \mathrm{C}$ NMR $\left(126 \mathrm{MHz}, \mathrm{CDCl}_{3}\right)$ spectrum of $3 \mathrm{c}$

pCHO_Pro_C
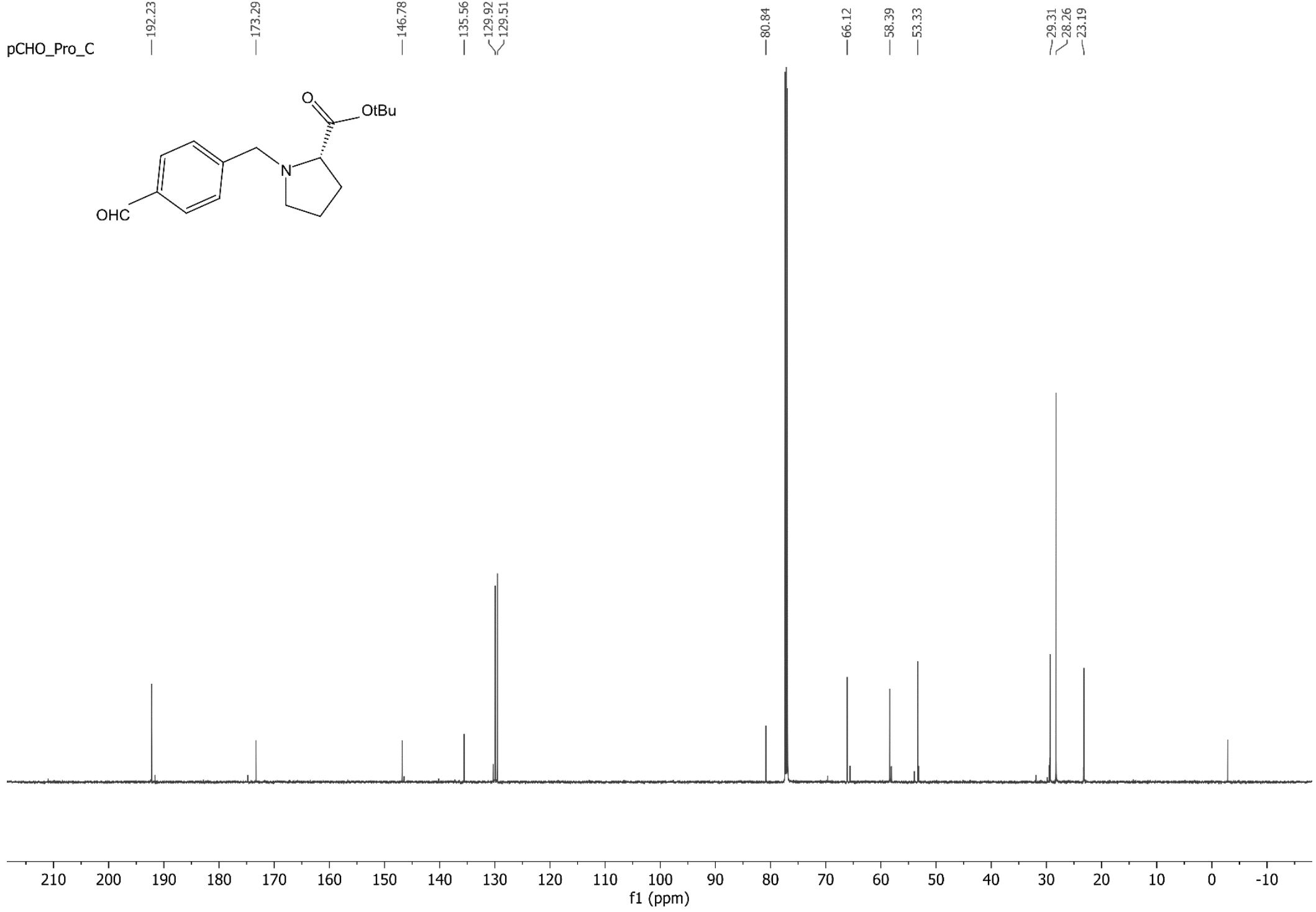

161 
${ }^{1} \mathrm{H}$ NMR $\left(400 \mathrm{MHz}, \mathrm{CDCl}_{3}\right)$ spectrum of $\mathbf{3 d}$

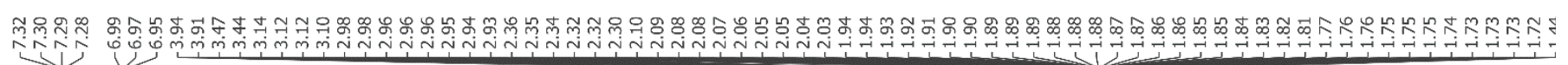

pF_Pro_H
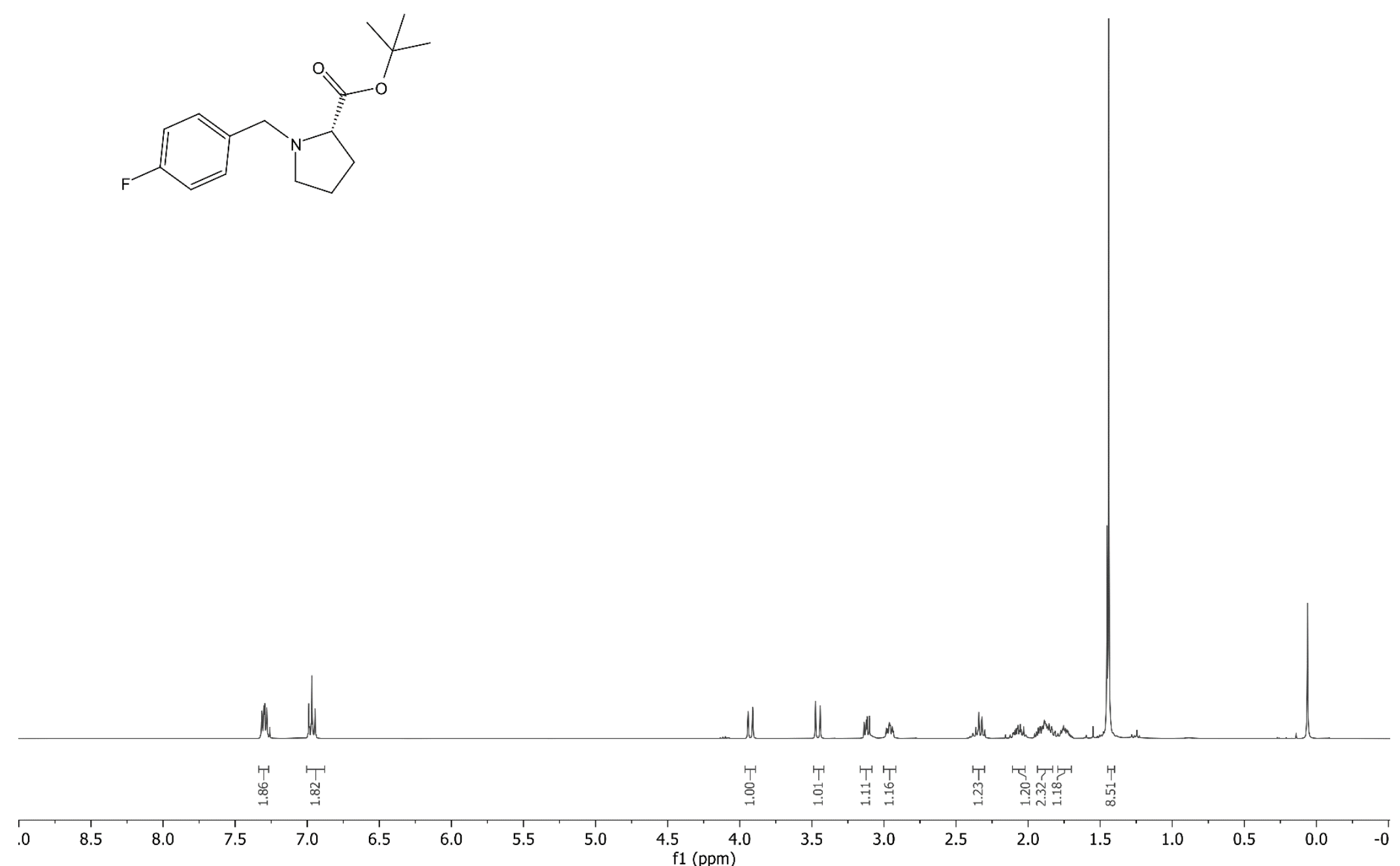
${ }^{13} \mathrm{C}$ NMR (101 MHz, $\mathrm{CDCl}_{3}$ ) spectrum of $\mathbf{3 d}$

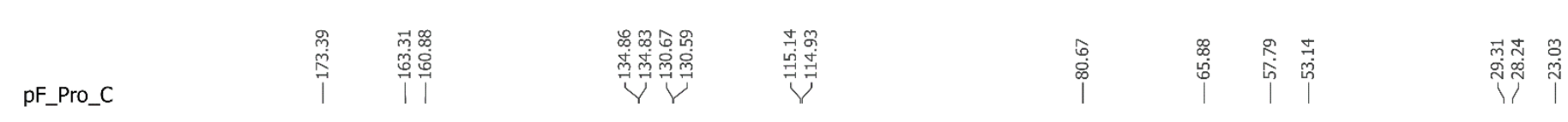
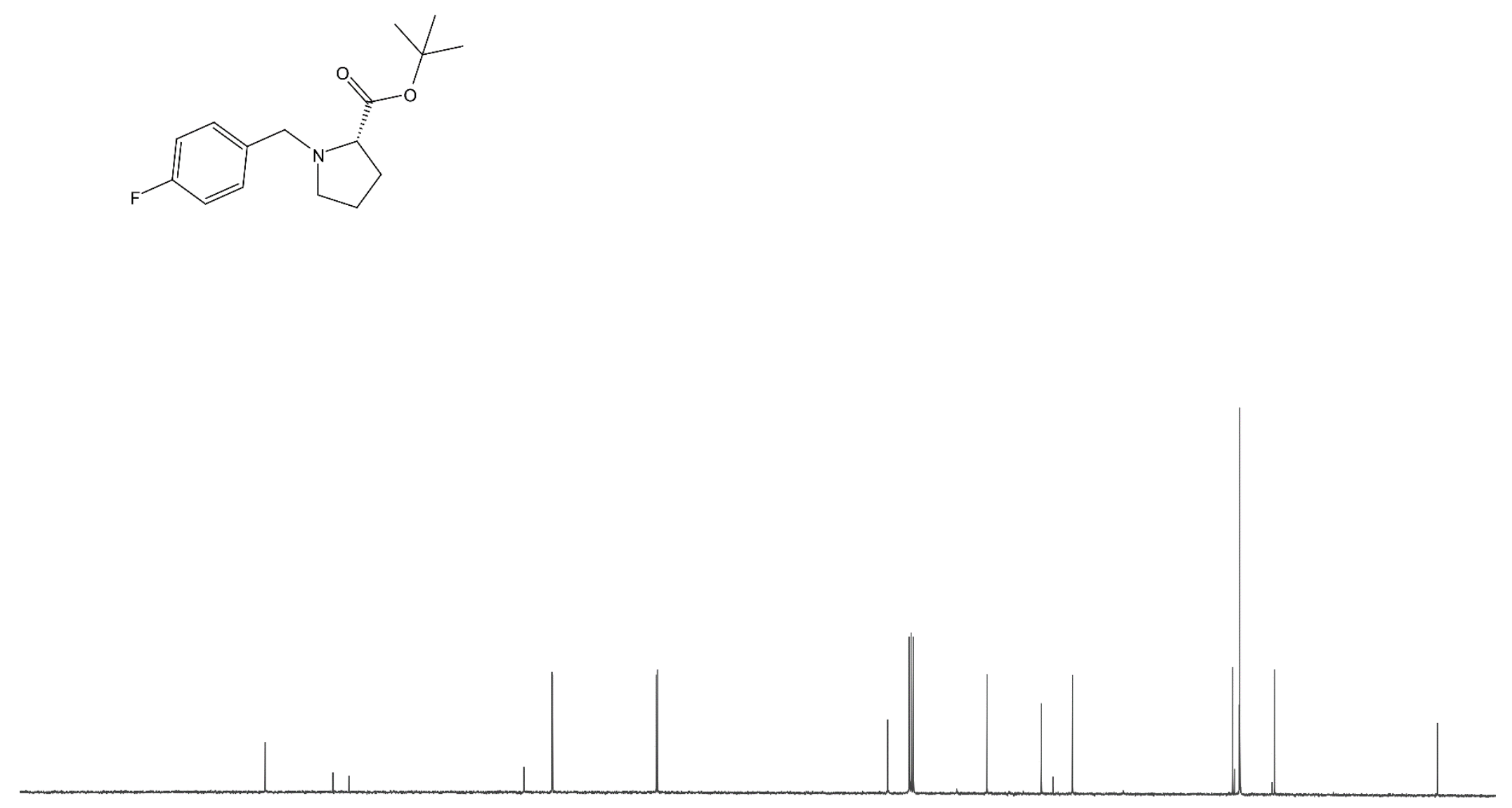

200

$180 \quad 170$

160

$140 \quad 130$

$\begin{array}{llc}120 & 110 & 100 \\ & & 100\end{array}$ 
$\left.{ }^{19} \mathrm{~F} \mathrm{NMR} \mathrm{(376} \mathrm{MHz,} \mathrm{CDCl}_{3}\right)$ spectrum of $\mathbf{3 d}$

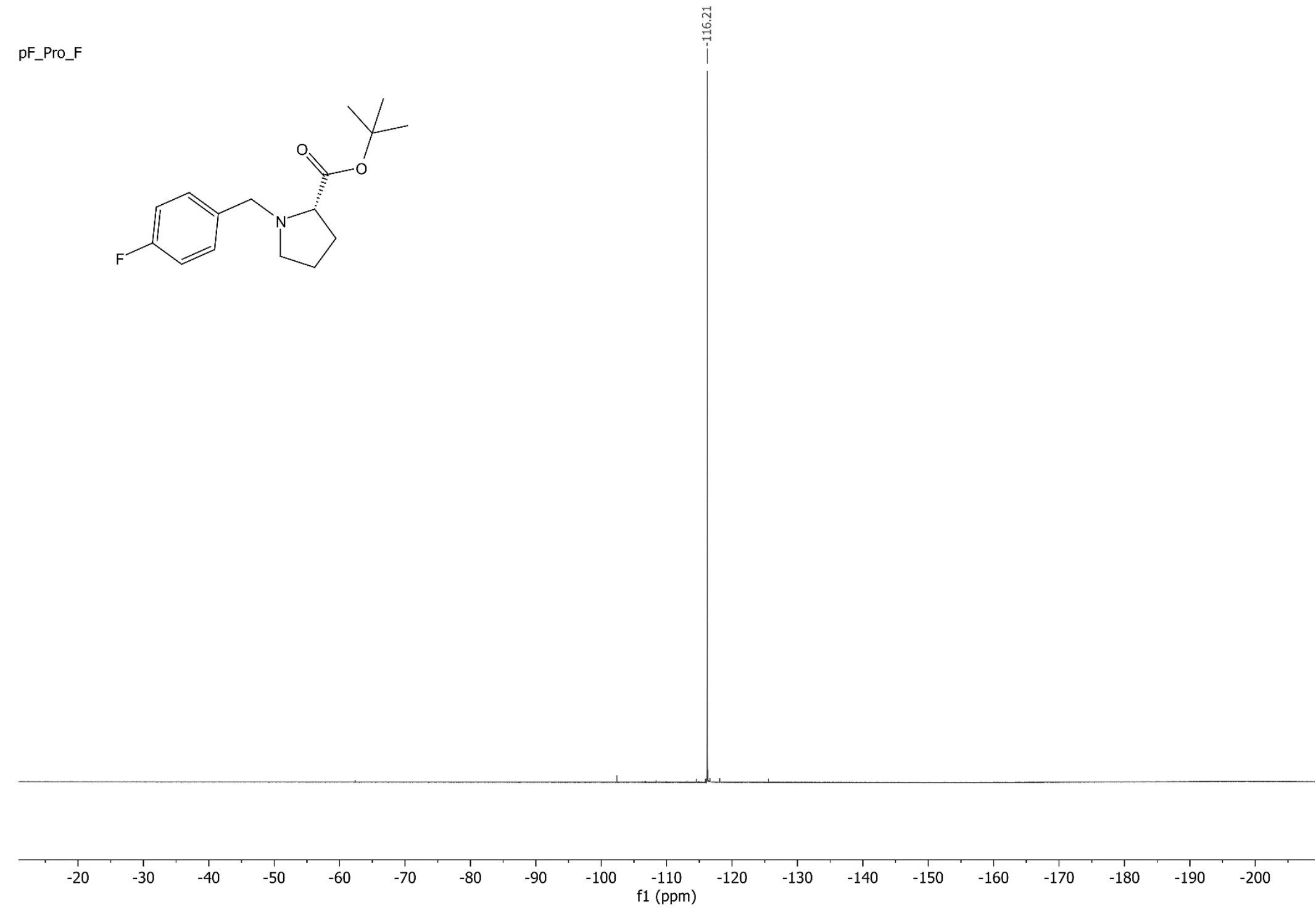


${ }^{1} \mathrm{H}$ NMR $\left(400 \mathrm{MHz}, \mathrm{CDCl}_{3}\right)$ spectrum of $\mathbf{3 e}$

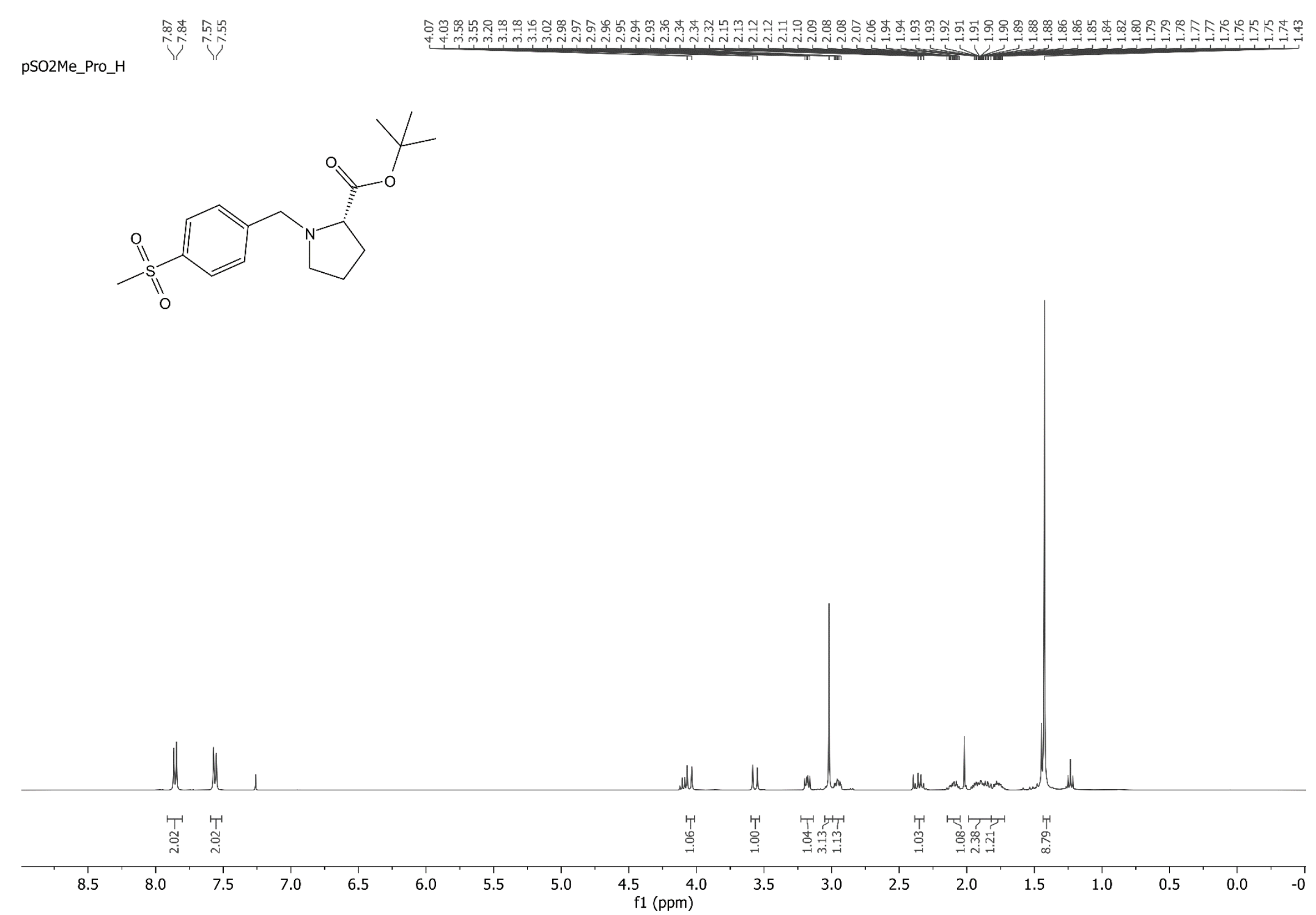


SUPPORTING INFORMATION

${ }^{13} \mathrm{C}$ NMR $\left(101 \mathrm{MHz}, \mathrm{CDCl}_{3}\right)$ spectrum of $\mathbf{3 e}$

pSO2Me_Pro_C
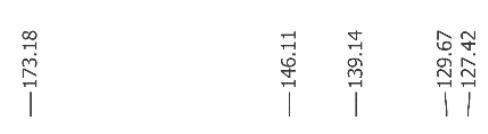

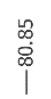

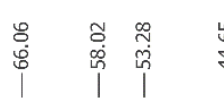

ำ
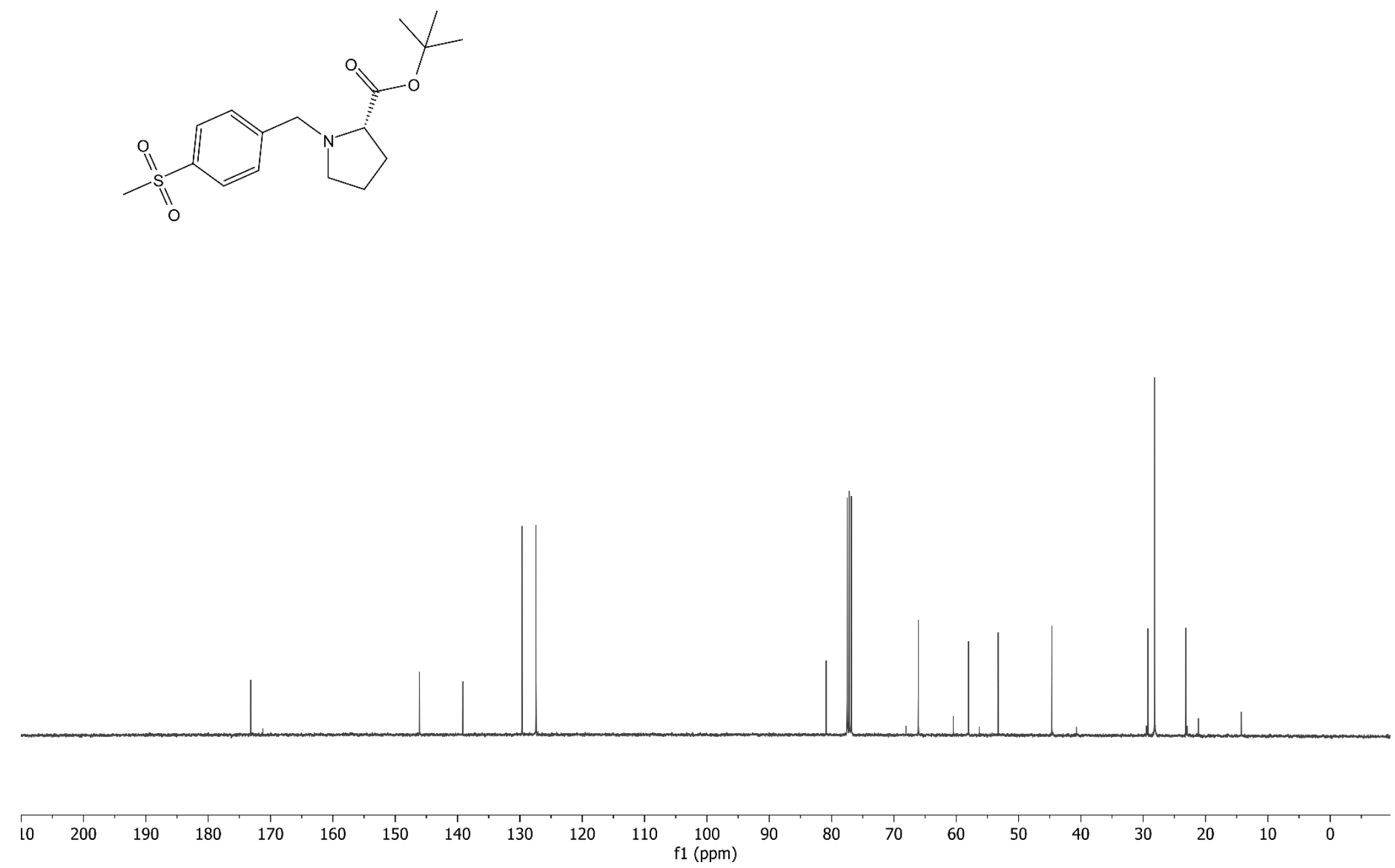

166 
${ }^{1} \mathrm{H} \mathrm{NMR}\left(400 \mathrm{MHz}, \mathrm{CDCl}_{3}\right)$ spectrum of $\mathbf{3 f}$

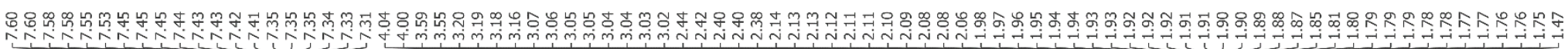
pPh_Pro_H

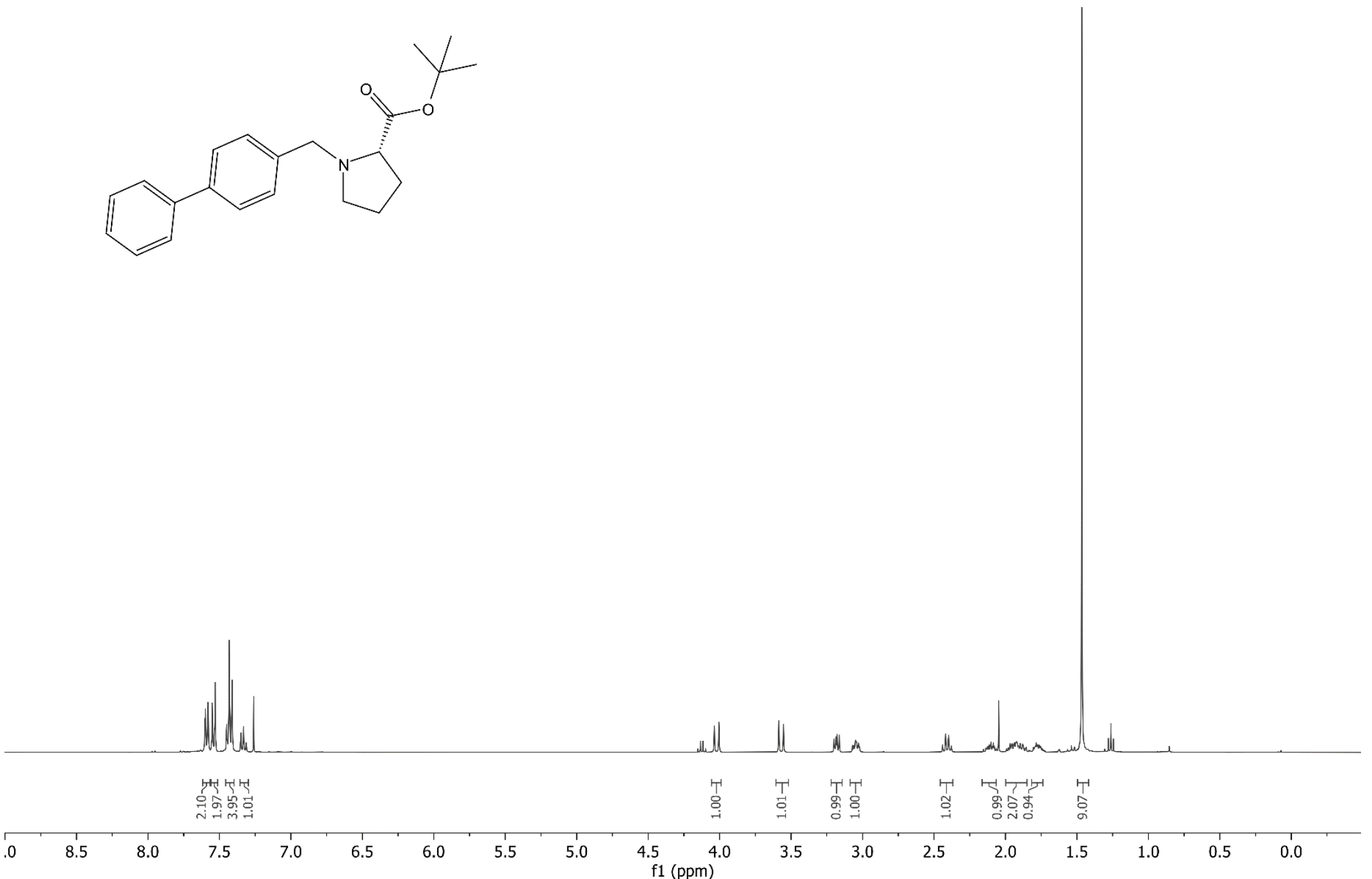


SUPPORTING INFORMATION

${ }^{13} \mathrm{C}$ NMR $\left(101 \mathrm{MHz}, \mathrm{CDCl}_{3}\right)$ spectrum of $\mathbf{3 f}$

pPh_Pro_C

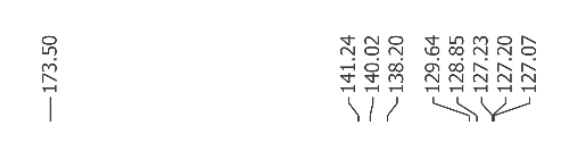

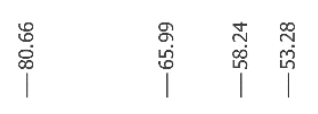

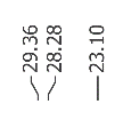
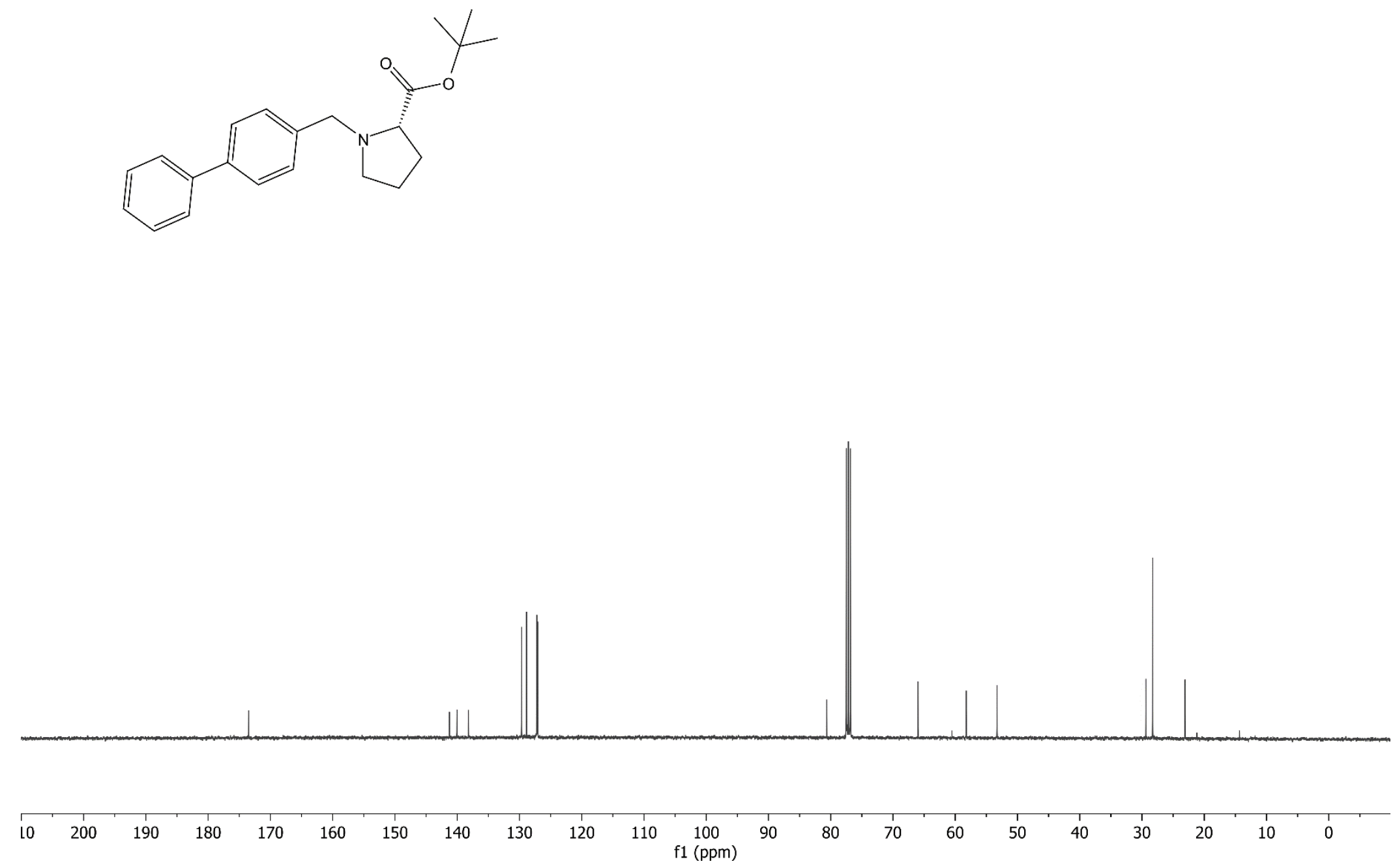

168 
${ }^{1} \mathrm{H}$ NMR $\left(600 \mathrm{MHz}, \mathrm{CDCl}_{3}\right)$ spectrum of $\mathbf{3 g}$

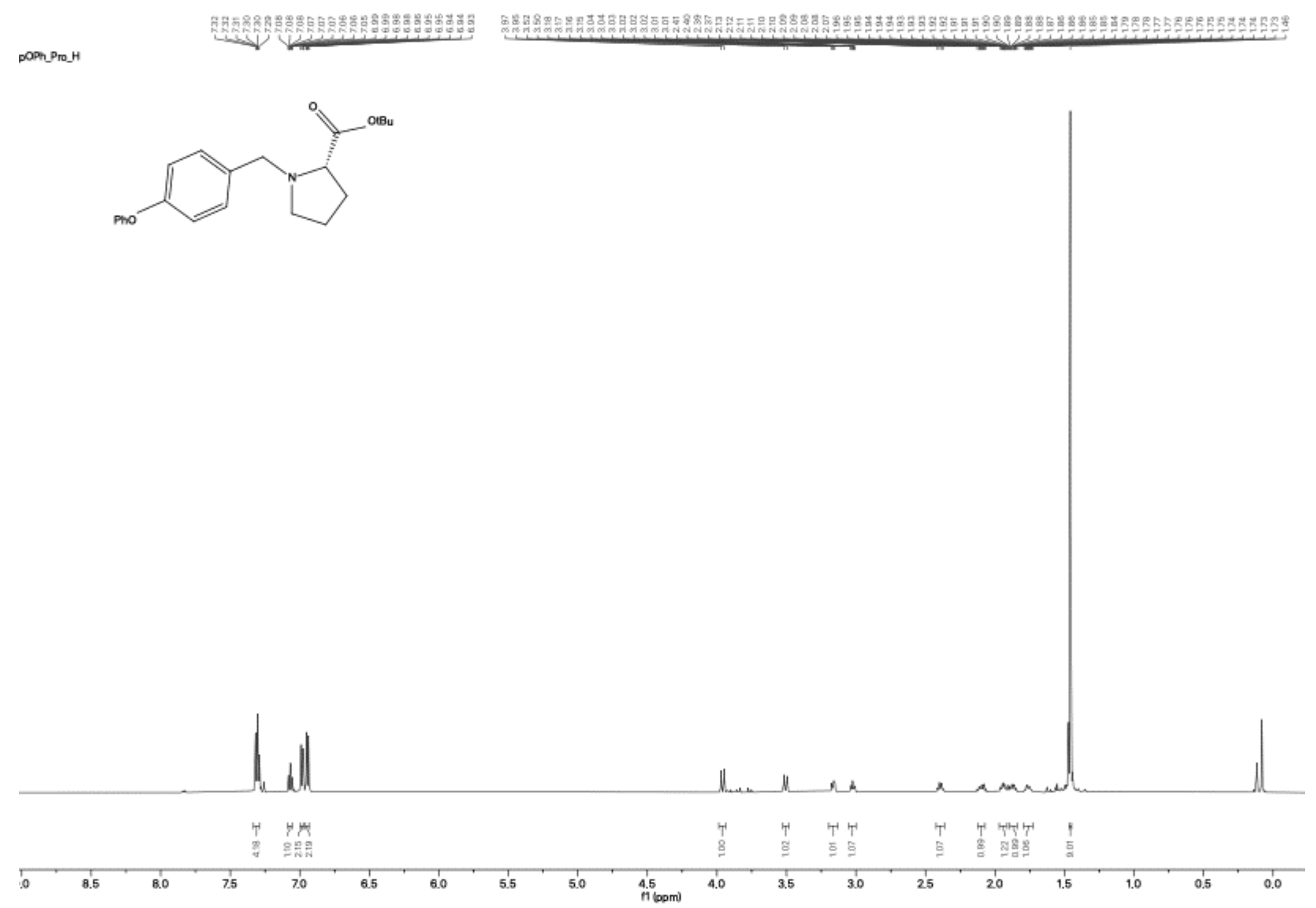


${ }^{13} \mathrm{C} \mathrm{NMR}\left(151 \mathrm{MHz}, \mathrm{CDCl}_{3}\right)$ spectrum of $\mathbf{3 g}$
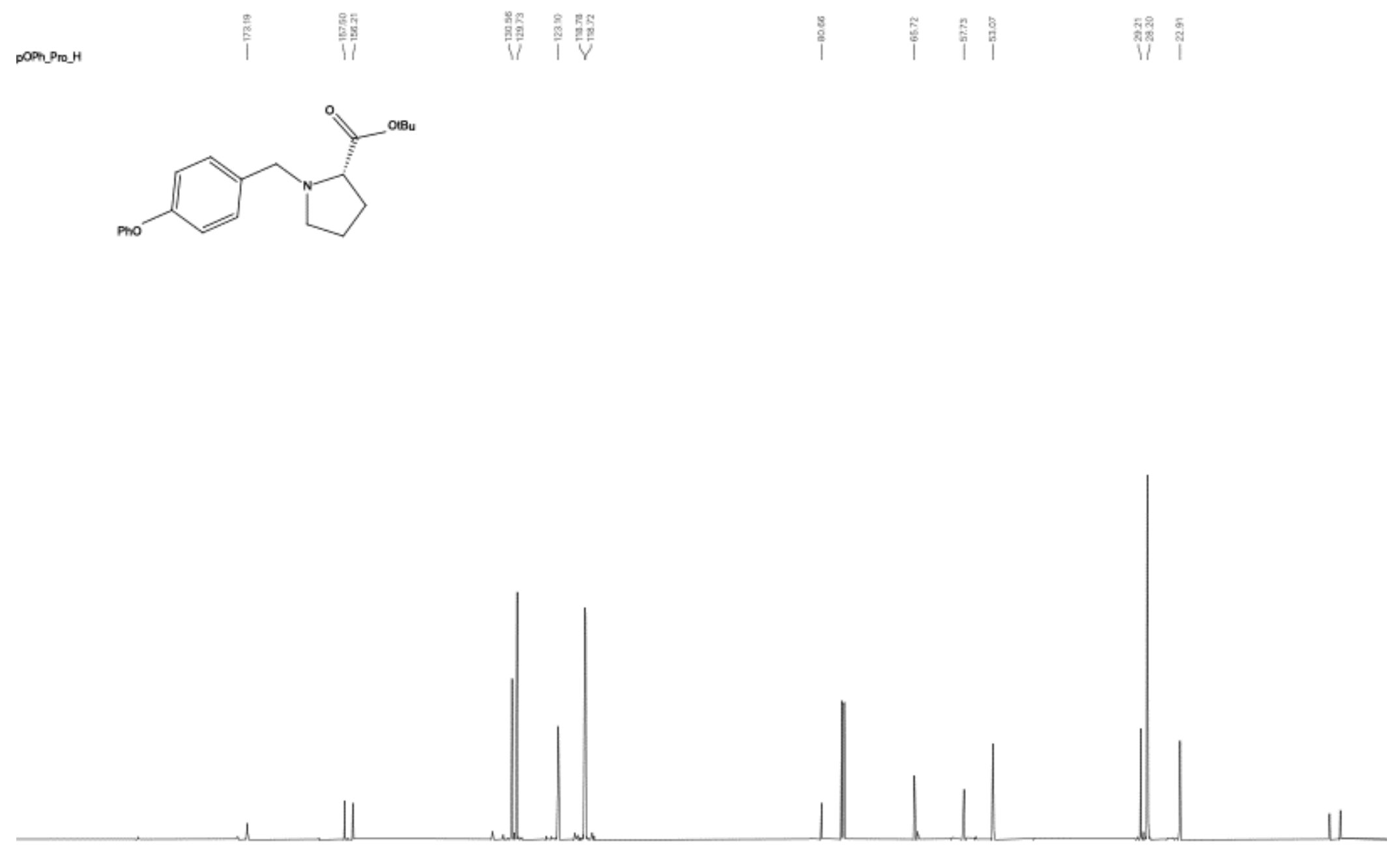

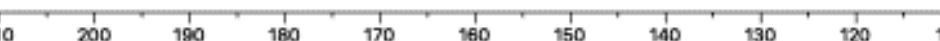

100
$1160 m$

90 
${ }^{1} \mathrm{H} \mathrm{NMR}\left(500 \mathrm{MHz}, \mathrm{CDCl}_{3}\right)$ spectrum of $\mathbf{3 h}$

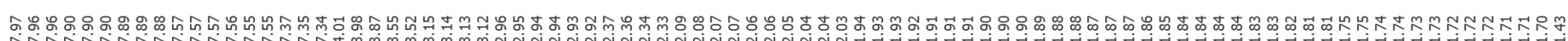
Nin mCOOMe_Pro_H<smiles>COC(=O)c1cccc(CN2CCC[C@H]2C(=O)OC(C)(C)C)c1</smiles>

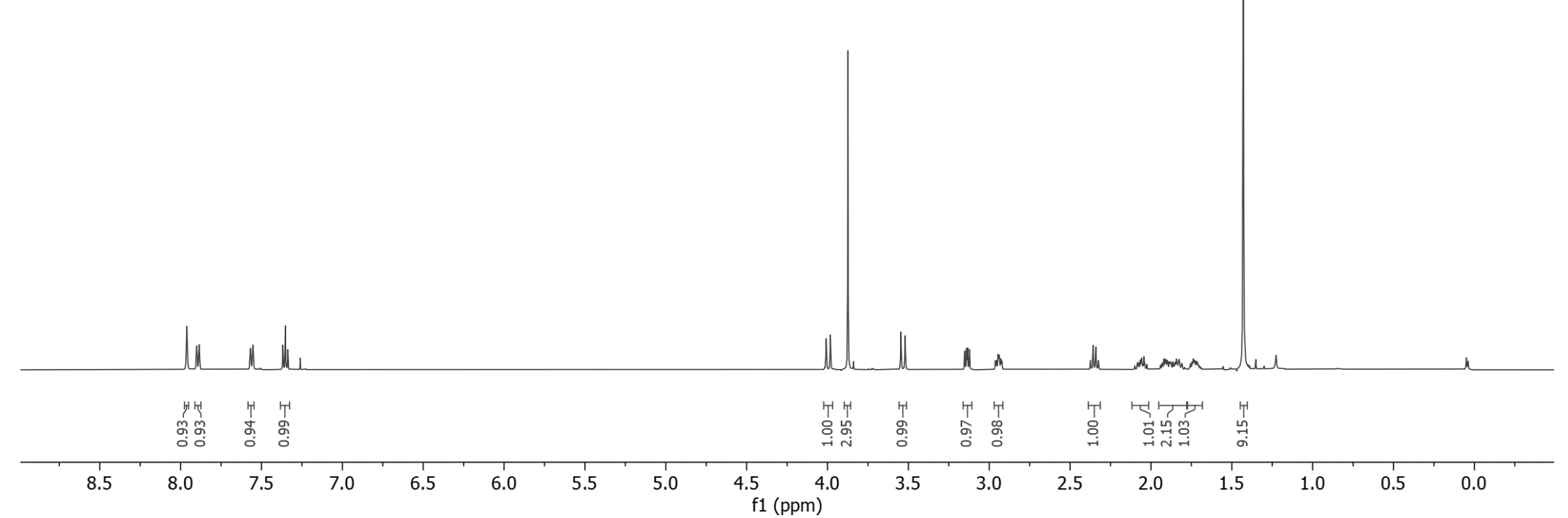


${ }^{13} \mathrm{C} \mathrm{NMR} \mathrm{(126} \mathrm{MHz,} \mathrm{CDCl}_{3}$ ) spectrum of $\mathbf{3 h}$

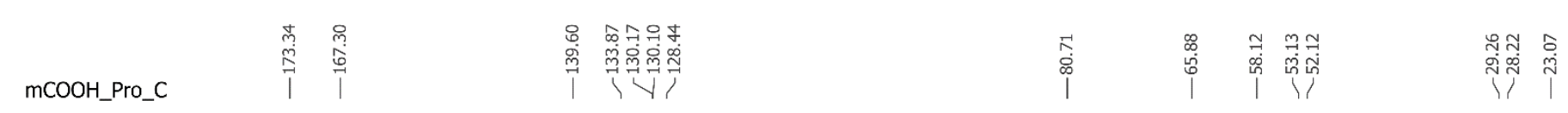
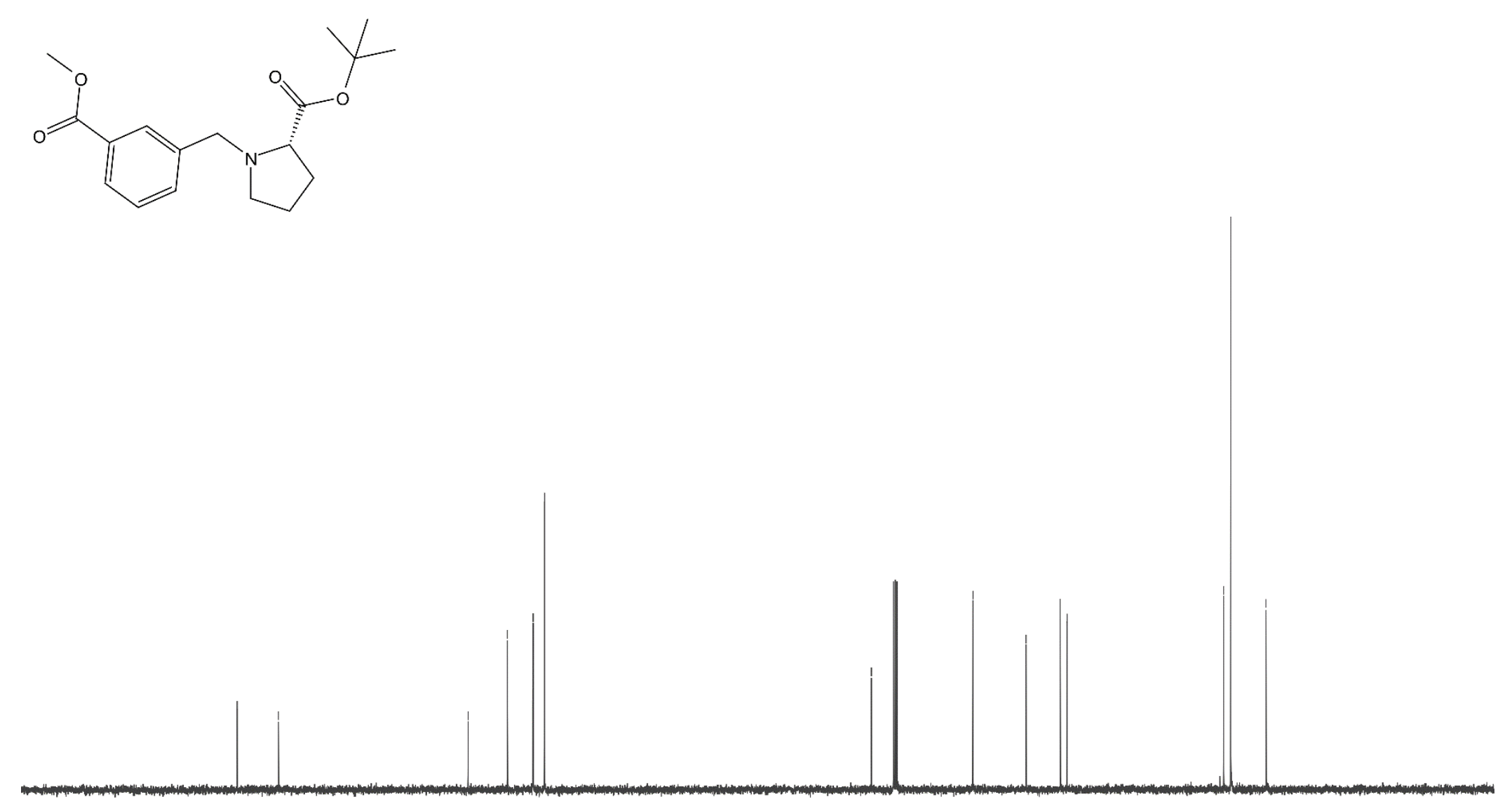

$200 \quad 190 \quad 180$

160150

$140 \quad 130 \quad 120$

$110 \quad 100 \quad 90$

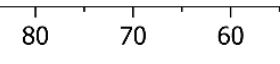

50

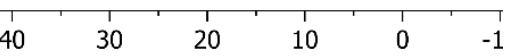


${ }^{1} \mathrm{H} \mathrm{NMR}\left(600 \mathrm{MHz}, \mathrm{CDCl}_{3}\right)$ spectrum of $\mathbf{3 i}$

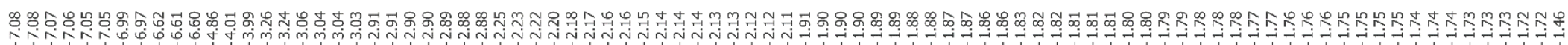
oNH2_Pro_H<smiles>CC(C)(C)OC(=O)[C@H]1CCCN1Cc1ccccc1N</smiles>

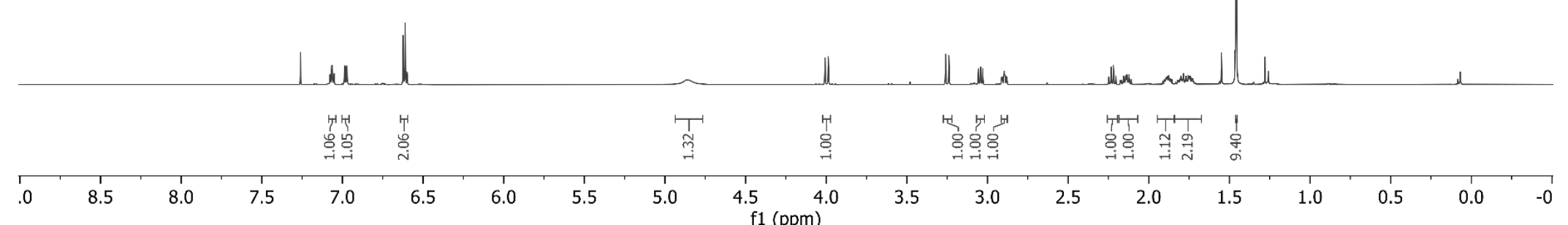


${ }^{13} \mathrm{C}$ NMR $\left(151 \mathrm{MHz}, \mathrm{CDCl}_{3}\right)$ spectrum of $\mathbf{3 i}$

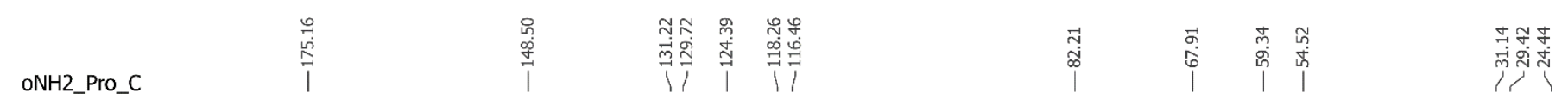
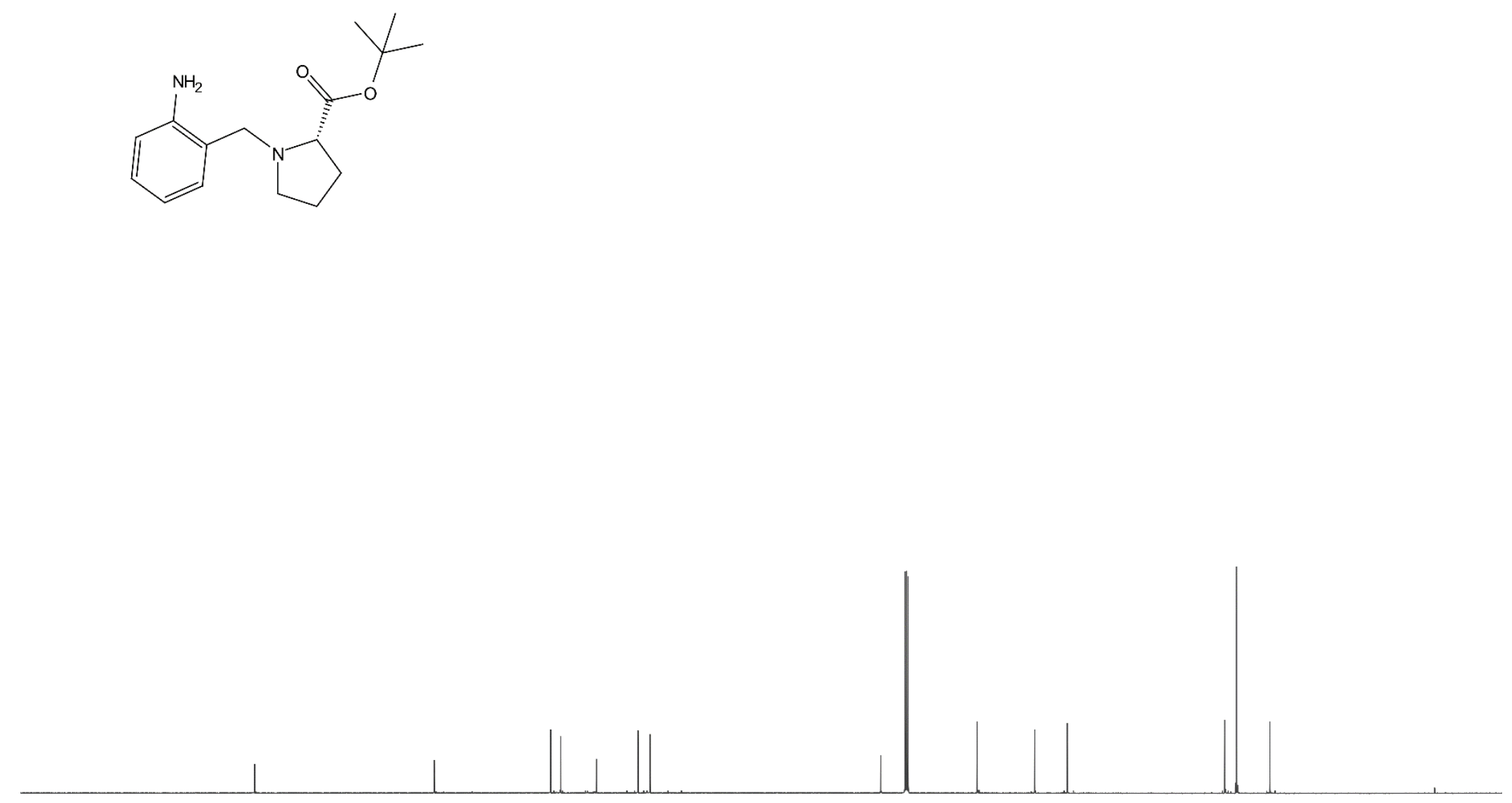

200

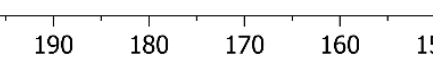

$150 \quad 140 \quad 130$

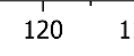

$110 \quad 100$ 
${ }^{1} \mathrm{H} \mathrm{NMR}\left(500 \mathrm{MHz}, \mathrm{CDCl}_{3}\right)$ spectrum of $\mathbf{3 j}$

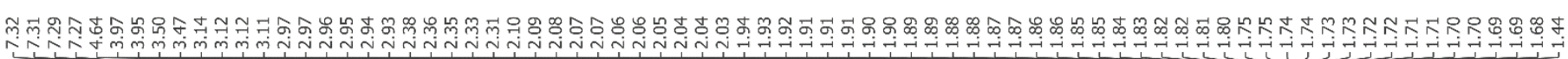

pCH2OH_Pro_H
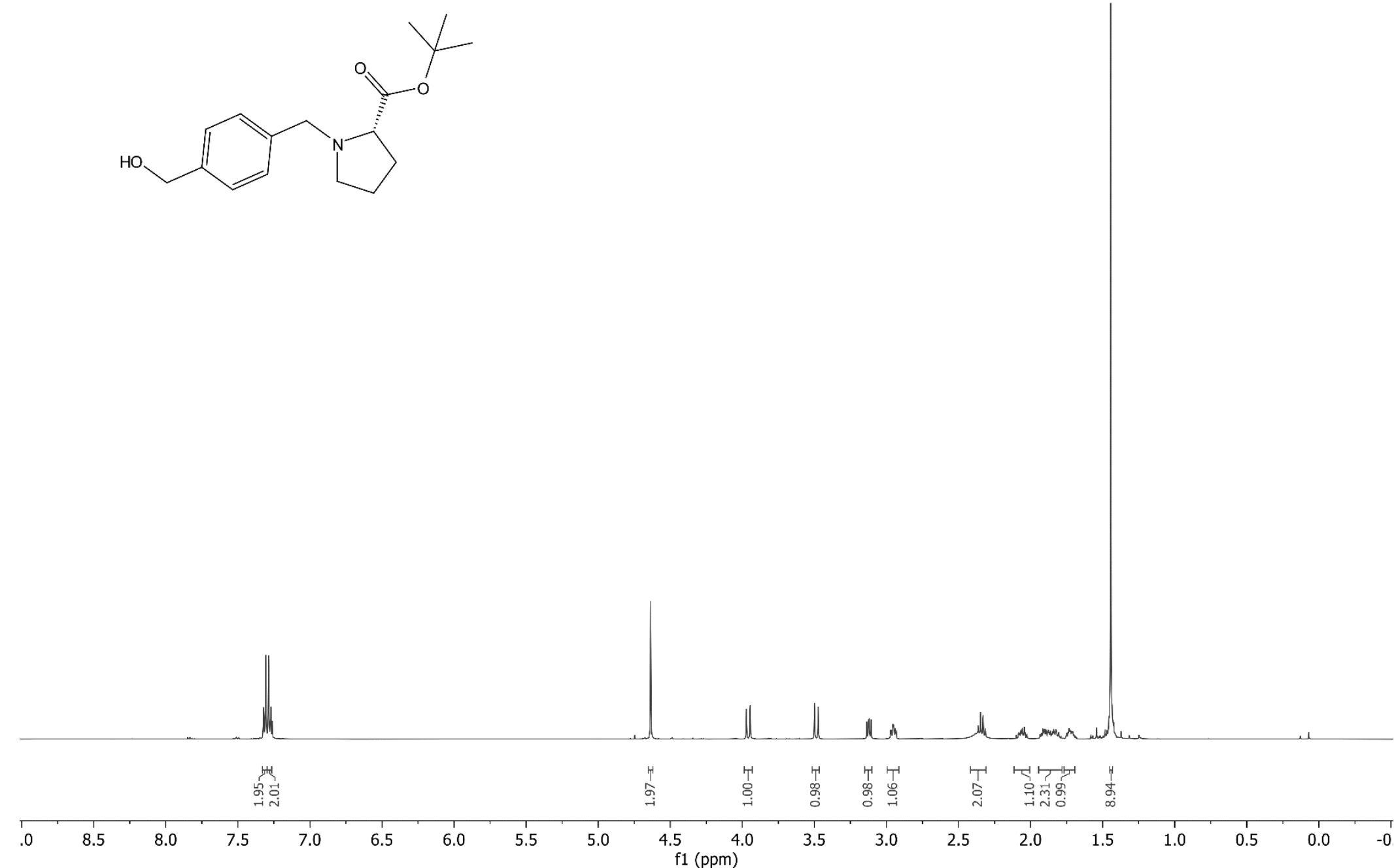
$\left.{ }^{13} \mathrm{C} \mathrm{NMR} \mathrm{(126} \mathrm{MHz,} \mathrm{CDCl}_{3}\right)$ spectrum of $\mathbf{3 j}$

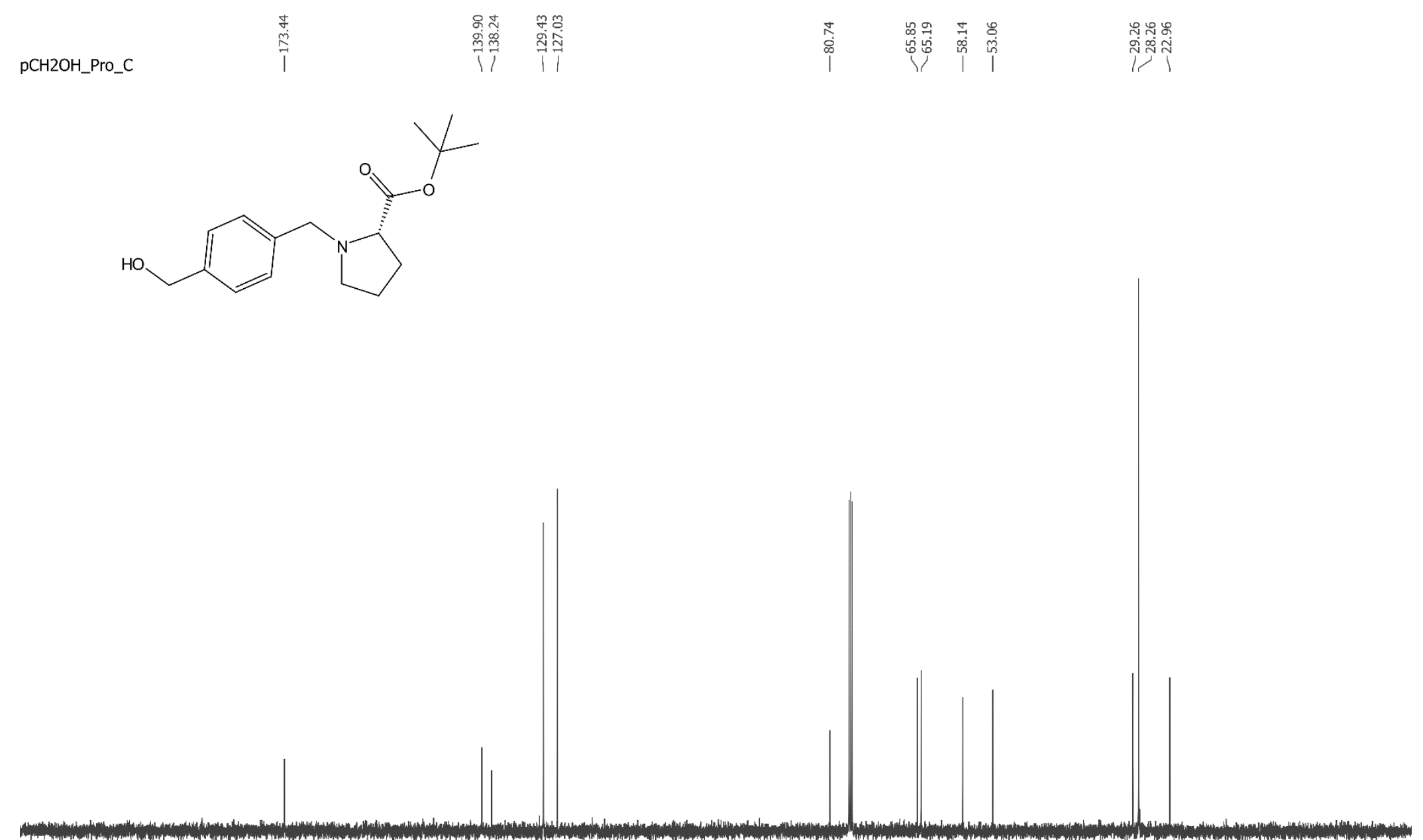

$\begin{array}{lllllll}210 & 200 & 190 & 180 & 170 & 160 & 150\end{array}$ 
${ }^{1} \mathrm{H}$ NMR $\left(500 \mathrm{MHz}, \mathrm{CDCl}_{3}\right)$ spectrum of $\mathbf{3 k}$

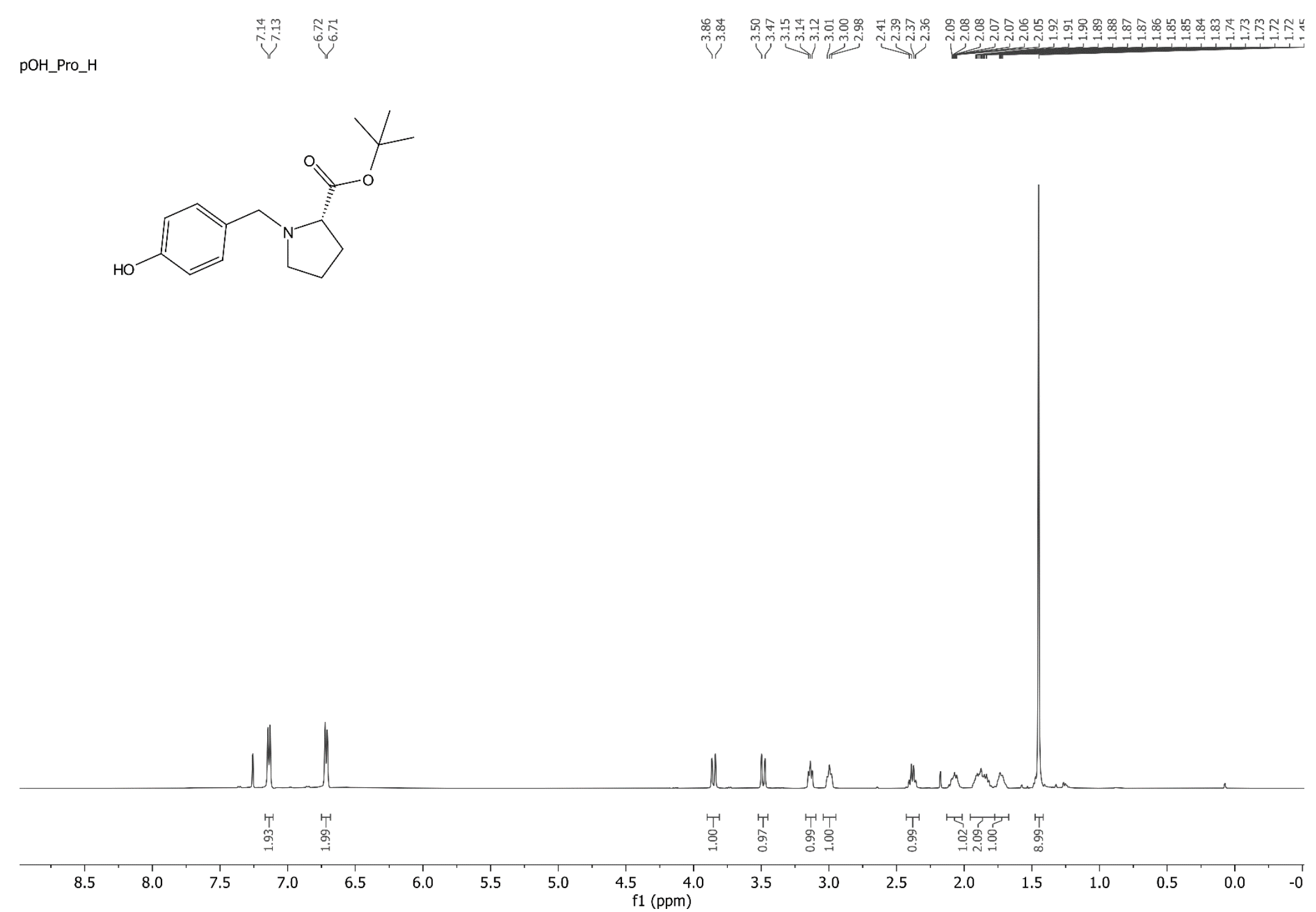


${ }^{13} \mathrm{C}$ NMR $\left(126 \mathrm{MHz}, \mathrm{CDCl}_{3}\right)$ spectrum of $\mathbf{3 k}$

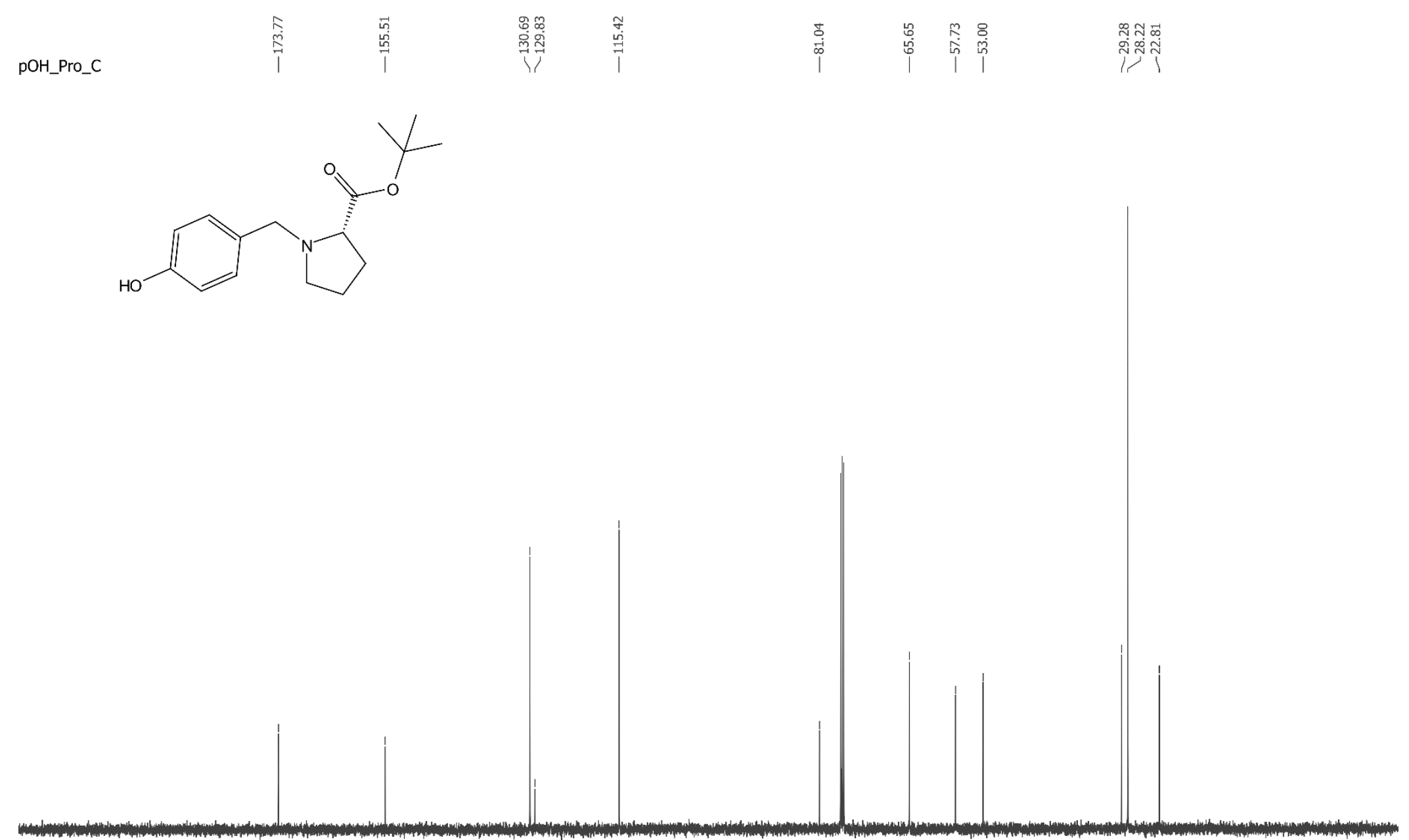

$\begin{array}{llllllll}210 & 200 & 190 & 180 & 170 & 160 & 150 & 140\end{array}$

$130 \quad 120 \quad 110 \quad 100$

$\begin{array}{lll}1 & 1 & 1 \\ 80 & 70 & 60\end{array}$

50

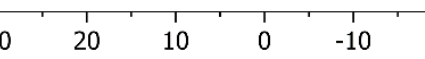


${ }^{1} \mathrm{H} \mathrm{NMR}\left(400 \mathrm{MHz}, \mathrm{CDCl}_{3}\right)$ spectrum of $\mathbf{3 l}$

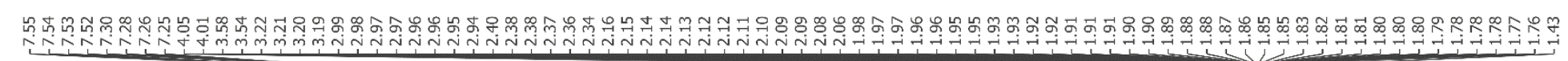

pCN_mF_Pro_H
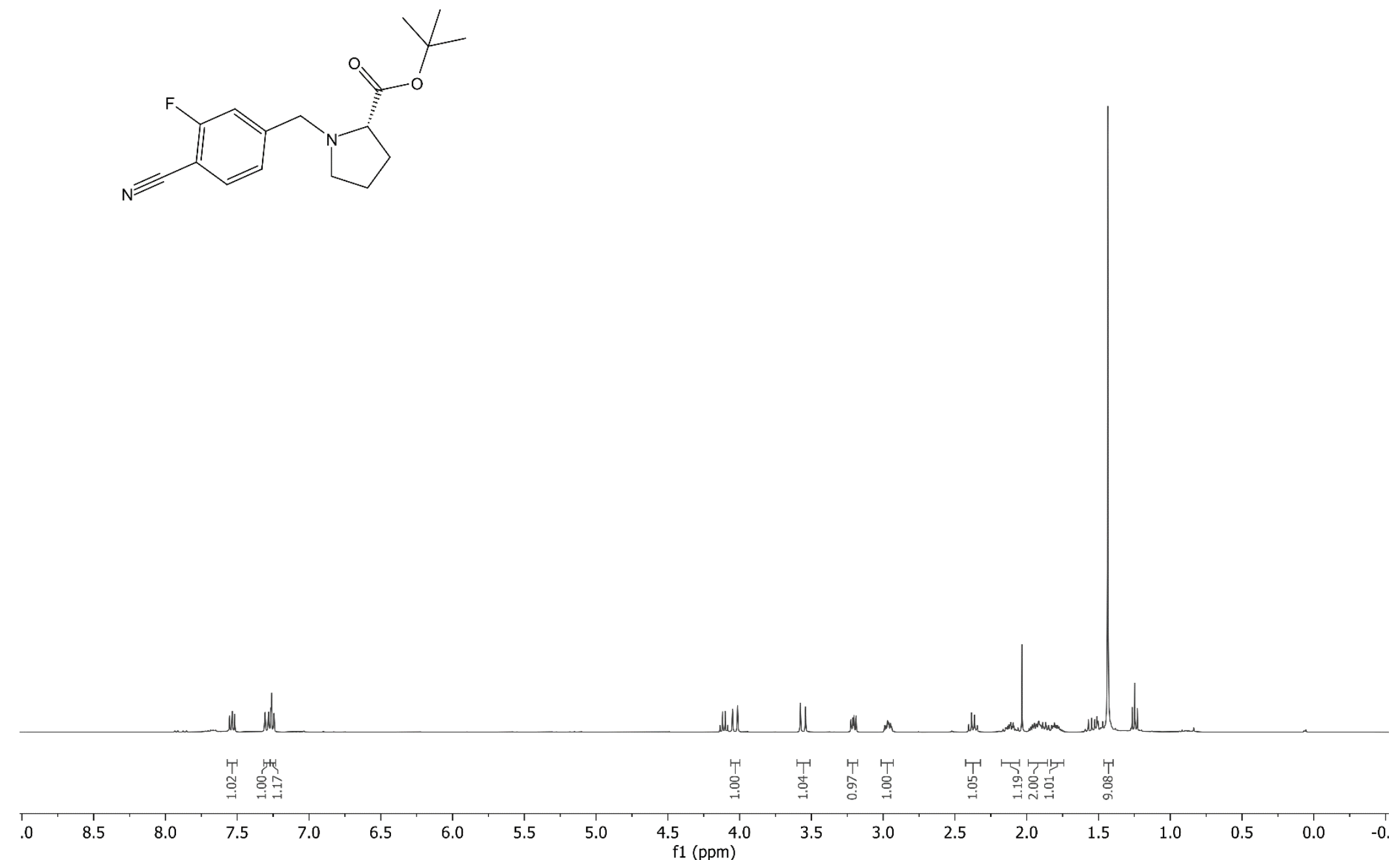
SUPPORTING INFORMATION

${ }^{13} \mathrm{C}$ NMR (101 MHz, CDCl $)$ spectrum of 3I

pCN_mF_Pro_C
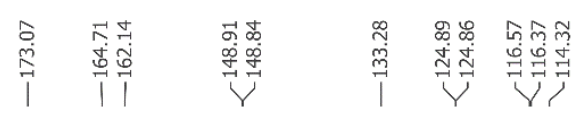

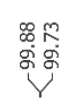

$\underset{\substack{0 \\ 0}}{\substack{0 \\ \mid}}$

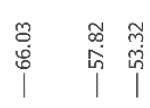

ํำ
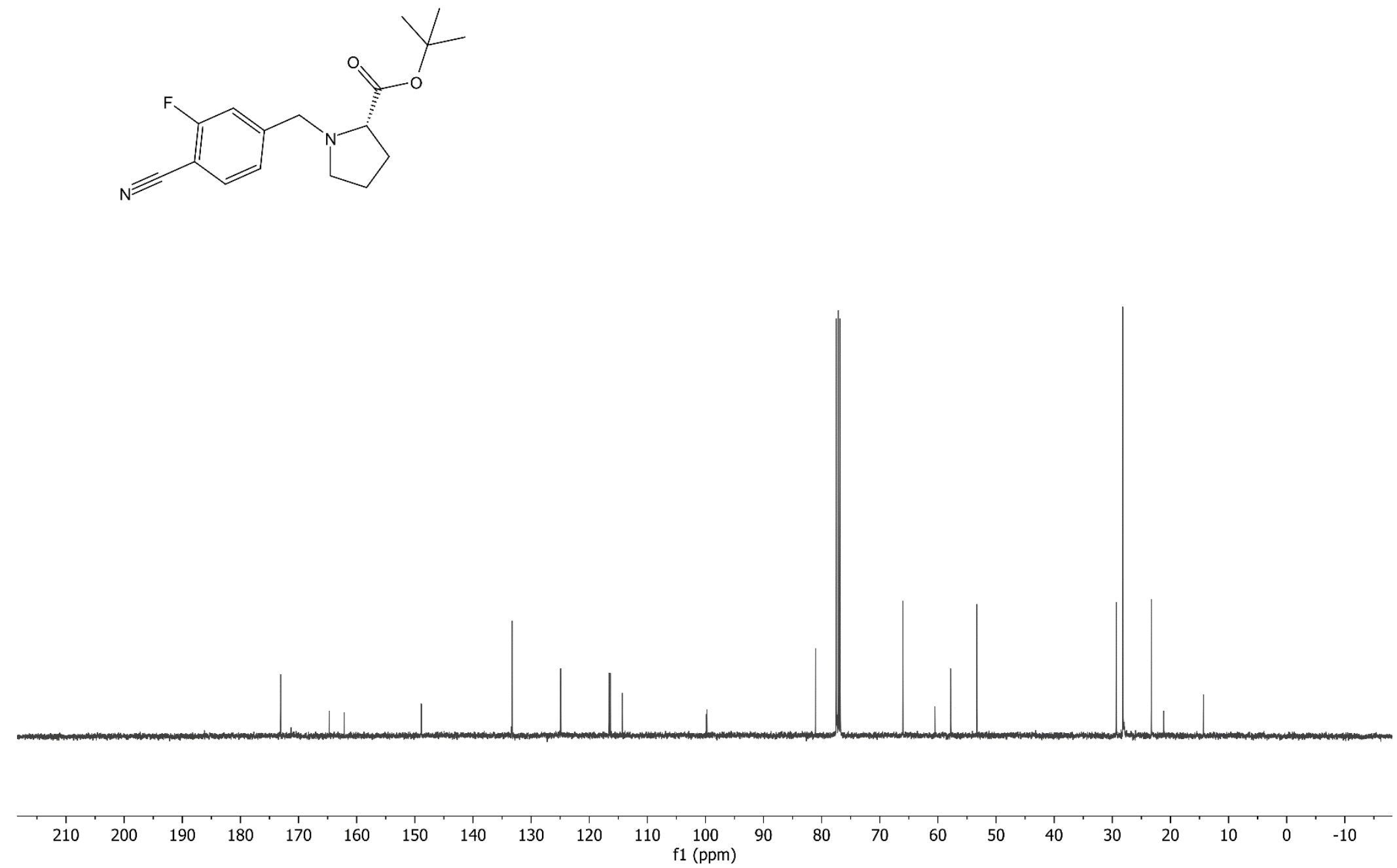

180 
${ }^{19} \mathrm{~F} \mathrm{NMR} \mathrm{(376} \mathrm{MHz,} \mathrm{CDCl}_{3}$ ) spectrum of $\mathbf{3 l}$

pCN_mF_Pro_F<smiles>Cc1ccc(CC2CCCC2C(=O)OC(C)(C)C)cc1I</smiles>

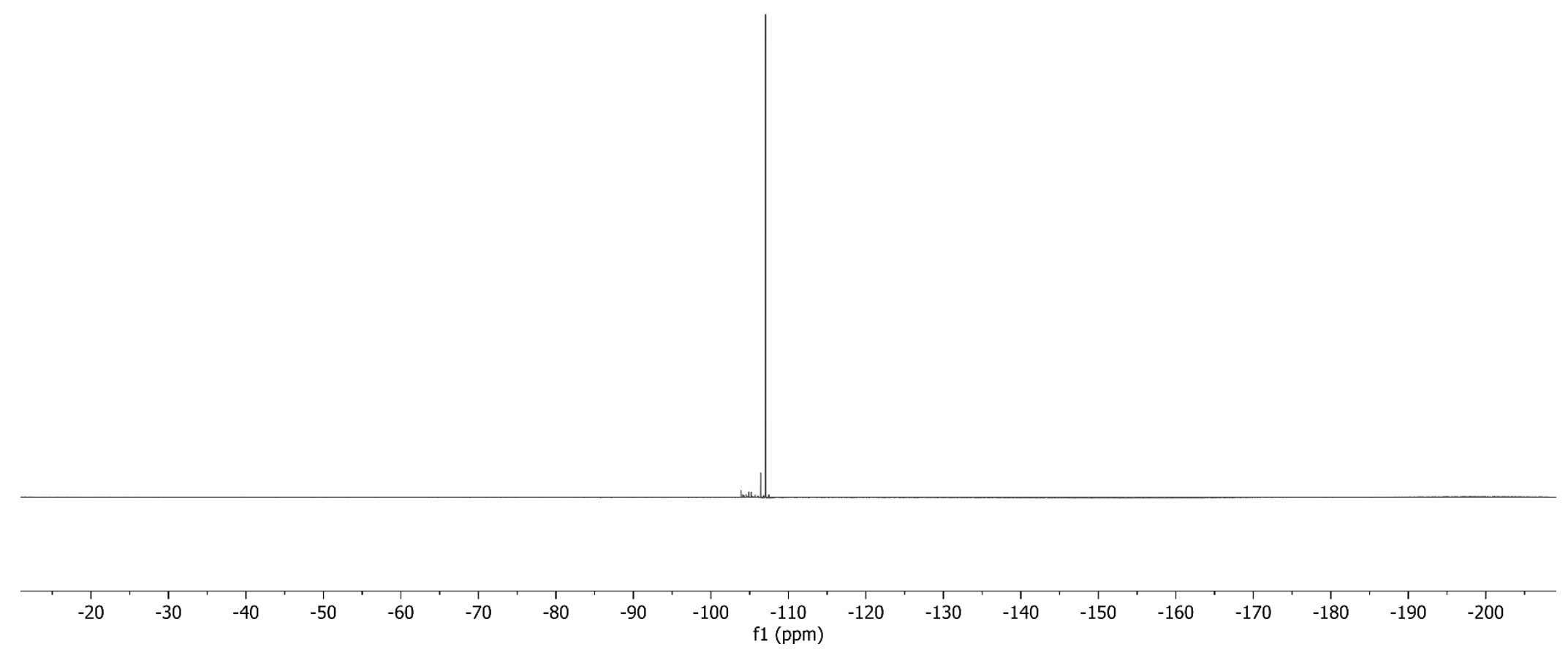


${ }^{1} \mathrm{H} \mathrm{NMR}\left(400 \mathrm{MHz}, \mathrm{CDCl}_{3}\right)$ spectrum of $3 \mathrm{~m}$

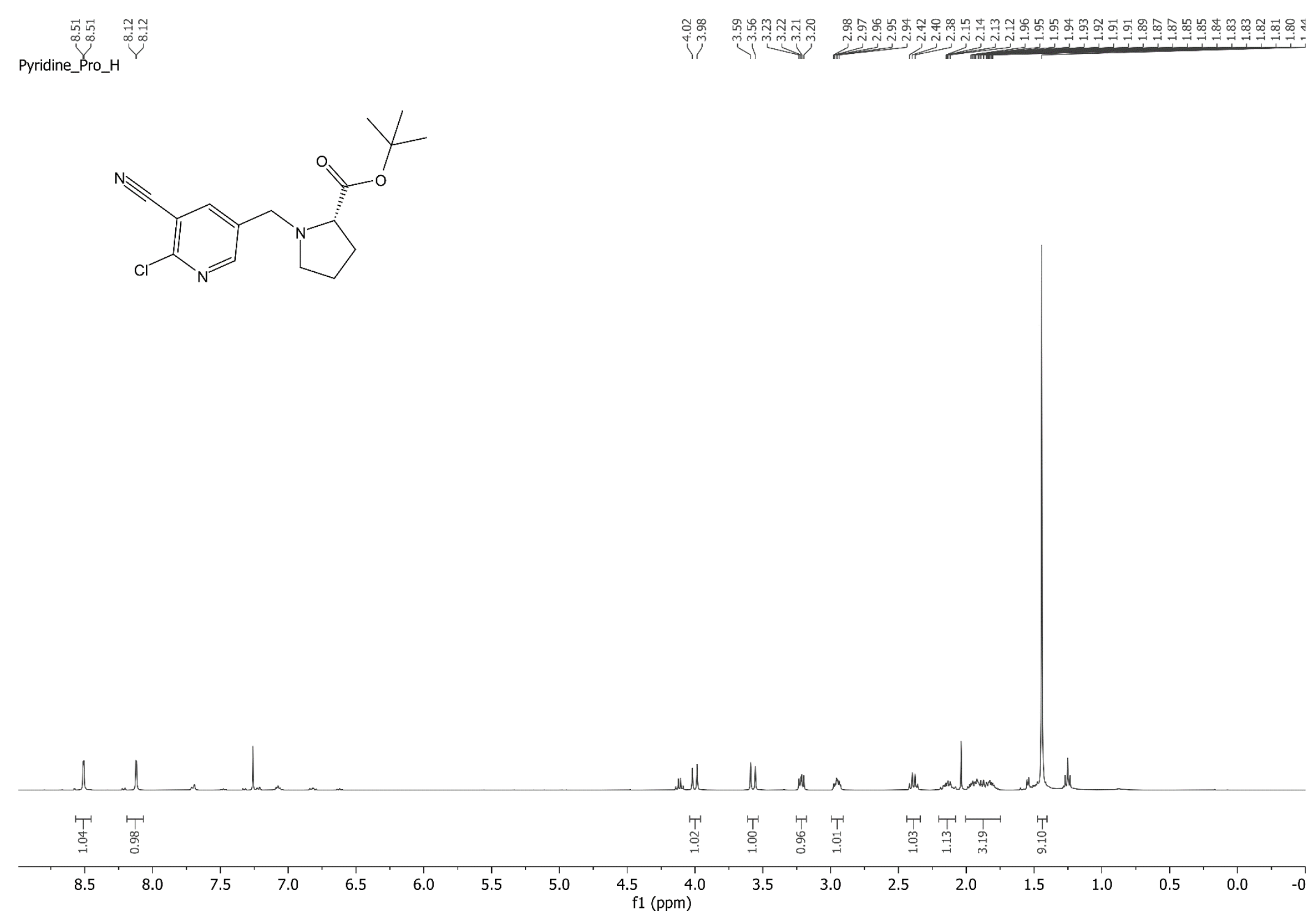


SUPPORTING INFORMATION

${ }^{13} \mathrm{C}$ NMR $(101 \mathrm{MHz}, \mathrm{CDCl})_{3}$ ) spectrum of $3 \mathrm{~m}$

Pyridine_Pro_C

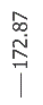

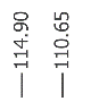

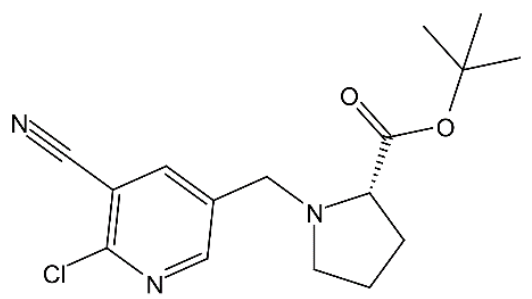

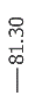

น

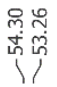

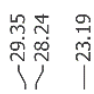

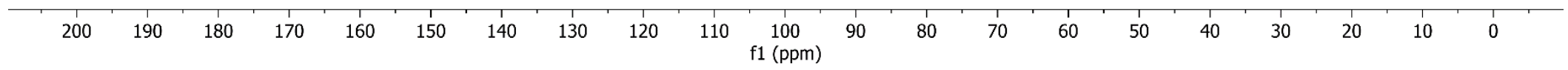

183 
${ }^{13} \mathrm{C} \mathrm{NMR}\left(400 \mathrm{MHz}, \mathrm{CDCl}_{3}\right)$ spectrum of $3 \mathrm{n}$

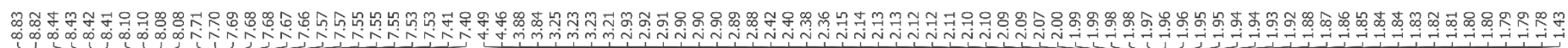
Quinoline_Pro_H
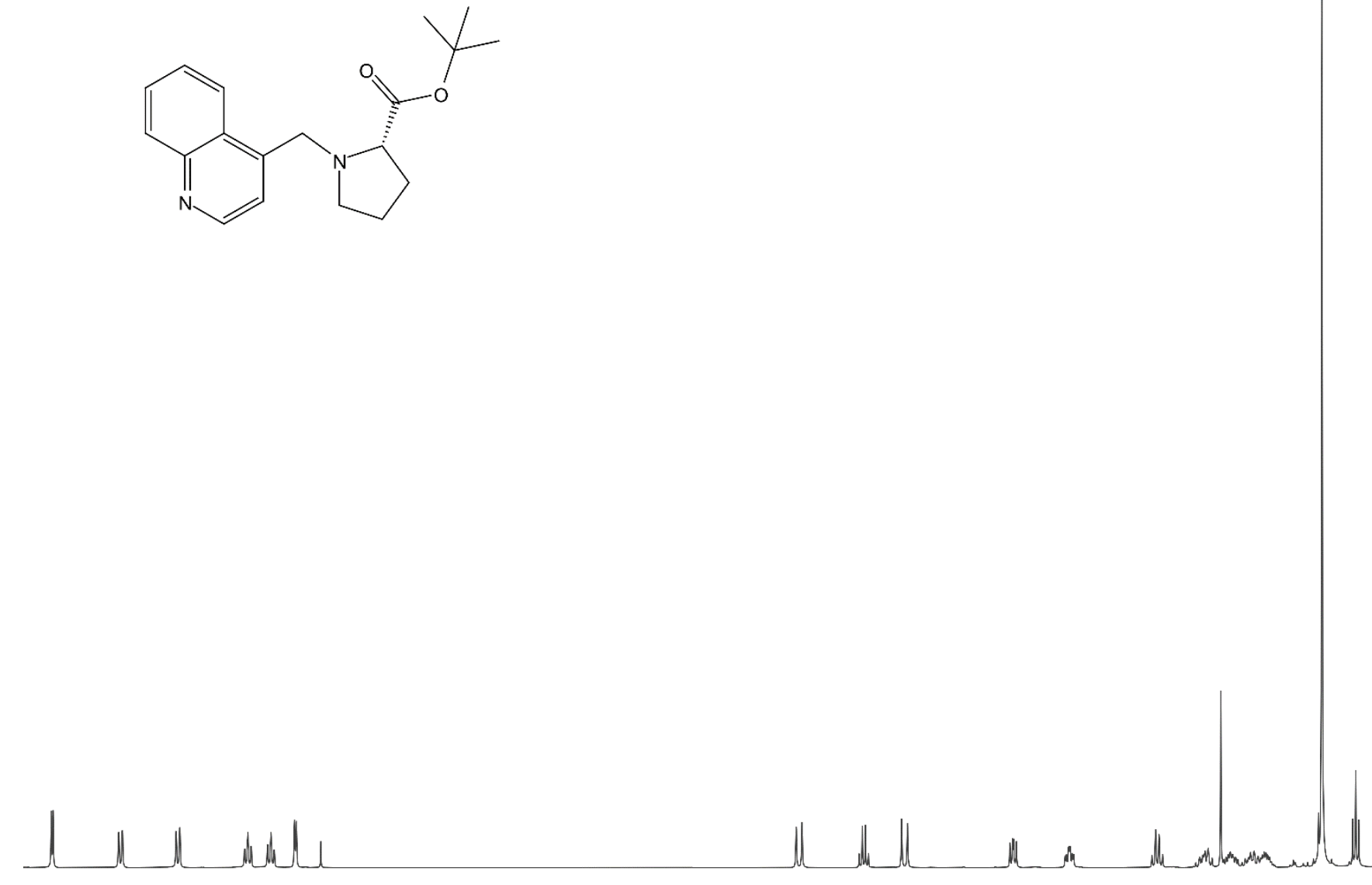

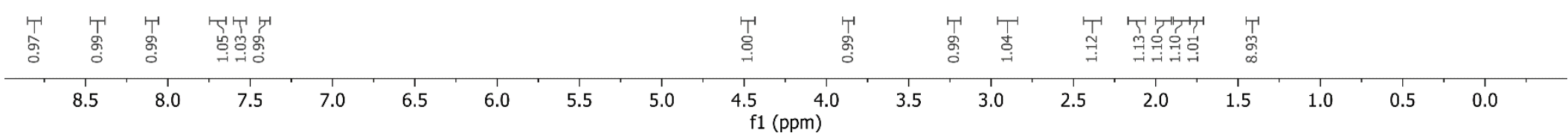


SUPPORTING INFORMATION

${ }^{13} \mathrm{C}$ NMR $\left(101 \mathrm{MHz}, \mathrm{CDCl}_{3}\right)$ spectrum of $\mathbf{3 n}$

Quinoline_Pro_C
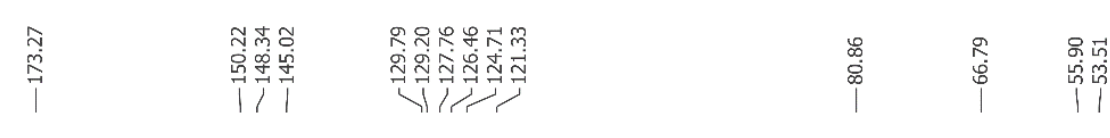

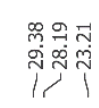
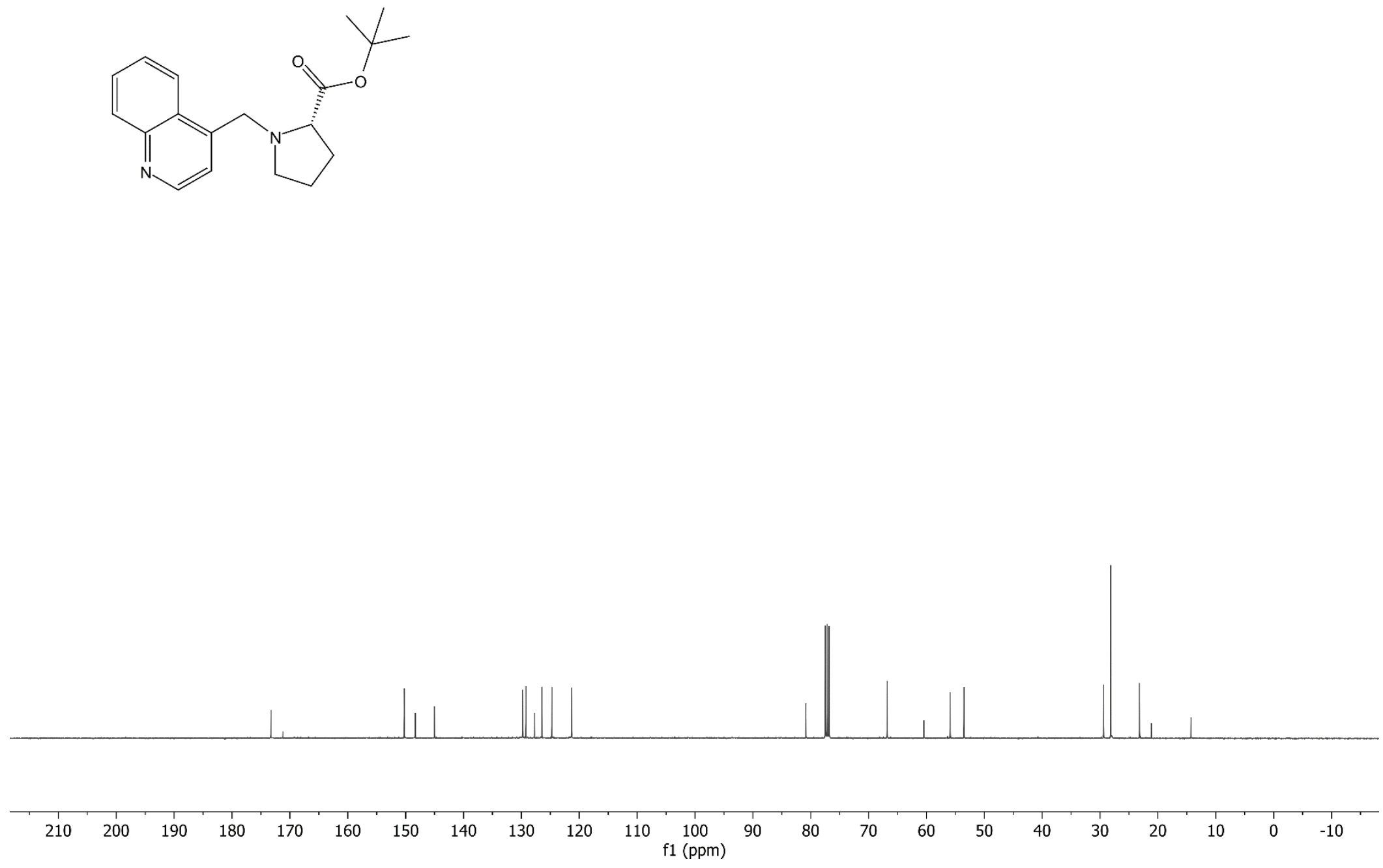

185 
${ }^{1} \mathrm{H} \mathrm{NMR}\left(400 \mathrm{MHz}, \mathrm{CDCl}_{3}\right)$ spectrum of 30

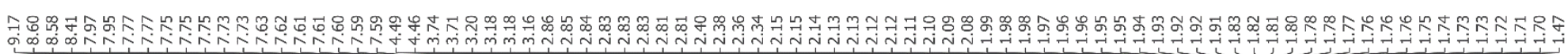

Isoquinoline_Pro_H
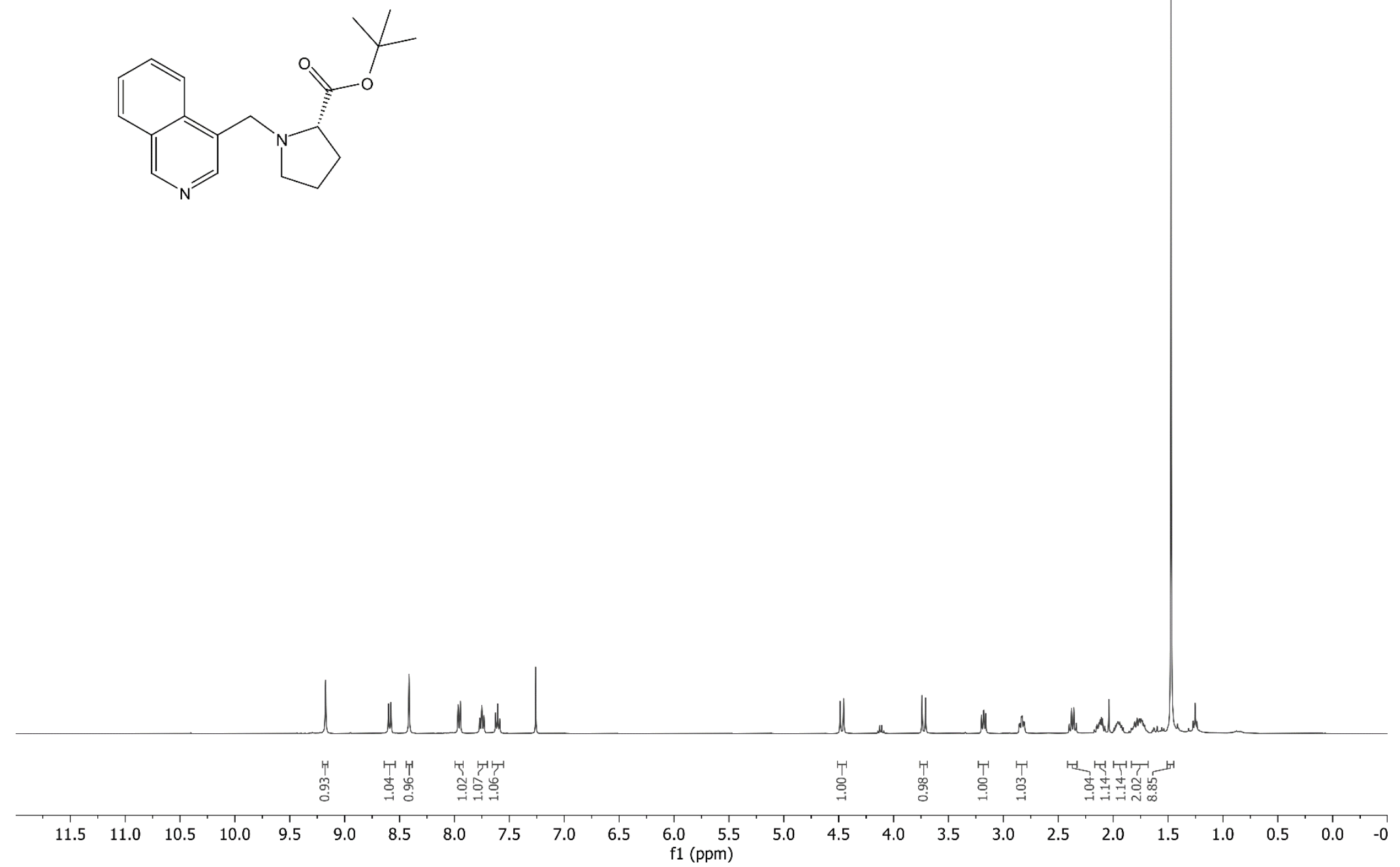
SUPPORTING INFORMATION

${ }^{13} \mathrm{C}$ NMR $\left(101 \mathrm{MHz}, \mathrm{CDCl}_{3}\right)$ spectrum of 30

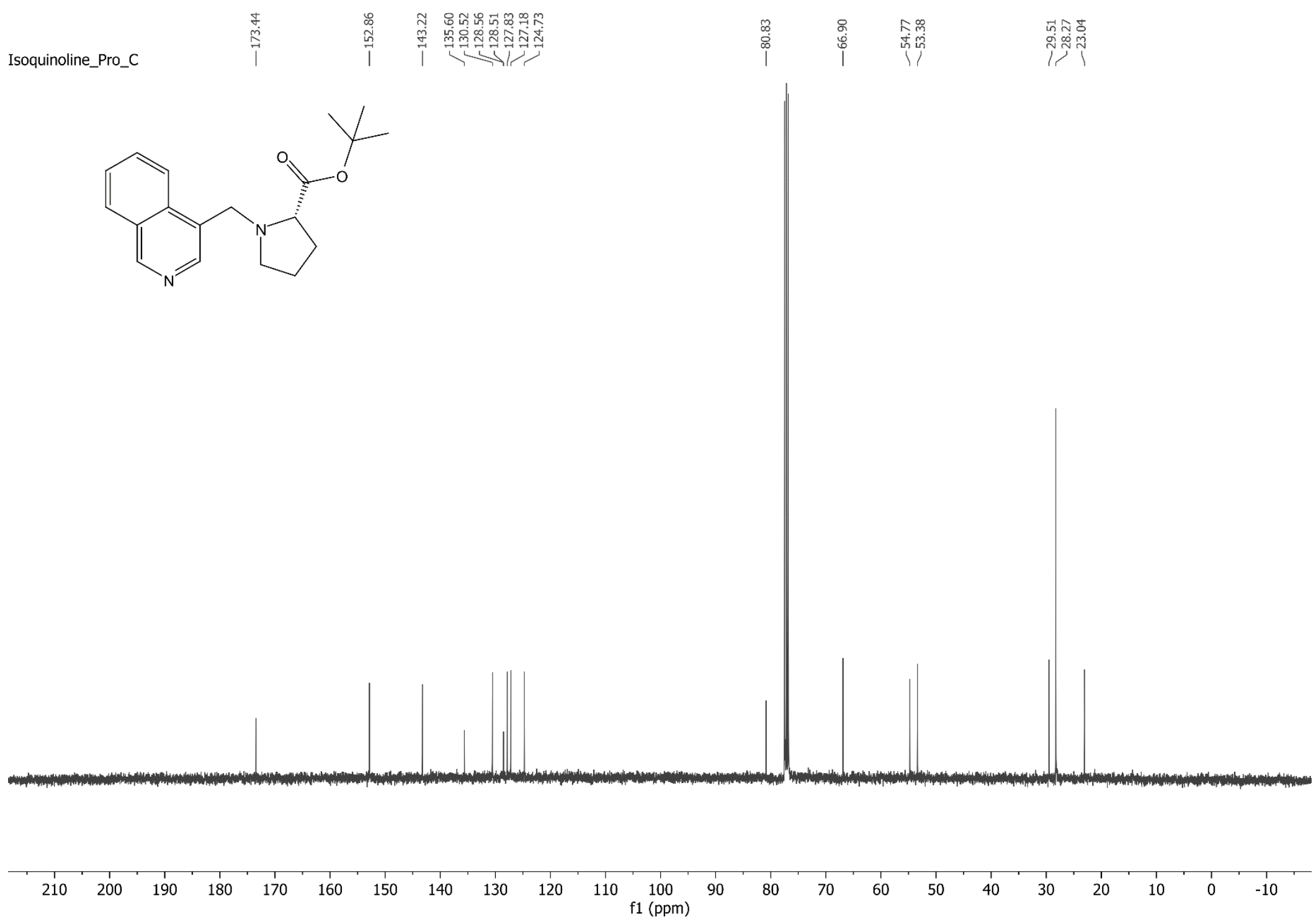

187 
${ }^{1} \mathrm{H}$ NMR $\left(500 \mathrm{MHz}, \mathrm{CDCl}_{3}\right)$ spectrum of $\mathbf{3 p}$

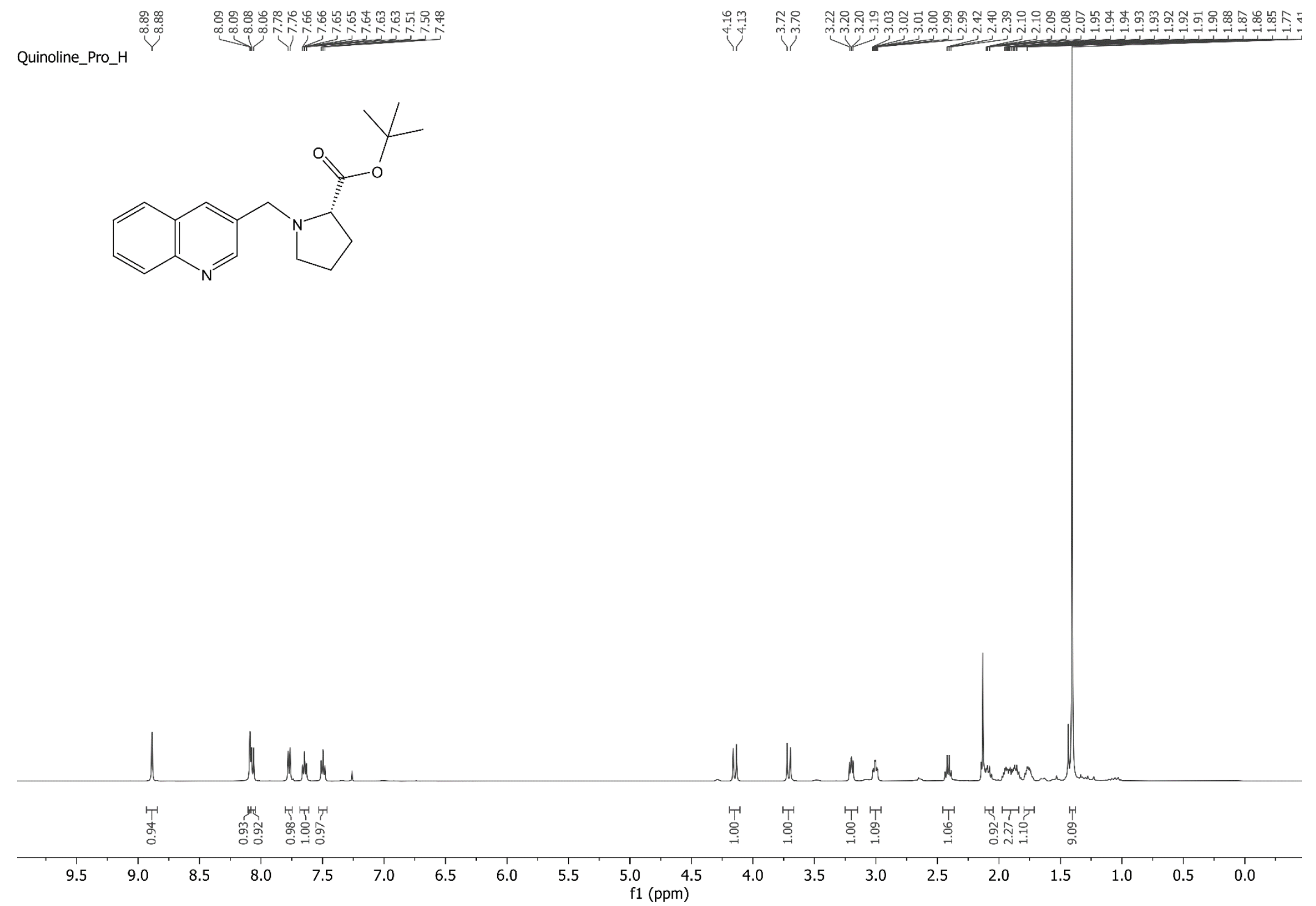


$\left.{ }^{13} \mathrm{C} \mathrm{NMR} \mathrm{(126} \mathrm{MHz,} \mathrm{CDCl}_{3}\right)$ spectrum of $\mathbf{3 p}$
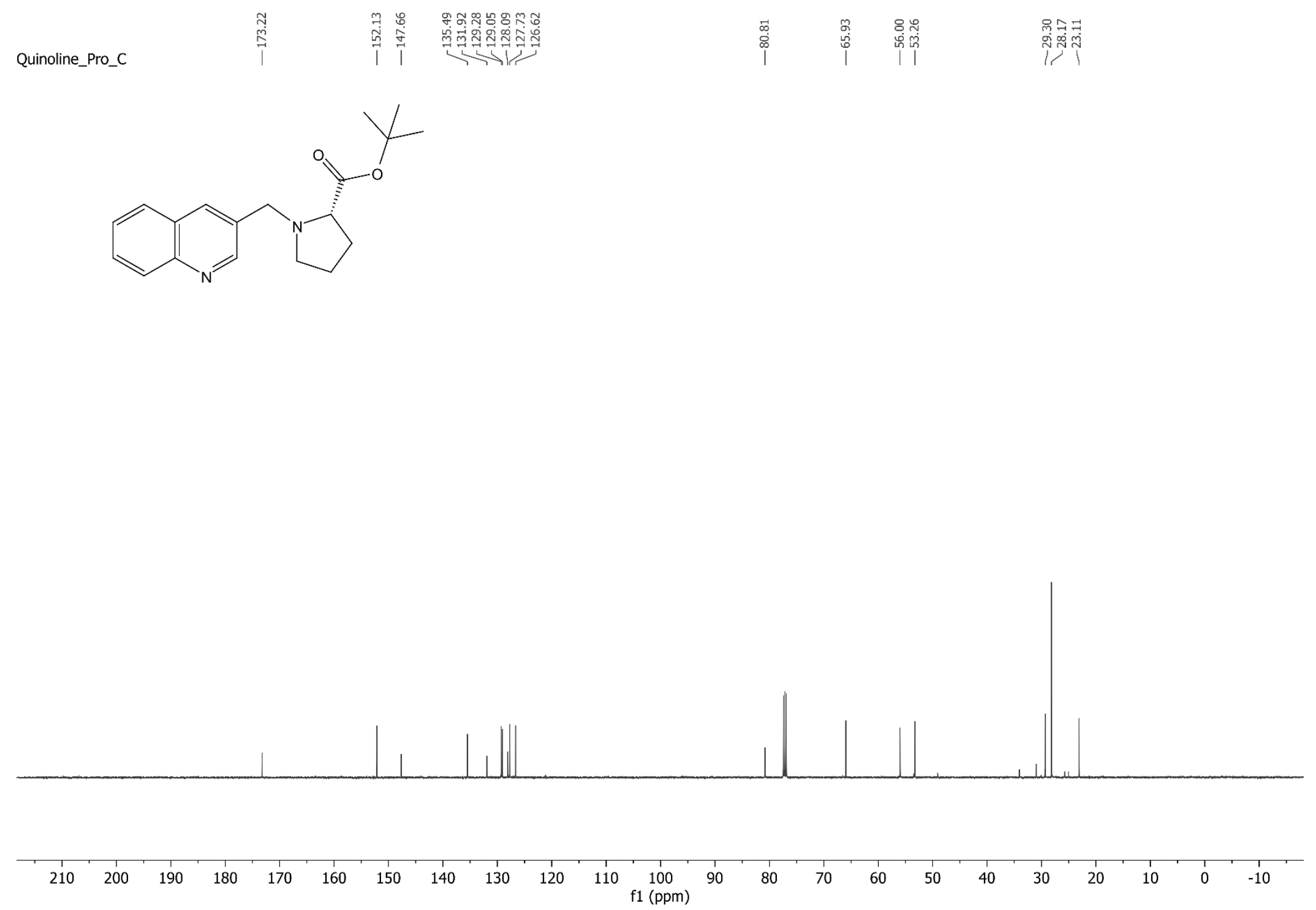
${ }^{1} \mathrm{H}$ NMR (400 MHz, CDCl 3 ) spectrum of $\mathbf{3 q}$

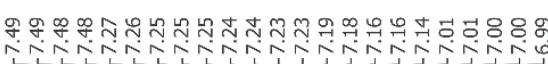

pCN_BnPhe_H

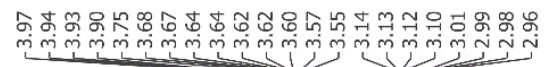

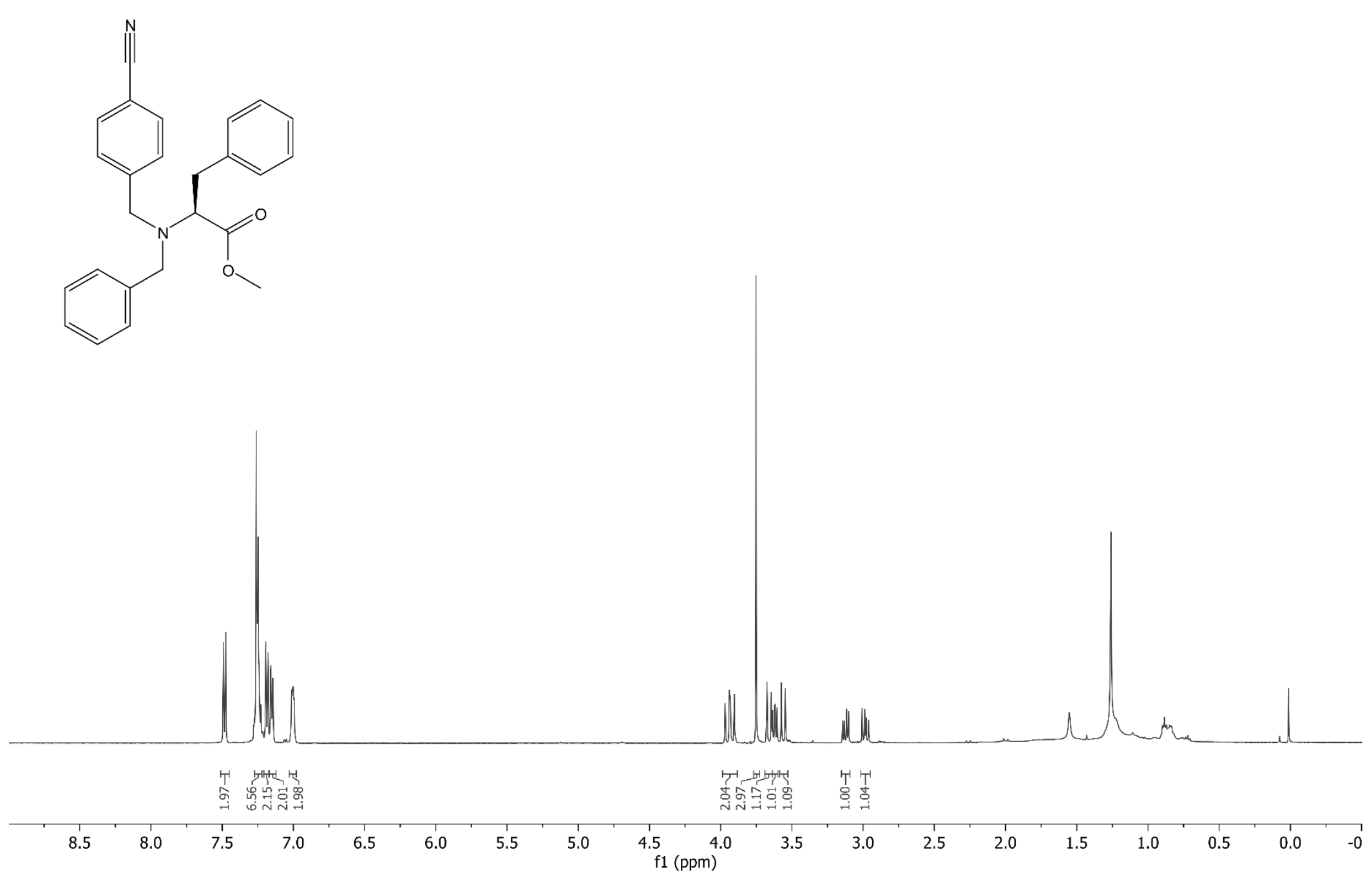




$$
{ }^{2} T
$$


${ }^{1} \mathrm{H}$ NMR $\left(600 \mathrm{MHz}, \mathrm{CDCl}_{3}\right)$ spectrum of $3 \mathbf{r}$
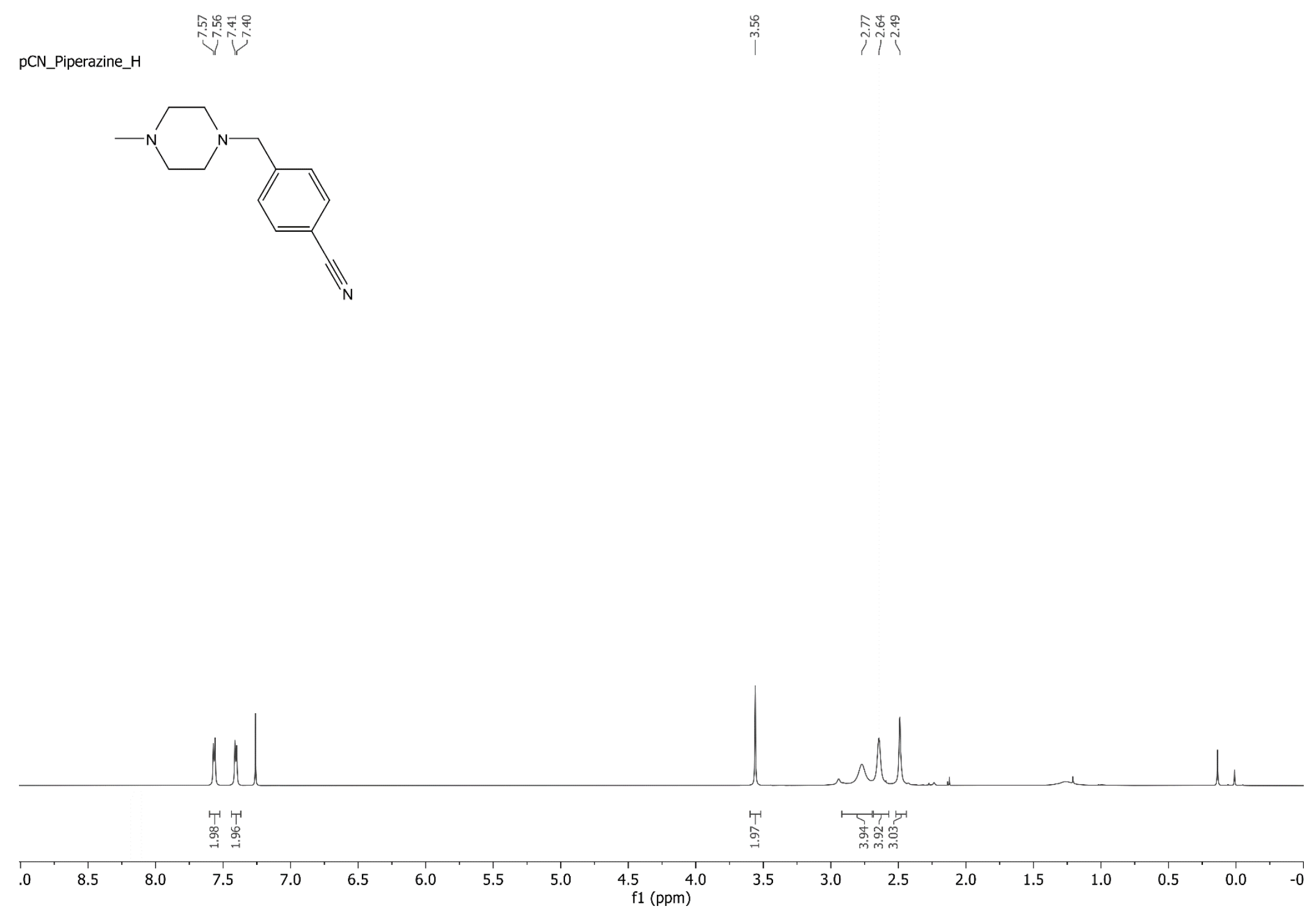
SUPPORTING INFORMATION

${ }^{13} \mathrm{C}$ NMR $\left(151 \mathrm{MHz}, \mathrm{CDCl}_{3}\right)$ spectrum of $\mathbf{3 r}$

pCN_Piperazine_C

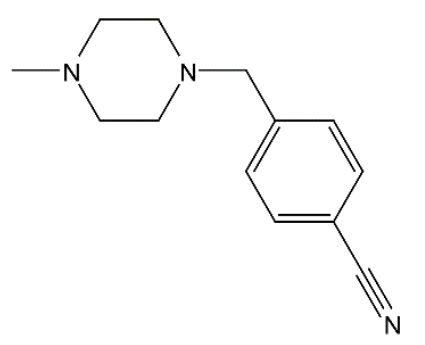

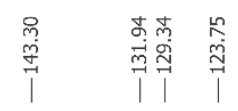

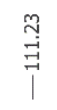

io

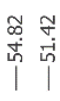

I
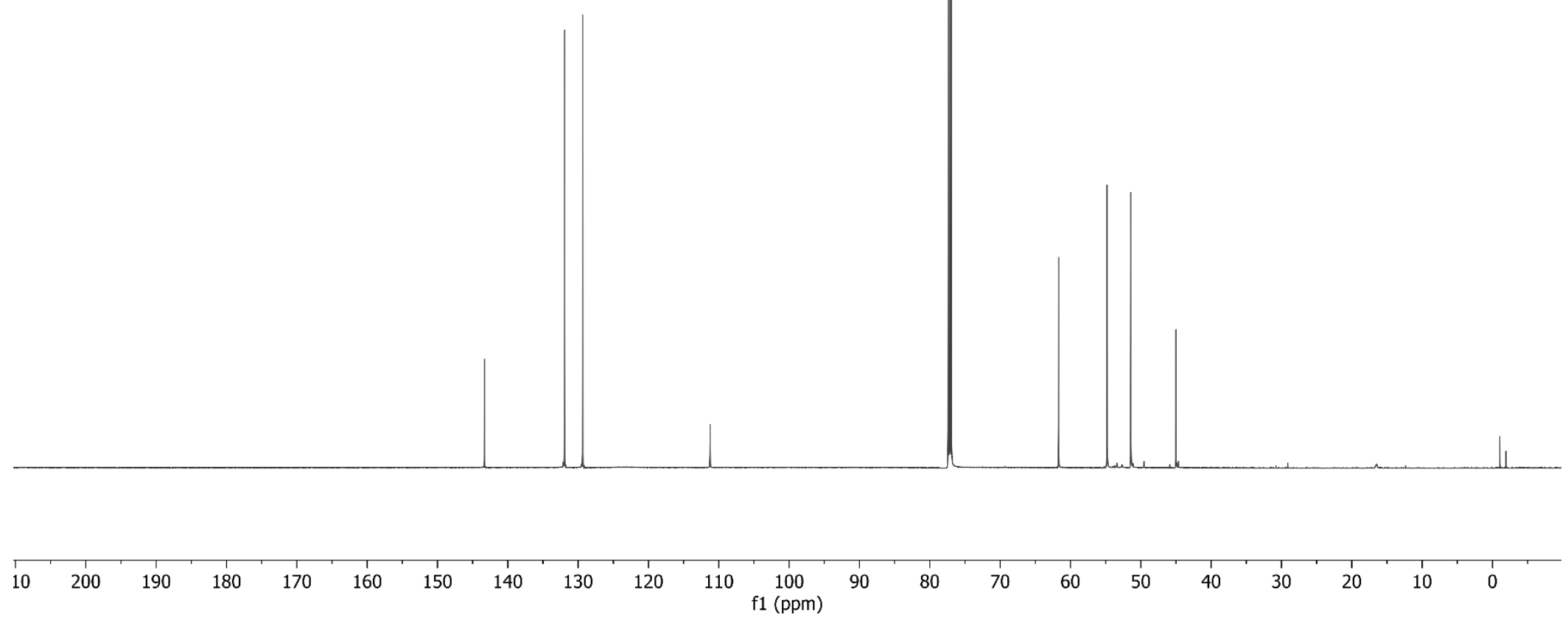

193 
${ }^{1} \mathrm{H}$ NMR $\left(500 \mathrm{MHz}, \mathrm{CDCl}_{3}\right)$ spectrum of $3 \mathbf{s}$

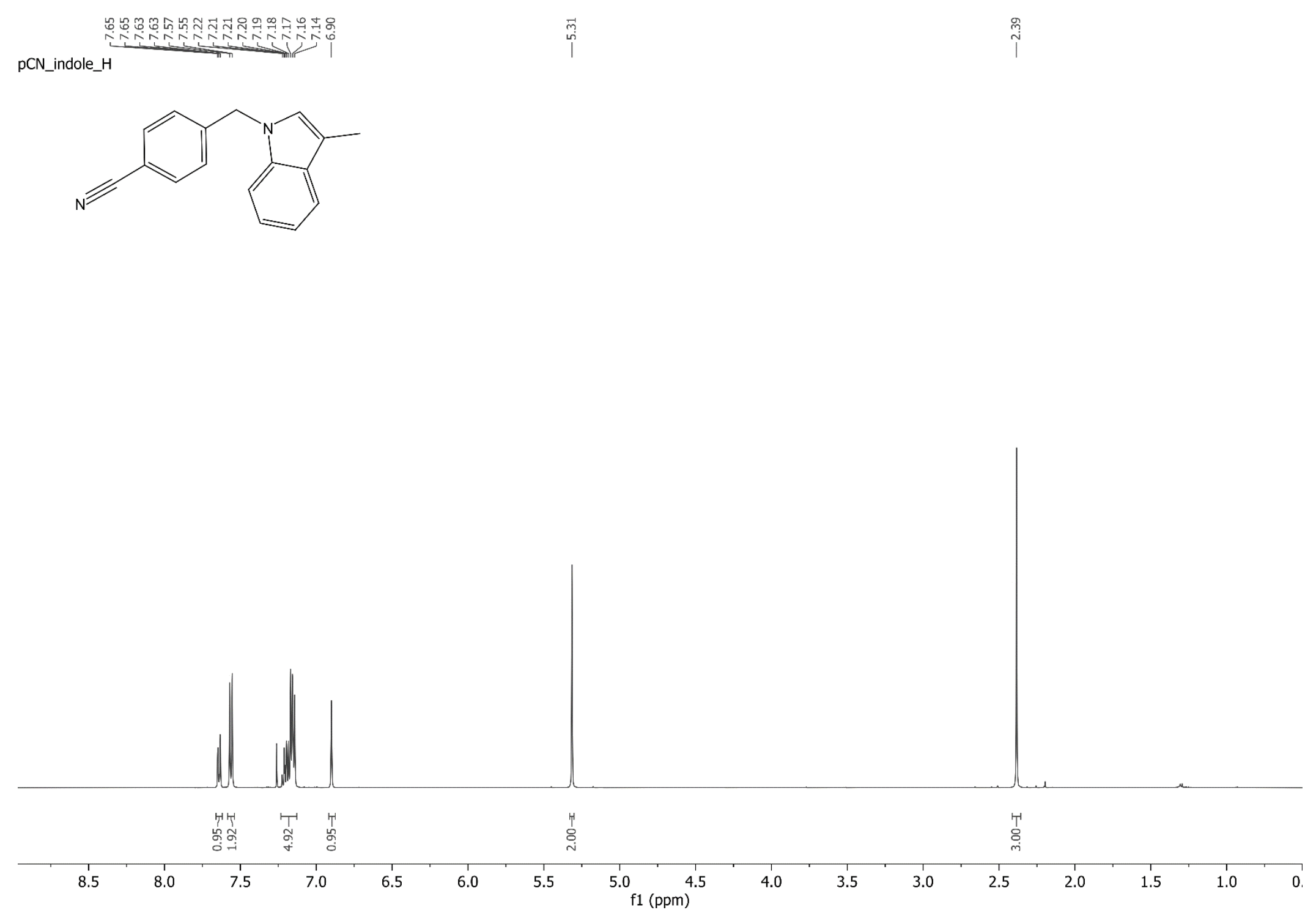


${ }^{13} \mathrm{C}$ NMR $\left(126 \mathrm{MHz}, \mathrm{CDCl}_{3}\right)$ spectrum of $3 \mathbf{s}$
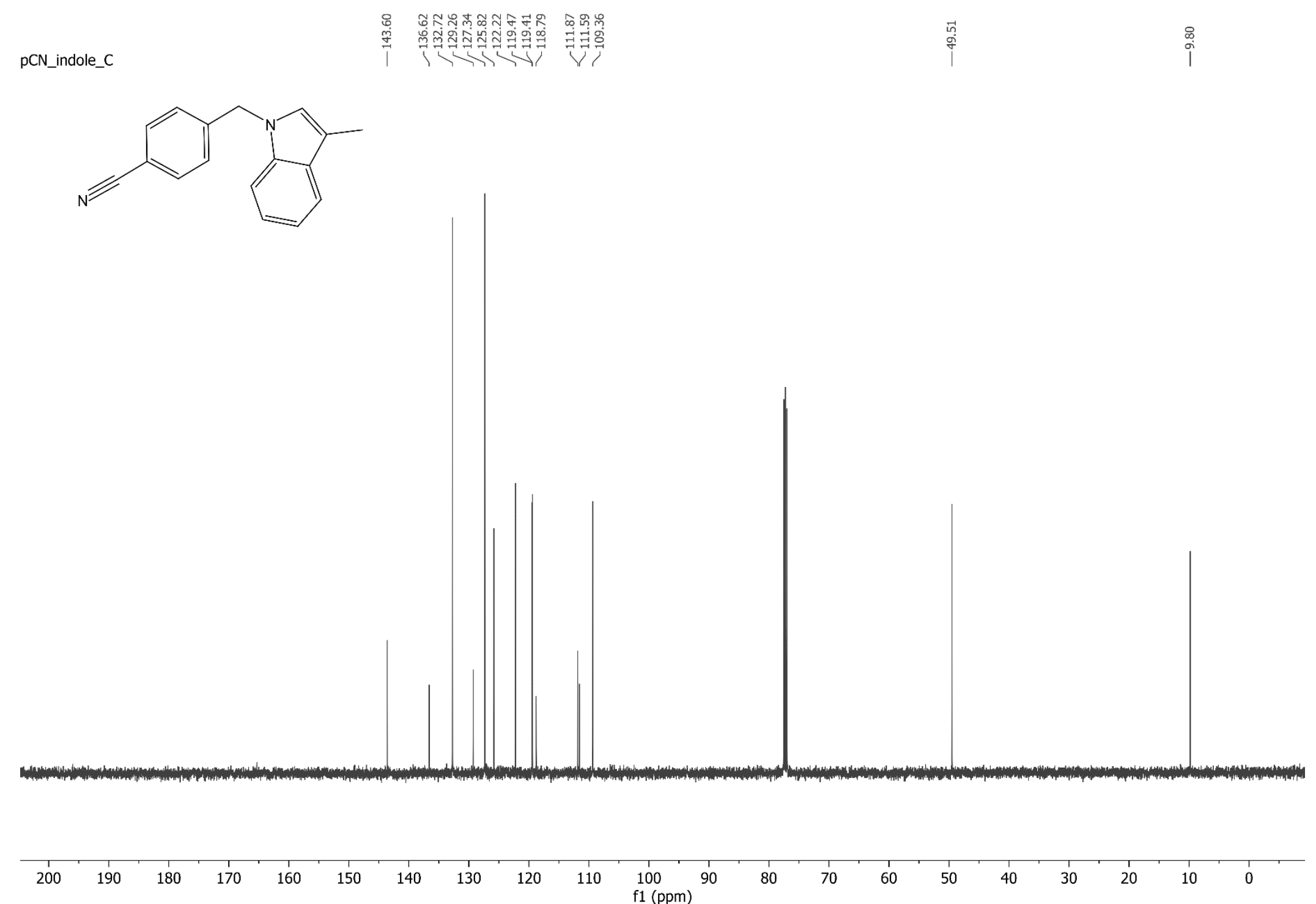
${ }^{1} \mathrm{H} \mathrm{NMR}\left(400 \mathrm{MHz}, \mathrm{CDCl}_{3}\right)$ spectrum of $\mathbf{3 t}$

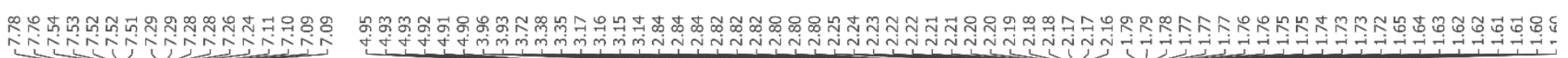
pCN_PhePro_H
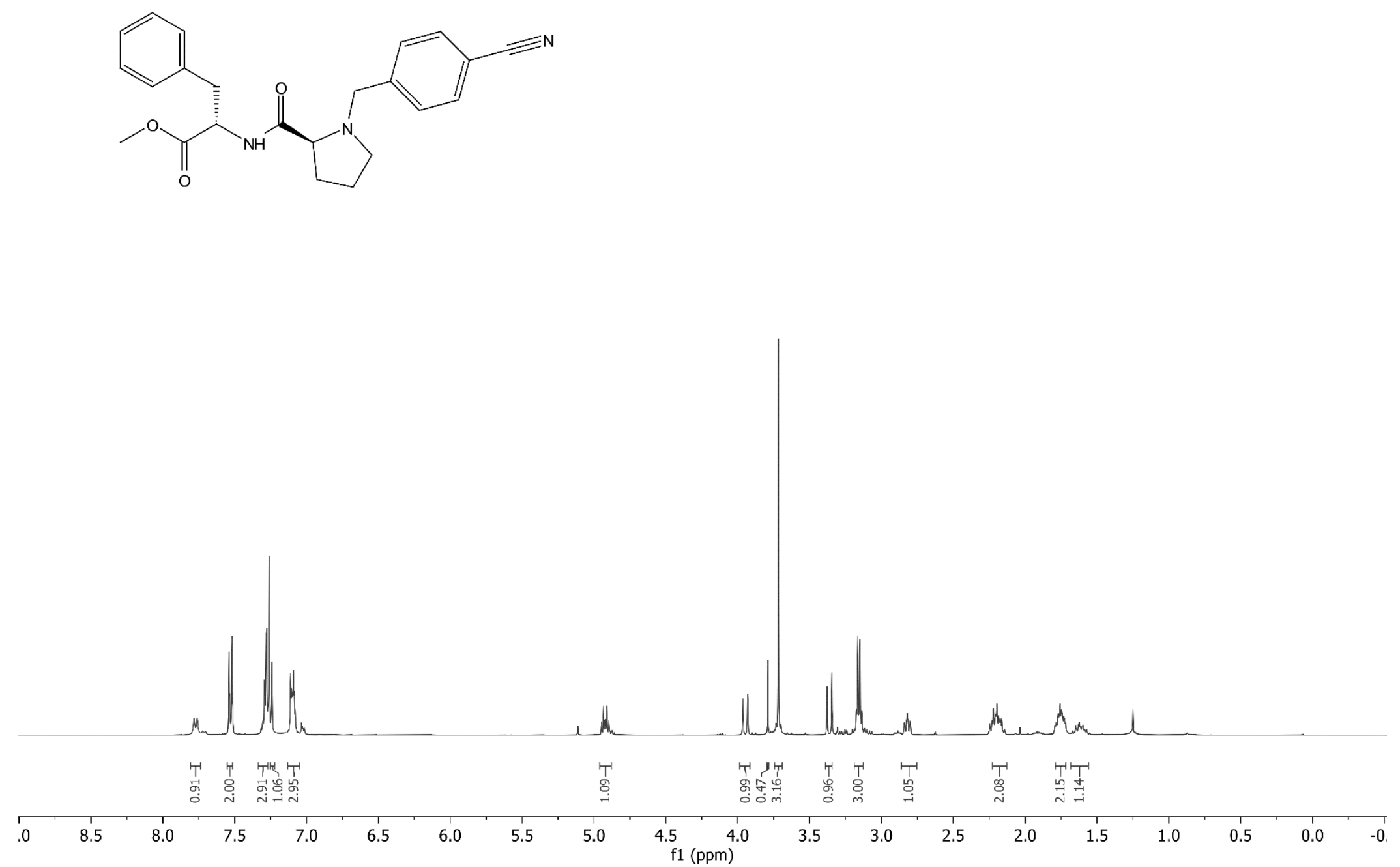
SUPPORTING INFORMATION

${ }^{13} \mathrm{C}$ NMR $\left(101 \mathrm{MHz}, \mathrm{CDCl}_{3}\right)$ spectrum of $\mathbf{3 t}$
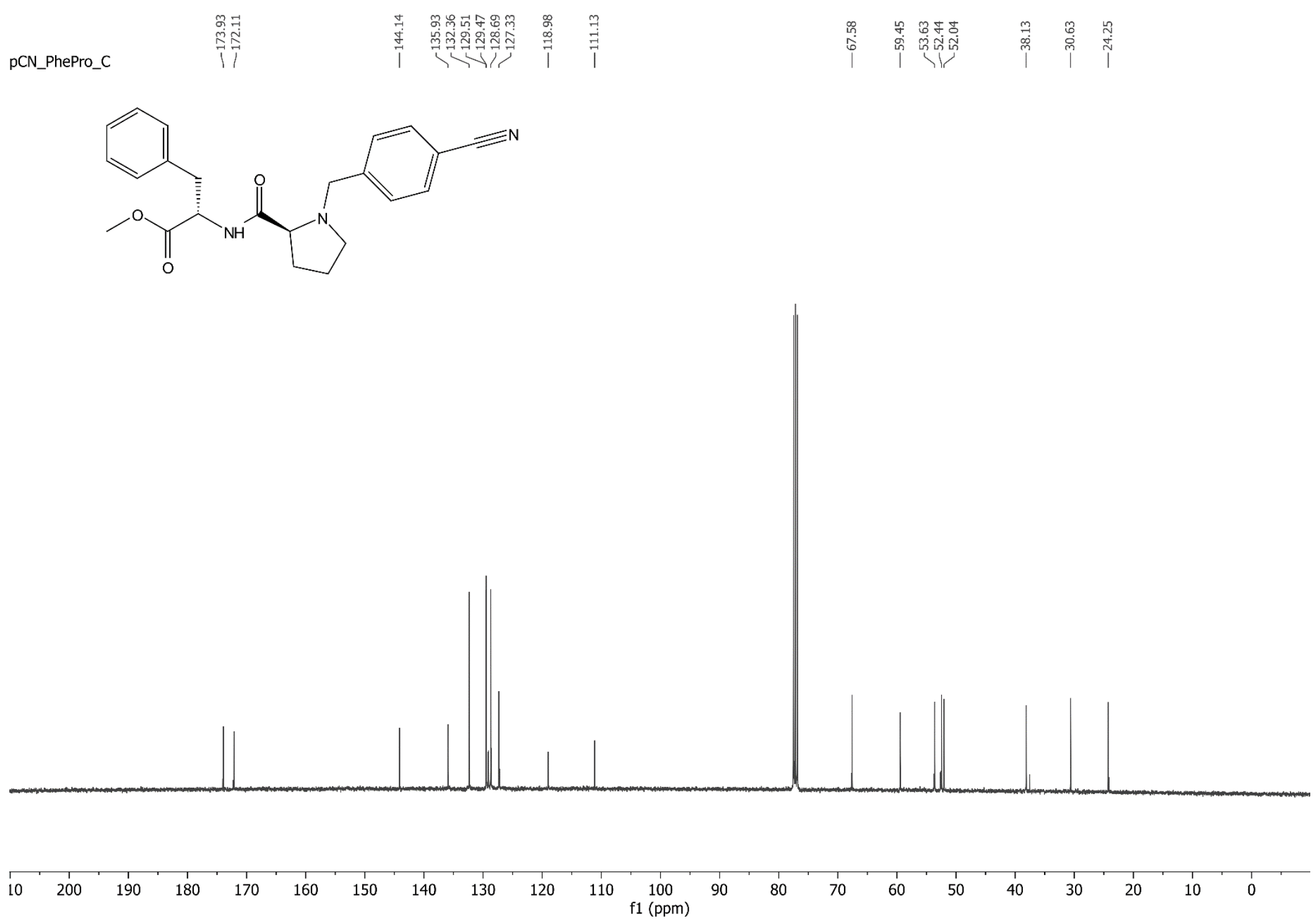

197 
${ }^{1} \mathrm{H}$ NMR $\left(600 \mathrm{MHz}, \mathrm{CDCl}_{3}\right)$ spectrum of 5

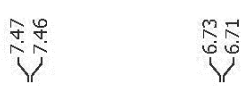

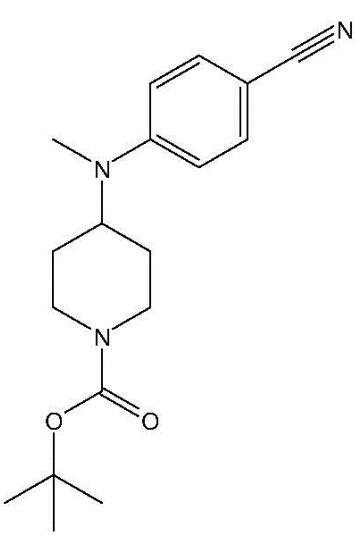

ปิ

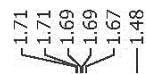

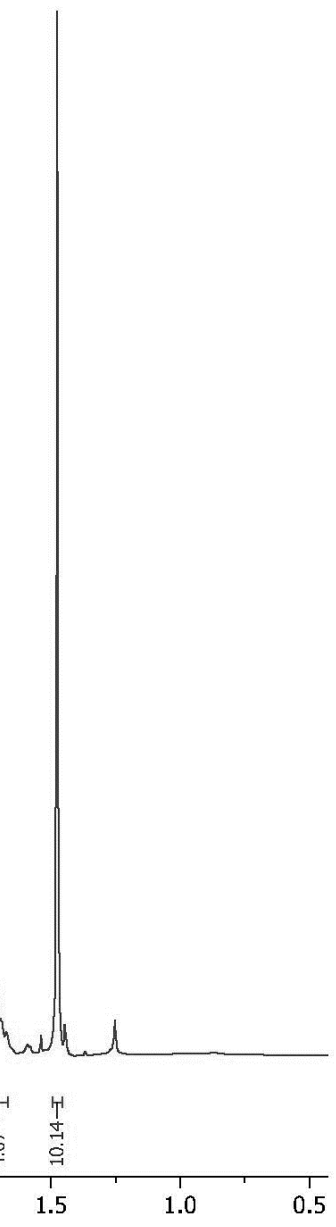


SUPPORTING INFORMATION

${ }^{13} \mathrm{C}$ NMR $\left(151 \mathrm{MHz}, \mathrm{CDCl}_{3}\right)$ spectrum of $\mathbf{5}$

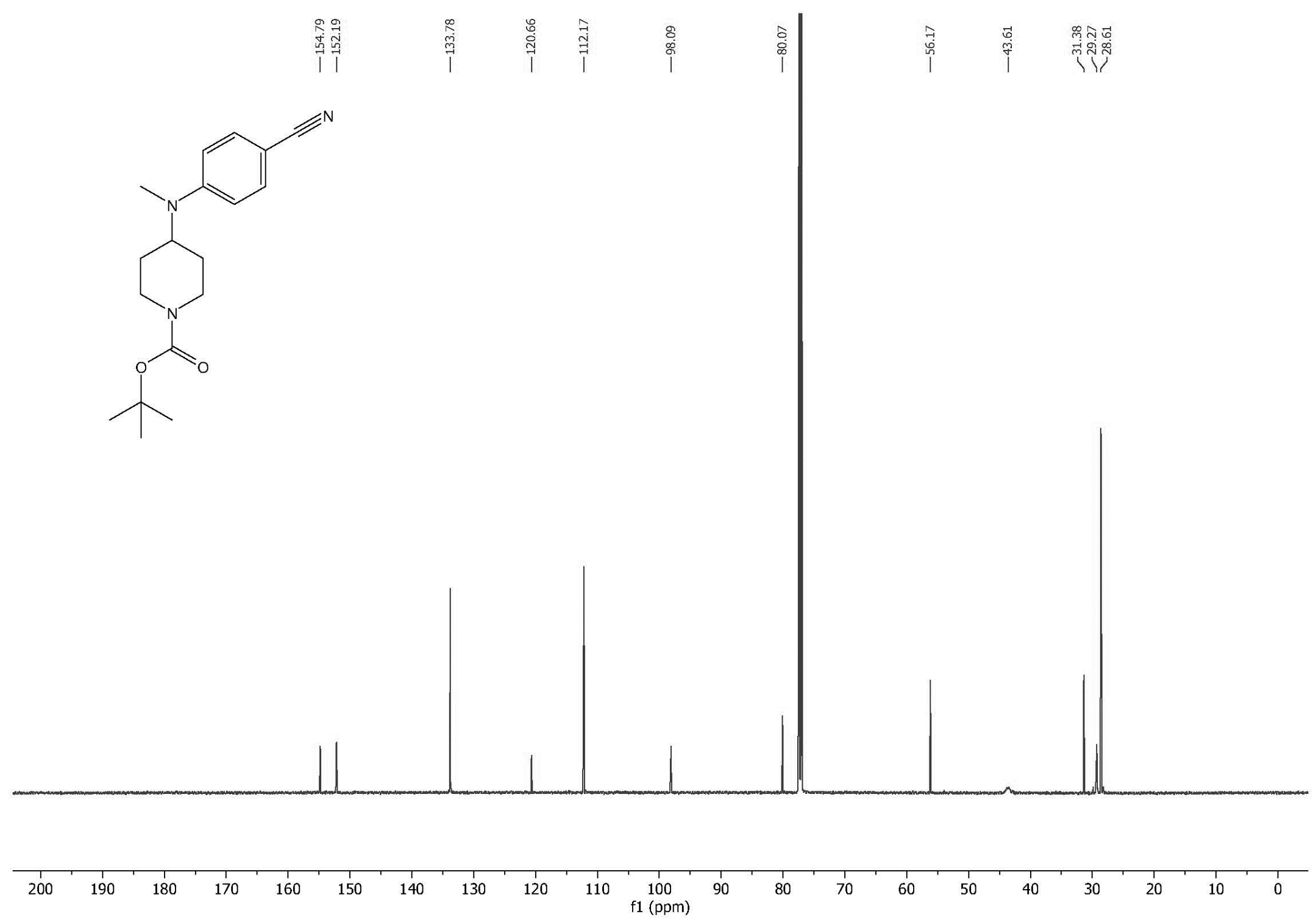

199 
${ }^{1} \mathrm{H}$ NMR $\left(600 \mathrm{MHz}, \mathrm{CDCl}_{3}\right)$ spectrum of 11

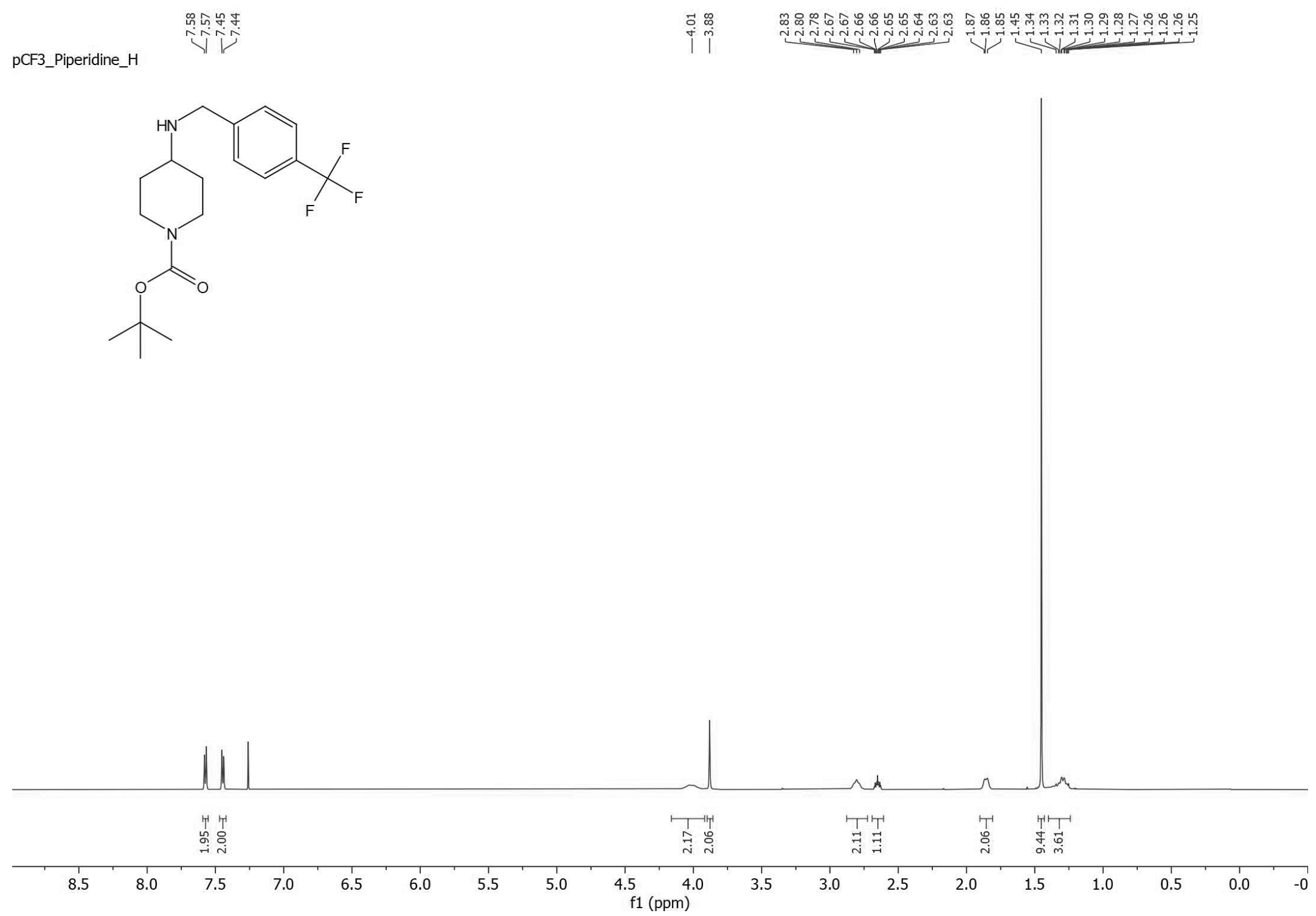


SUPPORTING INFORMATION

${ }^{13} \mathrm{C}$ NMR $\left(151 \mathrm{MHz}, \mathrm{CDCl}_{3}\right)$ spectrum of 11
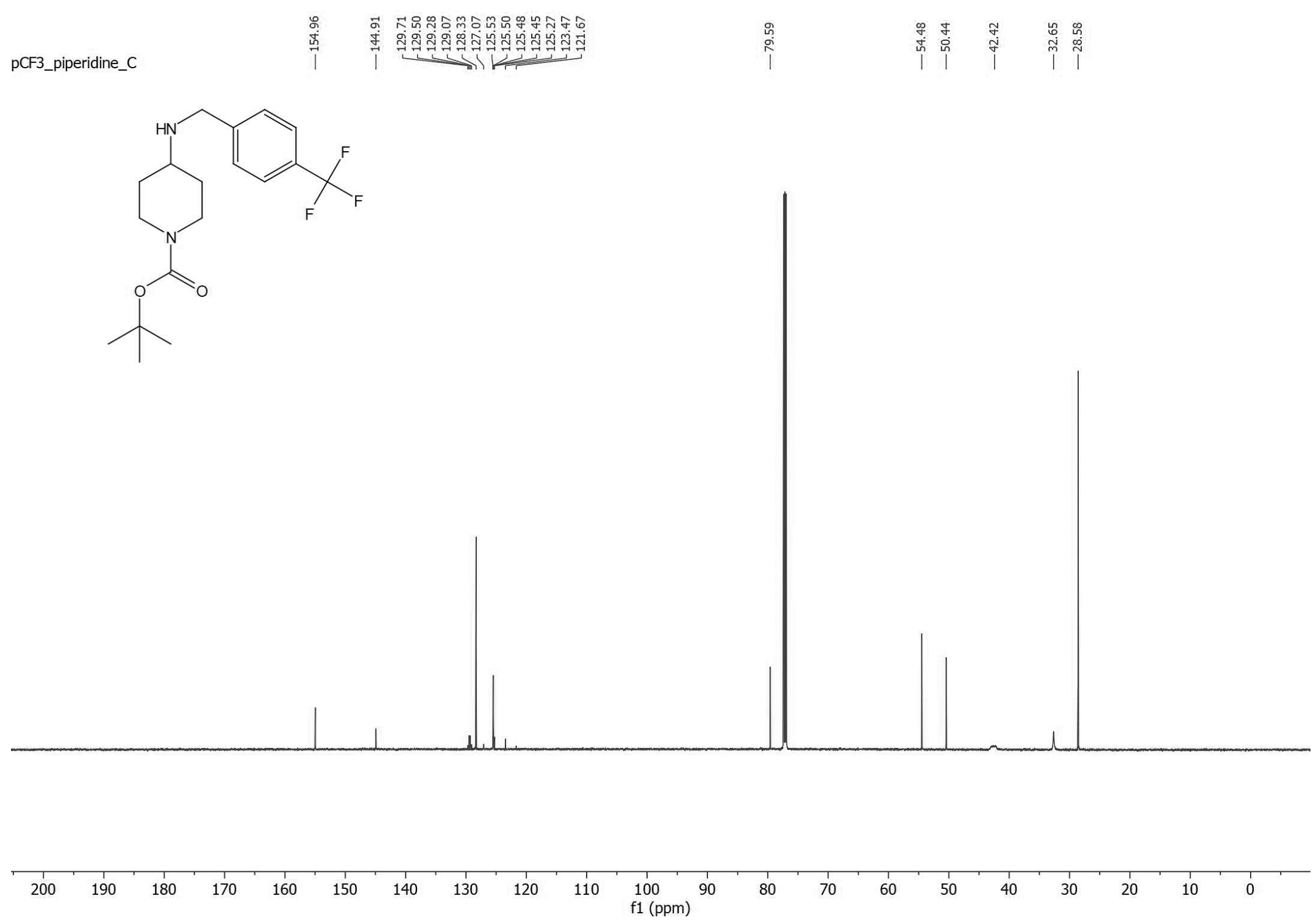

201 
${ }^{19} \mathrm{~F} \mathrm{NMR} \mathrm{(376} \mathrm{MHz,} \mathrm{CDCl}_{3}$ ) spectrum of $\mathbf{5}$

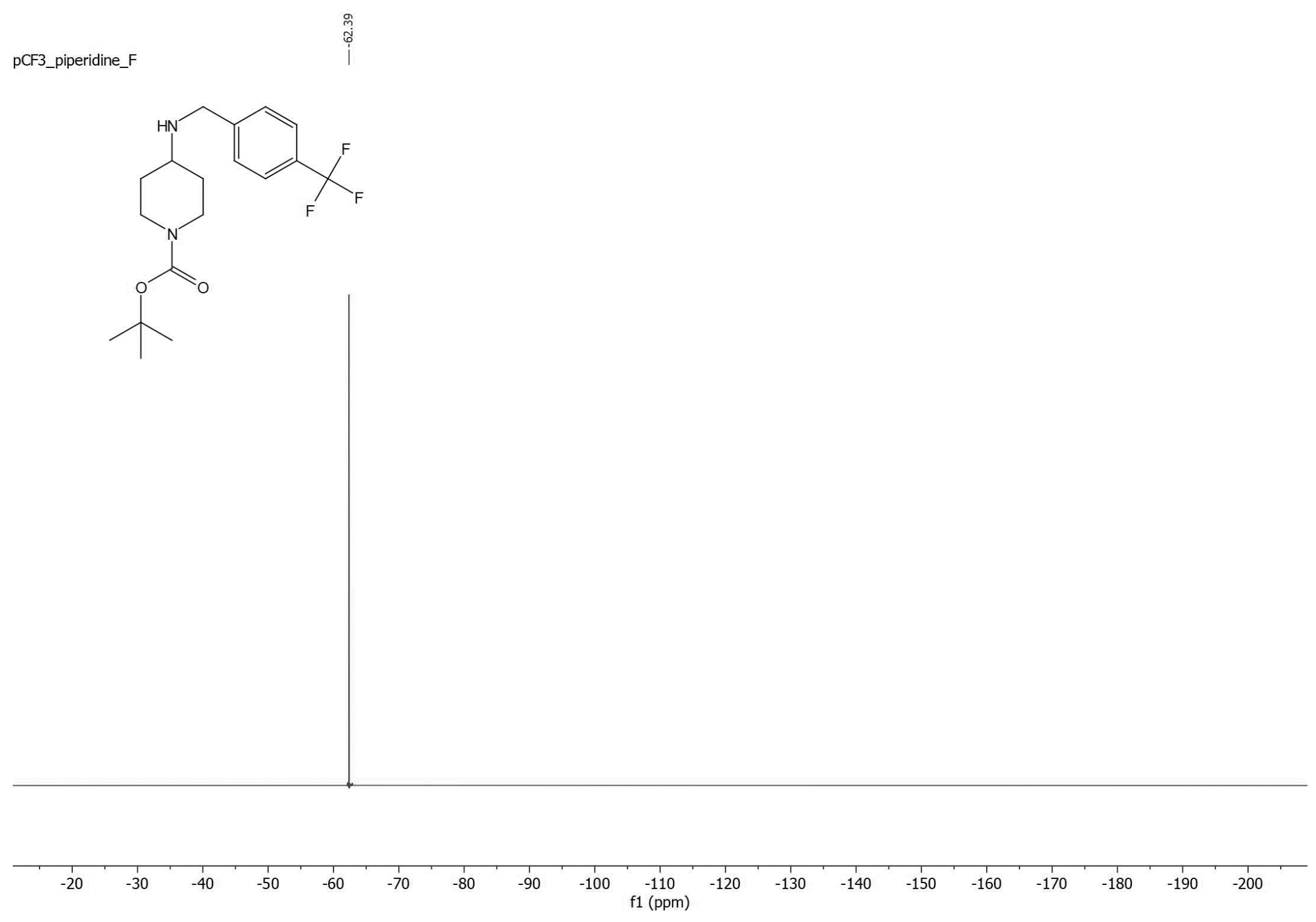

